\title{
Surface-cycling of rhenium and its isotopes
}

by

\author{
Christian Alexander Miller \\ M.Sc., Geology, University of Saskatchewan (2004) \\ B.A., English, University of Saskatchewan (1999) \\ B.Sc., Geology, University of Saskatchewan (1998)
}

Submitted to the Department of Earth, Atmospheric and Planetary

Sciences, MIT \& Department of Marine Chemistry and Geochemistry, WHOI

in partial fulfillment of the requirements for the degree of

Doctor of Philosophy in Chemical Oceanography

at the

\section{MASSACHUSETTS INSTITUTE OF TECHNOLOGY}

and the

\section{WOODS HOLE OCEANOGRAPHIC INSTITUTION}

June 2009

(c) Christian Alexander Miller, MMIX. All rights reserved.

The author hereby grants to MIT and WHOI permission to reproduce and distribute publicly paper and electronic copies of this thesis document in whole or in part.

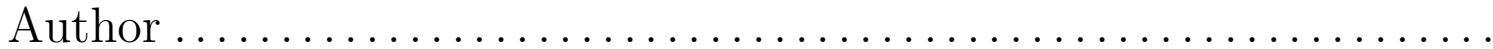

Department of Earth, Atmospheric and Planetary Sciences, MIT \& Department of Marine Chemistry and Geochemistry, WHOI

May 22, 2009

Certified by

Bernhard Peucker-Ehrenbrink Associate Scientist

Thesis Supervisor

Accepted by

Roger Summons

Chair, Joint Committee for Chemical Oceanography 


\title{
Surface-cycling of rhenium and its isotopes
}

\author{
by
}

\author{
Christian Alexander Miller
}

Submitted to the Department of Earth, Atmospheric and Planetary Sciences, MIT

\& Department of Marine Chemistry and Geochemistry, WHOI

on May 22, 2009, in partial fulfillment of the

requirements for the degree of

Doctor of Philosophy in Chemical Oceanography

\section{Abstract}

The application of elemental and isotopic metal palaeoredox tracers to the geologic past rests on an understanding of modern metal cycles. This study reevaluates the surface-cycling of Mo and Re in near-surface reservoirs. Revised river averages of Mo and Re are 1.8- and 7.9-fold larger than previous estimates. The river concentrations

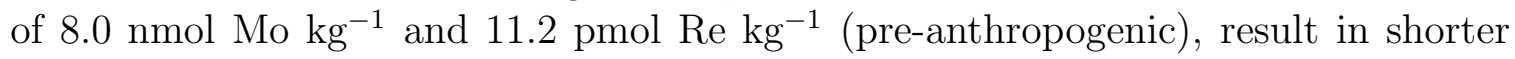
seawater response times of $4.4 \times 10^{5} \mathrm{yr}\left(\tau_{\mathrm{Mo}}\right)$ and $1.3 \times 10^{5} \mathrm{yr}\left(\tau_{\mathrm{Re}}\right.$ pre-anthropogenic). These metals, especially Re, are more sensitive to changing source and sink fluxes than previously thought.

Evaluation of Mo and Re concentrations in high temperature fluids from the Manus Basin indicate that Re is essentially absent from the hydrothermal end member and Mo is present at concentrations considerably lower than ambient seawater. The sink fluxes represented by hydrothermal circulation are negligible in comparison to the revised river source fluxes.

Anthropogenic contributions to the Re flux to seawater are seen in the high concentrations of certain impacted water samples such as those associated with mining sites. It may also be seen in a significant, variable, Re enrichment feature in the Hudson River estuary. This Re enrichment feature is not the result of estuarine mixing or the remobilization of sediment-hosted Re. On the basis of a $\mathrm{Re}-\mathrm{SO}_{4}^{2-}$ correlation we are able to quantify and correct for the anthropogenic Re, which corresponds to $\sim 33 \%$ of the modern river average.

This study documents the development of an analytical method for stable Re isotopes. Though complicated by analyte requirements and ${ }^{187} \mathrm{Re}-{ }^{187} \mathrm{Os}$ decay, Re isotope measurements have a reproducibility of $\pm 0.05 \%$ for analyte concentrations of $20 \mathrm{ng} R e \mathrm{~mL}^{-1}$. Total Re isotopic variability to date is $0.9 \%$. This includes $0.3 \%$ across five commercially available Re products, and $0.5 \%$ across a black shale weathering profile. $\delta^{187} \mathrm{Re}$ variability across the weathering profile was systematic with the most weathered samples showing the most significant ${ }^{187}$ Re depletions. The 
Re isotopic weathering profile is well described by both two-component mixing and Rayleigh fractionation. There are currently insufficient data to discriminate between the two models.

Thesis Supervisor: Bernhard Peucker-Ehrenbrink

Title: Associate Scientist 


\section{Acknowledgements}

Firstly, I must thank my supervisor, Bernhard Peucker-Ehrenbrink, for his guidance and patience over the last $5+$ years. I am proud of the fact that, towards the end, I began to say to myself some of the things he so often said to me. For example,

Hmmmmm ...that's interesting; convince yourself of it!

or,

Hmmmmm ... don't waste your time on that.

I have no doubt that working closely with him has made me a better, more careful, and more thoughtful scientist.

Likewise, I am extremely grateful to Tracy Atwood who kept the Noble Metal Lab running smoothly which, whether I was aware of it or not, facilitated my work in many ways. Thank you Tracy.

Thanks also to the members of my advisory commitee - Ken Sims, Ed Boyle, and Steve Petsch - and to the chair of both my thesis proposal and thesis defenses

- Bill Martin. I greatly appreciate all of the time and effort each of you made.

I owe a great deal to the faculty members of the Department of Marine Chemistry \& Geochemistry (WHOI). In particular, I must thank Dave Glover, Olivier Rouxel, and Jeff Seewald for all of their help with statistics, modeling, programming, mass spectrometry, transition metal isotopes, and ion chromatography. Thanks also to the Chemical Oceanography education co-ordinators: Meg Tivey (for her conscientious and dedicated support) and Mark Kurz (for his relentless encouragement and cheerfulness).

My work would not have been possible without the assistance of past and present members of the WHOI Plasma Mass Spectrometry (ICP) Facility: Lary Ball, Jerzy Blusztajn, Scot Birdwhistell, and Dave Schneider. Thank you all.

Because the Joint Program is more than scientific staff, I must mention the various administrators at both the departmental and institutional level: Vicki McKenna, 
Alla Skorokhod, and Carol Sprague in the EAPS department at MIT. Susan Casso, Sheila Clifford, Donna Mortimer, Mary Murphy, and Mary Zawoysky in the MC\&G department at WHOI. However, special thanks must go to the members of the Academic Programs Office at WHOI, especially Marsha Gomes and Julia Westwater for all of their help ... with everything.

Because there is more to life than just research, I must thank the many classmates and friends both here and at home. I count myself extremely fortunate that there are too many to be listed here though I must single out Peter Canovas, Dreux Chappel, Paul Craddock, Francesca DeLeonardis, and Desirée Plata - the Chemistry cohort who made the journey with me - as well as Erin Banning, Bridget Bergquist, Joanna Gyory, Mike Jakuba, Christina Maki, Travis Poole, and Eoghan Reeves the roommates who put up with me. I must also thank Patty Thille, my best friend.

Finally, I must thank the members of my family: my brother Andrew and sisterin-law Jen, and especially my parents Jim and Mary Miller to whom this thesis is dedicated. 


\section{Contents}

1 Introduction $\quad 15$

1.1 Rationale and thesis structure $\ldots \ldots \ldots \ldots \ldots$

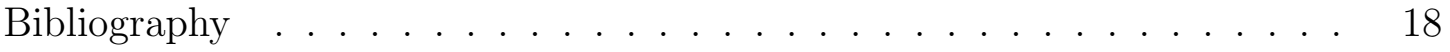

2 A reassessment of molybdenum and rhenium surface cycling $\quad 21$

2.1 Introduction . . . . . . . . . . . . . . . . . 21

2.2 Background: surface cycling of Mo and Re . . . . . . . . . . 22

2.3 Materials and Methods . . . . . . . . . . . . . . . . . . . . . 24

2.3 .1 Sample collection . . . . . . . . . . . . . . . . 24

2.3.2 Mo and Re concentrations . . . . . . . . . . . . . . 26

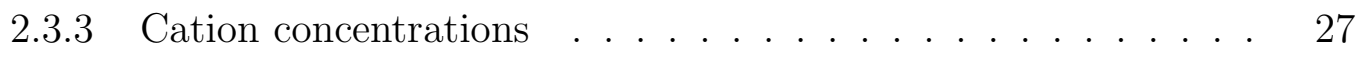

2.3.4 Anion concentrations . . . . . . . . . . . . . . . . . . . . . . 29

2.4 Results . . . . . . . . . . . . . . . . . . . . 30

2.5 Discussion . . . . . . . . . . . . . . . . . . . . . 60

2.5.1 Complexities in determining "global" river characteristics . . . 60

2.5.2 Calculation of present-day average Mo and Re concentrations in rivers . . . . . . . . . . . . . . . . . . 65

2.5.3 Anthropogenic contributions and the pre-anthropogenic world river average of $\operatorname{Re} \ldots \ldots \ldots \ldots . \ldots \ldots$

2.5.4 Continental sources of Mo and Re . . . . . . . . . . 74

2.5.5 Estuarine behavior of Mo and Re . . . . . . . . . 79 
2.5.6 Mo and Re in high temperature hydrothermal fluids from the Manus Basin . . . . . . . . . . . . . . . 87

2.5.7 Response times and modeling of Mo and Re inventories in seawater . . . . . . . . . . . . . . . . 90

2.6 Conclusions . . . . . . . . . . . . . . . . . . . . . 95

2.7 Acknowledgements . . . . . . . . . . . . . . . . . . . 96

2.A Modeling seawater inventories of Mo and Re . . . . . . . . 98

Bibliography . . . . . . . . . . . . . . . . . . . . 105

3 Precise determination of rhenium isotopic composition by multicollector inductively-coupled plasma mass spectrometry 125

3.1 Introduction and previous work . . . . . . . . . . . 126

3.2 Analytical methods . . . . . . . . . . . . . . . . . . . . . . . 129

3.2 .1 Materials . . . . . . . . . . . . . . . . . . . 129

3.2 .2 Sample processing . . . . . . . . . . . . . . . . . 131

3.2 .3 Mass spectrometry . . . . . . . . . . . . . . . . . . 131

3.3 Correction of instrumental mass fractionation . . . . . . . . . 132

3.3.1 Standard-bracketing correction . . . . . . . . . . . . 132

3.3 .2 External correction . . . . . . . . . . . . . . . . 133

3.4 Rhenium isotopic variability in commercially available materials . . . 148

3.5 Concerns regarding natural samples . . . . . . . . . . . . . . . . . . 149

3.5.1 Analyte requirements . . . . . . . . . . . . . . . . . 149

3.5.2 Chromatographic fractionation . . . . . . . . 150

3.5.3 Matrix effects . . . . . . . . . . . . . . . . . 153

3.5.4 Decay to Os and sparging . . . . . . . . . . . . . . 153

3.6 Conclusions . . . . . . . . . . . . . . . . . . . . . . . . . . 157

3.7 Acknowledgments . . . . . . . . . . . . . . . . . . . . 158

3.A Mass fractionation in the $\mathrm{W}$ system . . . . . . . . . . . . . 159

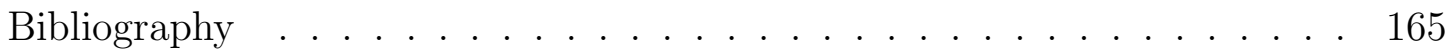


4 Rhenium isotopic variation across a black shale weathering profile 175

4.1 Introduction . . . . . . . . . . . . . . . . . . . 175

4.2 Materials and methods . . . . . . . . . . . . . 176

4.2.1 Sampling ... . . . . . . . . . . . . . . 176

4.2 .2 Elemental analyses . . . . . . . . . . . . . . . . 177

$4.2 .3 \quad \delta^{187}$ Re analyses . . . . . . . . . . . . . . . . . 178

4.3 Results and Discussion . . . . . . . . . . . . . . . . . 179

4.3.1 Elemental variability . . . . . . . . . . . . . . . . . 179

4.3.2 Re isotopic variability . . . . . . . . . . . . . . 181

4.3.3 Testing models of Re fractionation . . . . . . . . . . . . . 188

4.4 Conclusions . . . . . . . . . . . . . . . . . . . 190

4.5 Acknowledgements . . . . . . . . . . . . . . . . . . 190

Bibliography ............................. 192

5 Conclusions and future avenues of research 197

Bibliography ........................... 201 


\section{List of Figures}

2-1 Map of sample locations . . . . . . . . . . . . . 25

2-2 Number of rivers vs. cumulative $\mathrm{H}_{2} \mathrm{O}$ flux for world rivers . . . . . . 61

2-3 Water flux and Mo concentrations for the Yenisei River, 2004 . . . . 63

2-4 Water fluxes of the Mississippi River and its major tributaries . . . . 64

2-5 Proportion of daily calculated Re concentration vs. daily calculated Re flux for each of the major Mississippi tributaries . . . . . . . 66

2-6 Molybdenum and $\mathrm{SO}_{4}^{2-}$ in surface waters, crustal rocks, $\mathrm{MoS}_{2}$, and

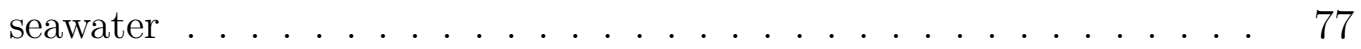

2-7 Rhenium and $\mathrm{SO}_{4}^{2-}$ in surface waters, crustal rocks, sulfides, and seawater 78

2-8 Molybdenum and Re concentrations vs. salinity, Mississippi River es-

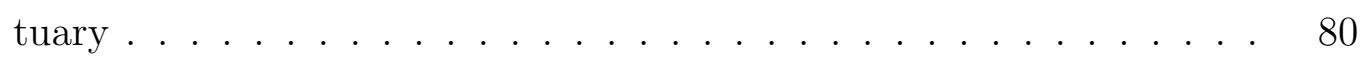

2-9 Molybdenum concentration vs. salinity, Hudson River estuary A-D . 81

2-9 Rhenium concentration vs. salinity, Hudson River estuary E-H . . . . 82

2-10 Rhenium concentration vs. latitude, Hudson River estuary A-C . . 84

2-10 Rhenium concentration vs. latitude, Hudson River estuary D-F . . 85

2-11 Results of modeling Mo and Re seawater inventories . . . . . . . . 94

2-12 Scenarios used for Mo and Re seawater inventory modeling . . . . . . 104

3-1 Re ionization efficiency vs. temperature for PTIMS and plasma ion

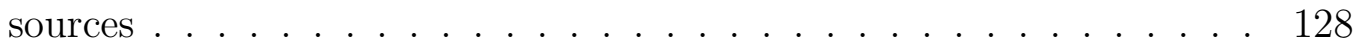

3-2 Ion beam intensity and within-run uncertainty vs. solution Re concentration . . . . . . . . . . . . . . . . . . 130 
3-3 Theoretical plot of instrumental mass fractionation in $\log _{e}$ space . . . 137

3-4 Plot of of ${ }^{187} \mathrm{Re} /{ }^{185} \mathrm{Re}$ vs. ${ }^{186} \mathrm{~W} /{ }^{184} \mathrm{~W}$ for mixed standard solutions of SRM 989 and SRM 3163 analyses in $\log _{e}$ space . . . . . . . . . . . . . 139

3-4 Caption ............................. 140

3-5 Abundance sensitivity and W- and Re-hydride formation . . . . . . . 147

3-6 Analyte requirements for Re isotopic analysis . . . . . . . . . . . . . 151

3-7 Re isotope effects of column chromatography . . . . . . . . . . . . . . 152

3-8 Mass scans of actual and artificial black shale matrices . . . . . . . . 154

3-8 Residual Os, ${ }^{187} \mathrm{Re} /{ }^{185} \mathrm{Re},{ }^{186} \mathrm{~W} /{ }^{184} \mathrm{~W}$, and ${ }^{192} \mathrm{Os} /{ }^{190}$ Os data from a 5 hour sparging experiment . . . . . . . . . . . . . . 156

3-8 Caption . . . . . . . . . . . . . . . . . . 157

3-9 A-C: Three-isotope plots of ${ }^{183} \mathrm{~W} /{ }^{184} \mathrm{~W}$ vs. ${ }^{186} \mathrm{~W} /{ }^{184} \mathrm{~W}$. . . . . . . . 160

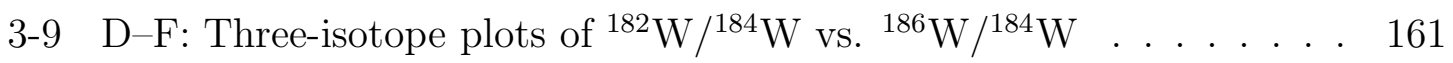

3-9 Caption . . . . . . . . . . . . . . . . . . . . 162

4-1 New Albany Shale weathering profile, Clay City, KY, USA . . . . . . 177

4-2 Elemental variation across the New Albany Shale weathering profile . 180

$4-2$ Caption ............................... 181

4-3 Re isotopic composition and model results for NAS weathering profile 182

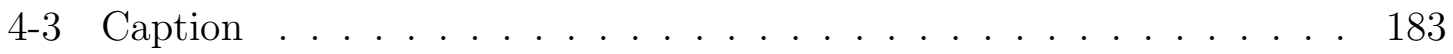

4-4 Re-Os modeling of NAS weathering profile . . . . . . . . . . 185

$4-4$ Caption ......................... 186

4-5 Metal solvent-extraction from black shale . . . . . . . . . . . . 189 


\section{List of Tables}

2.1 A: Listing, sampling dates, and $\mathrm{H}_{2} \mathrm{O}$ fluxes for Arctic river and Mississippi tributaries time-series . . . . . . . . . . . . . . . . 34

2.1 B: Chemical concentration data for Arctic river and Mississippi tributaries time-series $\ldots \ldots \ldots \ldots \ldots \ldots$

2.2 A: Listing, basin characteristics and sampling locations for exorheic rivers analyzed in this study . . . . . . . . . . . . . . 39

2.2 B: Chemical concentrations of exorheic basins analyzed in this study . 40

2.3 A: Listing, sampling date, and location information for tributary and exorheic river samples . . . . . . . . . . . . . . . 45

2.3 B: Listing and chemical data for tributary and exorheic river samples. $\quad 50$

2.4 A: Locations of precipitation and mine water samples . . . . . . . 51

2.4 B: Chemical data for precipitation and mine water samples . . . . . 52

2.5 Listing, locations, dates and chemical data for one Mississippi estuary profile and six Hudson estuary profiles . . . . . . . . . . 58

2.6 Re and Mo in hydrothermal fluids from the Manus Basin . . . . . 59

2.7 Chemical concentration summaries for Graham drainage regions . . . 68

2.8 Comparison of world river average chemical concentrations . . . . . 69

2.9 Ionic radii of Mo and $\mathrm{Re} \ldots \ldots \ldots \ldots \ldots \ldots$

3.1 Observed and calculated fractionation slopes for W-correction of Re . 141

$3.2 \quad \delta^{187}$ Re values and uncertainties of mixed SRM 989 (Re) and SRM 3163 (W) solutions . . . . . . . . . . . . . . . . . . . . . 144 
3.3 $\quad \delta^{187} \mathrm{Re}$ values and uncertainties for commercially available Re materials 149

3.4 Observed and theoretically-calculated fractionation trends for various correcting and corrected $\mathrm{W}$ isotope ratios . . . . . . . . . . 164

4.1 Element concentration and $\delta^{187}$ Re data from the NAS weathering profile, near Clay City, KY . . . . . . . . . . . . . . . . . . . . . . . 179 


\section{Chapter 1}

\section{Introduction}

Marine environments such as the Black Sea serve as modern analogues to depositional settings that produced the extensive black shales of the Palaeozoic and Mesozoic (RAvizza et AL., 1991; Morford And Emerson, 1999). Black shales represent the organic-rich end member of the sedimentary organic carbon $\left(\mathrm{C}_{\text {org }}\right)$ reservoir. Along with atmospheric weathering of $\mathrm{Ca}$ - and $\mathrm{Mg}$ - silicates, $\mathrm{C}_{\text {org }}$ burial serves as a sink for atmospheric $\mathrm{CO}_{2}$ (URey, 1952; Walker ET AL., 1981; Berner et AL., 1983; BERNER, 1992).

The high $\mathrm{C}_{\text {org }}$ contents of modern and ancient anoxic sediments foster the accumulation of a suite of redox-sensitive trace metals, of which Mo and Re show the greatest degrees of enrichment (Bertine and Turekian, 1973; Ravizza and Turekian, 1989; Emerson And Huested, 1991; Ravizza et Al., 1991; Crusius et Al., 1996; Morford And Emerson, 1999). It has been proposed that the $0.3 \%$ of the seafloor overlain by modern anoxic waters account for up to $\sim 30-43 \%$ of the total sink for these metals, and that more widespread ocean anoxia in the geologic past resulted in a decrease in the seawater inventory of these metals (EMERson and Huested, 1991; Colodner et Al., 1993; Morford and Emerson, 1999; Siebert et Al., 2003; Algeo, 2004).

Studies have used Mo to describe local and global redox conditions in increasingly 
quantitative fashion (Siebert ET AL., 2003; Arnold Et Al., 2004; Algeo And LyOns, 2006). The $\delta^{98 / 95}$ Mo signatures of ancient organic-rich sediments are used to determine the global redox conditions at the time of deposition (SIEBERT ET AL., 2003; Arnold et Al., 2004; Poulson et Al., 2006; Pearce et Al., 2008; NeuBERT ET AL., 2008). More recently, stable isotope fractionation of $U$ has also been investigated (WEYER ET AL., 2008), though to date, the stable isotope fractionation of Re has not.

The quantitative application of, for example, Mo to the palaeoenvironment depends on our understanding of present-day Mo cycling in near-surface reservoirs. As these elemental and isotopic proxies are increasingly being applied to the geologic past, it becomes more important that these modern cycles are properly understood.

\subsection{Rationale and thesis structure}

Modern fluxes of reductively-enriched metals from seawater are difficult to quantify (Morford AND EMERSOn, 1999) so modern response times are based on studies of fluxes to seawater. For Mo and Re, this is mainly thought to be the dissolved river flux (Bertine And Turekian, 1973; Colodner et Al., 1993). We have selected Mo and Re as they are the most widely used isotope palaeoredox indicator, and the most enriched under reducing sedimentary environments, respectively.

Present estimates of the world river average concentrations are based on consideration of three (Mo) and four (Re) rivers (Bertine And Turekian, 1973; Colodner ET AL., 1993). Consideration of such small sample sets renders any kind of regional averaging meaningless because so little of the global drainage area is represented. As a result, the flux-weighted averages are scaled directly to the global $\mathrm{H}_{2} \mathrm{O}$ flux, introducing the potential for sample bias.

This study thoroughly re-evaluates the surface-cycling of Mo and Re with particular attention on the re-assessment of world river averages and associated seawater 
response times (Chapter 2). This is achieved via a larger exorheic river subsample set of sufficient geographic diversity to permit the calculation of regional subtotals, thus minimizing the potential bias of any one sample. In addition, results from smaller rivers and tributaries provide insights into the global variability in Mo and Re river concentrations. We also present the first data for Re concentrations in hydrothermal fluids in an attempt to quantify this as-of-yet unknown flux.

This study also develops a method for Re stable isotope analysis $\left(\delta^{187} R e\right.$, Chapter 3). Previously demonstrated fractionation of Mo and $U$ due to changes in oxidation state (SIEBERT ET AL., 2003; Weyer ET AL., 2008) suggests that isotopic variability is also expected for Re which exhibits the same broad elemental behavior (MORFORD And EMERson, 1999). Though development of an analytical method for analysis of stable Re isotopic composition is motivated by similarities to Mo (and U), Re isotopes are as likely to be informative owing to its chemical differences from these elements. In particular: suboxic sediments are thought to be the largest Re sink; Re does not adsorb to Fe- or Mn-oxide crusts and shows the highest mobility under oxic weathering environments (Koide et AL., 1986; Crusius et AL., 1996; Morford AND Emerson, 1999; Peucker-Ehrenbrink and Hannigan, 2000; Jaffe et AL., 2002; Pierson-Wickmann et AL., 2003).

Lastly, a case study of natural Re isotope variations is evaluated within the context of a modern, redox variable weathering environment - an oxic weathering profile of the Devonian New Albany Shale, KY, USA (Chapter 4). 


\section{Bibliography}

Algeo, T. J. (2004), Can marine anoxic events draw down the trace element inventory of seawater?, Geology, 32: 1057-1060. [15, 91, 92, 175, 199]

Algeo, T. J. and Lyons, T. W. (2006), Mo-total organic carbon in modern anoxic marine environments: Implications for analysis of paleoredox and paleohydrographic conditions, Paleoceanography, 21: 23 pp. [16, 22, 23, 75, 176]

Arnold, G. L., Anbar, A. D., Barling, J., and Lyons, T. W. (2004), Molybdenum isotope evidence for widespread anoxia in mid-Proterozoic oceans, Science, 304: 87-90. [16, 22, 98, 104, 129, 175, 176, 186, 198, 199]

Berner, R. A. (1992), Comments on the role of marine sediment burial as a repository for anthropogenic $\mathrm{CO}_{2}$, Global Biogeochemical Cycles, 6: 1-2. [15]

Berner, R. A., Lasaga, A. C., and Garrels, R. M. (1983), The carbonate-silicate geochemical cycle and its effects on atmospheric carbon dioxide over the last 100 million years, American Journal of Science, 283: 641-683. [15, 21]

Bertine, K. K. and Turekian, K. K. (1973), Molybdenum in marine deposits, Geochimica et Cosmochimica Acta, 37: 1415-1434. [15, 16, 21, 23, 24, 26, 65, 69, 70, 71, $74,76,91,94,95,102,197]$

Colodner, D., Sachs, J., Ravizza, G., Turekian, K., Edmond, J., and Boyle, E. (1993), The geochemical cycle of rhenium: a reconnaissance, Earth and Planetary Science Letters, 117: 205-221. [15, 16, 21, 22, 23, 24, 26, 65, 69, 70, 71, 76, 79, 86, 87, 91, 92, 94, 95, 96, 102, 150, 151, 197, 198]

Crusius, J., Calvert, S., Pederson, T., and Sage, D. (1996), Rhenium and molybdenum enrichments in sediments as indicators of oxic, suboxic and sulfidic conditions of deposition, Earth and Planetary Science Letters, 145: 65-78. [15, 17, 21, 22, 75, 92, 176, 186, 198] 
Emerson, S. R. and Huested, S. S. (1991), Ocean anoxia and the concentrations of molybdenum and vanadium in seawater, Marine Chemistry, 34: 177-196. [15, 92, 199]

Jaffe, L. A., Peucker-Ehrenbrink, B., and Petsch, S. T. (2002), Mobility of rhenium, platinum group elements and organic carbon during black shale weathering, Earth and Planetary Science Letters, 198: 339-353. [17, 21, 76, 176, 177, 178, 179, 181, $183,186,198]$

Koide, M., Hodge, V. F., Yang, J. S., Stallard, M., Goldberg, E. G., Calhoun, J., and Bertine, K. K. (1986), Some comparative marine chemistries of rhenium, gold, silver and molybdenum, Applied Geochemistry, 1: 705-714. [17, 75, 76, 88, 176, $179,198,199]$

Morford, J. L. and Emerson, S. (1999), The geochemistry of redox sensitive trace metals in sediments, Geochimica et Cosmochimica Acta, 63: 1735-1750. [15, 16, $17,21,22,23,75,91,92,96,175,198,200]$

Neubert, N., Nägler, T. F., and Böttcher, M. E. (2008), Sulfidity controls molybdenum isotope fractionation into euxinic sediments: Evidence from the modern Black Sea, Geology, 36: 775-778. [16, 22, 186]

Pearce, C. R., Cohen, A. S., Coe, A. L., and Burton, K. W. (2008), Molybdenum isotope evidence for global ocean anoxia coupled with perturbations to the carbon cycle during the Early Jurassic, Geology, 36: 231-234. [16, 93, 98, 175, 199]

Peucker-Ehrenbrink, B. and Hannigan, R. E. (2000), Effects of black shale weathering on the mobility of rhenium and platinum group elements, Geology, 28: 475-478. $[17,76,78,150,151,176,179,198]$

Pierson-Wickmann, A.-C., Reisberg, L., and France-Lanord, C. (2003), Behavior of Re and Os during low-temperature alteration: results from Himalayan soils and 
altered black shales during low-temperature alteration: results from Himalayan soils and altered black shales, Geochimica et Cosmochimica Acta, 66: 1539-1548. $[17,76,87,176,179,198]$

Poulson, R. L., Siebert, C., McManus, J., and Berelson, W. M. (2006), Authigenic molybdenum isotope signatures in marine sediments, Geology, 34: 617-620. [16, $22,91,200]$

Ravizza, G. E. and Turekian, K. K. (1989), Application of the ${ }^{187}$ Re- ${ }^{187}$ Os system to black shale geochronometry, Geochimica et Cosmochimica Acta, 53: 3257-3262. $[15,78,150,151]$

Ravizza, G. E., Turekian, K. K., and Hay, B. J. (1991), The geochemistry of rhenium and osmium in recent sediments from the Black Sea, Geochimica et Cosmochimica Acta, 55: 3741-3752. [15, 75, 150, 151, 198]

Siebert, C., Nägler, T. F., von Blanckenburg, F., and Kramers, J. D. (2003), Molybdenum isotope records as a potential new proxy for paleoceanography, Earth and Planetary Science Letters, 211: 159-171. [15, 16, 17, 22, 76, 91, 92, 94, 98, 102, $104,176,179,186,199]$

Urey, H. C. (1952), The Planets, Their Origin and Development, Yale University Press, 245 pp. [15, 21]

Walker, L. M., Hays, P. B., and Kasting, J. F. (1981), A negative feedback mechanism for long-term stabilization of earth's surface temperature, Journal of Geophysical Research, 86: 9776-9782. [15, 21]

Weyer, S., Anbar, A. D., Gerdes, A., Gordon, G. W., Algeo, T. J., and Boyle, E. A. (2008), Natural fractionation of ${ }^{238} \mathrm{U} /{ }^{235} \mathrm{U}$, Geochimica et Cosmochimica Acta, 72 : 345-359. [16, 17, 176, 199] 


\section{Chapter 2}

\section{A reassessment of molybdenum and rhenium surface cycling}

\section{$2.1 \quad$ Introduction}

Along with the weathering of $\mathrm{Ca}$ - and $\mathrm{Mg}$-silicates, the burial of organic carbon $\left(\mathrm{C}_{\text {org }}\right)$ in sediments represents a major sink of atmospheric carbon dioxide $\left(\mathrm{CO}_{2}\right)$ (UREY,

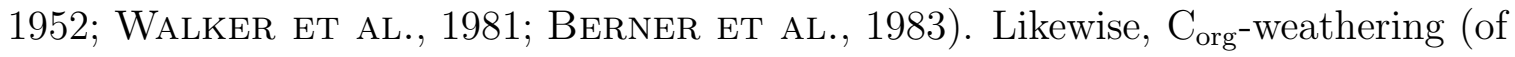
which the burning of fossil fuels can be considered an anthropogenic "short-circuit") is a source of atmospheric $\mathrm{CO}_{2}$. The association between $\mathrm{C}_{\text {org }}$ and metals such as $\mathrm{V}, \mathrm{Cr}$, Zn, Mo, Cd, Re, and U has been widely documented for both modern and ancient sediments, and these metals are used to infer the redox characteristics of the depositional and diagenetic environments (Bertine And Turekian, 1973; KlinkHammer And Palmer, 1991; Calvert and Pederson, 1993; Colodner et Al., 1993a; CruSiUs Et AL., 1996; Quinby-Hunt And Wilde, 1996; Morford And Emerson, 1999; Jaffe et AL., 2002; Tribovillard Et AL., 2006; Morford ET AL., 2007). These metals offer another way of evaluating $\mathrm{C}_{\text {org }}$ cycling throughout geologic time, with Mo and Re being particularly valuable as they show minimal detrital influence, exhibit the greatest enrichment in reducing sediments, are conservative in seawater, 
and have residence times sufficiently long to yield globally-integrated information (Morris, 1975; Collier, 1985; Anbar et Al., 1992; Colodner et Al., 1993a; Jones and Manning, 1994; Crusius et AL., 1996; Morford and Emerson, 1999; TRIBOVILlaRd Et AL., 2006).

Though redox sensitive metals were once used in a mostly binary fashion, in which their presence above detrital background denotes a reducing environment and background levels indicate an oxidizing one, their application to the palaeoenvironment has recently become more specific and quantitative. For example, Algeo And Lyons (2006) use the relationship between Mo and $\mathrm{C}_{\text {org }}$ to quantify the concentration of $\mathrm{H}_{2} \mathrm{~S}$ in bottom waters. Stable isotope fractionation of Mo has also been developed in hopes of formulating an indicator of palaeoredox conditions (SiEBERT ET AL., 2003; Arnold et Al., 2004; NäGler et Al., 2005; Poulson et Al., 2006; Neubert ET AL., 2008). Likewise, stable isotopes of Re can vary systematically across a redox gradient (Miller ET AL., 2008).

The increasingly informative use of Mo and Re, and their quantitative application to the palaeoenvironment depends on understanding of their present-day cycling in near-surface reservoirs. This study re-evaluates the surface cycling of Re and Mo with particular attention devoted to the riverine fluxes of these metals to seawater, and the resulting seawater response time.

\subsection{Background: surface cycling of Mo and Re}

Both Mo and Re are present in modern seawater in highly oxidized states - Mo(VI) and $\mathrm{Re}(\mathrm{VII})$ - as the respective oxyanions molybdate and perrhenate $-\mathrm{MoO}_{4}^{2-}$ and $\mathrm{ReO}_{4}^{-}$(Łetowski et AL., 1966; Brookins, 1986). Despite the incorporation of Mo into nitrogenase (ANBAR AND KNOLL, 2002) and the bio-accumulation of Re in certain seaweeds (Fukai And MeInke, 1962; YAng, 1991) both elements are considered to be conservative in seawater, and do not exhibit "nutrient-like" behavior 
(Morris, 1975; Collier, 1985; Anbar et Al., 1992; Colodner et Al., 1993a; TuIT, 2003). The concentration of Mo in seawater is $107 \mathrm{nM}$; that of Re is $40 \mathrm{pmol}$ $\mathrm{kg}^{-1}$ (Morris, 1975; Collier, 1985; Koide Et AL., 1987; Anbar Et Al., 1992; Colodner ET AL., 1993a, 1995). Assuming an average seawater density of $1.028 \mathrm{~g}$ $\mathrm{cm}^{-3}$ — after Montgomery (1958); Millero And Poisson (1981) — the resulting Mo and Re concentrations are $104 \mathrm{nmol} \mathrm{kg}^{-1}$ and $41 \mathrm{pM}$.

Fluxes of these metals out of seawater are very poorly constrained (MORFORD AND EMERSON, 1999), and residence times are determined using fluxes to seawater, thought to be dominated by dissolved riverine input. The world river average Mo concentration is currently thought to be $4.5 \mathrm{nmol} \mathrm{kg}^{-1}$ (BERTINE AND TUREKIAN, 1973). The world river average for Re is cited as $2.1 \mathrm{pmol} \mathrm{kg}^{-1}$ (COLODNER ET AL., 1993a). Using an oceanic volume of $1.35 \times 10^{21} \mathrm{~L}($ MenARD AND SMITH, 1966) and global river water flux of $3.86 \times 10^{16} \mathrm{Lyr}^{-1}$, (FEKETE ET AL., 2002), the Mo and Re world river averages correspond to seawater response times of $8.7 \times 10^{5} \mathrm{yr}$ and 7.2 $\times 10^{5} \mathrm{yr}$; these are similar to reported response times of $8.0 \times 10^{5} \mathrm{yr}$ (MORFORD AND Emerson, 1999) and $7.5 \times 10^{5}$ yr (COLODNer ET AL., 1993a).

Using hydrothermal water flux values from ELDERFIELD AND SCHUlTz (1996) and Mo concentrations from Metz And Trefry (2000), WheAt ET AL. (2002) estimate respective high- and low-temperature hydrothermal Mo fluxes of about 1\% and $13 \%$ the Mo riverine flux. Incorporation of this $14 \%$ flux increase (relative to the global river Mo flux) decreases the residence time of Mo in seawater to $\sim 7.6 \times 10^{5}$ yr. There are no equivalent published data for Re in hydrothermal fluids though RAVizzA ET AL. (1996) consider hydrothermal fluxes to be unimportant.

The global river fluxes of Mo and Re are currently assumed to represent $88 \%$ and $100 \%$ of the respective fluxes of these elements to seawater. Though these fluxes are crucial components of recent mass balance and modeling studies (MORFORD AND Emerson, 1999; Algeo AND LyOns, 2006), they are relatively poorly constrained. The world river average for Mo is based on three rivers - Amazon, Congo, and Maipo 
(Bertine And Turekian, 1973) - while that of Re is based on four - Amazon, Orinoco, Brahmaputra, and Ganges (Colodner et Al., 1993a). Assuming a global riverine water flux of $3.86 \times 10^{16} \mathrm{Lyr}^{-1}$ (FEKETE ET AL., 2002), these rivers represent 22 and $24 \%$ of the global runoff. While these proportions are not insignificant, both are heavily biased by the Amazon which accounts for 76 and $85 \%$ of the riverine flux used in Mo and Re river average determinations despite that it only represents $17 \%$ of the global river water flux (Meybeck and Ragu, 1995; Fekete et AL., 2002).

The principal aim of this study is to re-evaluate the world river average concentrations and oceanic residence times of Mo and Re. The potential for anthropogenic contributions of these elements to seawater will also be considered.

\subsection{Materials and Methods}

\subsubsection{Sample collection}

Because of the small sample sets used to calculate Mo and Re global river average concentrations and fluxes and the paucity of data on riverine concentrations of these elements generally, this study seeks not only to account for a larger proportion of the global river water flux, but also to obtain a sense of the range of Mo and Re concentrations. As such we present data not only for large exorheic rivers, but also for many smaller rivers and tributaries from around the world (Fig. 2-1). Such a study is collaborative by necessity, and samples were collected over several years by the authors and many other collaborators.

Samples were taken under metal free conditions. The collection vessels, usually a teflon dipper or low density polyethylene (LDPE) bottle, were rinsed with sample material prior to collection of the sample aliquot. Samples were pressure filtered using new, sterile plastic syringes (HSW, NORM-JECT ${ }^{\circledR}$ ) and sterile plastic filter

cartridges (Millipore ${ }^{\circledR}$, Sterivex ${ }^{\mathrm{TM}}, 0.22 \mu \mathrm{m}$ or $0.45 \mu \mathrm{m}$ ) into certified clean $125 \mathrm{~mL}$ high-density polyethylene (HDPE, EP Scientific Products) or into acid-cleaned 30, 60, 


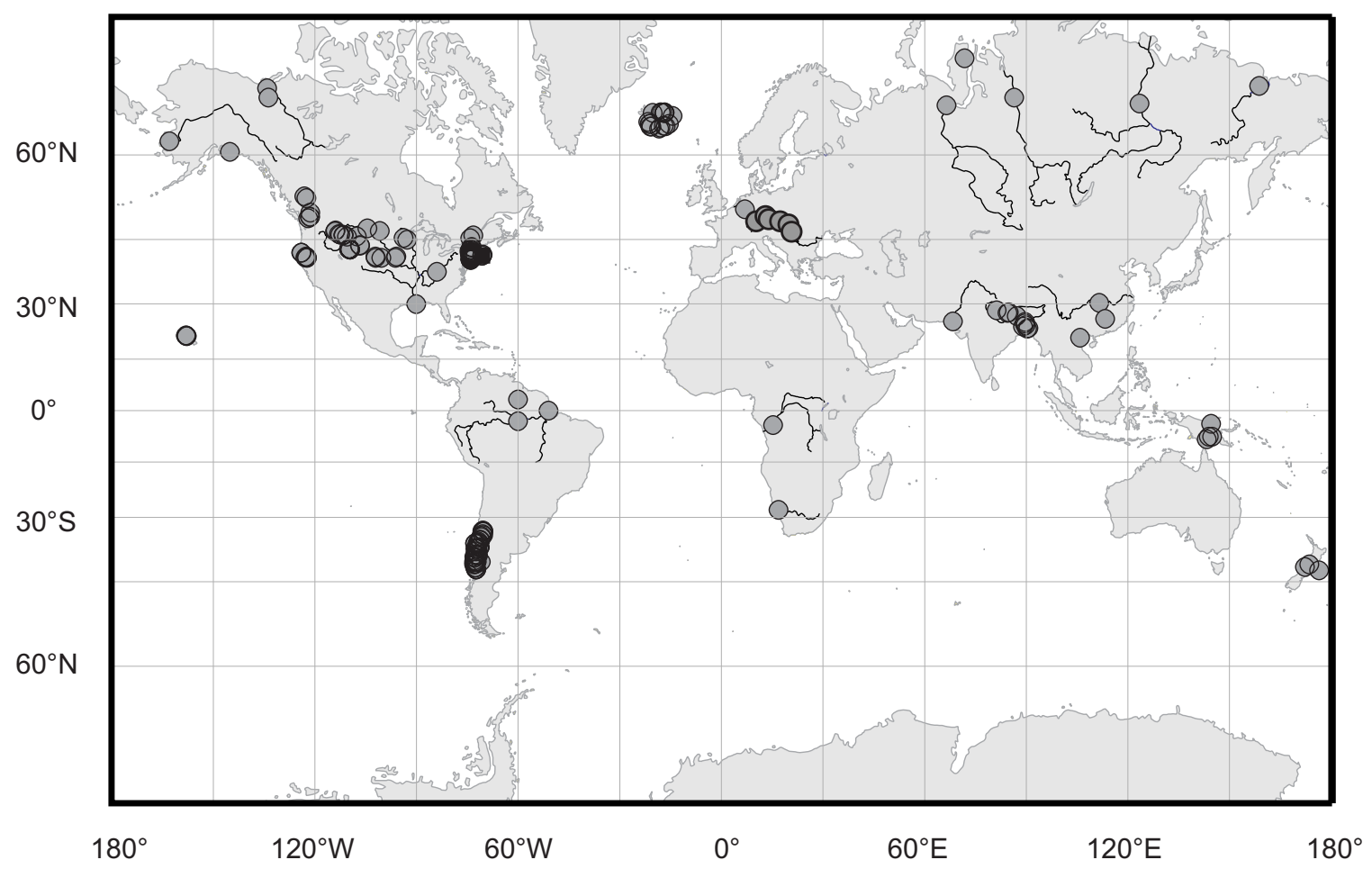

Figure 2-1: Sample locations for rivers examined in this study 
or $120 \mathrm{~mL}$ LDPE bottles (Nalgene). Syringes, filter cartridges, and bottles were also pre-treated with sample material before being used to process the collected sample aliquot. All items were single use with the exception of the collection vessel. In general, samples were not acidified, and were kept refrigerated at about $10^{\circ} \mathrm{C}$.

\subsubsection{Mo and Re concentrations}

Concentrations of Mo and Re were determined by isotope dilution inductively-coupled plasma mass spectrometry (ID-ICPMS). Approximately $20 \mathrm{~g}$ of sample was weighed into a clean bottle and spiked with ${ }^{95} \mathrm{Mo}$ and ${ }^{185} \mathrm{Re}$ spikes in $5 \% \mathrm{HNO}_{3}$, before being equilibrated for 2 days in an oven at $60^{\circ} \mathrm{C}$.

Column chromatography was done using $1 \mathrm{~mL}$ of Biorad AG $1 \times 8,100-200$ mesh anion resin (MORGAN ET AL., 1991) that had been pre-cleaned with $25 \%$ reagent grade $\mathrm{HCl}$ then $25 \%$ reagent grade $\mathrm{HNO}_{3}$. After loading on the column, samples are rinsed with $15 \mathrm{~mL} 0.5 \mathrm{~mol} \mathrm{~L}^{-1} \mathrm{HNO}_{3}$, then eluted with $10 \mathrm{~mL}$ of $4 \mathrm{~mol} \mathrm{~L}^{-1} \mathrm{HNO}_{3}$. Molybdenum is typically three orders of magnitude more abundant in natural waters than Re (Kharkar et al., 1968; Bertine and Turekian, 1973; Morris, 1975; Collier, 1985; Anbar et Al., 1992; Colodner et Al., 1993a); chromatography was therefore optimized for the quantitative recovery of Re. Recovery of Mo is not quantitative, though it is sufficient to obtain Mo ion beams larger than those of Re. Samples were dried down at $80^{\circ} \mathrm{C}$, taken up with a few drops $16 \mathrm{~mol} \mathrm{~L}^{-1} \mathrm{HNO}_{3}$ and $30 \% \mathrm{H}_{2} \mathrm{O}_{2}$ and dried down again to promote oxidation of resin-derived organics and ${ }^{187}$ Os. Samples were taken up in $5 \% \mathrm{~mol} \mathrm{~L}^{-1} \mathrm{HNO}_{3}$ for mass spectrometry.

All Mo and Re analyses were performed with the ELEMENT 2 mass spectrometer (Thermo Fisher Scientific) in the WHOI plasma facility. Samples were introduced using a PFA MicroFlow nebulizer (Elemental Scientific Incorporated), a quartz spray chamber, and regular cones. Masses 95 (Mo), 97 (Mo), 98 (Mo), 185 (Re), 187 (Re), $188(\mathrm{Os})$, and $101(\mathrm{Ru})$ were scanned 100 times in low mass resolution; each nuclide was counted for 2 seconds total. Mass 188 count rates were uniformly low and no 
correction was made for ${ }^{187}$ Os. Acid blanks and standards are analyzed every five or six samples to correct for blank contributions and instrumental mass fractionation, using natural Mo and Re isotopic ratios (Rosman AND TAYLOR, 1998).

Count-rates for non-spike isotopes (e.g. ${ }^{187} \mathrm{Re}$ and ${ }^{98} \mathrm{Mo}$ ) range across many orders of magnitude. In general uncertainties decrease with increasing signal intensity according to an inverse square root law, as one would expect for counting statistics. However measured uncertainties are typically about $5 \times$ the counting minimum $(1 / \sqrt{N})$, though in some cases (rinse-acid blanks) can exceed $100 \times$.

Uncertainties of Mo and Re concentration measurements are estimated using multiple $(\mathrm{n}=12)$ sample aliquot processing and analysis of a St. Lawrence sample from Côteau du Lac, Québec, Canada. Concentrations of Mo and Re varied by 6.2 and $4.6 \%$ (2 s.d.), respectively. This is similar to the Re concentration uncertainty of $4 \%$ reported by COLODNER ET AL. (1993b).

\subsubsection{Cation concentrations}

Concentrations of major $\left(\mathrm{Na}^{+}, \mathrm{Mg}^{+}, \mathrm{K}^{+}\right.$and $\left.\mathrm{Ca}^{+}\right)$as well as selected minor and trace cations $\left(\mathrm{Rb}^{+}, \mathrm{Sr}^{+}\right.$, and $\left.\mathrm{Ba}^{+}\right)$were determined by ICPMS. Wherever feasible cation analyses were done by ID-ICPMS. Isotope dilution (ID) determination of $\mathrm{Na}$ is not possible as it is mono-isotopic; ID determination of $\mathrm{K}$ is complicated by significant up-mass tailing of ${ }^{20} \mathrm{Ar}_{2}^{+}$on to ${ }^{41} \mathrm{~K}$; ID determination of both $\mathrm{Rb}$ and $\mathrm{Sr}$ would have doubled the number of analyses due to the isobars at mass 87. Concentrations of these three elements are done by standard-calibration ICPMS using ${ }^{23} \mathrm{Na},{ }^{39} \mathrm{~K}$, and ${ }^{85} \mathrm{Rb}$.

Sample sub-aliquots of one $\mathrm{mL}$ were spiked with mixed ${ }^{25} \mathrm{Mg}_{-}{ }^{135} \mathrm{Ba}$ and ${ }^{42} \mathrm{Ca}_{-}{ }^{84} \mathrm{Sr}$ spikes, before being diluted 10-fold with $5 \% \mathrm{HNO}_{3}$. Samples were heated at $60^{\circ} \mathrm{C}$ for 48 hours to allow for spike-sample equilibration before analysis. No purification chemistry was done.

Cation analyses were performed with the ELEMENT 2 mass spectrometer (Thermo 
Fisher Scientific) in the WHOI plasma facility. Samples were introduced using a PFA MicroFlow nebulizer (Elemental Scientific Incorporated), a quartz spray chamber, and regular cones. Analyses are done in low, medium, and high mass resolution. Low resolution evaluates masses $23(\mathrm{Na}), 24(\mathrm{Mg}), 25(\mathrm{Mg}), 26(\mathrm{Mg}), 82(\mathrm{Kr}), 83(\mathrm{Kr}), 84$ (Kr+Sr), $85(\mathrm{Rb}), 86(\mathrm{Sr}), 87(\mathrm{Sr}+\mathrm{Rb}), 88(\mathrm{Sr}), 135(\mathrm{Ba}), 137(\mathrm{Ba})$, and $138(\mathrm{Ba})$; as with Mo and Re, ion beam intensities were measured across 20 channels of the central $5 \%$ of the peak. Medium resolution evaluates masses 42 (Ca), 43 (Ca), 44 (Ca), and 48 (Ca) by integrating the ion beam intensity of the entire peak. High resolution evaluates masses $23(\mathrm{Na}), 24(\mathrm{Mg}), 25(\mathrm{Mg}), 26(\mathrm{Mg})$, and $39(\mathrm{~K})$, as whole peak integrations.

At the beginning of an analytical sequence, five-point standard calibration curves of ${ }^{23} \mathrm{Na},{ }^{39} \mathrm{~K}$, and ${ }^{85} \mathrm{Rb}$ were constructed to evaluate these elements. Nuclide transmission in the ELEMENT 2 is a function of mass and closely follows a power law. Calibration curves showed a high degree of linearity $\left(\mathrm{r}^{2}>0.99\right)$ and cross the countinganalogue mode transition of the secondary electron multiplier (SEM). A typical analytical sequence ( 43 samples, 5 standards, 49 rinse acid blanks) takes $\sim 12$ hours. Standard analyses from during and after a sequence indicate no significant instrumental drift over the course of a sequence.

As an internal consistency check spike-unmixing of $\mathrm{Mg}, \mathrm{Ca}, \mathrm{Sr}$, and $\mathrm{Ba}$ was done with multiple isotope ratios $\left({ }^{25} \mathrm{Mg} /{ }^{24} \mathrm{Mg},{ }^{25} \mathrm{Mg} /{ }^{26} \mathrm{Mg},{ }^{42} \mathrm{Ca} /{ }^{43} \mathrm{Ca},{ }^{42} \mathrm{Ca} /{ }^{44} \mathrm{Ca}\right.$, ${ }^{42} \mathrm{Ca} /{ }^{48} \mathrm{Ca},{ }^{135} \mathrm{Ba} /{ }^{135} \mathrm{Ba}$, and $\left.{ }^{135} \mathrm{Ba} /{ }^{138} \mathrm{Ba}\right) .{ }^{23}$ Sodium was measured in both low and high resolution for the same reason; unfortunately this could not be done for $\mathrm{K}$ as proximity to the ${ }^{20} \mathrm{Ar}_{2}^{+}$peak restricts ${ }^{39} \mathrm{~K}$ acquisition to high resolution, or for $\mathrm{Rb}$ as even low-resolution intensities of ${ }^{85} \mathrm{Rb}$ were extremely low.

Uncertainty of cation measurements was estimated using multiple sample aliquot processing $(n=22)$ and analyses $(n=33)$ of a St. Lawrence sample from Côteau du Lac, Québec, Canada. Uncertainties of $\mathrm{Na}, \mathrm{Mg}, \mathrm{Ca}, \mathrm{K}, \mathrm{Rb}, \mathrm{Sr}$, and $\mathrm{Ba}$ are 14, 1.3, $10,16,15,20$, and $7 \%$ (2 s.d.) respectively. The uncertainty for Sr concentration is 
very large considering analyses were done by isotope dilution; this is due to the $60 \%$ contribution of ${ }^{84} \mathrm{Kr}$ to the mass 84 ion beam intensity $\left({ }^{84} \mathrm{Sr}\right.$ is the $\mathrm{Sr}$ spike isotope).

\subsubsection{Anion concentrations}

Concentrations of $\mathrm{Cl}^{-}$and $\mathrm{SO}_{4}^{2-}$ were determined by standard-calibration ion chromatography using a Dionex ${ }^{\circledR}$ DX-500 ion chromatography system comprised of an ED 40 Electrochemical Detector, a GP 50 Gradient Pump, an LC 30 Chromatography oven, an IonPac ${ }^{\circledR}$ AS15 4-mm chromatographic column and an ASRS ${ }^{\circledR}$-ULTRA II 4-mm suppressor.

Approximately $5 \mathrm{~mL}$ of sample fluid was loaded into a clean plastic Dionex vial, allowing three separate sample injections per vial. Samples were eluted with a 60:40\% (v/v) mixture of $50 \mathrm{mN} \mathrm{NaOH}$ and $\mathrm{H}_{2} \mathrm{O}$. Standards were analyzed after every nine sample injections, or about every 2 hours. Standards were used to construct a three point calibration curve. When sample concentrations were higher than the most concentrated standard, samples were diluted to within range of the calibration and reanalyzed. Sample concentrations were lower than the most dilute standard only twice; as the concentrations of these samples were very close to the lowest calibration point, it was assumed that the calibration provided accurate concentrations. Reproducibilities of $\mathrm{Cl}^{-}$and $\mathrm{SO}_{4}^{2-}$ for the most dilute standard were $3.4 \%$ and $4.3 \%$ respectively (2 s.d., $n=74)$; reproducibilities for a St. Lawrence River sample $(n=69)$ were similar to those of the nearest (most concentrated) calibrating standard. We cite a conservative uncertainty of $5 \%$ (2 s.d.) for $\mathrm{Cl}^{-}$and $\mathrm{SO}_{4}^{2-}$ concentrations. 


\subsection{Results}

Data for this study are presented in Tables $2.2-2.4$. Exorheic river data used to recalculate the world river average concentrations of Mo and Re are presented in Table 2.2. Chemical data (dissolved Re, Mo, Cl, $\mathrm{SO}_{4}, \mathrm{Na}, \mathrm{Mg}$, Ca, K, Rb, Sr, and Ba) for North American and Russian Arctic rivers, as well as for the Mississippi presented in Table 2.2 are determined by calculating flux weighted chemical averages using the time-series data presented in Table 2.1. The first concentration data for Re in hydrothermal fluids (high temperature, Manus Basin) are presented in Table 2.6; data for Mo are also presented. Hydrothermal data include fluid component data as well as concentration and mass-balance information for two categories of precipitating sulphide; all three components are necessary to reconstitute the original sampled fluid. The Mg concentration data originally presented in CRADDock (2008) used to correct for entrained seawater are also shown. Table 2.3 presents data for minor rivers and tributaries from around the globe in an attempt to capture the global variability of river Mo and Re concentration data. Profiles of the Mississippi (one profile) and Hudson River (six profiles) estuaries are presented in Table 2.5. Data for precipitation samples (Falmouth, MA, USA) and waters associated with mining (Berkeley Pit, Butte MT and Mansfeld, Germany) are shown in Table 2.4. 


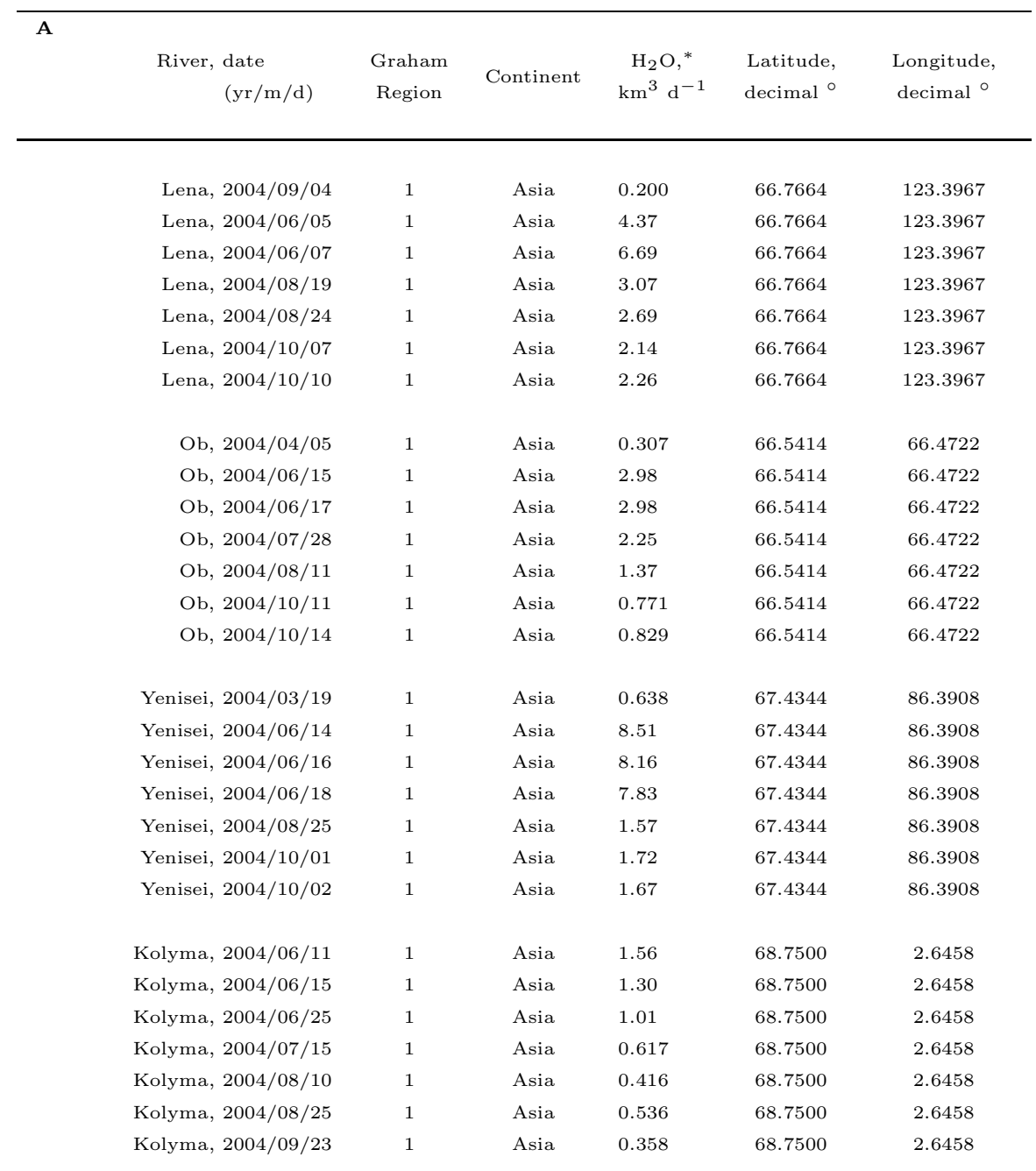




\begin{tabular}{|c|c|c|c|c|c|}
\hline $\begin{array}{l}\text { River, date } \\
\qquad(\mathrm{yr} / \mathrm{m} / \mathrm{d})\end{array}$ & $\begin{array}{l}\text { Graham } \\
\text { Region }\end{array}$ & Continent & $\begin{array}{c}\mathrm{H}_{2} \mathrm{O},{ }^{*} \\
\mathrm{~km}^{3} \mathrm{~d}^{-1}\end{array}$ & $\begin{array}{l}\text { Latitude, } \\
\text { decimal }\end{array}$ & $\begin{array}{c}\text { Longitude, } \\
\text { decimal }\end{array}$ \\
\hline Mackenzie, 2004/03/24 & 2 & N Amer & 0.327 & 67.4521 & -133.7389 \\
\hline Mackenzie, 2004/06/17 & 2 & N Amer & 1.58 & 67.4521 & -133.7389 \\
\hline Mackenzie, 2004/06/22 & 2 & N Amer & 1.50 & 67.4521 & -133.7389 \\
\hline Mackenzie, 2004/07/13 & 2 & N Amer & 1.08 & 67.4521 & -133.7389 \\
\hline Mackenzie, 2004/08/04 & 2 & N Amer & 1.07 & 67.4521 & -133.7389 \\
\hline Mackenzie, 2004/08/25 & 2 & N Amer & 0.754 & 67.4521 & -133.7389 \\
\hline Mackenzie, 2004/08/09 & 2 & N Amer & 0.758 & 67.4521 & -133.7389 \\
\hline Yukon, 2004/04/07 & 10 & N Amer & 0.1138 & 61.9486 & -162.9077 \\
\hline Yukon, 2004/05/26 & 10 & N Amer & 1.282 & 61.9486 & -162.9077 \\
\hline Yukon, 2004/06/15 & 10 & N Amer & 1.563 & 61.9486 & -162.9077 \\
\hline Yukon, 2004/06/29 & 10 & N Amer & 1.258 & 61.9486 & -162.9077 \\
\hline Yukon, 2004/07/19 & 10 & N Amer & 0.8685 & 61.9486 & -162.9077 \\
\hline Yukon, 2004/08/18 & 10 & N Amer & 0.8172 & 61.9486 & -162.9077 \\
\hline Yukon, 2004/09/22 & 10 & N Amer & 0.4942 & 61.9486 & -162.9077 \\
\hline Lena, 2007/05/26 & 1 & Asia & 2.08 & 66.7664 & 123.3967 \\
\hline Lena, 2007/05/27 & 1 & Asia & 3.15 & 66.7664 & 123.3967 \\
\hline Lena, 2007/05/28 & 1 & Asia & 4.94 & 66.7664 & 123.3967 \\
\hline Lena, 2007/05/29 & 1 & Asia & 6.22 & 66.7664 & 123.3967 \\
\hline Lena, 2007/05/30 & 1 & Asia & 7.01 & 66.7664 & 123.3967 \\
\hline Lena, 2007/05/31 & 1 & Asia & 7.78 & 66.7664 & 123.3967 \\
\hline Lena, 2007/06/02 & 1 & Asia & 8.64 & 66.7664 & 123.3967 \\
\hline Lena, 2007/06/02 & 1 & Asia & 8.50 & 66.7664 & 123.3967 \\
\hline Lena, 2007/06/04 & 1 & Asia & 8.03 & 66.7664 & 123.3967 \\
\hline Lena, 2007/06/05 & 1 & Asia & 9.50 & 66.7664 & 123.3967 \\
\hline Lena, 2007/06/06 & 1 & Asia & 8.99 & 66.7664 & 123.3967 \\
\hline Lena, 2007/06/08 & 1 & Asia & 7.67 & 66.7664 & 123.3967 \\
\hline Lena, 2007/06/09 & 1 & Asia & 7.49 & 66.7664 & 123.3967 \\
\hline Lena, 2007/06/10 & 1 & Asia & 7.38 & 66.7664 & 123.3967 \\
\hline Lena, 2007/06/11 & 1 & Asia & 7.23 & 66.7664 & 123.3967 \\
\hline
\end{tabular}




\begin{tabular}{|c|c|c|c|c|c|}
\hline $\begin{array}{l}\text { River, date } \\
\qquad(\mathrm{yr} / \mathrm{m} / \mathrm{d})\end{array}$ & $\begin{array}{l}\text { Graham } \\
\text { Region }\end{array}$ & Continent & $\begin{array}{c}\mathrm{H}_{2} \mathrm{O},{ }^{*} \\
\mathrm{~km}^{3} \mathrm{~d}^{-1}\end{array}$ & $\begin{array}{l}\text { Latitude, } \\
\text { decimal }\end{array}$ & $\begin{array}{l}\text { Longitude, } \\
\text { decimal }\end{array}$ \\
\hline $\mathrm{Ob}, 2007 / 05 / 29$ & 1 & Asia & 3.21 & 66.5414 & 66.4722 \\
\hline $\mathrm{Ob}, 2007 / 05 / 30$ & 1 & Asia & 3.20 & 66.5414 & 66.4722 \\
\hline $\mathrm{Ob}, 2007 / 05 / 31$ & 1 & Asia & 3.14 & 66.5414 & 66.4722 \\
\hline $\mathrm{Ob}, 2007 / 06 / 01$ & 1 & Asia & 3.14 & 66.5414 & 66.4722 \\
\hline $\mathrm{Ob}, 2007 / 06 / 02$ & 1 & Asia & 3.13 & 66.5414 & 66.4722 \\
\hline Ob, 2007/06/03 & 1 & Asia & 3.11 & 66.5414 & 66.4722 \\
\hline Ob, 2007/06/04 & 1 & Asia & 3.08 & 66.5414 & 66.4722 \\
\hline Ob, 2007/06/05 & 1 & Asia & 3.03 & 66.5414 & 66.4722 \\
\hline $\mathrm{Ob}, 2007 / 06 / 06$ & 1 & Asia & 3.02 & 66.5414 & 66.4722 \\
\hline $\mathrm{Ob}, 2007 / 06 / 07$ & 1 & Asia & 3.02 & 66.5414 & 66.4722 \\
\hline Ob, 2007/06/08 & 1 & Asia & 3.02 & 66.5414 & 66.4722 \\
\hline Ob, 2007/06/09 & 1 & Asia & 3.01 & 66.5414 & 66.4722 \\
\hline Ob, 2007/06/10 & 1 & Asia & 3.00 & 66.5414 & 66.4722 \\
\hline $\mathrm{Ob}, 2007 / 06 / 11$ & 1 & Asia & 3.00 & 66.5414 & 66.4722 \\
\hline Ob, 2007/06/12 & 1 & Asia & 3.01 & 66.5414 & 66.4722 \\
\hline Ob, 2007/06/13 & 1 & Asia & 3.01 & 66.5414 & 66.4722 \\
\hline Ob, 2007/06/14 & 1 & Asia & 3.01 & 66.5414 & 66.4722 \\
\hline Ob, 2007/06/15 & 1 & Asia & 3.08 & 66.5414 & 66.4722 \\
\hline $\mathrm{Ob}, 2007 / 06 / 16$ & 1 & Asia & 3.10 & 66.5414 & 66.4722 \\
\hline Ob, 2007/06/17 & 1 & Asia & 3.11 & 66.5414 & 66.4722 \\
\hline Upper Mississippi, 2004/02/11 & 3 & $\mathrm{~N}$ Amer & 0.0864 & 38.8827 & -90.1882 \\
\hline Upper Mississippi, 2004/04/14 & 3 & N Amer & 0.409 & 38.8827 & -90.1882 \\
\hline Upper Mississippi, 2004/07/16 & 3 & N Amer & 0.416 & 38.8827 & -90.1882 \\
\hline Upper Mississippi, 2004/11/12 & 3 & N Amer & 0.294 & 38.8827 & -90.1882 \\
\hline Missouri, 2004/02/10 & 3 & N Amer & 0.0785 & 38.5610 & -92.0092 \\
\hline Missouri, 2004/04/14 & 3 & N Amer & 0.0785 & 38.5610 & -92.0092 \\
\hline Missouri, 2004/07/12 & 3 & N Amer & 0.204 & 38.5610 & -92.0092 \\
\hline Missouri, 2004/11/12 & 3 & N Amer & 0.153 & 38.5610 & -92.0092 \\
\hline Ohio, 2004/02/12 & 3 & N Amer & 2.14 & 37.0010 & -89.1638 \\
\hline Ohio, 2004/04/14 & 3 & N Amer & 0.484 & 37.0010 & -89.1638 \\
\hline Ohio, 2004/07/17 & 3 & $\mathrm{~N}$ Amer & 0.519 & 37.0010 & -89.1638 \\
\hline Ohio, 2004/11/13 & 3 & N Amer & 0.776 & 37.0010 & -89.1638 \\
\hline
\end{tabular}




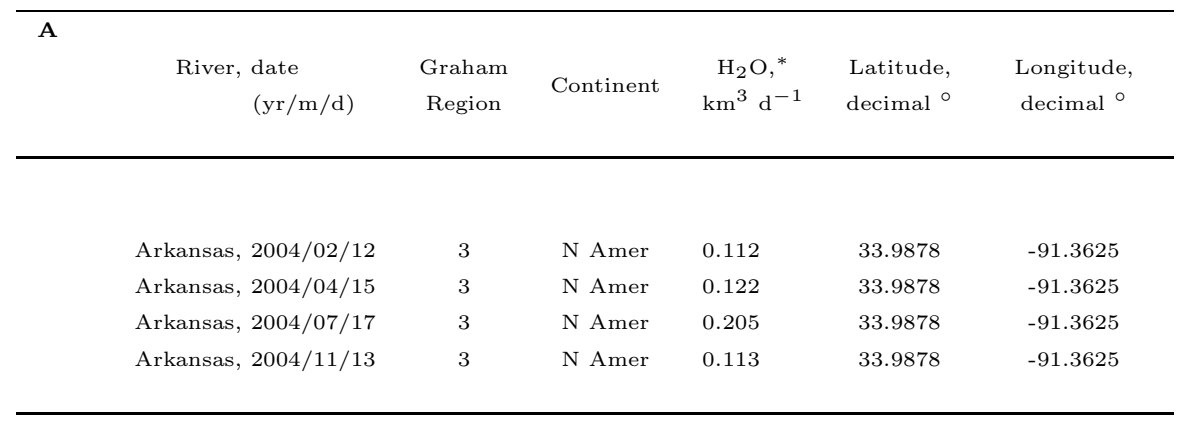

* Water flux data for Arctic rivers were taken from the University of New Hampshire's (UNH)

Arctic Regional, Integrated Hydrological Monitoring System (RIMS) website available at

http://rims.unh.edu/

Lena River at Kusur, Station Code 3821

Ob River at Salekhard, Station Code 11801

Yenisei River at Igarka, Station Code 9803

Kolyma River at Kolymskoye, Station Code 1802

Mackenzie River at Arctic Red River, Station Code 10LC014

Yukon River at Pilot Station AK, Station Code 15565447

Water flux data for Mississippi tributaries were taken from the United States Geological Survey

(USGS) National Water Information System (NWIS) available at http://waterdata.usgs.gov/nwis/

Upper Mississippi River at Grafton IL, Station ID 05587450

Missouri River at Hermann MO, Station ID 06934500

Ohio River at Metropolis IL, Station ID 03611500

Arkansas River at Murray Dam near Little Rock AK, Station ID 07263450

Table 2.1: A: River time-series samples, sampling dates, $\mathrm{H}_{2} \mathrm{O}$ fluxes, and sampling locations for Arctic rivers and Mississippi tributaries. 


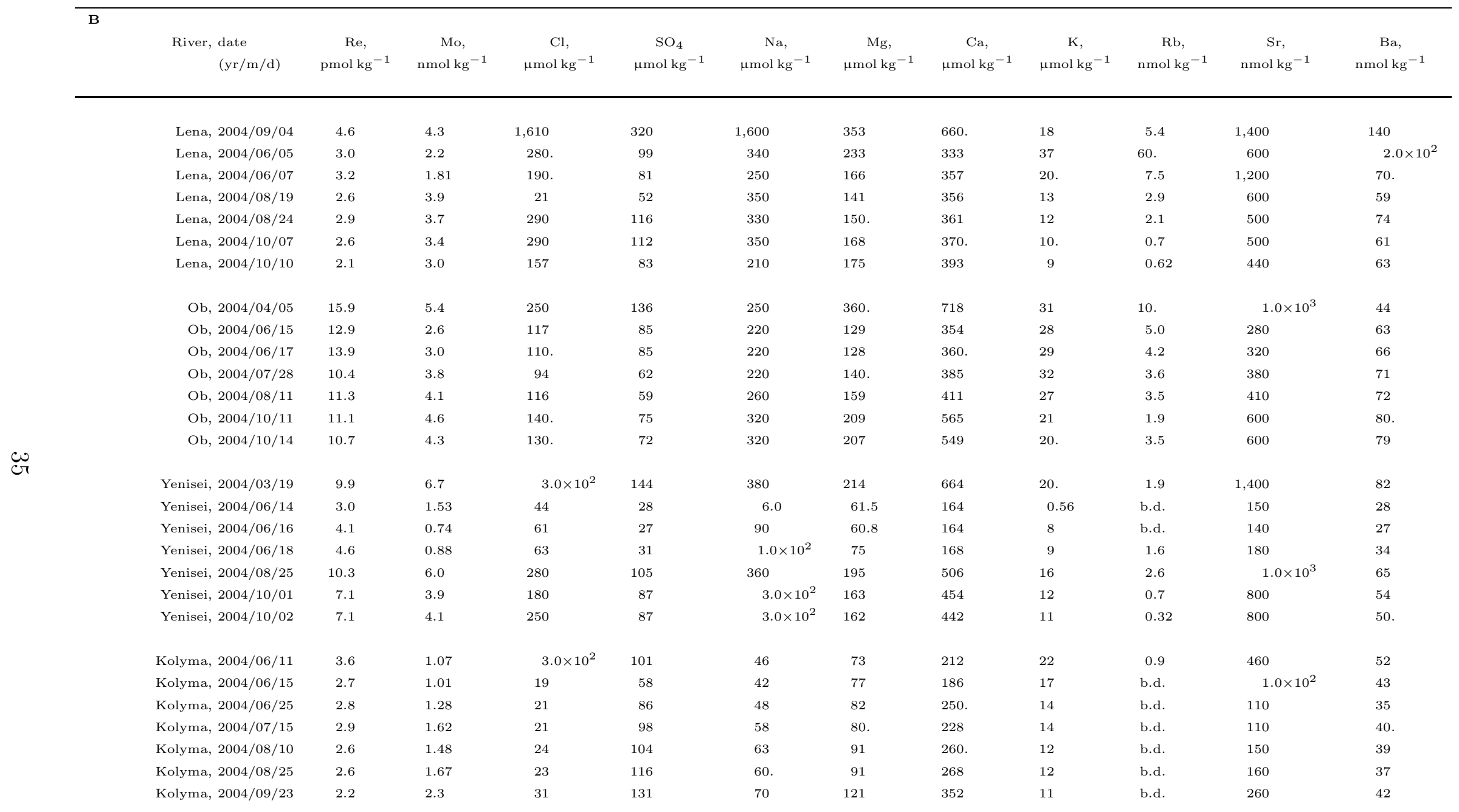




\begin{tabular}{|c|c|c|c|c|c|c|c|c|c|c|c|}
\hline $\begin{array}{l}\text { River, date } \\
\qquad(\mathrm{yr} / \mathrm{m} / \mathrm{d})\end{array}$ & $\begin{array}{c}\mathrm{Re}, \\
\mathrm{pmol} \mathrm{kg}^{-1}\end{array}$ & $\begin{array}{c}\text { Mo, } \\
\text { nmol kg }\end{array}$ & $\begin{array}{c}\mathrm{Cl}, \\
\mu \mathrm{mol} \mathrm{kg}\end{array}$ & $\begin{array}{c}\mathrm{SO}_{4} \\
\mu \mathrm{mol} \mathrm{kg}{ }^{-1}\end{array}$ & $\begin{array}{c}\mathrm{Na}, \\
\mu \mathrm{mol} \mathrm{kg}\end{array}$ & $\begin{array}{c}\mathrm{Mg}, \\
\mu \mathrm{mol} \mathrm{kg} \mathrm{kg}^{-1}\end{array}$ & $\begin{array}{c}\mathrm{Ca}, \\
\mu \mathrm{mol} \mathrm{kg}{ }^{-1}\end{array}$ & $\begin{array}{c}\mathrm{K}, \\
\mu \mathrm{mol} \mathrm{kg}\end{array}$ & $\begin{array}{c}\mathrm{Rb}, \\
\mathrm{nmol} \mathrm{kg}{ }^{-1}\end{array}$ & $\begin{array}{c}\mathrm{Sr}, \\
\mathrm{nmol} \mathrm{kg}\end{array}$ & $\begin{array}{c}\mathrm{Ba}, \\
\mathrm{nmol} \mathrm{kg}{ }^{-1}\end{array}$ \\
\hline Mackenzie, 2004/03/24 & 16.8 & 9.3 & 380 & 410 & $5.0 \times 10^{2}$ & 374 & 847 & 24 & 4.4 & 1,400 & 260 \\
\hline Mackenzie, 2004/06/17 & 14.9 & 9.0 & 143 & 310 & 240 & 307 & 817 & 20. & 5.4 & 1,200 & 250 \\
\hline Mackenzie, 2004/06/22 & 15.5 & 2.8 & 130. & 310 & 220 & 308 & 766 & 22 & 9 & $1.0 \times 10^{3}$ & $3.0 \times 10^{2}$ \\
\hline Mackenzie, 2004/07/13 & 16.2 & 10.4 & 181 & 360 & 290 & 336 & 868 & 20. & 4.7 & 1,100 & 240 \\
\hline Mackenzie, 2004/08/04 & 20. & 11.5 & 210 & $4.0 \times 10^{2}$ & 330 & 369 & 903 & 22 & 5.6 & 1,400 & 250 \\
\hline Mackenzie, 2004/08/25 & 15.8 & 13.0 & 240 & 410 & 370 & 412 & 980 & 28 & 19 & 1,600 & 380 \\
\hline Mackenzie, 2004/08/09 & 15.7 & 13.2 & 260 & 430 & $4.0 \times 10^{2}$ & 402 & 950 & 26 & 11 & 1,600 & 310 \\
\hline Yukon, 2004/04/07 & 12.0 & 13.6 & 79 & 280 & 160 & 332 & 818 & 33 & 13 & 700 & 140 \\
\hline Yukon, 2004/05/26 & 8.1 & 5.1 & 43 & 152 & 80 & 187 & 593 & 25 & 8 & 410 & 160 \\
\hline Yukon, 2004/06/15 & 10.8 & 7.6 & 42 & $2.0 \times 10^{2}$ & 70212 & 717 & 27 & 10 & .320 & 2 & $.0 \times 10^{2}$ \\
\hline Yukon, 2004/06/29 & 12.3 & 19 & 69 & 250 & $1.0 \times 10^{2}$ & 258 & 812 & 29 & 12 & 500 & 220 \\
\hline Yukon, 2004/07/19 & 15.6 & 13.9 & 74 & 310 & 140 & 322 & 800. & 54 & 35 & 700 & 260 \\
\hline Yukon, 2004/08/18 & 17.5 & 14.6 & 66 & $4.0 \times 10^{2}$ & 160 & 391 & 828 & 63 & 55 & 900 & 410 \\
\hline Yukon, 2004/09/22 & 17.8 & 14.1 & 85 & 390 & 160 & 381 & 970 & 42 & 19 & 900 & 250 \\
\hline Lena, $2007 / 05 / 26$ & 0.72 & 1.32 & 120. & 38 & 130 & 73 & 288 & 8 & 2.2 & 900 & 62 \\
\hline Lena, 2007/05/27 & 1.91 & 2.9 & 390 & 108 & 410 & 191 & 390. & 27 & 9 & 900 & 93 \\
\hline Lena, 2007/05/28 & 2.19 & 2.8 & 380 & 103 & 420 & 187 & 385 & 32 & 9 & 800 & 91 \\
\hline Lena, 2007/05/29 & 1.75 & 2.2 & 450 & 87 & 340 & 158 & 310. & 28 & 8 & 600 & 77 \\
\hline Lena, 2007/05/30 & 1.71 & 2.0 & 320 & 80. & 310 & 149 & 294 & 28 & 9 & 600 & 79 \\
\hline Lena, 2007/05/31 & 1.84 & 2.0 & 310 & 78 & 330 & 149 & 291 & 29 & 9 & 500 & 74 \\
\hline Lena, 2007/06/02 & 2.12 & 1.9 & 250 & 69 & 270 & 143 & 305 & 30. & 9 & 500 & 70. \\
\hline Lena, 2007/06/02 & 1.91 & 1.9 & 250 & 65 & 260 & 142 & 291 & 29 & 9 & 500 & 76 \\
\hline Lena, 2007/06/04 & 1.96 & 1.81 & 210 & 62 & 230 & 89 & 390. & 29 & 8 & 700 & 45 \\
\hline Lena, 2007/06/05 & 1.58 & 1.55 & 190 & 49 & 190 & 126 & 265 & 24 & 7 & 410 & 70. \\
\hline Lena, 2007/06/06 & 1.69 & 1.55 & 158 & 46 & 180 & 123 & 278 & 24 & 6 & 500 & 70. \\
\hline Lena, 2007/06/08 & 1.51 & 1.63 & 91 & 31 & 130 & 123 & 273 & 24 & 4.8 & 320 & 64 \\
\hline Lena, 2007/06/09 & 1.79 & 1.50 & 90. & 31 & 120 & 126 & 285 & 23 & 4.5 & 320 & 64 \\
\hline Lena, 2007/06/10 & 0.78 & 0.80 & 47 & 17.6 & 66 & 93 & 255 & 14 & 2.7 & 290 & 60. \\
\hline Lena, 2007/06/11 & 1.61 & 1.45 & 79 & 29 & $1.0 \times 10^{2}$ & 139 & 312 & 23 & 4.7 & 380 & 69 \\
\hline
\end{tabular}




\begin{tabular}{|c|c|c|c|c|c|c|c|c|c|c|c|}
\hline $\begin{array}{l}\text { River, date } \\
\qquad(\mathrm{yr} / \mathrm{m} / \mathrm{d})\end{array}$ & $\begin{array}{c}\text { Re, } \\
\text { pmol kg }\end{array}$ & $\begin{array}{c}\text { Mo, } \\
\text { nmol kg }\end{array}$ & $\begin{array}{c}\mathrm{Cl}, \\
\mu \mathrm{mol} \mathrm{kg}\end{array}$ & $\begin{array}{c}\mathrm{SO}_{4} \\
\mu \mathrm{mol} \mathrm{kg}\end{array}$ & $\begin{array}{c}\mathrm{Na}, \\
\mu \mathrm{mol} \mathrm{kg}{ }^{-1}\end{array}$ & $\begin{array}{c}\mathrm{Mg}, \\
\mu \mathrm{mol} \mathrm{kg} \mathrm{kg}^{-1}\end{array}$ & $\begin{array}{c}\mathrm{Ca}, \\
\mu \mathrm{mol} \mathrm{kg}\end{array}$ & $\begin{array}{c}\mathrm{K}, \\
\mu \mathrm{mol} \mathrm{kg}\end{array}$ & $\begin{array}{c}\mathrm{Rb}, \\
\mathrm{nmol} \mathrm{kg}{ }^{-1}\end{array}$ & $\begin{array}{c}\mathrm{Sr}, \\
\mathrm{nmol} \mathrm{kg}\end{array}$ & $\begin{array}{c}\mathrm{Ba}, \\
\mathrm{nmol} \mathrm{kg}^{-1}\end{array}$ \\
\hline Ob, 2007/05/29 & 2.09 & 0.89 & 50. & 19 & 80 & 49.4 & 136 & 13 & 3.0 & 80 & 33 \\
\hline Ob, 2007/05/30 & 1.57 & 0.56 & 97 & 12.8 & 68 & 21.0 & 174 & 13 & 3.5 & $1.0 \times 10^{3}$ & 20. \\
\hline $\mathrm{Ob}, 2007 / 05 / 31$ & 1.26 & 0.38 & 60. & 9.1 & 60. & 35.5 & 67.8 & 12 & 4.2 & $30 .$. & 23 \\
\hline Ob, 2007/06/01 & 1.57 & 0.44 & 55 & 10.3 & 70 & 39.0 & 71.5 & 14 & 5.6 & 33 & 26 \\
\hline Ob, 2007/06/02 & 1.48 & 0.60 & 67 & 11.0 & 55 & 31.7 & 75.3 & 10. & 2.7 & 39 & 28 \\
\hline Ob, 2007/06/03 & 1.41 & 0.36 & 102 & 7.7 & 63 & 32.9 & 53.6 & 13 & 4.7 & 15 & 22 \\
\hline Ob, 2007/06/04 & 1.14 & 0.35 & 54 & 8.6 & 62 & 32.8 & 57.5 & 11 & 3.4 & 17 & 25 \\
\hline Ob, 2007/06/05 & 2.1 & 0.53 & 31 & 13.1 & 66 & 40.6 & 73.2 & 12 & 3.3 & 30. & 31 \\
\hline Ob, 2007/06/06 & 0.82 & 0.44 & 79 & 7.2 & 55 & 30.4 & 53.8 & 10. & 3.8 & 12 & 22 \\
\hline Ob, 2007/06/07 & 1.30 & 0.53 & 34 & 11.4 & 65 & 37.2 & 68.9 & 12 & 5.1 & 31 & 27 \\
\hline Ob, 2007/06/08 & 4.9 & 0.43 & 48 & 10.3 & 50. & 32.4 & 55.6 & 10. & 3.0 & 16 & 26 \\
\hline Ob, 2007/06/09 & 7.5 & 0.47 & 53 & 10.8 & 58 & 33.5 & 58.8 & 10. & 3.0 & 18 & 30. \\
\hline $\mathrm{Ob}, 2007 / 06 / 10$ & 2.1 & 0.74 & 30. & 14.1 & 46 & 36.5 & 82.8 & 9 & 1.9 & 39 & 34 \\
\hline Ob, 2007/06/11 & 3.1 & 1.06 & 80. & 23 & 90 & 52.8 & 124 & 14 & 3.5 & 90 & 33 \\
\hline Ob, 2007/06/12 & 0.78 & 0.22 & 20. & 3.2 & 31 & 17.1 & 40.7 & 5.2 & 1.1 & b.d. & 21 \\
\hline Ob, $2007 / 06 / 13$ & 1.35 & 0.61 & 51 & 12.7 & 70 & 38.2 & 80.3 & 11 & 3.5 & 42 & 26 \\
\hline Ob, $2007 / 06 / 14$ & 1.9 & 0.47 & 63 & 10.8 & 70 & 37.8 & 65.3 & 10. & 4.2 & 30. & 31 \\
\hline Ob, $2007 / 06 / 15$ & 0.96 & 0.49 & 36 & 11.4 & 80 & 39.6 & 84.3 & 13 & b.d. & 30. & 16 \\
\hline Ob, 2007/06/16 & 1.60 & 0.67 & 40. & 13.9 & 70 & 41.0 & 92.3 & 10. & 3.2 & 45 & 29 \\
\hline $\mathrm{Ob}, 2007 / 06 / 17$ & 1.43 & 0.68 & 25 & 6.2 & 40. & 24.1 & 57.9 & 5.8 & 1.3 & 9 & 23 \\
\hline Upper Mississippi, 2004/02/11 & 106 & 26 & $1.20 \times 10^{3}$ & 440 & -- & -- & -- & -- & -- & -- & -- \\
\hline Upper Mississippi, 2004/04/14 & 45 & 11.0 & 570 & 270 & -- & -- & -- & -- & -- & -- & -- \\
\hline Upper Mississippi, 2004/07/16 & 112 & 25 & 550 & 340 & -- & -- & -- & -- & -- & -- & -- \\
\hline Upper Mississippi, 2004/11/12 & 91 & 21 & $6.0 \times 10^{2}$ & 320 & -- & -- & -- & -- & -- & -- & -- \\
\hline Missouri, 2004/02/10 & 96 & 21 & 770 & 810 & -- & -- & -- & -- & -- & -- & -- \\
\hline Missouri, 2004/04/14 & 164 & 30. & 610 & $1.10 \times 10^{3}$ & -- & -- & -- & -- & -- & -- & -- \\
\hline Missouri, 2004/07/12 & 123 & 33 & $6.0 \times 10^{2}$ & 880 & -- & -- & -- & -- & -- & -- & -- \\
\hline Missouri, 2004/11/12 & 57 & 15.6 & 360 & 420 & -- & -- & -- & -- & -- & -- & -- \\
\hline Ohio, 2004/02/12 & 25 & 16.0 & 710 & 540 & -- & -- & -- & -- & -- & -- & -- \\
\hline Ohio, 2004/04/14 & 34 & 17.0 & 590 & 520 & -- & -- & -- & -- & -- & -- & -- \\
\hline Ohio, 2004/07/17 & 51 & 35 & 480 & $5.0 \times 10^{2}$ & -- & -- & -- & -- & -- & -- & -- \\
\hline Ohio, 2004/11/13 & 43 & 21 & 500 & 560 & -- & -- & -- & -- & -- & -- & -- \\
\hline
\end{tabular}




\begin{tabular}{|c|c|c|c|c|c|c|c|c|c|c|c|c|}
\hline B & $\begin{array}{l}\text { River, date } \\
\qquad(\mathrm{yr} / \mathrm{m} / \mathrm{d})\end{array}$ & $\begin{array}{c}\text { Re, } \\
\text { pmol kg-1 }\end{array}$ & $\begin{array}{c}\text { Mo, } \\
\text { nmol kg-1 }\end{array}$ & $\begin{array}{c}\mathrm{Cl}, \\
\mu \mathrm{mol} \mathrm{kg}-1\end{array}$ & $\begin{array}{c}\mathrm{SO}_{4} \\
\mu \mathrm{mol} \mathrm{kg}\end{array}$ & $\begin{array}{c}\mathrm{Na}, \\
\mu \mathrm{mol} \mathrm{kg}\end{array}$ & $\begin{array}{c}\mathrm{Mg}, \\
\mu \mathrm{mol} \mathrm{kg}{ }^{-1}\end{array}$ & $\begin{array}{c}\mathrm{Ca}, \\
\mu \mathrm{mol} \mathrm{kg}-1\end{array}$ & $\begin{array}{c}\mathrm{K}, \\
\mu \mathrm{mol} \mathrm{kg}\end{array}$ & $\begin{array}{c}\mathrm{Rb}, \\
\mathrm{nmol} \mathrm{kg}\end{array}$ & $\begin{array}{c}\mathrm{Sr}, \\
\mathrm{nmol} \mathrm{kg}\end{array}$ & $\begin{array}{c}\mathrm{Ba}, \\
\mathrm{nmol} \mathrm{kg}^{-1}\end{array}$ \\
\hline & Arkansas, 2004/02/12 & 19 & 6.6 & 1,110 & 280 & -- & -- & -- & -- & -- & -- & -- \\
\hline & Arkansas, 2004/04/15 & 27 & 9.3 & 1,390 & 370 & -- & -- & -- & -- & -- & -- & -- \\
\hline & Arkansas, 2004/07/17 & 22 & 9.3 & 1,350 & 320 & -- & -- & -- & -- & -- & -- & -- \\
\hline & Arkansas, 2004/11/13 & 17.7 & 12.4 & 1,520 & 310 & -- & -- & -- & -- & -- & -- & -- \\
\hline
\end{tabular}

Table 2.1: B: River time-series samples, sampling dates, and chemical data for Arctic rivers and Mississippi tributaries. Chemical data are listed to the last significant digit. Where the last significant digit is zero, this is indicated by a decimal point or scientific notation. Uncertainties are as listed in Section 2.3. 


\begin{tabular}{|c|c|c|c|c|c|c|c|}
\hline River & $\begin{array}{l}\text { Graham } \\
\text { Region }\end{array}$ & Continent & $\begin{array}{c}\mathrm{H}_{2} \mathrm{O} \\
\mathrm{km}^{3} \mathrm{yr}^{-1}\end{array}$ & $\begin{array}{l}\text { Drainage } \\
\text { area, } \mathrm{km}^{2}\end{array}$ & $\begin{array}{l}\text { Sediment, } \\
\text { Pg yr }^{-1}\end{array}$ & $\begin{array}{l}\text { Latitude, } \\
\text { decimal }\end{array}$ & $\begin{array}{r}\text { Longitude, } \\
\text { decimal }\end{array}$ \\
\hline Lena & 1 & Asia & 525 & $2,439,000$ & 17.8 & 66.7664 & 123.3967 \\
\hline $\mathrm{Ob}$ & 1 & Asia & 404 & $2,570,130$ & 16 & 66.5414 & 66.4722 \\
\hline Kolyma & 1 & Asia & 132 & 660,000 & 14.1 & 66.5414 & 2.6458 \\
\hline Yenisei & 1 & Asia & 620 & $2,580,000$ & 9.45 & 67.4344 & 86.3908 \\
\hline Mackenzie & 2 & $\mathrm{~N}$ Amer & 308 & $1,712,738$ & 112.5 & 67.4521 & -133.7389 \\
\hline Saint Lawrence & 3 & N Amer & 447 & $1,100,000$ & 3.8 & 45.8586 & -73.2397 \\
\hline Mississippi & 3 & N Amer & 529 & $3,270,000$ & 450 & 29.9208 & 29.9208 \\
\hline Hudson & 3 & N Amer & 17.3 & 34,000 & 0.6 & 42.7611 & 42.7611 \\
\hline Housatonic & 3 & N Amer & 2.4 & 2,500 & 0.5 & 41.3852 & 41.3852 \\
\hline Connecticut & 3 & $\mathrm{~N}$ Amer & 14.2 & $26,509.5$ & - & 41.4853 & 41.4853 \\
\hline Rhine & 4 & Europe & 69.4 & 185,000 & 2.8 & 50.9481 & 6.9714 \\
\hline Olfusa & 4 & Europe & 13.9 & 6,000 & - & 63.9383 & -21.0083 \\
\hline Thjorsa & 4 & Europe & 12.6 & $6,981.5$ & - & 63.9300 & -20.6400 \\
\hline Amazon & 5 & S Amer & 6590 & $6,133,120.0$ & 1175.0 & 0.0333 & -51.0500 \\
\hline Congo & 6 & Africa & 1200 & $3,710,000$ & 32.8 & -4.2990 & 15.2777 \\
\hline Orange & 6 & Africa & 11.4 & 945,000 & 89 & -28.0833 & 16.8917 \\
\hline Indus & 8 & Asia & 57 & $1,025,866.5$ & 250 & 25.4422 & 68.3164 \\
\hline Meghna & 8 & Asia & 111.0 & $87,500.0$ & - & 23.5993 & 90.6102 \\
\hline Brahmaputra & 8 & Asia & 510 & 595,000 & 630 & 24.4084 & 24.4084 \\
\hline Ganga & 8 & Asia & 493 & $1,033,052$ & 522 & 24.0553 & 89.0314 \\
\hline Yangtze & 9 & Asia & 928 & $1,808,000$ & 490 & 30.2872 & 111.5264 \\
\hline Pearl & 9 & Asia & 7.8 & 17,200 & 0.8 & 26.1153 & 113.2681 \\
\hline Hong & 9 & Asia & 123 & 162,500 & 123 & 21.0544 & 105.8472 \\
\hline Fly & 9 & Oceania & 141 & 64,500 & 110.0 & 0.0000 & 0.0000 \\
\hline Purari & 9 & Oceania & 84.13 & 31,000 & 90 & 0.0000 & 0.0000 \\
\hline Kikori & 9 & Oceania & 40 & 13,200 & - & 0.0000 & 0.0000 \\
\hline Sepik & 9 & Oceania & 120 & 78,350 & 44.08 & 0.0000 & 0.0000 \\
\hline Yukon & 10 & $\mathrm{~N}$ Amer & 200 & 847,642 & 60 & 61.9486 & -162.9077 \\
\hline Copper & 10 & $\mathrm{~N}$ Amer & 34.1 & 62,678 & 70 & 60.4453 & -145.0667 \\
\hline Fraser & 10 & N Amer & 112 & 236,350 & 17.2 & 49.5056 & -121.4142 \\
\hline Andalien & 11 & S Amer & 0.48 & & - & -36.8019 & -73.9667 \\
\hline Bio Bio & 11 & S Amer & 31.69 & 24,782 & - & -36.8088 & -73.0979 \\
\hline Tinguiririca (trib. of Rapel) & 11 & S Amer & 5.38 & 15,157 & - & -34.6125 & -70.9818 \\
\hline Tolten & 11 & S Amer & 18.4 & 8,040 & - & -39.0109 & -73.0818 \\
\hline Itata & 11 & S Amer & 11.39 & 11,385 & - & -36.6242 & -36.6242 \\
\hline Maipo & 11 & S Amer & 3.14 & 15,157 & - & -33.6288 & -70.3548 \\
\hline Maule & 11 & S Amer & 17.96 & 20,865 & - & -35.7236 & -71.1763 \\
\hline Danube & 16 & Europe & 207 & 802,843 & 68 & 47.5000 & 19.0500 \\
\hline
\end{tabular}

Table 2.2: A: List of exorheic rivers, basin characteristics, and sampling locations. Graham regions are 19 subdivisions of the total continental area delineated according to runoff into specific regions of the world ocean and large inland seas (GRAHAM ET AL., 1999). 


\begin{tabular}{|c|c|c|c|c|c|c|c|c|c|c|c|}
\hline $\begin{array}{l}\text { B } \\
\text { River }\end{array}$ & $\begin{array}{c}\operatorname{Re} \\
\text { pmol kg }\end{array}$ & $\begin{array}{c}\text { Mo } \\
\text { nmol kg }\end{array}$ & $\underset{\mu \mathrm{mol} \mathrm{kg}}{\mathrm{Cl}}$ & $\underset{\mu \mathrm{mol} \mathrm{kg}}{\mathrm{SO}_{4}}$ & $\underset{\mu \mathrm{mol} \mathrm{kg}}{\mathrm{Na}}$ & $\underset{\mu \mathrm{mol} \mathrm{kg}}{\mathrm{Mg}}$ & $\begin{array}{c}\mathrm{Ca} \\
\mu \mathrm{mol} \mathrm{kg}\end{array}$ & $\underset{\mu \mathrm{mol} \mathrm{kg}}{\mathrm{K}}$ & $\begin{array}{c}\mathrm{Rb} \\
\mathrm{nmol} \mathrm{kg}\end{array}$ & $\begin{array}{c}\mathrm{Sr} \\
\mathrm{nmol} \mathrm{kg}\end{array}$ & $\begin{array}{c}\mathrm{Ba} \\
\mathrm{nmol} \mathrm{kg}{ }^{1}\end{array}$ \\
\hline Lena & 2.9 & 3.0 & 260 & 96 & 370 & 178 & 380 & 16 & 5.3 & 800 & 78 \\
\hline $\mathrm{Ob}$ & 12.5 & 3.9 & 135 & 83 & $3.0 \times 10^{2}$ & 185 & 460 & 28 & 4.7 & $5.0 \times 10^{2}$ & 66 \\
\hline Kolyma & 2.9 & 1.47 & 92 & 100. & 55 & 870 & 250 & 15 & b.d. & 230 & 42 \\
\hline Yenisei & 6.5 & 3.5 & 168 & 73 & $2.0 \times 10^{2}$ & 131 & 360 & 10 & 1.0 & 600 & 49 \\
\hline Mackenzie & 16.2 & 10.1 & 240 & 380 & 350 & 358 & 870 & 23 & 7 & 1,300 & 280 \\
\hline Saint Lawrence & 24 & 12.1 & 580 & 230 & 520 & 292 & 810 & 35 & 10. & 1,200 & 109 \\
\hline Mississippi & 57 & 21 & 640 & 510 & - & - & - & - & - & - & - \\
\hline Hudson & 7.2 & 3.2 & 660 & 125 & - & - & - & - & - & - & - \\
\hline Housatonic & 6.7 & 5.5 & - & - & - & - & - & - & - & - & - \\
\hline Connecticut & 14.4 & 7.8 & - & - & - & - & - & - & - & - & - \\
\hline Rhine & 57 & 10.8 & 1,210 & $4.0 \times 10^{2}$ & 800 & 356 & $1.5 \times 10^{3}$ & 78 & 24 & 2,600 & 190 \\
\hline Olfusa & 1.74 & 1.04 & 153 & 24 & $3.0 \times 10^{2}$ & 565 & $1.0 \times 10^{2}$ & 12 & 2.0 & b.d. & 3,300 \\
\hline Thjorsa & 4.1 & 4.1 & 86 & 61 & 320 & 631 & $1.0 \times 10^{2}$ & 11 & b.d. & b.d. & 13 \\
\hline Amazon & 1.8 & 0.89 & 8,800 & 92 & 10. & 678 & 170 & 30. & 40. & 150 & 240 \\
\hline Congo & 3.0 & 0.45 & 36 & 18 & 9 & 621 & 53 & 39 & 31 & 17 & 79 \\
\hline Orange & 37 & 24 & 2,800 & 1,210 & 3,700 & 1,090 & $1.0 \times 10^{3}$ & 64 & 1.9 & $2.0 \times 10^{3}$ & 330 \\
\hline Indus & 29 & 36 & 840 & 57 & 530 & 472 & $1.1 \times 10^{3}$ & 110 & 7 & 5,700 & $3.0 \times 10^{2}$ \\
\hline Meghna & 1.40 & 2.4 & 80. & 50. & 240 & 146 & 240 & 31 & 17 & 170 & 45 \\
\hline Brahmaputra & 4.4 & 11.1 & 43 & 156 & 180 & 217 & 620 & 58 & 27 & 500 & 89 \\
\hline Ganga & 3.9 & 10.7 & 67 & 79 & 220 & 194 & 620 & 79 & 14 & 450 & 140 \\
\hline Yangtze & 55 & 15.8 & 310 & 420 & 440 & 373 & 700 & 47 & 15 & 1,700 & 240 \\
\hline Pearl & 10.9 & 12.3 & 220 & 174 & 320 & 65.8 & 410 & 84 & 110 & 170 & 90 \\
\hline Hong & 13.3 & 6.7 & 68 & 126 & 170 & 218 & 750 & 44 & 28 & 1,100 & 190 \\
\hline Fly & 53 & 59 & 15,000 & 190 & 210 & 118 & 870 & 20. & 5.6 & 90 & 70. \\
\hline Purari & 3.4 & 4.0 & 14,000 & 87 & 750 & 278 & 710 & 40 & 13 & 70 & 53 \\
\hline Kikori & 9.3 & 3.5 & 12,000 & 17 & 5.5 & 281 & $1.1 \times 10^{3}$ & 9 & 5.4 & 90 & 37 \\
\hline Sepik & 1.64 & 2.0 & - & & 610 & 243 & 250 & 24 & 14 & 18 & 89 \\
\hline Yukon & 13.4 & 12.0 & 65 & 280 & 120 & 297 & 790 & 38 & 21 & 60 & 230 \\
\hline Copper & 9.3 & 14.4 & 53 & 220 & 110 & 107 & 520 & 36 & 11 & 22 & 85 \\
\hline Fraser & 5.3 & 7.9 & 21 & 98 & 9 & 126 & 410 & 16 & 10. & 50 & 57 \\
\hline Andalien & 7.2 & 1.26 & 153 & 6.2 & 510 & 162 & 320 & 36 & 15 & 80 & 57 \\
\hline Bio Bio & 4.9 & 2.3 & 95 & 47 & 220 & 100. & 190 & 26 & 24 & 27 & 18 \\
\hline Tinguiririca (trib. of Rapel) & 20. & 14.4 & 139 & 460 & 280 & 112 & 510 & 28 & 41 & 70 & 23 \\
\hline Tolten & 1.88 & 4.6 & 41 & 21 & 170 & 65.7 & 110 & 22 & 21 & 16 & 17 \\
\hline Itata & 1.14 & 4.7 & 190 & 16.3 & 550 & 283 & 280 & 34 & 14 & 90 & 73 \\
\hline Maipo & 53 & 33 & 2,500 & 3,300 & $3.0 \times 10^{2}$ & 387 & $3.7 \times 10^{3}$ & 70. & 70 & $1.0 \times 10^{4}$ & 86 \\
\hline Maule & 7.4 & 19 & 190 & 1,740 & 370 & 129 & 290 & 33 & 46 & 44 & 22 \\
\hline Danube & 74 & 10.8 & 1,830 & 1,030 & $2.0 \times 10^{3}$ & $1.20 \times 10^{3}$ & $1.9 \times 10^{3}$ & 160 & 32 & 3,300 & $4.0 \times 10^{2}$ \\
\hline
\end{tabular}

Table 2.2: B: List of exorheic rivers and chemical data used to re-evaluate Mo and Re world river averages. Chemical data are listed to the last significant digit. Where the last significant digit is zero, this is indicated by a decimal point or scientific notation. Uncertainties are as listed in Section 2.3 


\begin{tabular}{|c|c|c|c|c|c|c|}
\hline \multicolumn{7}{|l|}{ A } \\
\hline River & $\begin{array}{c}\text { Date } \\
\text { yyyy/mm/dd }\end{array}$ & $\begin{array}{l}\text { Graham } \\
\text { Region }\end{array}$ & Continent & Country & $\begin{array}{l}\text { Latitude, } \\
\text { decimal }\end{array}$ & $\begin{array}{c}\text { Longitude, } \\
\text { decimal }\end{array}$ \\
\hline Lena & 2004 & 1 & Asia & Russia & 66.7664 & 123.3967 \\
\hline $\mathrm{Ob}$ & 2004 & 1 & Asia & Russia & 66.5414 & 66.4722 \\
\hline Kolyma & 2004 & 1 & Asia & Russia & 68.7500 & 2.6458 \\
\hline Yenisei & 2004 & 1 & Asia & Russia & 67.4344 & 86.3908 \\
\hline Ob (Yamal Nemetz) & 2006/11/09 & 1 & Asia & Russia & 71.4833 & 71.8000 \\
\hline Mackenzie & 2004 & 2 & N Amer & Canada & 67.4521 & -133.7389 \\
\hline Mackenzie & $2007 / 07 / 04$ & 2 & N Amer & Canada & 68.4659 & -134.1283 \\
\hline Saint Lawrence (Coteau du Lac) & $2008 / 05 / 18$ & 3 & N Amer & Canada & 45.2798 & -74.1782 \\
\hline Saint Lawrence (Contrecoeur) & $2008 / 05 / 18$ & 3 & N Amer & Canada & 45.8586 & -73.2397 \\
\hline Mississippi & 2004 & 3 & N Amer & U. S. A. & 29.9208 & -90.1353 \\
\hline Missourri & $2005 / 05 / 18$ & 3 & $\mathrm{~N}$ Amer & U. S. A. & 41.3569 & -95.9502 \\
\hline Platte & $2005 / 05 / 18$ & 3 & N Amer & U. S. A. & 41.0148 & -96.1580 \\
\hline Platte, North Channel & $2005 / 05 / 18$ & 3 & N Amer & U. S. A. & 41.0194 & -100.3715 \\
\hline Platte, South Channel & $2005 / 05 / 18$ & 3 & N Amer & U. S. A. & 41.0545 & -102.0732 \\
\hline North Platte & $2005 / 05 / 18$ & 3 & N Amer & U. S. A. & 41.3171 & -102.1262 \\
\hline Powder, North Fork & $2005 / 05 / 19$ & 3 & N Amer & U. S. A. & 43.7726 & -106.7103 \\
\hline Powder, South Fork & $2005 / 05 / 19$ & 3 & $\mathrm{~N}$ Amer & U. S. A. & 43.7084 & -106.6036 \\
\hline Bighorn & $2005 / 05 / 19$ & 3 & N Amer & U. S. A. & 45.6446 & -107.6585 \\
\hline Yellowstone & $2005 / 05 / 19$ & 3 & N Amer & U. S. A. & 45.5977 & -110.5658 \\
\hline Missouri & $2005 / 05 / 19$ & 3 & N Amer & U. S. A. & 45.9399 & -111.4904 \\
\hline Hot Spring & $2005 / 05 / 20$ & 3 & N Amer & U. S. A. & 46.1372 & -112.8918 \\
\hline Blackfoot & $2005 / 05 / 20$ & 3 & N Amer & U. S. A. & 46.8737 & -113.8855 \\
\hline Bitterroot & $2005 / 05 / 20$ & 3 & N Amer & U. S. A. & 46.6323 & -114.0663 \\
\hline Silver Bow Creek & $2005 / 05 / 25$ & 3 & N Amer & U. S. A. & 45.9957 & -112.5388 \\
\hline Madison & $2005 / 05 / 25$ & 3 & N Amer & U. S. A. & 45.9009 & -111.5261 \\
\hline Gallatin & $2005 / 05 / 25$ & 3 & N Amer & U. S. A. & 45.9342 & -111.4931 \\
\hline Jefferson & $2005 / 05 / 25$ & 3 & $\mathrm{~N}$ Amer & U. S. A. & 45.8973 & -111.6104 \\
\hline Yellowstone & $2005 / 05 / 25$ & 3 & N Amer & U. S. A. & 47.2814 & -104.5248 \\
\hline Missouri & $2005 / 05 / 25$ & 3 & N Amer & U. S. A. & 46.7591 & -100.8410 \\
\hline Mississippi & $2005 / 05 / 27$ & 3 & N Amer & U. S. A. & 45.3257 & -93.8239 \\
\hline St. Croix River & $2005 / 05 / 27$ & 3 & N Amer & U. S. A. & 44.9613 & -92.7737 \\
\hline Mississippi (New Orleans) & $2004 / 04$ & 3 & N Amer & U. S. A. & 29.9566 & -90.0615 \\
\hline South Platte River (11 Mile Canyon) & $2008 / 16 / 17$ & 3 & N Amer & U. S. A. & 38.9268 & -105.4251 \\
\hline Clay City stream & $2000 / 01 / 12$ & 3 & N Amer & U. S. A. & 37.8733 & -83.9478 \\
\hline
\end{tabular}




\begin{tabular}{|c|c|c|c|c|c|c|}
\hline \multicolumn{7}{|l|}{$\mathbf{A}$} \\
\hline River & $\begin{array}{c}\text { Date } \\
\text { yyyy } / \mathrm{mm} / \mathrm{dd}\end{array}$ & $\begin{array}{l}\text { Graham } \\
\text { Region }\end{array}$ & Continent & Country & $\begin{array}{l}\text { Latitude, } \\
\text { decimal }\end{array}$ & $\begin{array}{c}\text { Longitude, } \\
\text { decimal }\end{array}$ \\
\hline Hudson & 2006/06/06 & 3 & N Amer & U. S. A. & 42.7611 & -37.6847 \\
\hline Croton Outlet & $2004 / 09 / 29$ & 3 & N Amer & U. S. A. & 41.2069 & -73.8217 \\
\hline Croton Outlet & $2004 / 09 / 29$ & 3 & N Amer & U. S. A. & 41.2069 & -73.8217 \\
\hline Hudson & $2004 / 09 / 30$ & 3 & $\mathrm{~N}$ Amer & U. S. A. & 41.8321 & -73.9415 \\
\hline Hudson & $2004 / 09 / 30$ & 3 & $\mathrm{~N}$ Amer & U. S. A. & 41.8321 & -73.9415 \\
\hline Pond in Hudson River watershed & $2004 / 09 / 30$ & 3 & N Amer & U. S. A. & 41.9240 & -73.9109 \\
\hline Esophus Creek & $2004 / 09 / 30$ & 3 & N Amer & U. S. A. & 42.0677 & -74.3057 \\
\hline Esophus Creek & $2004 / 09 / 30$ & 3 & N Amer & U. S. A. & 42.0677 & -74.3057 \\
\hline Shoharie Creek & $2004 / 09 / 30$ & 3 & $\mathrm{~N}$ Amer & U. S. A. & 42.5999 & -74.3360 \\
\hline Shoharie Creek & $2004 / 09 / 30$ & 3 & N Amer & U. S. A. & 42.5999 & -74.3360 \\
\hline Mohawk & $2004 / 09 / 30$ & 3 & $\mathrm{~N}$ Amer & U. S. A. & 42.8484 & -74.0143 \\
\hline Lake George & $2004 / 10 / 01$ & 3 & N Amer & U. S. A. & 43.4200 & -73.7086 \\
\hline Schaeffer's Creek & $2004 / 10 / 01$ & 3 & N Amer & U. S. A. & 43.2861 & -73.8217 \\
\hline Upper Hudson & $2004 / 10 / 01$ & 3 & N Amer & U. S. A. & 43.2873 & -73.8262 \\
\hline Upper Hudson & $2004 / 10 / 01$ & 3 & N Amer & U. S. A. & 43.2873 & -73.8262 \\
\hline Housatonic & $2004 / 07 / 08$ & 3 & N Amer & U. S. A. & 41.3852 & -73.1724 \\
\hline Housatonic & $2004 / 07 / 08$ & 3 & N Amer & U. S. A. & & \\
\hline Connecticut & $2004 / 07 / 08$ & 3 & N Amer & U. S. A. & 41.4853 & -72.5142 \\
\hline Connecticut & $2004 / 07 / 08$ & 3 & N Amer & U. S. A. & & \\
\hline Connecticut & $2004 / 07 / 08$ & 3 & N Amer & U. S. A. & 41.4816 & -72.5066 \\
\hline Connecticut & $2004 / 07 / 08$ & 3 & N Amer & U. S. A. & & \\
\hline Coffee Creek & $2000 / 08 / 26$ & 3 & N Amer & U. S. A. & 41.1235 & -122.8203 \\
\hline Upper Cabin Meadow Lake & $2000 / 08 / 25$ & 3 & N Amer & U. S. A. & 41.3398 & -122.5884 \\
\hline Runoff from Josephine Peridotite & $2000 / 08 / 23$ & 3 & N Amer & U. S. A. & 42.1836 & -123.9933 \\
\hline Big Vulcan Lake & $2000 / 08 / 23$ & 3 & $\mathrm{~N}$ Amer & U. S. A. & 42.1877 & -123.9845 \\
\hline Pine Creek & 2000/??/?? & 3 & N Amer & U. S. A. & 43.0349 & -109.7648 \\
\hline Willow Lake & $2000 / ? ? / ? ?$ & 3 & N Amer & U. S. A. & 42.9911 & -109.8993 \\
\hline Freemont Lake & $2000 / ? ? / ? ?$ & 3 & N Amer & U. S. A. & & \\
\hline Half Moon Lake & $2000 / ? ? / ? ?$ & 3 & N Amer & U. S. A. & 42.9365 & -109.7620 \\
\hline Freemont Lake & $2000 / ? ? / ? ?$ & 3 & N Amer & U. S. A. & 42.9453 & -109.7951 \\
\hline Soda Lake & $2000 / ? ? / ? ?$ & 3 & N Amer & U. S. A. & 42.9558 & -109.8528 \\
\hline Boulder Lake & $2000 / ? ? / ? ?$ & 3 & N Amer & U. S. A. & 42.8558 & -109.6228 \\
\hline Rhine & $2007 / 08 / 25$ & 4 & Europe & Germany & 50.9481 & 6.9714 \\
\hline River at Bluonos & $2006 / 06 / 02$ & 4 & Europe & Iceland & 65.6582 & -20.2855 \\
\hline
\end{tabular}




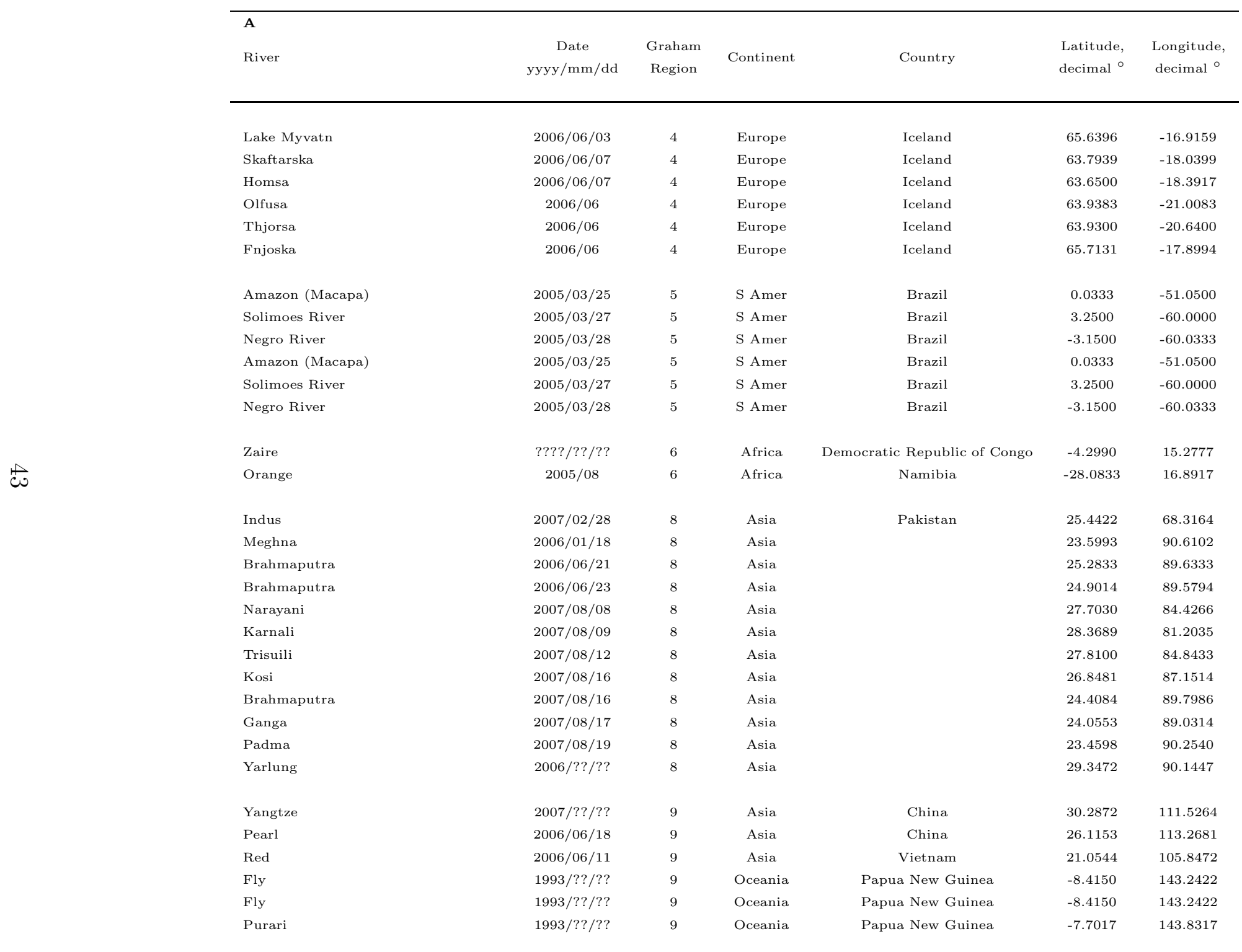




\begin{tabular}{|c|c|c|c|c|c|c|c|}
\hline & \multicolumn{7}{|l|}{ A } \\
\hline & River & $\begin{array}{c}\text { Date } \\
\text { yyyy } / \mathrm{mm} / \mathrm{dd}\end{array}$ & $\begin{array}{c}\text { Graham } \\
\text { Region }\end{array}$ & Continent & Country & $\begin{array}{l}\text { Latitude, } \\
\text { decimal }\end{array}$ & $\begin{array}{c}\text { Longitude, } \\
\text { decimal }\end{array}$ \\
\hline \multirow{34}{*}{$\stackrel{A}{A}$} & Purari & 1993/??/?? & 9 & Oceania & Papua New Guinea & -7.7017 & 143.8317 \\
\hline & Kikori & 1993/??/?? & 9 & Oceania & Papua New Guinea & -7.6809 & 144.8354 \\
\hline & Kikori & 1993/??/?? & 9 & Oceania & Papua New Guinea & -7.6809 & 144.8354 \\
\hline & Fly & 1993/??/?? & 9 & Oceania & Papua New Guinea & -8.4150 & 143.2422 \\
\hline & Fly & 1993/??/?? & 9 & Oceania & Papua New Guinea & -8.4150 & 143.2422 \\
\hline & Sepik & 1993/??/?? & 9 & Oceania & Papua New Guinea & -3.9051 & 144.5403 \\
\hline & Waiahole Stream & $2006 / 08 / 24$ & 9 & Oceania & U. S. A. & 21.4816 & -157.8487 \\
\hline & Waimea Falls Pond & $2006 / 08 / 24$ & 9 & Oceania & U. S. A. & 21.6306 & -158.0440 \\
\hline & Waiamea Reservoir & $2006 / 08 / 24$ & 9 & Oceania & U. S. A. & 21.4908 & -158.0260 \\
\hline & Yukon & 2004 & 10 & N Amer & U. S. A. & 61.9486 & -162.9077 \\
\hline & Copper & $2008 / 08 / 22$ & 10 & N Amer & U. S. A. & 60.4453 & -145.0667 \\
\hline & Thompson & 2006/09/05 & 10 & N Amer & Canada & 50.3492 & -121.3906 \\
\hline & Nechako & $2006 / 09 / 11$ & 10 & N Amer & Canada & & \\
\hline & Upper Fraser & $2006 / 07 / 15$ & 10 & N Amer & Canada & & \\
\hline & Fraser & 2006/09/19 & 10 & N Amer & Canada & & \\
\hline & Quesnel & $2006 / 09 / 18$ & 10 & N Amer & Canada & 52.9833 & -122.4822 \\
\hline & Harrison & $2006 / 07 / 27$ & 10 & N Amer & Canada & 49.2372 & -121.9633 \\
\hline & Blackwater & $2006 / 09 / 18$ & 10 & N Amer & Canada & 53.2875 & -123.1422 \\
\hline & North Thompson & $2006 / 07 / 14$ & 10 & N Amer & Canada & & \\
\hline & Nechako & 2006/09/19 & 10 & N Amer & Canada & & \\
\hline & Fraser & $2006 / 08 / 22$ & 10 & N Amer & Canada & 49.5056 & -121.4142 \\
\hline & Fraser & $2006 / 08 / 08$ & 10 & N Amer & Canada & 49.5633 & -121.4028 \\
\hline & Andalien & $2004 / 02 / 12$ & 11 & S Amer & Chile & -36.8019 & -73.9667 \\
\hline & Bio Bio & $2004 / 02 / 12$ & 11 & S Amer & Chile & -36.8088 & -73.0979 \\
\hline & Bio Bio & $2004 / 02 / 12$ & 11 & S Amer & Chile & -36.8688 & -73.0445 \\
\hline & Andalien & $2004 / 02 / 12$ & 11 & S Amer & Chile & -35.6844 & -71.1106 \\
\hline & Tinguiririca & $2006 / 09 / 16$ & 11 & S Amer & Chile & -34.6125 & -70.9818 \\
\hline & Bio Bio & $2006 / 08 / 24$ & 11 & S Amer & Chile & -36.8393 & -73.0514 \\
\hline & Tolten & $2006 / 06 / 25$ & 11 & S Amer & Chile & -39.0109 & -73.0818 \\
\hline & Itata & $2006 / 06 / 28$ & 11 & S Amer & Chile & -36.6242 & -72.4816 \\
\hline & Maipo & $2007 / 01 / 29$ & 11 & S Amer & Chile & -33.6288 & -70.3548 \\
\hline & Tinguiririca & $2007 / 01 / 30$ & 11 & S Amer & Chile & -34.6789 & -70.8739 \\
\hline & Maule & $2007 / 01 / 31$ & 11 & S Amer & Chile & -35.7236 & -71.1763 \\
\hline & Bio Bio & $2007 / 02 / 01$ & 11 & S Amer & Chile & -37.5991 & -72.2781 \\
\hline
\end{tabular}




\begin{tabular}{|c|c|c|c|c|c|c|}
\hline \multicolumn{7}{|l|}{ A } \\
\hline River & $\begin{array}{c}\text { Date } \\
\text { yyyy } / \mathrm{mm} / \mathrm{dd}\end{array}$ & $\begin{array}{c}\text { Graham } \\
\text { Region }\end{array}$ & Continent & Country & $\begin{array}{l}\text { Latitude, } \\
\text { decimal }\end{array}$ & $\begin{array}{c}\text { Longitude, } \\
\text { decimal }\end{array}$ \\
\hline Itata & $2007 / 02 / 02$ & 11 & S Amer & Chile & -36.4666 & -72.6916 \\
\hline Tolten & $2007 / 02 / 03$ & 11 & S Amer & Chile & -38.9772 & -72.6364 \\
\hline Tolten & $2007 / 02 / 04$ & 11 & S Amer & Chile & -39.2743 & -72.2301 \\
\hline Thermal baths & $2007 / 02 / 06$ & 11 & S Amer & Chile & -41.9749 & -72.5530 \\
\hline glacial river to Huinay & $2007 / 02 / 06$ & 11 & S Amer & Chile & -42.3811 & -72.4155 \\
\hline Pelorus & $2006 / 12 / 20$ & 12 & Oceania & New Zealand & -41.2988 & 173.5734 \\
\hline Danube at Regensburg & $2007 / 05 / 02$ & 16 & Europe & Germany & 49.0214 & 12.1219 \\
\hline March at Angern & $2007 / 05 / 03$ & 16 & Europe & Austria & & \\
\hline Danube at Passau & $2007 / 05 / 02$ & 16 & Europe & Germany & 48.5767 & 13.4567 \\
\hline Danube at Ulm & $2007 / 05 / 02$ & 16 & Europe & Germany & 48.3950 & 9.9928 \\
\hline Regen at Regensburg & $2007 / 05 / 02$ & 16 & Europe & Germany & 49.02114 & 12.1219 \\
\hline Tisza at Szeged & $2007 / 05 / 04$ & 16 & Europe & Hungary & 46.2494 & 20.1533 \\
\hline Danube at Vienna & $2007 / 05 / 04$ & 16 & Europe & Austria & 48.2261 & 16.4086 \\
\hline Inn at Schärding & $2007 / 05 / 02$ & 16 & Europe & Germany & 48.4572 & 13.4267 \\
\hline Iller at Wiblinger & $2007 / 05 / 02$ & 16 & Europe & Germany & & \\
\hline Duna at Budapest & $2007 / 05 / 04$ & 16 & Europe & Hungary & 47.5000 & 19.0500 \\
\hline
\end{tabular}

Table 2.3: A: Sample, sampling date and location information for tributary and exorheic river samples. 


\begin{tabular}{|c|c|c|c|c|c|c|c|c|c|c|c|}
\hline River & $\begin{array}{c}\mathrm{Re}, \\
\mathrm{pmol} \mathrm{kg}^{-1}\end{array}$ & $\begin{array}{c}\text { Mo, } \\
\mathrm{nmol} \mathrm{kg}^{-1}\end{array}$ & $\begin{array}{c}\mathrm{Cl}, \\
\mu \mathrm{mol} \mathrm{\textrm {kg } ^ { - }} 1\end{array}$ & $\begin{array}{c}\mathrm{SO}_{4}, \\
\mu \mathrm{mol} \mathrm{kg}{ }^{-} 1\end{array}$ & $\begin{array}{c}\mathrm{Na} \\
\mu \mathrm{mol} \mathrm{kg}^{-} 1\end{array}$ & $\begin{array}{c}\mathrm{Mg} \\
\mu \mathrm{mol} \mathrm{kg}\end{array}$ & $\begin{array}{c}\mathrm{Ca}, \\
\mu \mathrm{mol} \mathrm{kg}{ }^{-} 1\end{array}$ & $\begin{array}{c}\mathrm{K}, \\
\mu \mathrm{mol} \mathrm{kg}\end{array}$ & $\begin{array}{c}\mathrm{Rb} \\
\mathrm{nmol} \mathrm{kg}\end{array}$ & $\begin{array}{c}\mathrm{Sr} \\
\mathrm{nmol} \mathrm{kg}\end{array}$ & $\begin{array}{c}\mathrm{Ba}, \\
\mathrm{nmol} \mathrm{kg}\end{array}$ \\
\hline Lena & 2.91 & 2.96 & 258 & 96 & 372.2 & 178.2 & 380.6 & 15.6 & 5.27 & 816.0 & 77.5 \\
\hline $\mathrm{Ob}$ & 12.45 & 3.85 & 135 & 83 & 295.9 & 184.6 & 462.5 & 27.7 & 4.71 & 497.8 & 66.4 \\
\hline Kolyma & 2.89 & 1.47 & 92 & 100 & 54.8 & 86.8 & 249.4 & 15.1 & b.d. & 228.6 & 42.1 \\
\hline Yenisei & 6.46 & 3.49 & 168 & 73 & 203.5 & 131.4 & 362.8 & 10.2 & 1.04 & 643.6 & 48.7 \\
\hline Ob (Yamal Nemetz) & 7.64 & 2.77 & 1773 & 625 & 230.3 & 185.1 & 431.1 & 23.5 & 3.57 & 428.8 & 84.4 \\
\hline Mackenzie & 16.15 & 10.08 & 240 & 378 & 352.9 & 358.5 & 870.8 & 22.9 & 7.35 & 1321.7 & 274.9 \\
\hline Mackenzie & 14.10 & 11.10 & 440 & 439 & 71.0 & 413.5 & 1009.6 & 3.5 & 0.67 & 2131.3 & 275.1 \\
\hline Saint Lawrence (Coteau du Lac) & 17.76 & 12.34 & 607 & 236 & 517.2 & 299.2 & 850.0 & 36.2 & 9.02 & 1217.8 & 106.4 \\
\hline Saint Lawrence (Contrecoeur) & 23.88 & 12.05 & 581 & 234 & 517.6 & 292.4 & 807.4 & 35.3 & 9.86 & 1164.4 & 109.0 \\
\hline Mississippi & 57.00 & 21.00 & 642 & 509 & 0.0 & 0.0 & 0.0 & 0.0 & 0.00 & 0.0 & 0.0 \\
\hline Missouri & 330.27 & 38.58 & 490 & 1813 & 2126.2 & 1142.7 & 1631.6 & 137.8 & 19.85 & 3610.7 & 309.0 \\
\hline Platte & 217.34 & 35.84 & 275 & 0 & 889.4 & 632.9 & 1596.0 & 246.6 & 20.79 & 2351.3 & 731.7 \\
\hline Platte, North Channel & 44.11 & 29.72 & 77 & 147 & 614.5 & 276.1 & 1388.4 & 238.6 & 48.93 & 1907.3 & 975.9 \\
\hline Platte, South Channel & 1244.60 & 34.11 & 3046 & 8970 & 8804.1 & 2464.1 & 3905.9 & 483.2 & 61.96 & 13715.3 & 185.4 \\
\hline North Platte & 304.24 & 26.90 & 368 & 1093 & 2399.2 & 482.6 & 930.5 & 227.5 & 60.62 & 3025.5 & 600.0 \\
\hline Powder, North Fork & 87.00 & 8.76 & 102 & 1783 & 1827.6 & 962.1 & 1551.9 & 55.0 & 7.92 & 4207.5 & 221.1 \\
\hline Powder, South Fork & 16.84 & 7.61 & 246 & 767 & 645.3 & 472.3 & 1004.0 & 39.3 & 5.00 & 2528.8 & 100.5 \\
\hline Bighorn & 190.79 & 28.35 & 425 & 3739 & 4706.3 & 1348.8 & 1373.4 & 135.7 & 30.51 & 7931.5 & 242.0 \\
\hline Yellowstone & 4.11 & 7.03 & 75 & 97 & 307.3 & 135.8 & 279.6 & 51.6 & 63.67 & 215.0 & 79.5 \\
\hline Missouri & 14.86 & 14.04 & 110 & 221 & 400.9 & 363.8 & 867.7 & 59.0 & 26.36 & 823.9 & 196.2 \\
\hline Hot Spring & 8.21 & 33.31 & 13 & 79 & 85.5 & 174.3 & 613.1 & 25.0 & 12.94 & 166.0 & 83.5 \\
\hline Blackfoot & 2.90 & 2.94 & 15 & 40 & 7.1 & 285.5 & 580.3 & 1.7 & 0.28 & 164.5 & 620.1 \\
\hline Bitterroot & 1.23 & 3.82 & 14 & 18 & 88.6 & 41.6 & 142.5 & 22.5 & 3.48 & 68.1 & 59.5 \\
\hline Silver Bow Creek & 39.93 & 81.50 & 245 & 0 & 389.2 & 262.1 & 709.7 & 60.8 & 7.15 & 1138.2 & 161.7 \\
\hline Madison & 10.22 & 52.40 & 513 & 126 & 1469.6 & 196.5 & 497.2 & 104.0 & 191.05 & 301.0 & 107.2 \\
\hline Gallatin & 11.33 & 7.55 & 51 & 159 & 226.1 & 300.3 & 732.1 & 44.5 & 9.81 & 675.1 & 180.9 \\
\hline Jefferson & 17.83 & 23.02 & 91 & 268 & 341.7 & 290.6 & 642.5 & 55.3 & 10.60 & 542.4 & 172.7 \\
\hline Yellowstone & 29.26 & 11.34 & 94 & 557 & 712.4 & 339.2 & 651.3 & 46.5 & 11.92 & 867.7 & 145.3 \\
\hline Missouri & 93.41 & 32.41 & 324 & 1844 & 2868.1 & 859.0 & 1194.4 & 99.1 & 18.09 & 2868.7 & 267.6 \\
\hline Mississippi & 13.54 & 8.16 & 248 & 96 & 199.4 & 521.5 & 1042.2 & 35.8 & 8.74 & 175.8 & 234.8 \\
\hline St. Croix River & 8.61 & 3.54 & 157 & 44 & 122.8 & 306.0 & 554.9 & 24.2 & 4.46 & 25.8 & 80.6 \\
\hline Mississippi (New Orleans) & 29.01 & 13.91 & 761 & 461 & 659.5 & 478.3 & 975.5 & 47.1 & 2.28 & 574.4 & 258.0 \\
\hline South Platte River (11 Mile Canyon) & 37.38 & 19.12 & 1219 & 578 & 1496.0 & 611.3 & 992.4 & 50.8 & 7.18 & 2570.9 & 187.6 \\
\hline Clay City stream & 157.30 & 11.67 & 660 & 3520 & 200.4 & 395.0 & 1036.7 & 105.2 & 28.85 & 529.6 & 92.6 \\
\hline
\end{tabular}




\begin{tabular}{|c|c|c|c|c|c|c|c|c|c|c|c|}
\hline River & $\begin{array}{c}\text { Re } \\
\text { pmol kg }^{-1}\end{array}$ & $\begin{array}{c}\text { Mo, } \\
\text { nmol kg }^{-1}\end{array}$ & $\begin{array}{c}\mathrm{Cl}, \\
\mu \mathrm{mol} \mathrm{kg}{ }^{-} 1\end{array}$ & $\begin{array}{c}\mathrm{SO}_{4}, \\
\mu \mathrm{mol} \mathrm{kg}{ }^{-} 1\end{array}$ & $\begin{array}{c}\mathrm{Na}, \\
\mu \mathrm{mol} \mathrm{kg}{ }^{-} 1\end{array}$ & $\begin{array}{c}\mathrm{Mg}, \\
\mu \mathrm{mol} \mathrm{kg}{ }^{-} 1\end{array}$ & $\begin{array}{c}\mathrm{Ca} \\
\mu \mathrm{mol} \mathrm{kg}{ }^{-} 1\end{array}$ & $\begin{array}{c}\mathrm{K}, \\
\mu \mathrm{mol} \mathrm{kg}{ }^{-} 1\end{array}$ & $\begin{array}{c}\mathrm{Rb}, \\
\mathrm{nmol} \mathrm{kg}^{-1}\end{array}$ & $\begin{array}{c}\mathrm{Sr}, \\
\mathrm{nmol} \mathrm{kg}^{-1}\end{array}$ & $\begin{array}{c}\mathrm{Ba} \\
\mathrm{nmol} \mathrm{kg}^{-1}\end{array}$ \\
\hline Hudson & 7.2 & 3.2 & 660 & 124 & & & & & & & \\
\hline Croton Outlet & 5.72 & 4.76 & 1520 & 108 & 1028.8 & 293.0 & 598.3 & 51.8 & 8.53 & 117.7 & 120.2 \\
\hline Croton Outlet & 5.70 & 4.87 & 1532 & 108 & 1059.0 & 296.4 & 604.1 & 53.0 & 9.57 & 114.2 & 131.1 \\
\hline Hudson & 7.66 & 5.74 & 515 & 126 & 447.6 & 173.3 & 640.9 & 38.1 & 3.77 & 350.5 & 87.7 \\
\hline Hudson & 7.72 & 4.24 & 523 & 127 & 457.8 & 173.5 & 657.0 & 38.5 & 3.27 & 343.1 & 98.6 \\
\hline Pond in Hudson River watershed & 6.22 & 1.47 & 713 & 111 & 594.5 & 133.6 & 873.5 & 33.4 & b.d. & 498.0 & 77.3 \\
\hline Esophus Creek & 3.66 & 0.12 & 66 & 46 & 90.5 & 35.7 & 122.4 & 7.7 & b.d. & b.d. & 47.7 \\
\hline Esophus Creek & 3.68 & 0.14 & 66 & 47 & 87.0 & 36.3 & 122.0 & 7.8 & b.d. & b.d. & 46.7 \\
\hline Shoharie Creek & 4.31 & 0.87 & 180 & 57 & 194.0 & 62.3 & 284.9 & 21.1 & b.d. & 11.9 & 57.5 \\
\hline Shoharie Creek & 4.30 & 0.94 & 181 & 57 & 188.8 & 62.4 & 287.5 & 20.5 & b.d. & 6.7 & 55.6 \\
\hline Mohawk & 7.84 & 4.47 & 502 & 153 & 478.8 & 206.6 & 860.9 & 30.5 & b.d. & 845.7 & 104.0 \\
\hline Lake George & 5.19 & 1.34 & 471 & 80 & 405.7 & 102.4 & 351.2 & 12.7 & b.d. & 37.7 & 35.6 \\
\hline Schaeffer's Creek & 4.67 & 2.57 & 181 & 65 & -37.8 & 370.0 & 523.8 & -2.4 & b.d. & b.d. & b.d. \\
\hline Upper Hudson & 3.86 & 1.24 & 303 & 48 & 237.5 & 54.7 & 210.3 & 7.6 & 2.30 & 22.6 & 37.3 \\
\hline Upper Hudson & 3.89 & 1.02 & 303 & 48 & 239.5 & 54.3 & 207.7 & 7.6 & 2.20 & 17.8 & 45.4 \\
\hline Housatonic & 6.66 & 5.43 & & & & & & & & & \\
\hline Housatonic & 6.63 & 5.59 & & & & & & & & & \\
\hline Connecticut & 14.90 & 7.81 & & & & & & & & & \\
\hline Connecticut & 14.76 & 7.74 & & & & & & & & & \\
\hline Connecticut & 13.88 & 7.85 & & & & & & & & & \\
\hline Connecticut & 13.86 & 7.89 & & & & & & & & & \\
\hline Coffee Creek & 3.39 & 3.26 & 192 & 16 & 178.0 & 443.8 & 544.2 & 4.0 & b.d. & 32.0 & 20.2 \\
\hline Upper Cabin Meadow Lake & 0.29 & -0.03 & 2 & 1 & 4.8 & 341.9 & 9.4 & 0.9 & b.d. & b.d. & 159.9 \\
\hline Runoff from Josephine Peridotite & 0.29 & -0.12 & 34 & 5 & 43.7 & 424.2 & 9.9 & 2.6 & b.d. & b.d. & 24.3 \\
\hline Big Vulcan Lake & 0.13 & -0.10 & 25 & 3 & 33.5 & 333.2 & 9.9 & 0.4 & b.d. & b.d. & 16.9 \\
\hline Pine Creek & 3.56 & 1.44 & 6 & 18 & 23.9 & 11.7 & 74.9 & 10.8 & 2.65 & b.d. & 33.5 \\
\hline Willow Lake & 9.41 & 0.43 & 20 & 23 & 47.4 & 21.3 & 72.6 & 13.0 & 1.64 & 15.4 & 42.0 \\
\hline Freemont Lake & 6.57 & 1.29 & 9 & 15 & 24.8 & 11.9 & 52.5 & 9.2 & 1.90 & b.d. & 25.7 \\
\hline Half Moon Lake & 4.11 & 0.65 & 7 & 13 & 25.4 & 15.1 & 51.6 & 8.0 & 0.84 & b.d. & 27.4 \\
\hline Freemont Lake & 6.56 & 1.17 & 9 & 15 & 24.6 & 11.4 & 50.9 & 9.5 & 2.93 & b.d. & 27.5 \\
\hline Soda Lake & 41.89 & 4.87 & 6313 & & 3084.0 & 6149.9 & 2139.6 & 3211.5 & 127.86 & 12712.2 & 85.7 \\
\hline Boulder Lake & 3.96 & 0.67 & 7 & 13 & 27.8 & 14.9 & 56.1 & 10.1 & 3.14 & b.d. & 28.0 \\
\hline Rhine & 56.94 & 10.82 & 1210 & 395 & 808.0 & 356.1 & 1496.9 & 77.9 & 24.05 & 2580.4 & 186.1 \\
\hline River at Bluonos & 1.39 & 1.32 & 93 & 18 & 150.9 & 89.0 & 109.5 & 10.3 & b.d. & b.d. & 17.8 \\
\hline
\end{tabular}




\begin{tabular}{|c|c|c|c|c|c|c|c|c|c|c|c|}
\hline River & $\begin{array}{c}\text { Re, } \\
\text { pmol kg }\end{array}$ & $\begin{array}{c}\text { Mo, } \\
\mathrm{nmol} \mathrm{kg}^{-1}\end{array}$ & 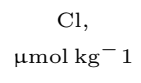 & $\begin{array}{c}\mathrm{SO}_{4} \\
\mu \mathrm{mol} \mathrm{kg}{ }^{-} 1\end{array}$ & $\begin{array}{c}\mathrm{Na} \\
\mu \mathrm{mol} \mathrm{kg}-1\end{array}$ & $\begin{array}{c}\mathrm{Mg}, \\
\mu \mathrm{mol} \mathrm{kg}{ }^{-} 1\end{array}$ & $\begin{array}{c}\mathrm{Ca} \\
\mu \mathrm{mol} \mathrm{kg}{ }^{-} 1\end{array}$ & $\begin{array}{c}\mathrm{K}, \\
\mu \mathrm{mol} \mathrm{kg}{ }^{-} 1\end{array}$ & $\begin{array}{c}\mathrm{Rb}, \\
\mathrm{nmol} \mathrm{kg}^{-1}\end{array}$ & $\begin{array}{c}\mathrm{Sr}, \\
\mathrm{nmol} \mathrm{kg}^{-1}\end{array}$ & $\begin{array}{c}\mathrm{Ba} \\
\mathrm{nmol} \mathrm{kg}^{-1}\end{array}$ \\
\hline Lake Myvatn & 11.22 & 10.60 & 0 & 0 & 1240.1 & 203.0 & 343.4 & 59.5 & 33.90 & b.d. & 15.6 \\
\hline Skaftarska & 3.64 & 1.76 & 87 & 79 & 272.0 & 78.3 & 160.0 & 8.9 & b.d. & b.d. & b.d. \\
\hline Homsa & 1.83 & 2.28 & 78 & 28 & 221.6 & 72.6 & 105.0 & 12.2 & 1.16 & b.d. & 19.5 \\
\hline Olfusa & 1.74 & 1.04 & 153 & 24 & 304.3 & 56.5 & 103.4 & 12.0 & 1.99 & b.d. & 3282.1 \\
\hline Thjorsa & 4.05 & 4.10 & 86 & 61 & 320.3 & 63.1 & 104.4 & 11.0 & b.d. & b.d. & 13.4 \\
\hline Fnjoska & 0.92 & 0.42 & 33 & 4 & 64.9 & 20.4 & 45.4 & 2.2 & b.d. & b.d. & 12.6 \\
\hline Amazon (Macapa) & 1.83 & 0.89 & 8807 & 92 & 99.6 & 67.8 & 164.5 & 29.6 & 39.95 & 152.7 & 241.1 \\
\hline Solimoes River & 2.96 & 0.65 & 13099 & 23 & 129.6 & 91.2 & 306.4 & 28.9 & 41.45 & 393.6 & 327.5 \\
\hline Negro River & 0.00 & 0.15 & 11893 & & 16.5 & 3.1 & 5.9 & 4.9 & 8.59 & b.d. & 32.6 \\
\hline Amazon (Macapa) & 1.47 & 1.93 & & & & & & & & & \\
\hline Solimoes River & 2.32 & 2.59 & & & & & & & & & \\
\hline Negro River & 0.01 & 0.20 & & & & & & & & & \\
\hline Zaire & 2.97 & 0.45 & 36 & 18 & 87.1 & 62.1 & 52.6 & 38.7 & 31.37 & 17.4 & 79.1 \\
\hline Orange & 36.58 & 24.37 & 2817 & 1209 & 3645.1 & 1094.1 & 1005.4 & 64.1 & 1.94 & 2017.4 & 333.9 \\
\hline Indus & 28.78 & 35.60 & 838 & 572 & 526.8 & 471.6 & 1063.3 & 105.4 & 7.39 & 5704.4 & 298.4 \\
\hline Meghna & 1.40 & 2.38 & 80 & 50 & 242.8 & 146.0 & 242.3 & 30.8 & 16.80 & 168.6 & 45.1 \\
\hline Brahmaputra & 5.42 & 13.21 & 44 & 192 & 217.9 & 253.4 & 700.5 & 59.1 & 28.16 & 647.5 & 99.0 \\
\hline Brahmaputra & 4.76 & 11.83 & 44 & 174 & 213.3 & 262.6 & 695.0 & 58.8 & 25.75 & 581.0 & 94.9 \\
\hline Narayani & 5.38 & 8.05 & 59 & 170 & 84.4 & 288.2 & 622.9 & 45.6 & 54.46 & 403.0 & 102.3 \\
\hline Karnali & 4.14 & 6.40 & 50 & 103 & 69.6 & 215.0 & 579.7 & 37.6 & 37.70 & 429.2 & 120.4 \\
\hline Trisuili & 1.74 & 5.38 & 33 & 69 & 70.0 & 74.2 & 368.5 & 33.2 & 44.54 & 181.0 & 23.9 \\
\hline Kosi & 2.18 & 5.74 & 31 & 62 & 68.2 & 60.7 & 303.4 & 33.7 & 37.07 & 98.0 & 20.3 \\
\hline Brahmaputra & 3.04 & 8.38 & 40 & 102 & 93.0 & 134.0 & 451.5 & 56.7 & 27.04 & 240.9 & 73.0 \\
\hline Ganga & 3.88 & 10.73 & 67 & 79 & 218.1 & 193.6 & 622.4 & 79.3 & 13.92 & 448.2 & 138.2 \\
\hline Padma & 3.34 & 9.07 & 42 & 103 & 92.8 & 126.8 & 466.2 & 58.4 & 16.42 & 291.6 & 79.1 \\
\hline Yarlung & 8.39 & 18.59 & 230 & 319 & 577.8 & 254.0 & 906.4 & 41.6 & 93.44 & 1505.7 & 58.0 \\
\hline Yangtze & 54.80 & 15.83 & 312 & 417 & 435.3 & 372.7 & 700.9 & 47.2 & 15.07 & 1713.8 & 242.7 \\
\hline Pearl & 10.94 & 12.26 & 218 & 174 & 317.9 & 65.8 & 410.7 & 83.9 & 105.91 & 172.0 & 90.3 \\
\hline Red & 13.35 & 6.69 & 68 & 126 & 166.6 & 217.5 & 744.8 & 44.4 & 28.50 & 1123.2 & 189.9 \\
\hline Fly & 52.50 & 51.21 & 12571 & 175 & 325.0 & 134.0 & 873.1 & 22.8 & 6.07 & 849.3 & 69.1 \\
\hline Fly & 52.43 & 59.21 & 17010 & 209 & 323.9 & 131.3 & 873.7 & 23.2 & 6.66 & 948.4 & 70.2 \\
\hline Purari & 3.42 & 3.89 & 13471 & 88 & 796.8 & 282.3 & 725.9 & 42.0 & 13.69 & 686.7 & 54.3 \\
\hline
\end{tabular}




\begin{tabular}{|c|c|c|c|c|c|c|c|c|c|c|c|}
\hline River & $\begin{array}{c}\text { Re, } \\
\text { pmol kg }\end{array}$ & $\begin{array}{c}\text { Mo, } \\
\mathrm{nmol} \mathrm{kg}^{-1}\end{array}$ & $\begin{array}{c}\mathrm{Cl}, \\
\mu \mathrm{mol} \mathrm{kg}{ }^{-} 1\end{array}$ & $\begin{array}{c}\mathrm{SO}_{4} \\
\mu \mathrm{mol} \mathrm{kg}{ }^{-} 1\end{array}$ & $\begin{array}{c}\mathrm{Na} \\
\mu \mathrm{mol} \mathrm{kg}-1\end{array}$ & $\begin{array}{c}\mathrm{Mg}, \\
\mu \mathrm{mol} \mathrm{kg}{ }^{-} 1\end{array}$ & $\begin{array}{c}\mathrm{Ca} \\
\mu \mathrm{mol} \mathrm{kg}{ }^{-} 1\end{array}$ & $\begin{array}{c}\mathrm{K}, \\
\mu \mathrm{mol} \mathrm{kg}-1\end{array}$ & $\begin{array}{c}\mathrm{Rb}, \\
\mathrm{nmol} \mathrm{kg}^{-1}\end{array}$ & $\begin{array}{c}\mathrm{Sr}, \\
\mathrm{nmol} \mathrm{kg}^{-1}\end{array}$ & $\begin{array}{c}\mathrm{Ba}, \\
\mathrm{nmol} \mathrm{kg}^{-1}\end{array}$ \\
\hline Purari & 3.44 & 4.01 & 13763 & 87 & 709.2 & 273.6 & 700.9 & 38.1 & 12.07 & 672.5 & 52.3 \\
\hline Kikori & 9.11 & 3.24 & 12408 & 17 & 59.7 & 283.8 & 1048.7 & 9.9 & 6.29 & 959.9 & 40.6 \\
\hline Kikori & 9.43 & 3.83 & & & 50.3 & 278.4 & 1046.3 & 8.8 & 4.54 & 830.6 & 33.9 \\
\hline Fly & 52.84 & 61.69 & & & 98.7 & 104.7 & 871.4 & 18.1 & 5.10 & 857.7 & 69.7 \\
\hline Fly & 52.33 & 62.22 & & & 90.3 & 103.6 & 864.4 & 16.0 & 4.39 & 957.3 & 71.0 \\
\hline Sepik & 1.64 & 2.02 & & & 606.9 & 242.6 & 249.1 & 23.5 & 14.29 & 181.9 & 89.5 \\
\hline Waiahole Stream & 0.49 & 0.80 & 322 & 20 & 371.6 & 176.8 & 153.8 & 14.0 & 4.58 & 88.1 & 33.7 \\
\hline Waimea Falls Pond & 0.42 & 0.30 & 422 & 24 & 354.8 & 115.0 & 64.6 & 19.9 & 1.90 & 15.8 & 19.5 \\
\hline Waiamea Reservoir & 0.41 & 0.79 & 364 & 26 & 332.8 & 70.9 & 46.2 & 15.0 & 2.21 & b.d. & 30.2 \\
\hline Yukon & 13.44 & 12.03 & 65 & 284 & 124.1 & 296.7 & 791.5 & 38.4 & 20.82 & 637.0 & 234.2 \\
\hline Copper & 9.29 & 14.43 & 53 & 215 & 104.9 & 106.8 & 518.1 & 36.1 & 10.91 & 220.3 & 85.4 \\
\hline Thompson & 4.25 & 7.12 & 19 & 88 & 79.7 & 85.5 & 322.4 & 21.3 & 16.34 & 426.7 & 46.0 \\
\hline Nechako & 16.25 & 19.63 & 17 & 47 & 101.5 & 166.3 & 375.5 & 16.6 & 3.46 & 391.4 & 79.3 \\
\hline Upper Fraser & 0.92 & 0.96 & 8 & 134 & 23.2 & 200.6 & 434.2 & 4.4 & b.d. & 820.2 & 56.6 \\
\hline Fraser & 2.90 & 3.03 & 9 & 131 & 43.4 & 201.6 & 616.4 & 12.3 & 11.53 & 793.2 & 64.1 \\
\hline Quesnel & 3.00 & 3.43 & 3 & 82 & 36.1 & 100.2 & 499.2 & 9.7 & 4.52 & 908.5 & 35.7 \\
\hline Harrison & 4.48 & 8.31 & 15 & 51 & 49.2 & 24.8 & 155.7 & 14.4 & 5.60 & 78.0 & 48.8 \\
\hline Blackwater & 6.01 & 12.03 & 12 & 21 & 275.8 & 427.7 & 461.4 & 60.9 & 18.98 & 495.3 & 43.9 \\
\hline North Thompson & 2.60 & 5.99 & 8 & 73 & 39.1 & 62.7 & 289.2 & 16.8 & 16.76 & 366.9 & 36.1 \\
\hline Nechako & 14.64 & 19.48 & 12 & 47 & 103.8 & 167.2 & 370.9 & 16.8 & 3.34 & 411.9 & 80.2 \\
\hline Fraser & 5.29 & 7.80 & 22 & 101 & 97.7 & 127.8 & 410.6 & 16.4 & 9.51 & 503.1 & 57.3 \\
\hline Fraser & 5.20 & 7.93 & 20 & 94 & 90.7 & 124.1 & 405.8 & 16.3 & 9.62 & 480.2 & 56.1 \\
\hline Andalien & 13.72 & 1.35 & 162 & 10 & 580.2 & 177.2 & 356.3 & 42.8 & 16.99 & 921.2 & 70.2 \\
\hline Bio Bio & 5.69 & 2.07 & 88 & 50 & 249.8 & 112.8 & 223.2 & 26.9 & 22.05 & 321.8 & 19.0 \\
\hline Bio Bio & 6.56 & 2.00 & 89 & 51 & 238.2 & 99.2 & 179.1 & 25.2 & 22.72 & 273.0 & 18.2 \\
\hline Andalien & 0.67 & 1.16 & 144 & 2 & 448.0 & 147.1 & 286.5 & 29.1 & 12.25 & 752.6 & 42.9 \\
\hline Tinguiririca & 17.00 & 12.58 & 161 & 366 & 328.1 & 109.6 & 544.8 & 26.1 & 38.58 & 834.6 & 25.3 \\
\hline Bio Bio & 3.48 & 2.16 & 67 & 28 & 175.7 & 92.8 & 142.3 & 22.0 & 17.43 & 230.9 & 16.3 \\
\hline Tolten & 1.55 & 3.28 & 40 & 16 & 135.7 & 55.6 & 95.7 & 18.6 & 17.25 & 138.9 & 13.7 \\
\hline Itata & 1.60 & 2.86 & 53 & 29 & 188.8 & 93.6 & 123.8 & 21.5 & 24.33 & 275.4 & 19.7 \\
\hline Maipo & 53.36 & 32.55 & 2493 & 3298 & 2985.6 & 386.7 & 3687.5 & 69.6 & 73.88 & 10055.0 & 85.6 \\
\hline Tinguiririca & 23.69 & 16.31 & 117 & 543 & 238.7 & 113.8 & 470.6 & 30.5 & 42.86 & 554.9 & 21.3 \\
\hline Maule & 7.41 & 19.36 & 193 & 174 & 364.7 & 128.7 & 284.8 & 33.1 & 45.81 & 436.0 & 22.3 \\
\hline Bio Bio & 3.76 & 2.93 & 136 & 59 & 210.2 & 93.2 & 208.1 & 28.4 & 32.39 & 261.2 & 20.1 \\
\hline
\end{tabular}




\begin{tabular}{|c|c|c|c|c|c|c|c|c|c|c|c|}
\hline River & $\begin{array}{c}\mathrm{Re}, \\
\mathrm{pmol} \mathrm{kg}^{-1}\end{array}$ & $\begin{array}{c}\text { Mo, } \\
\mathrm{nmol} \mathrm{kg}^{-1}\end{array}$ & $\begin{array}{c}\mathrm{Cl}, \\
\mu \mathrm{mol} \mathrm{kg}{ }^{-} 1\end{array}$ & $\begin{array}{c}\mathrm{SO}_{4}, \\
\mu \mathrm{mol} \mathrm{kg}{ }^{-} 1\end{array}$ & $\begin{array}{c}\mathrm{Na}, \\
\mu \mathrm{mol} \mathrm{kg}{ }^{-} 1\end{array}$ & $\begin{array}{c}\mathrm{Mg}, \\
\mu \mathrm{mol} \mathrm{kg}{ }^{-} 1\end{array}$ & $\begin{array}{c}\mathrm{Ca}, \\
\mu \mathrm{mol} \mathrm{kg}{ }^{-} 1\end{array}$ & $\begin{array}{c}\mathrm{K}, \\
\mu \mathrm{mol} \mathrm{kg}{ }^{-} 1\end{array}$ & $\begin{array}{c}\mathrm{Rb} \\
\mathrm{nmol} \mathrm{kg}\end{array}$ & $\begin{array}{c}\mathrm{Sr}, \\
\mathrm{nmol} \mathrm{kg}^{-1}\end{array}$ & $\begin{array}{c}\mathrm{Ba}, \\
\mathrm{nmol} \mathrm{kg}^{-1}\end{array}$ \\
\hline Itata & 0.69 & 6.47 & 332 & 4 & 905.2 & 471.7 & 428.4 & 46.1 & 3.03 & 1503.6 & 125.9 \\
\hline Tolten & 2.21 & 5.55 & 49 & 28 & 202.4 & 75.1 & 126.2 & 24.7 & 22.50 & 155.3 & 24.5 \\
\hline Tolten & 1.88 & 5.10 & 35 & 19 & 159.1 & 66.4 & 115.8 & 22.5 & 22.39 & 182.4 & 12.5 \\
\hline Thermal baths & 2.78 & 68.03 & 2786 & 606 & 3789.2 & 33.8 & 319.6 & 20.6 & 39.72 & 213.4 & 8.7 \\
\hline glacial river to Huinay & 3.83 & 5.04 & 28 & 24 & 47.2 & 45.0 & 246.1 & 165.0 & 19.91 & 1732.1 & 4.4 \\
\hline Pelorus & 0.59 & 1.93 & 85 & 21 & 142.0 & 105.3 & 163.1 & 8.1 & b.d. & 68.5 & 19.8 \\
\hline Danube at Regensburg & 43.05 & 18.53 & 792 & 360 & 846.7 & 660.0 & 941.3 & 77.0 & 20.05 & 1482.3 & 150.5 \\
\hline March at Angern & 52.27 & 9.61 & 1030 & 1050 & 1222.5 & 746.2 & 1581.2 & 170.0 & 30.02 & 2208.0 & 209.0 \\
\hline Danube at Passau & 35.03 & 14.43 & 794 & 334 & 68.8 & 670.3 & 1281.5 & 6.9 & 1.72 & 1633.7 & 153.8 \\
\hline Danube at Ulm & 152.25 & 32.75 & 1033 & 293 & 732.8 & 511.6 & 1027.7 & 62.2 & 7.76 & 1488.1 & 162.7 \\
\hline Regen at Regensburg & 12.68 & 2.33 & 601 & 132 & 489.6 & 188.5 & 521.7 & 56.0 & 23.12 & 305.7 & 87.6 \\
\hline Tisza at Szeged & 22.47 & 7.81 & 1193 & 402 & 1491.6 & 359.5 & 1104.7 & 73.7 & 16.82 & 1154.1 & 129.5 \\
\hline Danube at Vienna & 21.08 & 11.84 & 503 & 325 & 538.6 & 498.0 & 1128.5 & 57.1 & 22.63 & 1527.1 & 108.3 \\
\hline Inn at Schärding & 13.89 & 12.97 & 277 & 238 & 301.3 & 381.4 & 914.9 & 40.3 & 21.25 & 1388.7 & 85.2 \\
\hline Iller at Wiblinger & 24.32 & 7.21 & 721 & 192 & 474.1 & 603.4 & 829.3 & 49.5 & 3.52 & 2626.1 & 205.3 \\
\hline Duna at Budapest & 74.30 & 10.76 & 1832 & 1032 & 2024.3 & 1196.4 & 1853.1 & 161.6 & 31.84 & 3255.6 & 403.1 \\
\hline
\end{tabular}

Table 2.3: B: Sample ID and chemical data for tributary and exorheic river samples. 


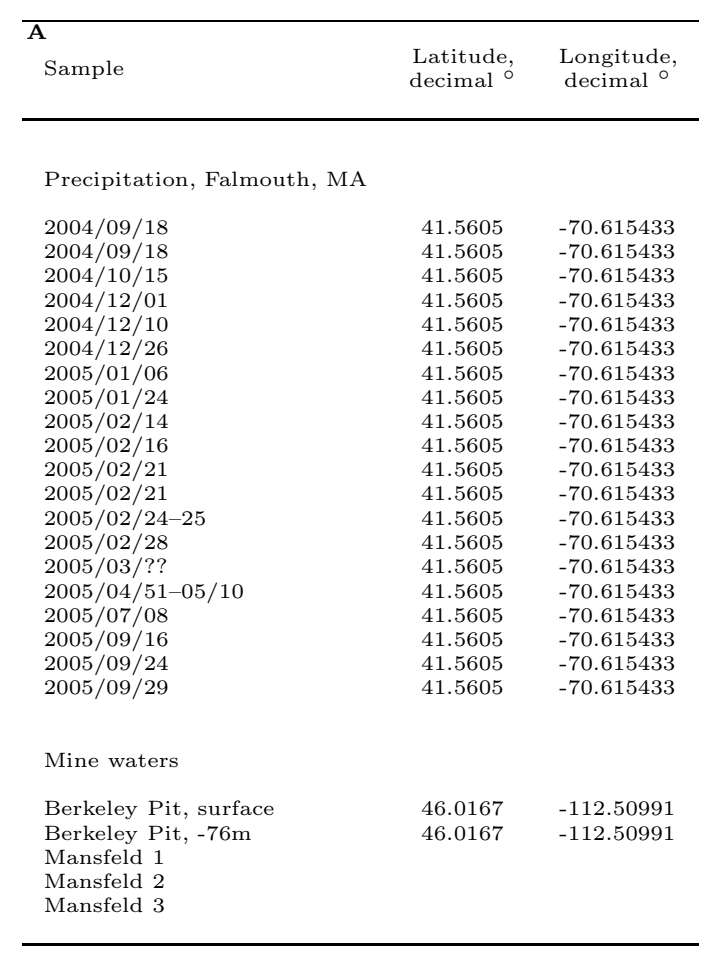

Table 2.4: A: Locations of precipitation and mine water samples. All precipitation samples are from Falmouth, MA. Berkeley Pit samples are from Butte, MT; Mansfeld samples are from a mine of the Kupferschiefer near Mansfeld, Germany. 


\begin{tabular}{|c|c|c|c|c|c|c|c|c|c|c|c|}
\hline Sample & $\begin{array}{c}\text { Re, } \\
\text { pmol kg }^{-1}\end{array}$ & $\begin{array}{c}\mathrm{Mo}, \\
\mathrm{nmol} \mathrm{kg}^{-1}\end{array}$ & $\begin{array}{c}\mathrm{Cl}, \\
\mu \mathrm{mol} \mathrm{kg}{ }^{-} 1\end{array}$ & $\begin{array}{c}\mathrm{SO} 4, \\
\mu \mathrm{mol} \mathrm{kg}{ }^{-} 1\end{array}$ & $\begin{array}{c}\mathrm{Na}, \\
\mu \mathrm{mol} \mathrm{kg}^{-}{ }^{1}\end{array}$ & $\begin{array}{c}\mathrm{Mg}, \\
\mu \mathrm{mol} \mathrm{kg}^{-} 1\end{array}$ & $\begin{array}{c}\mathrm{Ca}, \\
\mu \mathrm{mol} \mathrm{kg}\end{array}$ & $\begin{array}{c}\mathrm{K}, \\
\mu \mathrm{mol} \mathrm{kg}{ }^{-} 1\end{array}$ & $\begin{array}{c}\mathrm{Rb}, \\
\mathrm{nmol} \mathrm{kg}\end{array}$ & $\begin{array}{c}\mathrm{Sr}, \\
\mathrm{nmol} \mathrm{kg}^{-1}\end{array}$ & $\begin{array}{c}\mathrm{Ba}, \\
\mathrm{nmol} \mathrm{kg}^{-1}\end{array}$ \\
\hline \multicolumn{12}{|c|}{ Precipitation, Falmouth, MA } \\
\hline $2004 / 09 / 18$ & 1.41 & 0.08 & 103 & 33 & 80 & 10.6 & 7.1 & 4.6 & b.d & b.d & 43 \\
\hline $2004 / 09 / 18$ & $\begin{array}{l}1.41 \\
0.91\end{array}$ & 0.08 & 23 & 4.9 & 4.3 & 2.20 & 4.6 & 1.4 & b.d & b.d & b.d \\
\hline $2004 / 10 / 15$ & 0.81 & 0.05 & 26 & 8.0 & b.d & 2.97 & 7.5 & 3.9 & b.d & b.d & 20 \\
\hline $2004 / 12 / 01$ & 1.25 & 0.64 & 204 & 17.0 & 140 & 19.4 & 7.6 & 5.6 & b.d & b.d & 16 \\
\hline $2004 / 12 / 10$ & 0.70 & 0.06 & 26 & 7.2 & b.d & 2.79 & 5.8 & b.d & b.d & b.d & 33 \\
\hline $2004 / 12 / 26$ & 0.29 & 0.02 & 16.4 & 2.4 & b.d & 1.64 & 7.1 & b.d & b.d & b.d & 44 \\
\hline $2005 / 01 / 06$ & 1.03 & 0.07 & 9.8 & 4.5 & b.d & 0.98 & 4.1 & b.d & b.d & b.d & 71 \\
\hline $2005 / 01 / 24$ & 0.17 & 0.06 & 57 & 4.7 & 23 & 5.63 & 5.8 & b.d & b.d & b.d & 19 \\
\hline $2005 / 02 / 14$ & 1.15 & 0.15 & 9.8 & 19 & b.d & 1.19 & 5.3 & b.d & b.d & b.d & 25 \\
\hline $2005 / 02 / 16$ & 5.9 & 0.14 & 440 & 32 & 320 & 39.9 & 25 & 19 & 12 & b.d & 21 \\
\hline $2005 / 02 / 21$ & 1.13 & 0.14 & 31 & 7.9 & 7 & 3.31 & 3.7 & b.d & b.d & b.d & 35 \\
\hline $2005 / 02 / 21$ & 0.57 & b.d & 14 & 3.7 & b.d & 1.82 & 3.9 & b.d & b.d & b.d & b.d \\
\hline $2005 / 02 / 24-25$ & 5.9 & 0.14 & 14 & 2.0 & b.d & 2.54 & 6.6 & b.d & b.d & b.d & b.d \\
\hline $2005 / 02 / 28$ & 1.13 & 0.14 & 20 & 3.1 & b.d & 2.53 & 4.7 & b.d & b.d & b.d & 28 \\
\hline $2005 / 03 / ? ?$ & 0.30 & 0.06 & 71 & 7.0 & 36 & 6.87 & 8.1 & 1.6 & b.d & b.d & 48 \\
\hline $2005 / 04 / 51-2005 / 05 / 10$ & 2.4 & 0.02 & 95 & 16.6 & 65 & 9.1 & 8.8 & $\begin{array}{l}3.1 \\
3.1\end{array}$ & b.d & b.d & 22 \\
\hline $2005 / 07 / 08$ & 0.35 & 1.28 & 19 & 7.9 & b.d & 1.73 & 8.0 & 0.7 & b.d & b.d & 20 . \\
\hline $2005 / 09 / 16$ & 0.03 & b.d & 1.8 & 1.0 & b.d & 0.42 & 4.3 & b.d & b.d & b.d & $1.00 \times 10^{3}$ \\
\hline $2005 / 09 / 24$ & 0.27 & b.d & 71 & 8.5 & 46 & 7.16 & 6.6 & 3.5 & b.d & b.d & 69 \\
\hline $2005 / 09 / 29$ & 2.2 & 0.06 & 360 & 42 & 280 & 35.4 & 21 & b.d & 10. & b.d & 17 \\
\hline \multicolumn{12}{|l|}{ Mine waters } \\
\hline Berkeley Pit, surface & 11900 & 0.76 & 710 & $8.0 \times 10^{5}$ & 6.7 & 268 & 11000 & 90. & 590 & b.d & 930 \\
\hline Berkeley Pit, $-76 \mathrm{~m}$ & $1.31 \times 10^{4}$ & 0.62 & 550 & 86000 & 5.1 & 132 & 15000 & 2.6 & 270 & b.d & 410 \\
\hline Mansfeld 1 & 14400 & 220 & & & 43 & 4330 & 16000 & $7.0 \times 10^{2}$ & 470 & 19000 & 120 \\
\hline Mansfeld 2 & 37100 & 250 & & & 2400 & 4650 & 14000 & $7.0 \times 10^{2}$ & 570 & 33000 & 150 \\
\hline Mansfeld 3 & 16200 & 190 & & & $2.0 \times 10^{4}$ & 7070 & $2.0 \times 10^{4}$ & 1200 & $8.0 \times 10^{2}$ & 14000 & 170 \\
\hline
\end{tabular}

Table 2.4: B: Chemical data for precipitation and mine water samples. Chemical data are listed to the last significant digit. Where the last significant digit is zero, this is indicated by a decimal point or scientific notation. Uncertainties are as listed in Section 2.3 . 


\begin{tabular}{|c|c|c|c|c|c|c|c|}
\hline $\begin{array}{l}\text { Location, date } \\
\qquad \mathrm{yr} / \mathrm{m} / \mathrm{d}\end{array}$ & $\begin{array}{c}\mathrm{Re}, \\
\mathrm{pmol} \mathrm{kg}^{-1} \text { ) }\end{array}$ & $\begin{array}{c}\text { Mo, } \\
\mathrm{nmol} \mathrm{kg}^{-1}\end{array}$ & $\begin{array}{c}\text { Salinity, }{ }^{*} \\
\% o\end{array}$ & $\begin{array}{c}\mathrm{Cl}-, \\
\mu \mathrm{mol} \mathrm{kg}{ }^{-1}\end{array}$ & $\begin{array}{c}\mathrm{SO}_{4}^{2-} \\
\mu \mathrm{mol} \mathrm{kg}\end{array}$ & $\begin{array}{l}\text { Latitude, } \\
\text { decimal }\end{array}$ & $\begin{array}{l}\text { Longitude } \\
\text { decimal }\end{array}$ \\
\hline
\end{tabular}

Mississippi River Estuary, 2001/11/09-11

WP3 1

WP1 6

WP2 3

WP5 5

MR 5S

MR $20 \mathrm{~S}$

MR 7S

MR 2B

MR 9S

MR 2S

WP4 4.5

\section{Hudson River estuary (6 profiles)}

Profile $1,2004 / 07 / 07 \dagger$

Station 1

Station 1, 2nd analysis

Station 2

Station 2, 2nd analysis

Station 4

Station 9

Station 9, 2nd analysis

Station 11

Station 13

Station 15

Station 15, 2nd analysis

Station 20

tation 22

Station 24

Station 27

Station 29

Station 31

$\begin{array}{ccc}91 & 29 & 0.40 \\ 47 & 98 & 31.50 \\ 89 & 32 & 1.50 \\ 41 & 114 & 35.70 \\ 70 & 60 & 14.20 \\ 87 & 33 & 3.00 \\ 81 & 43 & 6.80 \\ 42 & 117 & 35.70 \\ 81 & 41 & 4.40 \\ 61 & 77 & 22.20 \\ 51 & 100 & 29.40\end{array}$

$\begin{array}{ll}29.200 & -89.275 \\ 29.127 & -89.261 \\ 29.162 & -89.254 \\ 28.882 & -89.437 \\ 28.901 & -89.429 \\ 28.970 & -89.382 \\ 28.923 & -89.415 \\ 28.847 & -89.435 \\ 29.953 & -89.393 \\ 28.847 & -89.435 \\ 28.880 & -89.428\end{array}$

$\begin{array}{lcc}38 & 83 & 17.80 \\ 38 & 84 & 17.80 \\ \mathbf{4 0} & 77 & 16.90 \\ \mathbf{4 0} & 77 & 16.90 \\ 95 & 350 & 17.30 \\ 42 & 62 & 15.80 \\ 42 & 62 & 15.80 \\ 43 & 57 & 14.75 \\ 43 & 55 & 13.00 \\ 46 & 45 & 11.20 \\ 46 & 45 & 11.20 \\ 48 & 45 & 11.70 \\ 48 & 45 & 10.60 \\ 49 & 38 & 11.80 \\ 47 & 34 & 7.60 \\ 49 & 33 & 7.70 \\ 47 & 31 & 7.20\end{array}$

$\begin{array}{ll}40.6864 & -74.0372 \\ 40.6864 & -74.0372 \\ 40.7077 & -74.0240 \\ 40.7077 & -74.0240 \\ 40.7390 & -74.0146 \\ 40.7730 & -74.0037 \\ 40.7730 & -74.0037 \\ 40.8034 & -73.9761 \\ 40.8347 & -73.9565 \\ 40.8741 & -73.9336 \\ 40.8741 & -73.9336 \\ 40.8877 & -73.9210 \\ 40.9008 & -73.9105 \\ 40.9648 & -73.8986 \\ 41.0196 & -73.8842 \\ 41.0532 & -73.8785 \\ 41.0744 & -73.8883\end{array}$




\begin{tabular}{|c|c|c|c|c|c|c|c|}
\hline $\begin{array}{l}\text { Location, date } \\
\qquad \mathrm{yr} / \mathrm{m} / \mathrm{d}\end{array}$ & $\begin{array}{c}\mathrm{Re}, \\
\mathrm{pmol} \mathrm{kg}^{-1} \text { ) }\end{array}$ & $\begin{array}{c}\text { Mo, } \\
\mathrm{nmol} \mathrm{kg}^{-1}\end{array}$ & $\begin{array}{c}\text { Salinity, }{ }^{*} \\
\% 0\end{array}$ & $\begin{array}{c}\mathrm{Cl}-, \\
\mu \mathrm{mol} \mathrm{kg}{ }^{-1}\end{array}$ & $\begin{array}{c}\mathrm{SO}_{4}^{2-} \\
\mu \mathrm{mol} \mathrm{kg}\end{array}$ & $\begin{array}{l}\text { Latitude, } \\
\text { decimal }\end{array}$ & $\begin{array}{l}\text { Longitude } \\
\text { decimal }\end{array}$ \\
\hline Station 31, 2nd analysis & 114 & 154 & 7.20 & & & 41.0744 & -73.8883 \\
\hline Station 36 & 47 & 25 & 5.70 & & & 41.1063 & -73.8813 \\
\hline Station 36,2 nd analysis & 47 & 26 & 5.70 & & & 41.1063 & -73.8813 \\
\hline Station 39 & 49 & 25 & 5.40 & & & 41.1554 & -73.8995 \\
\hline Station 44 & 45 & 19 & 3.60 & & & 41.2330 & -73.9579 \\
\hline Station 46 & 45 & 14.9 & 2.83 & & & 41.2623 & -73.9713 \\
\hline Station 48 & 43 & 11.2 & 1.78 & & & 41.2920 & -73.9531 \\
\hline Station 50 & 43 & 10.0 & 1.38 & & & 41.3163 & -73.9838 \\
\hline Station 54 & 41 & 3.6 & 0.80 & & & 41.3923 & -73.9479 \\
\hline Station 56 & 39 & 5.9 & 0.50 & & & 41.4231 & -73.9719 \\
\hline Station 56,2 nd analysis & 39 & 6.1 & 0.50 & & & 41.4231 & -73.9719 \\
\hline Station 57 & 39 & 5.9 & 0.43 & & & 41.4388 & -73.9838 \\
\hline Station 57,2 nd analysis & 39 & 5.9 & 0.43 & & & 41.4388 & -73.9838 \\
\hline Station 64 & 47 & 18.6 & 4.20 & & & 41.2330 & -73.9591 \\
\hline Station 69 & 47 & 40 & 9.40 & & & 41.0763 & -73.8822 \\
\hline Station 76 & 38 & 72 & 19.50 & & & 40.8336 & -73.9573 \\
\hline Station 76,2 nd analysis & 39 & 73 & 19.50 & & & 40.8336 & -73.9573 \\
\hline Station 76 & 40 & 75 & 19.50 & & & 40.8336 & -73.9573 \\
\hline Station 77 & 38 & 76 & 22.40 & & & 40.8029 & -73.9779 \\
\hline Station 77,2 nd analysis & 37 & 76 & 22.40 & & & 40.8029 & -73.9779 \\
\hline Station 78 & 37 & 78 & 21.40 & & & 40.7729 & -74.0033 \\
\hline Station 80 & 35 & 86 & 23.70 & & & 40.7085 & -74.0250 \\
\hline Station 81 & 34 & 93 & 25.20 & & & 40.6861 & -74.0386 \\
\hline Station 81,2 nd analysis & 34 & 92 & 25.20 & & & 40.6861 & -74.0386 \\
\hline \multicolumn{8}{|l|}{ Profile $2,2006 / 06 / 06^{\dagger}$} \\
\hline Station 1 & 8.7 & 8.8 & 1.69 & 27000 & 1,450 & 41.0437 & -73.8807 \\
\hline Station 2 & 8.2 & 7.1 & 1.15 & 18400 & 1,000 & 41.0840 & -73.8840 \\
\hline Station 3 & 7.9 & 6.1 & 0.80 & 12800 & 710 & 41.1049 & -73.8804 \\
\hline Station 4 & 7.7 & 5.3 & 0.59 & 9400 & 550 & 41.1256 & -73.8784 \\
\hline Station 5 & 7.1 & 4.7 & 0.33 & 5300 & 350 & 41.1484 & -73.8970 \\
\hline Station 6 & 7.0 & 3.8 & 0.12 & 1900 & 183 & 41.1590 & -73.9165 \\
\hline Station 7 & 7.0 & 4.0 & 0.06 & 1000 & 140 & 41.1852 & -73.9365 \\
\hline Station 8 & 7.0 & 3.1 & 0.05 & 770 & 130 & 41.2055 & -73.9470 \\
\hline Station 9 & 7.0 & 3.6 & 0.05 & 720 & 124 & 41.2216 & -73.9534 \\
\hline
\end{tabular}




\begin{tabular}{|c|c|c|c|c|c|c|c|}
\hline $\begin{array}{l}\text { Location, date } \\
\qquad \mathrm{yr} / \mathrm{m} / \mathrm{d}\end{array}$ & $\begin{array}{c}\mathrm{Re}, \\
\mathrm{pmol} \mathrm{kg}^{-1} \text { ) }\end{array}$ & $\begin{array}{c}\text { Mo, } \\
\mathrm{nmol} \mathrm{kg}^{-1}\end{array}$ & $\begin{array}{c}\text { Salinity, }{ }^{*} \\
\% 0\end{array}$ & $\begin{array}{c}\mathrm{Cl}-, \\
\mu \mathrm{mol} \mathrm{kg}{ }^{-1}\end{array}$ & $\begin{array}{c}\mathrm{SO}_{4}^{2-} \\
\mu \mathrm{mol} \mathrm{kg}\end{array}$ & $\begin{array}{l}\text { Latitude, } \\
\text { decimal }\end{array}$ & $\begin{array}{l}\text { Longitude } \\
\text { decimal }\end{array}$ \\
\hline Station 10 & 6.9 & 3.3 & 0.04 & 710 & 126 & 41.2430 & -73.9653 \\
\hline Station 11 & 7.2 & 3.2 & 0.04 & 660 & 124 & 41.2642 & -73.9700 \\
\hline Station 12 & 7.0 & & 0.04 & 640 & 120 & 41.3100 & -73.9764 \\
\hline Station 13 & 7.2 & & 0.04 & 570 & 116 & 41.3458 & -73.9612 \\
\hline Station 14 & 6.9 & & 0.04 & 560 & 115 & 41.3852 & -73.9528 \\
\hline Station 15 & 7.0 & & 0.03 & 550 & 114 & 41.3962 & -73.9483 \\
\hline Station 16 & 6.9 & & 0.03 & 540 & 113 & 41.4360 & -73.9819 \\
\hline Station 17 & 7.0 & & 0.03 & 500 & 108 & 41.5153 & -74.0015 \\
\hline Station 18 & 7.3 & & 0.03 & 510 & 111 & 41.5750 & -73.9586 \\
\hline Station 19 & 7.2 & & 0.03 & 530 & 113 & 41.6328 & -73.9480 \\
\hline Station 20 & 7.1 & & 0.03 & 520 & 111 & 41.6513 & -73.9333 \\
\hline Station 21 & 7.2 & & 0.03 & 490 & 108 & 41.7239 & -73.9430 \\
\hline \multicolumn{8}{|l|}{ Profile $3,2006 / 10 / 12^{\dagger}$} \\
\hline NY Harbor, Breezy Point & 24 & 80 & 28.85 & 460,000 & 21,000 & 40.5575 & -73.9328 \\
\hline NY Harbor, Fort Wadsworth & 29 & 95 & 25.09 & 400,000 & 18,300 & 40.5992 & -74.0544 \\
\hline NY Harbor, Red Hook, Brooklyn & 27 & 80 & 20.70 & 330,000 & 15,000 & 40.6784 & -74.0190 \\
\hline RM ER 1E, East River, Empire Fulton Ferry & 26 & 84 & 21.95 & 350,000 & 18,100 & 40.7049 & -73.9903 \\
\hline RM ER 1W, East River, Lower East Side Ecology Center & 26 & 87 & 22.58 & 360,000 & 19,000 & 40.7042 & -73.9948 \\
\hline RM ER 4, Solar One & 27 & 86 & 22.58 & 360,000 & 17,800 & 40.7365 & -73.9743 \\
\hline RM 2, Pier 40 & 26 & 72 & 18.19 & 290,000 & 14,500 & 40.7211 & -74.0131 \\
\hline RM 7, 79th Street Boat Basin & 24 & 50 & 11.92 & 190,000 & 9,500 & 40.7863 & -73.9860 \\
\hline RM 13, Harlem River, Swindler Cove & 24 & 56 & 13.17 & 210,000 & 9,100 & 40.8569 & -73.9219 \\
\hline RM 14, Harlem River, Inwood Hill Park & 25 & 53 & 12.54 & 200,000 & 10,000 & 40.8736 & -73.9209 \\
\hline RM 11.5, Fort Washington Park & 23 & 38 & 9.28 & 148,000 & 5,400 & 40.8450 & -73.9461 \\
\hline RM 18, Beczak & 22 & 35 & 7.46 & 119,000 & 6,200 & 40.9379 & -73.9032 \\
\hline RM 18.5, Alpine Boat Basin & 24 & 32 & 6.71 & 107,000 & 5,600 & 40.9481 & -73.9182 \\
\hline RM 25E, Matheson Park, Irvington & 24 & 27 & 5.52 & 88,000 & 4,400 & 41.0426 & -73.8740 \\
\hline RM 25W, Piermont Pier & 20 & 23 & 4.20 & 67,000 & 3,500 & 41.0431 & -73.8989 \\
\hline RM 35, Croton Point Park & 23 & 17.5 & 2.82 & 45,000 & 2,400 & 41.1787 & -73.8943 \\
\hline RM 39.5, George's Island, Montrose & 23 & 15.1 & 2.13 & 34,000 & 1,700 & 41.2348 & -73.9434 \\
\hline RM 41, Verplanck & 24 & 12.3 & 1.38 & 22,000 & 1,500 & 41.2481 & -73.9631 \\
\hline RM 55, Little Stony Point, Cold Spring & 26 & 7.3 & 0.10 & 1,540 & 210 & 41.4278 & -73.9691 \\
\hline RM 58, Kowawese, New Windsor & 29 & 6.6 & 0.06 & 940 & 184 & 41.4624 & -74.0122 \\
\hline RM 61E, Long Dock, Beacon & 26 & 7.3 & 0.07 & 1,050 & 190 & 41.5038 & -73.9885 \\
\hline
\end{tabular}




\begin{tabular}{|c|c|c|c|c|c|c|c|}
\hline $\begin{array}{l}\text { Location, date } \\
\qquad \mathrm{yr} / \mathrm{m} / \mathrm{d}\end{array}$ & $\begin{array}{c}\mathrm{Re}, \\
\mathrm{pmol} \mathrm{kg}^{-1} \text { ) }\end{array}$ & $\begin{array}{c}\text { Mo, } \\
\mathrm{nmol} \mathrm{kg}^{-1}\end{array}$ & $\begin{array}{c}\text { Salinity, }{ }^{*} \\
\% 0\end{array}$ & $\begin{array}{c}\mathrm{Cl}-, \\
\mu \mathrm{mol} \mathrm{kg}^{-1}\end{array}$ & $\begin{array}{c}\mathrm{SO}_{4}^{2-} \\
\mu \mathrm{mol} \mathrm{kg}\end{array}$ & $\begin{array}{l}\text { Latitude, } \\
\text { decimal }\end{array}$ & $\begin{array}{l}\text { Longitude } \\
\text { decimal }\end{array}$ \\
\hline RM $61 \mathrm{~W}$, Newburgh Landing & 33 & 7.1 & 0.05 & 800 & 173 & & \\
\hline RM 76, Waryas Park, Poughkeepsie & 134 & 23 & 0.06 & 980 & 175 & 41.7066 & -73.9404 \\
\hline RM 76, Clearwater (boat), Poughkeepsie & 45 & 6.3 & 0.05 & 810 & 174 & 41.7067 & -73.9448 \\
\hline RM 84.5, Norrie Point, Staatsburgh & 46 & 7.3 & 0.05 & 790 & 174 & 41.8363 & -73.9408 \\
\hline RM 87, Esopus Meadows, Ulster Park & 43 & 6.4 & 0.05 & 750 & 169 & 41.8681 & -73.9508 \\
\hline RM 92, Kingston Point & 48 & 7.0 & 0.05 & 810 & 190 & 41.9273 & -73.9631 \\
\hline RM 102, Saugerties Lighthouse & 59 & 6.2 & 0.05 & 780 & 184 & 42.0898 & -73.9370 \\
\hline RM 115, Cohotate Preserve, Athens & 26 & 5.6 & 0.04 & 680 & 170 & 42.2511 & -73.8157 \\
\hline RM 124.5, Nutten Hook, Stuyvesant & 16 & 5.6 & 0.04 & 640 & 164 & 42.3536 & -73.7894 \\
\hline RM 127, Stuyvesant Landing & 16 & 5.7 & 0.04 & 680 & 165 & 42.3869 & -73.7859 \\
\hline RM 153, Green Island Park, Troy & 10 & 5.1 & 0.04 & 580 & 151 & 42.7464 & -73.6891 \\
\hline \multicolumn{8}{|l|}{ Profile $4,2006 / 11 / 05^{\dagger}$} \\
\hline Station 1 & 28 & 66 & 23.5 & & & 40.7024 & -74.0262 \\
\hline Station 2 & 26 & 43 & 16.0 & & & 40.7352 & -74.0193 \\
\hline Station 3 & 25 & 43 & 15.0 & & & 40.7671 & -74.0070 \\
\hline Station 4 & 26 & 43 & 13.5 & & & 40.7983 & -73.9834 \\
\hline Station 5 & 26 & 40 & 13.7 & & & 40.8276 & -73.9612 \\
\hline Station 6 & 25 & 39 & 14.0 & & & 40.8595 & -73.9437 \\
\hline Station 7 & 25 & 35 & 10.8 & & & 40.8888 & -73.9240 \\
\hline Station 8 & 25 & 33 & 10.0 & & & 40.9220 & -73.9136 \\
\hline Station 9 & 24 & 30 & 8.3 & & & 40.9559 & -73.9031 \\
\hline Station 10 & 25 & 26 & 8.3 & & & 40.9872 & -73.8931 \\
\hline Station 11 & 24 & 21 & 6.8 & & & 41.0216 & -73.8838 \\
\hline Station 12 & 24 & 21 & 6.2 & & & 41.0546 & -73.8798 \\
\hline Station 13 & 25 & 19 & 5.7 & & & 41.0885 & -73.8828 \\
\hline Station 14 & 26 & 14.7 & 3.7 & & & 41.1392 & -73.8845 \\
\hline Station 15 & 27 & 12.8 & 2.8 & & & 41.1614 & -73.9086 \\
\hline \multicolumn{8}{|l|}{ Profile $5,2007 / 10 / 02{ }^{\dagger}$} \\
\hline NY Harbor, Breezy Point & 30 & 49 & & & & 40.5575 & -73.9328 \\
\hline NY Harbor, Fort Wadsworth & 29 & 43 & & & & 40.6053 & -74.0537 \\
\hline RM ER 0.5, Southstreet Seaport Museum & 30 & 37 & & & & 40.7550 & -74.0033 \\
\hline RM ER 1E, East River, Empire Fulton Ferry & 30 & 43 & & & & 40.7049 & -73.9903 \\
\hline
\end{tabular}




\begin{tabular}{|c|c|c|c|c|c|c|c|}
\hline $\begin{array}{l}\text { Location, date } \\
\qquad \mathrm{yr} / \mathrm{m} / \mathrm{d}\end{array}$ & $\begin{array}{c}\mathrm{Re}, \\
\mathrm{pmol} \mathrm{kg}^{-1} \text { ) }\end{array}$ & $\begin{array}{c}\text { Mo, } \\
\mathrm{nmol} \mathrm{kg}^{-1}\end{array}$ & $\begin{array}{c}\text { Salinity, }{ }^{*} \\
\% 0\end{array}$ & $\begin{array}{c}\mathrm{Cl}-, \\
\mu \mathrm{mol} \mathrm{kg}-1\end{array}$ & $\begin{array}{c}\mathrm{SO}_{4}^{2-} \\
\mu \mathrm{mol} \mathrm{kg}\end{array}$ & $\begin{array}{l}\text { Latitude, } \\
\text { decimal }\end{array}$ & $\begin{array}{l}\text { Longitude } \\
\text { decimal }\end{array}$ \\
\hline RM ER 4, Solar One & 29 & 45 & & & & 40.7333 & -73.9667 \\
\hline RM ER 4, Gantry Plaza State Park & 30 & 43 & & & & 40.7333 & -73.9667 \\
\hline RM 2, Pier 40 & 36 & 31 & & & & 40.7211 & -74.0131 \\
\hline RM 2.5, Christopher Street, Pier 45 & 34 & 39 & & & & 40.7335 & -74.0127 \\
\hline RM 7, 79th Street Boat Basin & 37 & 37 & & & & 40.7863 & -73.9860 \\
\hline RM 12, Lettie G. Howard, b/w 79th St and G. W. Bridge & 37 & 43 & & & & 40.8183 & -73.9699 \\
\hline RM 13a, Harlem River, Swindler Cove & 42 & 23 & & & & 40.8570 & -73.9219 \\
\hline RM 14, Harlem River, Inwood Hill Park & 45 & 38 & & & & 40.8794 & -73.9211 \\
\hline RM 18, Beczak & 47 & 28 & & & & 40.9379 & -73.9032 \\
\hline RM 18.5, Alpine Boat Basin & 45 & 20 & & & & 40.9500 & -73.9167 \\
\hline RM 25W, Piermont Pier & 48 & 18 & & & & 41.0429 & -73.8961 \\
\hline RM 28 Nyack Brook at Nyack & 15 & 4.6 & & & & 41.0874 & -73.9165 \\
\hline RM 28, Nyack Memorial Beach & 71 & 30 & & & & 41.0874 & -73.9165 \\
\hline RM 32, Ossining (bottle 1) & 54 & 22 & & & & 41.1548 & -73.8693 \\
\hline RM 32, Ossining (bottle 2) & 53 & 18 & & & & 41.1548 & -73.8693 \\
\hline RM 35, Croton Point Park & 56 & 19 & & & & 41.1787 & -73.8943 \\
\hline RM 39.5, George's Island, Montrose & 58 & 20 & & & & 41.2333 & -73.9444 \\
\hline RM 41, Verplanck & 64 & 17.0 & & & & 41.2493 & -73.9646 \\
\hline RM 55, Little Stony Point, Cold Spring & 81 & 10.3 & & & & 41.4278 & -73.9691 \\
\hline RM 58, Kowawese, New Windsor & 83 & 10.1 & & & & 41.4624 & -74.0122 \\
\hline RM 61E, Long Dock, Beacon & 87 & 9.1 & & & & 41.5038 & -73.9885 \\
\hline RM 76, Waryas Park, Poughkeepsie & 114 & 5.1 & & & & 41.7066 & -73.9404 \\
\hline RM 78, Quiet Cove, Poughkeepsie & 120 & 5.0 & & & & 41.7333 & -73.9333 \\
\hline RM 84.5, Norrie Point, Staatsburgh & 125 & 3.5 & & & & 41.8316 & -73.9421 \\
\hline RM 92, Kingston Point & 170 & 3.9 & & & & 41.9267 & -73.9633 \\
\hline RM 96.5, Ulster Landing State Park & 181 & 4.9 & & & & 42.0047 & -73.9440 \\
\hline RM 102, Saugerties Lighthouse & 173 & 4.6 & & & & 42.0898 & -73.9370 \\
\hline RM 112, Dutchman's Landing Park, Catskill & 174 & 3.5 & & & & 42.2100 & -73.9200 \\
\hline RM 115, Cohotate Preserve, Athens & 184 & 4.1 & & & & 42.2511 & -73.8157 \\
\hline RM 123, Coxsackie State Park & 181 & 3.4 & & & & 42.3428 & -73.7892 \\
\hline RM 127, Stuyvesant Landing & 190 & 4.4 & & & & 42.3869 & -73.7859 \\
\hline RM 133, Schodack Island State Park & 230 & 4.1 & & & & 42.5003 & -73.7766 \\
\hline RM 146, Half-Moon Site, Albany & 290 & 3.4 & & & & 42.6494 & -73.7467 \\
\hline RM 153, Green Island Park, Troy & 440 & 1.24 & & & & 42.7464 & -73.6891 \\
\hline
\end{tabular}




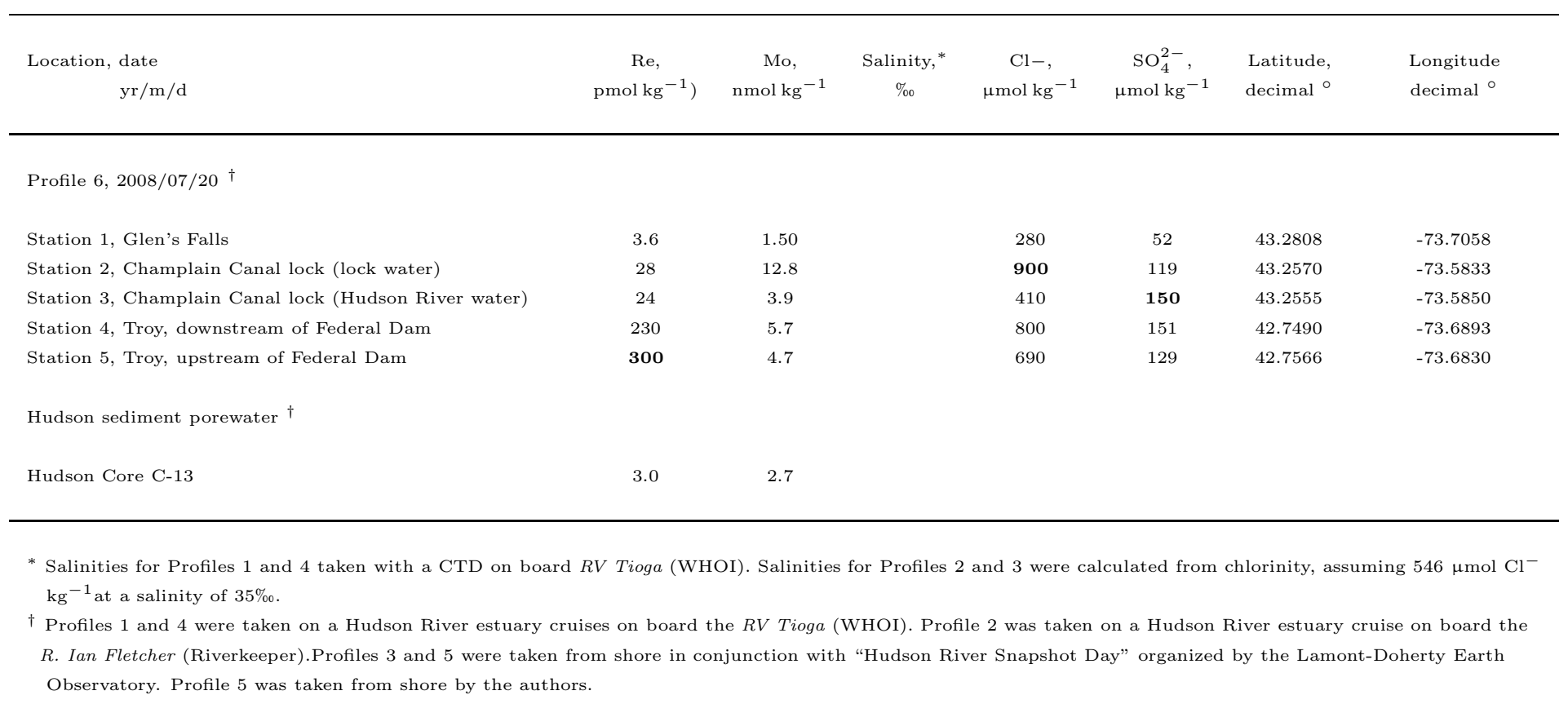

Table 2.5: Listing, locations, dates and chemical data for one Mississippi estuary profile and six Hudson estuary profiles 


\begin{tabular}{|c|c|c|c|c|c|c|c|c|c|c|c|c|}
\hline Sample & $\begin{array}{c}\text { Mo, } \\
\mathrm{nmol} \mathrm{kg}^{-1}\end{array}$ & $\begin{array}{c}\mathrm{Re}, \\
\text { pmol kg }^{-1}\end{array}$ & $\begin{array}{l}\text { Sol'n mass, } \\
\mathrm{g}\end{array}$ & $\begin{array}{c}\text { Total Mo, }{ }^{*} \\
\text { nmol }\end{array}$ & $\begin{array}{c}\text { Total Re, }{ }^{*} \\
\text { pmol }\end{array}$ & $\begin{array}{c}\text { Total Mo, } \\
\text { nmol }\end{array}$ & $\begin{array}{c}\text { Total Re, } \\
\text { pmol }\end{array}$ & $\begin{array}{c}\mathrm{Mo}^{\dagger} \\
\mathrm{nmol} \mathrm{kg}\end{array}$ & $\begin{array}{c}\mathrm{Re}^{\dagger}{ }^{\dagger} \\
\mathrm{pmol} \mathrm{kg}^{-1}\end{array}$ & $\begin{array}{c}\mathrm{Mg}_{,}^{\ddagger} \\
\mathrm{mmol} \mathrm{kg}\end{array}$ & $\begin{array}{c}\text { Mg-corr Mo Mo } \$ \\
\text { nmol kg-1 }\end{array}$ & $\begin{array}{c}\text { Mg-corr Re, }{ }^{,} \\
\text {pmol kg }^{-1}\end{array}$ \\
\hline $\begin{array}{l}\text { J2 } 208 \text { M2 Fluid } \\
\text { J2 } 208 \text { M2 Dregs } \\
\text { J2 } 208 \text { M2 Bttl Fltrt } \\
\text { 乏 J2 } 208 \text { M2 }\end{array}$ & $\begin{array}{c}3.3 \\
630 \\
82.2\end{array}$ & $\begin{array}{r}14.4 \\
8.9 \\
97.4\end{array}$ & $\begin{array}{l}750 \\
25.351 \\
29.1032\end{array}$ & $\begin{array}{c}2.5 \\
16 \\
4.61\end{array}$ & $\begin{array}{c}10.8 \\
0.22 \\
5.46\end{array}$ & 23 & 16 & 31 & 22 & 27 & -27 & 5.6 \\
\hline $\begin{array}{l}\text { J2 } 208 \text { M4 Fluid } \\
\text { J2 } 208 \text { M4 Dregs } \\
\text { J2 208 M4 Bttl Fltrt } \\
\text { ¿ J2 208 M4 }\end{array}$ & $\begin{array}{c}4.3 \\
640 \\
51\end{array}$ & $\begin{array}{l}1.5 \\
72 \\
23\end{array}$ & $\begin{array}{l}750 \\
25.769 \\
29.1013\end{array}$ & $\begin{array}{c}3.2 \\
16 \\
2.1\end{array}$ & $\begin{array}{l}1.1 \\
1.9 \\
0.94\end{array}$ & 22 & 4 & 29 & 5.3 & 8.7 & 10. & -0.038 \\
\hline $\begin{array}{l}\text { J2 } 213 \text { M4 Fluid } \\
\text { J2 } 213 \text { M4 Dregs } \\
\text { J2 } 213 \text { M4 Bttl Fltrt } \\
\text { ¿ J2 } 213 \text { M4 }\end{array}$ & $\begin{array}{c}3.7 \\
180 \\
49\end{array}$ & $\begin{array}{l}0.65 \\
10.8 \\
12.7\end{array}$ & $\begin{array}{l}750 \\
111.933 \\
28.4873\end{array}$ & $\begin{array}{c}2.8 \\
20 . \\
1.8\end{array}$ & $\begin{array}{l}0.48 \\
1.21 \\
0.470\end{array}$ & 25 & 2 & 33 & 2.9 & 6.2 & 19 & -0.92 \\
\hline 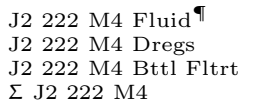 & $\begin{array}{c}14.9 \\
1,730 \\
111\end{array}$ & $\begin{array}{l}0.82 \\
26 \\
12.5\end{array}$ & $\begin{array}{l}750 \\
20.93 \\
28.9633\end{array}$ & $\begin{array}{c}11.2 \\
36.2 \\
3.82\end{array}$ & $\begin{array}{l}0.61 \\
0.54 \\
0.432\end{array}$ & 51 & 2 & 68 & 2.1 & 4.6 & 58 & -0.69 \\
\hline $\mathrm{J} 2228 \mathrm{M} 2 \mathrm{Bttm} \mathrm{H}_{2} \mathrm{O}$ & 117.5 & 33 & & & & & & & & 54.0 & & \\
\hline
\end{tabular}

* For fluid and dregs subcomponents, total Mo and Re inventories are calculated by multiplying the concentrations and solution masses, but because the bottle filtrate fractions were not obtained from filtering the entire $750 \mathrm{~g}$ of fluid, the following scaling factors are applied to the bottle filtrate solution masses (the solution mass filtered is the denominator):

$\mathrm{J} 2208 \mathrm{M} 2:$ factor $=750 \mathrm{~g} / 389.52 \mathrm{~g}=1.9255$

J2 $208 \mathrm{M} 4:$ factor $=750 \mathrm{~g} / 539.16 \mathrm{~g}=1.3911$

$\mathrm{J} 2213 \mathrm{M} 2:$ factor $=750 \mathrm{~g} / 578.27 \mathrm{~g}=1.2970$

Calculated assuming an initial solution mass of 750

Calculated assuming an initial solution mass of $750 \mathrm{~g}$.

S Calculated a

ब Fluid samples were collected in August and September 2006 at approximately $-3.7225^{\circ} \mathrm{S}, 151.6750^{\circ} \mathrm{W}$. Temperature and $\mathrm{pH}$ (recalculated to $\left.25^{\circ} \mathrm{C}\right)$ listed below are from CRADDOCK (2008).

$\begin{array}{llll}\text { J2 } 208 \mathrm{M} 4: & \mathrm{T}=272^{\circ} \mathrm{C} & \mathrm{pH}=2.7 \\ \mathrm{~J} 2 & 208 \mathrm{M} 2: & \mathrm{T}=314^{\circ} \mathrm{C} & \mathrm{pH}=2.4 \\ \mathrm{~J} 2 & 2084 & \mathrm{~T}=278^{\circ} \mathrm{C} & \mathrm{pH}\end{array}$

$\mathrm{J} 2208 \mathrm{M} 4: \quad \mathrm{T}=278^{\circ} \mathrm{C} \quad \mathrm{pH}=2.5$

J2 $213 \mathrm{M} 4: \quad \mathrm{T}=341^{\circ} \mathrm{C} \quad \mathrm{pH}=2.6$

Table 2.6: Re, Mo, and $\mathrm{Mg}$ chemical data for four hydrothermal fluid samples from the Roman Ruins vent site, PACMANUS, Manus Basin. 


\subsection{Discussion}

\subsubsection{Complexities in determining "global" river character- istics}

Studies attempting to describe average river composition at the global scale must, of necessity, incorporate several assumptions. Chief among these is that the river subset being studied is representative of the global average. The total global river $\mathrm{H}_{2} \mathrm{O}$ flux of $3.86 \times 10^{16} \mathrm{Lyr}^{-1}$ (FEKETE ET AL., 2002) is composed of thousands of rivers and streams making it essentially impossible to fully characterize. Figure 2-2 shows the increasing cumulative $\mathrm{H}_{2} \mathrm{O}$ flux (as a proportion of the total) with the increasing number of rivers considered, where the rivers are ranked according to descending discharge (Amazon, Congo, Orinoco, Yangtze ... ); flux data are taken from MEYBEck AND RAGU (1995) and references therein. The exponential fit to the data describes the "diminishing returns" from sampling successively smaller rivers, though it also indicates large proportions of the global flux can be obtained with a "convenient" number of samples. However, precautions must be taken to minimize biases imposed by large individual rivers (see Section 2.5.2).

It has been proposed that when $>50 \%$ of global water-fluxes (or drainage areas) are accounted for, direct extrapolation of flux-weighted averages to the globe is appropriate (MEybeck And Helmer, 1989). Using the data of MEYBECK AND Ragu (1995), this is achieved by sampling the 28 (water flux) or 296 (drainage area) largest rivers. If $<50 \%$ of water flux or continental area are accounted for, extrapolation is done to some set of sub-regions, such as the continents (Livingstone, 1963; Berner AND BERnER, 1987) or distinctive morphoclimatic regions (MEYBECK, 1979). These regional values are then summed to account for global runoff. Rivers should be sam-

pled in an attempt to capture the large inter-river variability caused by differences in basin morphology, climate, and geology.

As there is also significant intra-river variability, one sampling event represents a 

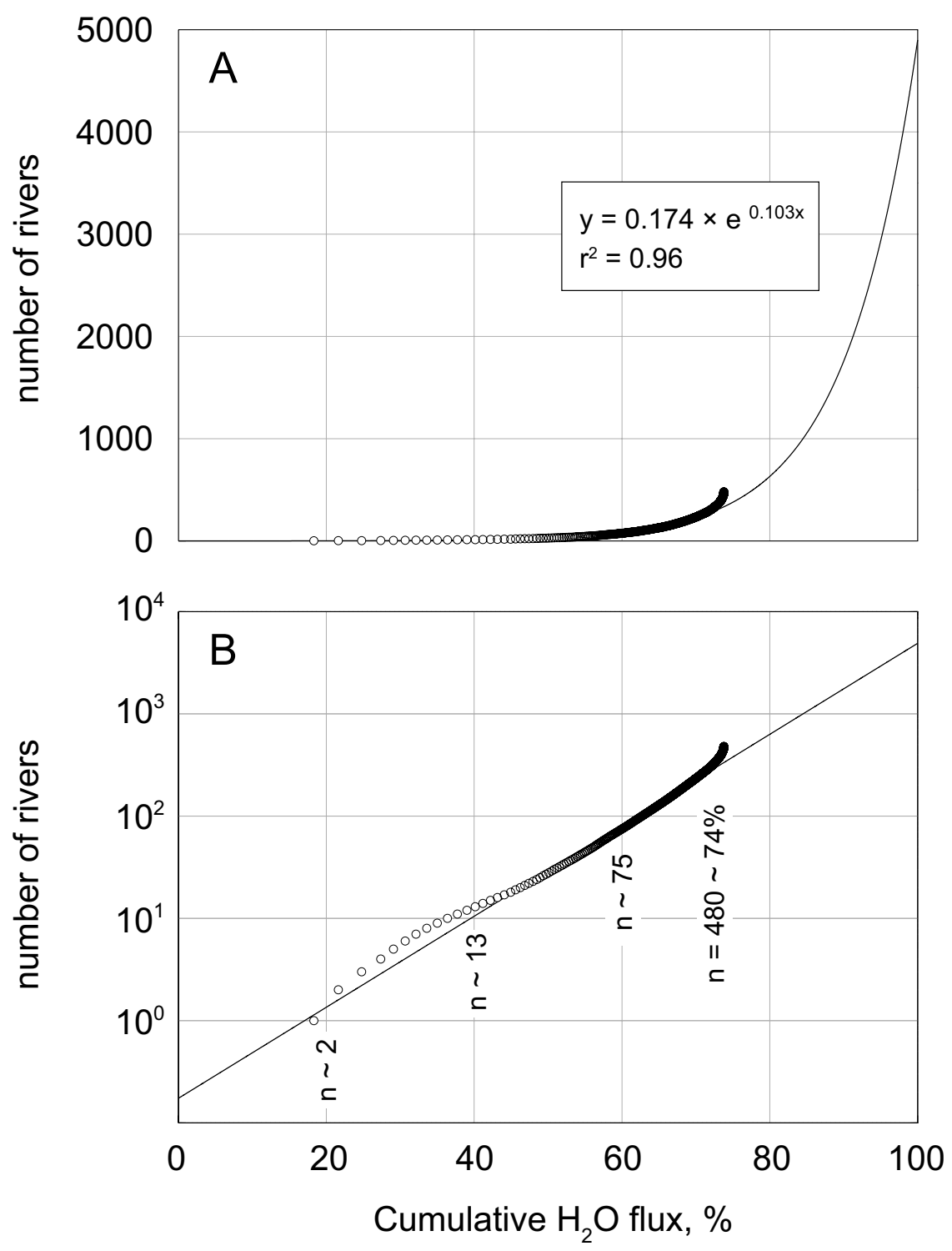

Figure 2-2: Number of rivers and cumulative water flux for world rivers. Water flux data are from MEYBECK AND RAGU (1995, and references therein). Approximately 480 rivers are ranked in order of decreasing water flux with increasing total number of rivers plotted against the proportion of the total river flux. Model fit is exponential $-y=0.174 \times$ $\exp ^{0.103 x}$.
A: Linear y axis
B: $\log _{10}$ y axis. 
single snapshot of the river in time and space. Attempts to determine a time-space integration of the river should therefore be attempted wherever possible. Obtaining spatially integrated samples is theoretically simple; samples obtained at the river mouth are representative of the entire drainage region, while those sampled upstream do not account for some portion of the watershed (related to distance from the mouth and the locations of major tributaries). Unfortunately, temporal averages are significantly more labor intensive. Truly reliable averages for a single river should be obtained with time-series measurements that are then flux weighted to give a yearly average (Meybeck And Helmer, 1989; Livingstone, 1963). Though this is desirable, the number of rivers required to provide large proportions of total water flux (Fig. 2-2) renders this prohibitive or at the least very costly.

In many important cases, even monthly surveys may not be sufficient (LiVINGSTONE, 1963). Figure 2-3 A shows the 2004 water flux for the Yenisei River (see also Table 2.1). The large $\mathrm{H}_{2} \mathrm{O}$ pulse associated with the spring freshet accounts for $\sim 40 \%$ of the yearly $\mathrm{H}_{2} \mathrm{O}$ flux over the course of one month ( $\sim 8 \%$ of yearly time). In the case of large Arctic rivers such as the Yenisei, the freshet must be sampled in order to estimate the yearly total.

Because of the difficulty of obtaining river samples for specific locations and times, many samples are samples of opportunity. For example, significant tributaries may be assumed to represent the main stem of a river. Figure 2-4 A is a comparison of the sum of 2004 daily $\mathrm{H}_{2} \mathrm{O}$ fluxes from four major Mississippi tributaries (the Ohio, Upper Mississippi, Missouri, and Arkansas Rivers) with the true Mississippi $\mathrm{H}_{2} \mathrm{O}$ flux (taken from the U. S. Army Corps of Engineers monitoring station at Tarbert Landing, MS). A stacked area diagram of the proportions of the tributary fluxes is presented in Figure 2-4 B. The sum of the four tributary $\mathrm{H}_{2} \mathrm{O}$ fluxes is representative of the Mississippi $\mathrm{H}_{2} \mathrm{O}$ flux in terms of both magnitude $\left(554 \mathrm{~km}^{3} \mathrm{yr}^{-1}\right.$ and $521 \mathrm{~km}^{3}$ $\mathrm{yr}^{-1}$ respectively) and the location of individual $\mathrm{H}_{2} \mathrm{O}$ flux maxima. Consideration of Figure 2-4 B suggests that the Ohio River alone might also be representative of the 


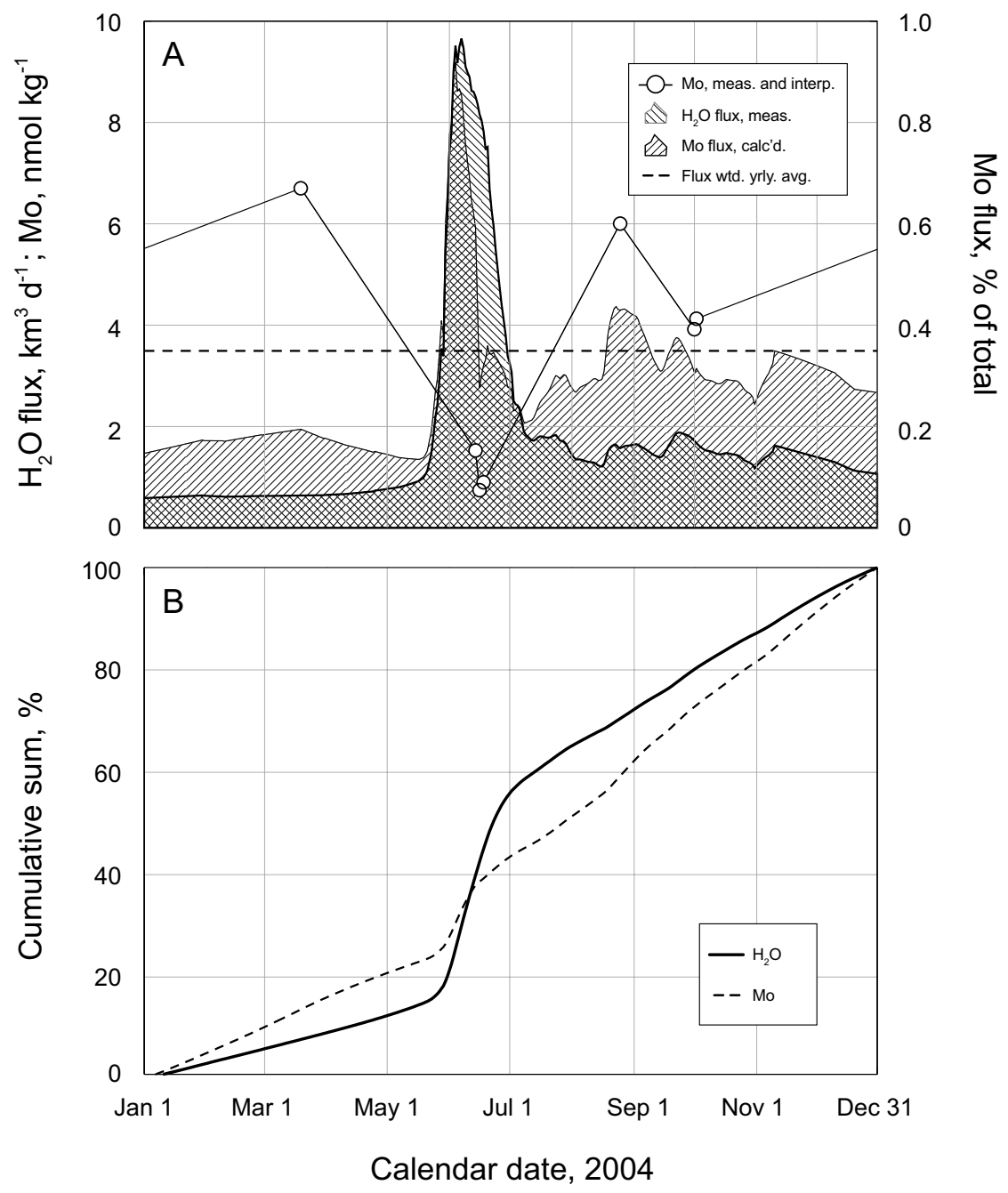

Figure 2-3: Fluxes of $\mathrm{H}_{2} \mathrm{O}$ and Mo, as well as Mo concentrations for the Yenisei River, 2004.

A: Water flux data are from http://rims.unh.edu/ (see footnotes Table 2.1). Intervals between Mo measurements are interpolated linearly (point-to-point). Daily Mo fluxes are the product of daily $\mathrm{H}_{2} \mathrm{O}$ fluxes and interpolated or measured Mo concentrations. These daily fluxes are summed and divided by the yearly $\mathrm{H}_{2} \mathrm{O}$ flux to determine flux-weighted yearly average Mo concentration.

B: Cumulative Yenisei $\mathrm{H}_{2} \mathrm{O}$ and Mo fluxes for 2004. 

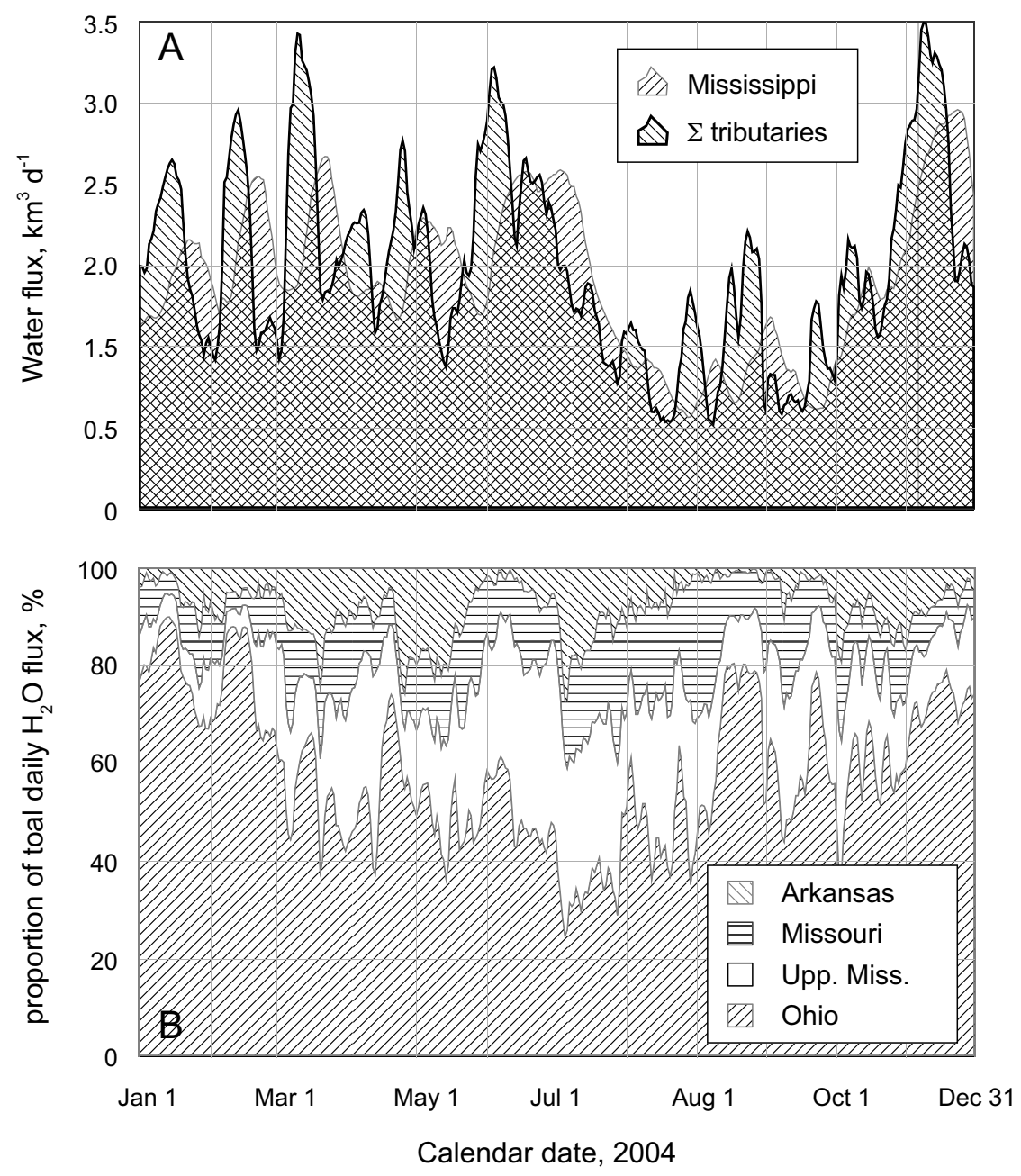

Figure 2-4: Water fluxes of the Mississippi River and its major tributaries: the Ohio, Upper Mississippi, Missouri and Arkansas rivers.

A: Daily $\mathrm{H}_{2} \mathrm{O}$ fluxes for the Mississippi and the sum of tributaries. Data are taken from http://waterdata.usgs.gov/nwis/ (see footnotes Table 2.1). Note the good agreement between the local flux maxima. The slight temporal offset represents the travel time between tributary confluences to the Mississippi at Tarbert Landing, MS. From these data, we calculate yearly $\mathrm{H}_{2} \mathrm{O}$ fluxes of $521 \mathrm{~km}^{3} \mathrm{yr}^{-1}$ for the Mississippi and $554 \mathrm{~km}^{3} \mathrm{yr}^{-1}$ for the sum of the main tributaries. The sum of the four major Mississippi River tributaries is a good analogue for the Mississippi as a whole.

B: Proportions of the daily $\Sigma_{\text {tribs }} \mathrm{H}_{2} \mathrm{O}$ flux for the Ohio, Upper Mississippi, Missouri, and Arkansas Rivers. 
Mississippi in terms of $\mathrm{H}_{2} \mathrm{O}$ flux variations.

For each of the four major Mississippi River tributaries, chemical concentrations were determined for four samples from February, April, July, and November. The samples provide the basis for linear interpolations used to calculated daily chemical concentrations for each tributary. In turn these daily concentrations are multiplied by daily measured $\mathrm{H}_{2} \mathrm{O}$ fluxes to obtain daily chemical fluxes. Finally, these chemical fluxes are summed across all four tributaries to represent the Mississippi River. This data allows us to compare these tributaries and evaluate how representative each is for the Mississippi as a whole; this is done for Re in Figure 2-5. The $\mathrm{x}$-axis denotes the calculated Re concentration for the whole Mississippi (sum of all four tributaries) while the y-axis indicates the contributions of each tributary to the total Re flux, for both measured (large open circles) and interpolated (smaller solid circles) data; stippled lines are the best linear fit to all the data. Where one tributary was perfectly representative of the total river flux, we would expect to see a perfect correlation between the proportional Re flux of that tributary and the calculated Re concentration for the whole Mississippi as a whole (Figure 2-5, abscissa). The proportional Re contributions of both the Upper Mississippi and Ohio rivers can be used to broadly reconstruct the Mississippi concentration as a whole, while those of the Missouri and Arkansas rivers cannot.

In this study only one of the exorheic rivers presented in Table 2.2 has been represented by a major tributary (the Tinguiririca is assumed to represent the Rapel).

\subsubsection{Calculation of present-day average Mo and Re concen- trations in rivers}

Initial calculation of the world river averages of Mo and Re used sample subsets of three rivers (Amazon, Congo, Maipo) and four rivers (Amazon, Orinoco, Ganges, Brahmaputra), respectively (Bertine And Turekian, 1973; Colodner et AL., 1993a). Using global flux and exorheic land area data from FEKETE ET AL. (2002) 

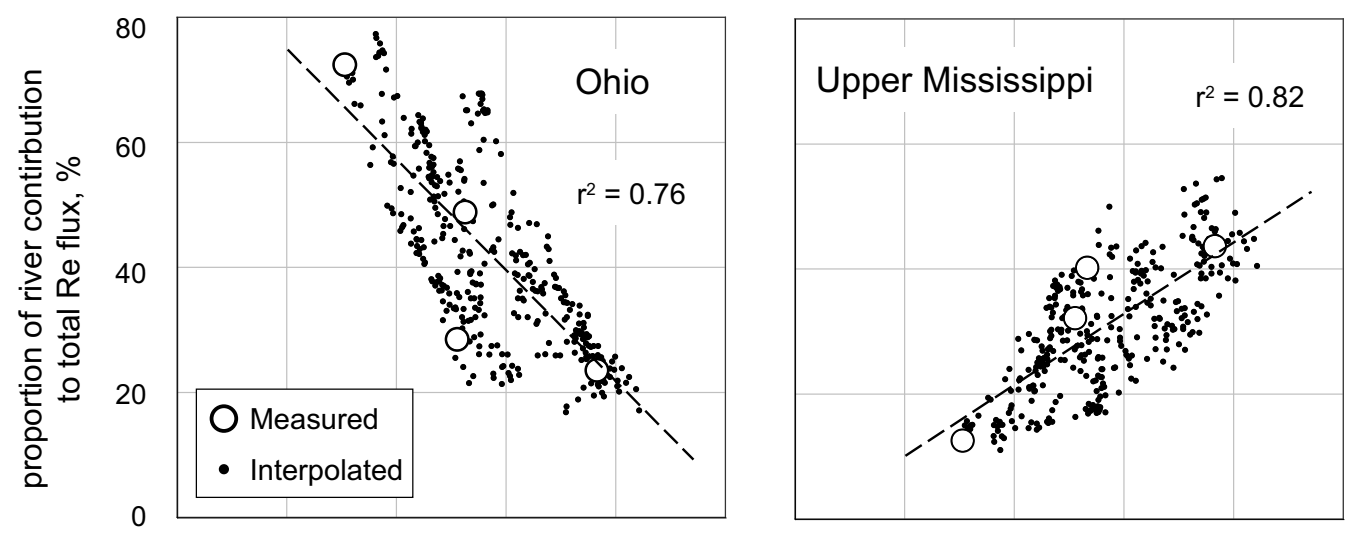

80
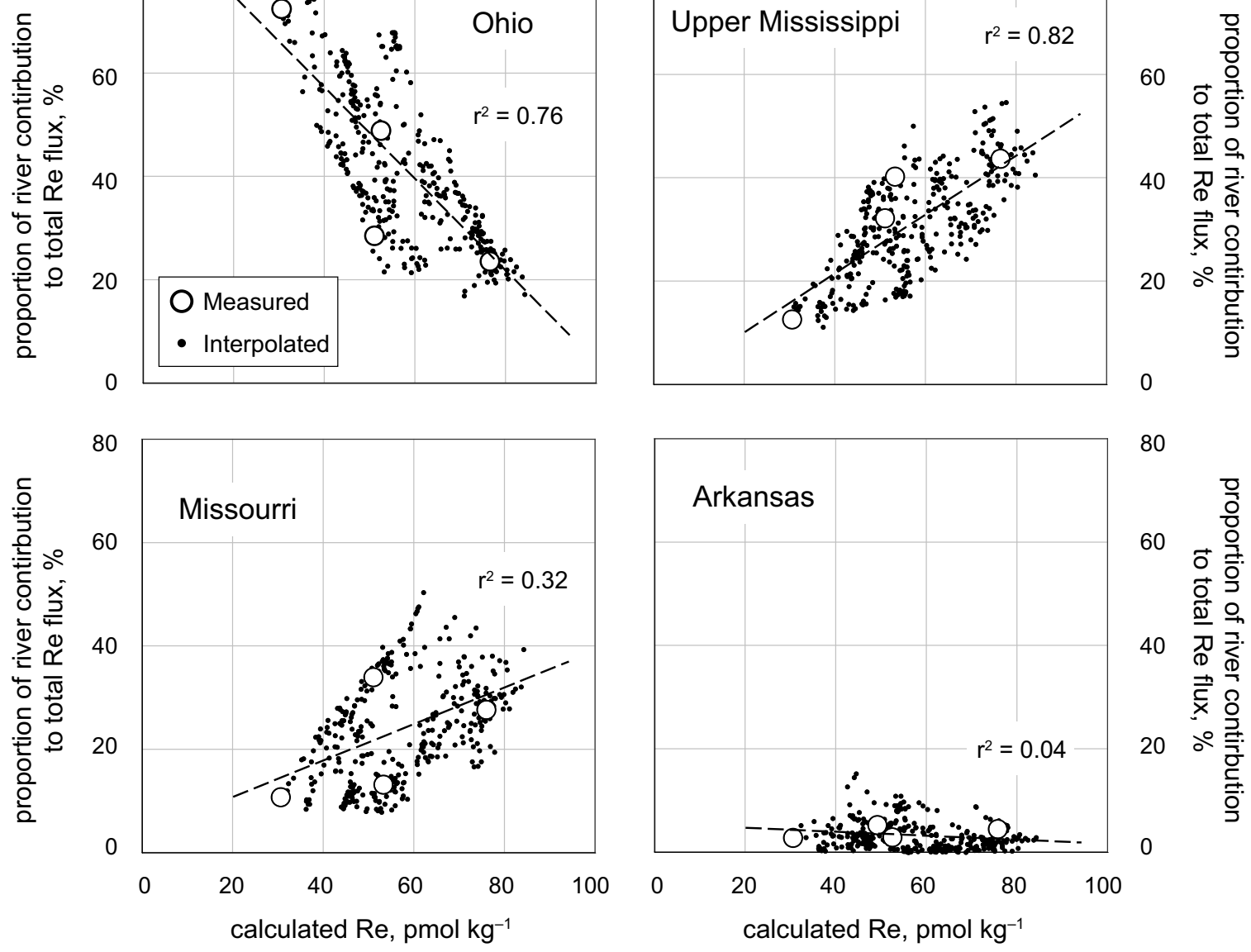

Figure 2-5: Proportion of daily calculated Re concentration vs. daily calculated Re flux for each of the major Mississippi tributaries as a means of assessing proportional tributary impact on the calculated Mississippi Re concentration. The Arkansas River affects the Re concentration very little, while the Missouri does so only moderately. The two rivers with the largest influence on calculated Re concentration are the Upper Mississippi and the Ohio. 
and Peucker-Ehrenbrink and Miller (2007), these sample subsets account for $22 \%(\mathrm{Mo})$ and $24 \%$ (Re) of the water flux and $7.6 \%$ (Mo) and $6.6 \%$ (Re) of exorheic continental area. Such small sample sets would seemingly necessitate extrapolation according to continent or morphoclimatic zone areas, but such attempts would be meaningless as so few of these regions are characterized. As a result, these early investigations extrapolated directly to the global water fluxes.

This study analyses a sub-sample set of 38 exorheic rivers (Table 2.2), encompassing $37 \%$ of total water runoff and $22 \%$ of total exorheic drainage area. Published and calculated world river averages of some major elements are compared in Table 2.8. Using the data of MEYBECK AND RAGU (1995) and this study, world river averages are calculated by scaling directly to the global $\mathrm{H}_{2} \mathrm{O}$ flux, scaling to continental area, and scaling to Graham drainage region (Graham ET AL., 1999). We have elected to use the 19 drainage regions of GRAHAM ET AL. (1999) rather than the 14 morphoclimatic zones of MEYBECK (1979). Graham drainage regions are delineated according to continental runoff into specific regions of the world ocean and into large inland seas. These regions likely represent the most informative subdivision of continental area both because they form part of a river transport algorithm designed to be used in climate modeling (GrahAm ET AL., 1999), and especially because they have been characterized in terms of bedrock geology (Peucker-Ehrenbrink And Miller, 2007) potentially illustrating the effects of lithology on the dissolved river load. Table 2.5.2 lists the Graham drainage areas for which data are presented in this study, and provides a description of how chemical averages are obtained for both the global average and individual Graham regions.

The data compilation of MEYBECK AND RAGU (1995) contains major ion data for approximately 250 rivers accounting for $\sim 60 \%$ and $\sim 51 \%$ of respective global $\mathrm{H}_{2} \mathrm{O}$ flux and drainage area. World river averages calculated using the MEYBECK AND RAGU (1995) compilation agree well with the values of MEYBECK (1979) and Livingstone (1963). Calculated MEyBEck AND RAGU (1995) averages also agree well across the 
Graham regional chemical concentration averages

\begin{tabular}{|c|c|c|c|c|c|c|c|c|c|c|}
\hline $\begin{array}{c}\text { Graham } \\
\text { Region }\end{array}$ & $\begin{array}{r}\begin{array}{r}f \mathrm{H}_{2} \mathrm{O} \\
\mathrm{km}^{3} \mathrm{yr}^{-1}\end{array}\end{array}$ & $\begin{array}{r}f \mathrm{H}_{2} \mathrm{O}, * \\
\text { prop. }\end{array}$ & $\begin{array}{r}\mathrm{Re}^{\ddagger} \neq \\
\text { pmol kg }\end{array}$ & $\begin{array}{r}\mathrm{Moo}^{\ddagger} \\
\mathrm{nmol} \mathrm{kg}{ }^{-1}\end{array}$ & 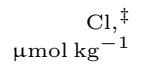 & $\begin{array}{r}\mathrm{SO}_{4},{ }^{\ddagger} \\
\mu \mathrm{mol} \mathrm{kg}{ }^{-1}\end{array}$ & $\begin{aligned} \mathrm{Na} & \neq \\
\mu \mathrm{mol} \mathrm{kg} & -1\end{aligned}$ & $\begin{array}{r}\mathrm{Mg}_{,}^{\ddagger} \\
\mu \mathrm{mol} \mathrm{kg}{ }^{-1}\end{array}$ & $\begin{array}{r}\mathrm{Ca}{ }^{\ddagger} \\
\mu \mathrm{mol} \mathrm{kg}{ }^{-1}\end{array}$ & $\begin{array}{r}\mathrm{K}, \ddagger \\
\mu \mathrm{mol} \mathrm{kg}\end{array}$ \\
\hline 1 & 2855.8 & 0.082 & 6.5 & 3.3 & 182.1 & 84.6 & 266.7 & 155.3 & 383.4 & 16.5 \\
\hline 2 & 846.1 & 0.024 & 16.2 & 10.1 & 239.8 & 378.4 & 352.9 & 358.5 & 870.8 & 22.9 \\
\hline 3 & 2687.8 & 0.077 & 40.6 & 16.5 & 603.4 & 376.4 & 498.3 & 281.6 & 777.3 & 34.0 \\
\hline 4 & 821.7 & 0.024 & 42.0 & 8.5 & 908.9 & 297.1 & 670.9 & 274.2 & 1111.9 & 59.5 \\
\hline 5 & 10898.6 & 0.313 & 1.8 & 0.9 & 8807.5 & 91.5 & 99.6 & 67.8 & 164.5 & 29.6 \\
\hline 6 & 2426.9 & 0.070 & 3.3 & 0.7 & 62.5 & 29.1 & 120.6 & 71.8 & 61.6 & 39.0 \\
\hline 8 & 4050.7 & 0.116 & 5.1 & 11.3 & 95.1 & 133.6 & 216.6 & 212.6 & 604.9 & 66.8 \\
\hline 9 & 7001.3 & 0.201 & 42.1 & 17.0 & 3041.5 & 331.2 & 411.9 & 314.1 & 692.4 & 41.1 \\
\hline 10 & 1688.3 & 0.049 & 10.4 & 10.9 & 49.4 & 216.6 & 112.6 & 222.7 & 640.5 & 31.0 \\
\hline \multirow{3}{*}{$\begin{array}{l}11 \\
16\end{array}$} & 1128.5 & 0.032 & 7.0 & 8.4 & 204.5 & 203.4 & 383.3 & 133.3 & 347.8 & 29.2 \\
\hline & 403.5 & 0.012 & 74.3 & 10.8 & 1832.4 & 1032.4 & 2024.3 & 1196.4 & 1853.1 & 161.6 \\
\hline & & $\Sigma$ & $16.5^{\dagger}$ & $8.0^{\dagger}$ & $746.3^{\dagger}$ & $194.1^{\dagger}$ & $273.8^{\dagger}$ & $192.8^{\dagger}$ & $468.2^{\dagger}$ & $38.3^{\dagger}$ \\
\hline
\end{tabular}

* Proportions of $\mathrm{H}_{2} \mathrm{O}$ fluxes are simply the individual regional fluxes divided by the sum $\left(34,809 \mathrm{~km}^{3} \mathrm{yr}^{-1}\right)$. Note that though we do not have samples for Graham Regions

The global average of a chemical species is obtained by multiplying the regional species concentration by the the regional $\mathrm{H}_{2} \mathrm{O}$ flux proportion for all

and then summing the results.

(ne in a region are then summed.

multiplied by the proportion of the regional $\mathrm{H}_{2} \mathrm{O}$ flux represented by that river. The values from all rivers in a region are then summed.

Table 2.7: Water fluxes, water flux proportions, and average regional chemical concentrations for all Graham drainage regions for which data are presented in this study. Calculated world river chemical concentrations are also shown. 


\begin{tabular}{|c|c|c|c|c|c|c|c|c|}
\hline \multirow{2}{*}{$\begin{array}{l}\text { A } \\
\text { Source } \\
\text { Calc. method }\end{array}$} & \multirow{2}{*}{$\begin{array}{c}\text { Published: } \\
\text { MEYBECK (1979) } \\
\text { Scaled to area, } \\
\text { Morpho-climatic zone }\end{array}$} & \multirow{2}{*}{$\begin{array}{l}\text { Published: } \\
\text { LivingSTONE (1963) } \\
\text { Scaled to area, } \\
\text { Continents }\end{array}$} & \multicolumn{3}{|c|}{$\begin{array}{l}\text { Calculated: } \\
\text { data from MEYBECK AND RAGU (1995) }\end{array}$} & \multicolumn{3}{|c|}{$\begin{array}{l}\text { Calculated: } \\
\text { data from this study, see Table } 2.2\end{array}$} \\
\hline & & & $\begin{array}{l}\text { Scaled to global } \\
\mathrm{H}_{2} \mathrm{O} \text { flux }\end{array}$ & $\begin{array}{l}\text { Scaled to area, } \\
\text { Continents }\end{array}$ & $\begin{array}{l}\text { Scaled to area } \\
\text { Graham Region }\end{array}$ & $\begin{array}{l}\text { Scaled to global } \\
\mathrm{H}_{2} \mathrm{O} \text { flux }\end{array}$ & $\begin{array}{l}\text { Scaled to area, } \\
\text { Continents }\end{array}$ & $\begin{array}{l}\text { Scaled to area, } \\
\text { Graham Region }\end{array}$ \\
\hline $\mathrm{Cl}, \mu \mathrm{mol} \mathrm{kg}{ }^{-1}$ & 230 & 220 & 240 & 270 & 280 & 400 & 920 & 750 \\
\hline $\mathrm{SO}_{4}, \mu \mathrm{mol} \mathrm{kg}{ }^{-1}$ & 120 & 117 & 117 & 126 & 137 & 156 & 206 & 194 \\
\hline $\mathrm{Na}, \mu \mathrm{mol} \mathrm{kg}-1$ & 310 & 270 & 280 & 310 & 330 & 210 & 330 & 270 \\
\hline $\mathrm{Mg}, \mu \mathrm{mol} \mathrm{kg}^{-1}$ & 150 & 170 & 160 & 180 & 180 & 150 & 220 & 190 \\
\hline $\mathrm{Ca}, \mu \mathrm{mol} \mathrm{kg}{ }^{-1}$ & 367 & 370 & 370 & 420 & 420 & 350 & 510 & 470 \\
\hline $\mathrm{K}, \mu \mathrm{mol} \mathrm{kg}-1$ & 36 & 59 & 38 & 38 & 67 & 34 & 41 & 38 \\
\hline
\end{tabular}

\begin{tabular}{|c|c|c|c|c|}
\hline \multirow[t]{2}{*}{$\begin{array}{l}\text { B } \\
\text { Source }\end{array}$} & \multirow{2}{*}{$\begin{array}{c}\text { Published: } \\
\text { Mo Bertine AND TUREKIAN (1973) } \\
\text { Re COLODNER ET AL. (1993a) } \\
\text { Scaled to global } \\
\mathrm{H}_{2} \mathrm{O} \text { flux }\end{array}$} & \multicolumn{3}{|c|}{$\begin{array}{c}\text { Calculated: } \\
\text { data from this study, see Table } 2.2\end{array}$} \\
\hline & & $\begin{array}{l}\text { Scaled to global } \\
\mathrm{H}_{2} \mathrm{O} \text { flux }\end{array}$ & $\begin{array}{l}\text { Scaled to area, } \\
\text { Continents }\end{array}$ & $\begin{array}{l}\text { Scaled to area, } \\
\text { Graham Region }\end{array}$ \\
\hline Mo, $\mathrm{nmol} \mathrm{kg}^{-1}$ & 4.5 & 5.5 & 7.6 & 8.0 \\
\hline $\mathrm{Re}, \mathrm{pmol} \mathrm{kg}^{-1}$ & 2.1 & 11.5 & 17.3 & 16.5 \\
\hline
\end{tabular}

Table 2.8: A: Comparison of world river average concentrations of major ions estimated using different data sets and calculated using different methods of extrapolation.

B: Comparison of published world river average concentrations of Mo and Re with those calculated from this study using different methods of extrapolation; none have been corrected for anthropogenic metal contributions. 
different calculation methods for all ions except K. This generally good agreement is to be expected for a data set accounting for such a large proportion of global totals. The same calculations done with the data set presented in Table 2.2 shows good agreement with published values of $\mathrm{Na}, \mathrm{Mg}$, and $\mathrm{K}$, is somewhat higher for $\mathrm{Ca}$ and $\mathrm{SO}_{4}$, and is significantly higher than published values for $\mathrm{Cl}$. As one would expect for a significantly smaller sample set, the calculated data for this study also show larger variations across the different calculation methods. Consideration of the river averages calculated for the major ions indicates that, with the exception of $\mathrm{Cl}$, our sub-sample set is broadly consistent with global river chemistry and can therefore be used to recalculate the world river average concentrations of Mo and Re. The results of these calculations are presented in Table 2.8. Note that with the exception of precipitation samples, data were not corrected for seasalt contributions using chlorinity as this often resulted in negative concentrations for other ions (particularly $\mathrm{SO}_{4}^{2-}$ and $\mathrm{Na}^{+}$). In any case, with the exception of estuarine samples (Table 2.5) seawater contributions of Mo and Re never exceeded $0.1 \%$. Precipitation samples were chloride corrected as the observed $\mathrm{Mg} / \mathrm{Cl}$ ratios of these samples were identical to that of seawater.

Recalculated world river averages of Mo and Re are both significantly higher than original estimates. Assuming that scaling to Graham drainage region is the most appropriate way of calculating average river concentration, then values of Mo and Re are respectively two and eight times greater than previous assessments (BERTINE And Turekian, 1973; Colodner Et Al., 1993a). Such significant changes are not unexpected due to the small numbers of rivers considered for the previous assessments. For Mo, the previous estimate was calculated using the Amazon, Congo, and Maipo rivers (Bertine And Turekian, 1973) while the Amazon, Brahmaputra, Ganges and Orinoco were used for the original calculation of Re (COLODNER ET AL., 1993a). The ranges of Mo and Re concentrations seen in this study (Table 2.2) was not accounted for. Previous averages were calculated by $\mathrm{H}_{2} \mathrm{O}$-flux weighting and scaled directly to the global river flux, the values for Mo and Re are overwhelmingly 
dominated by the low concentrations of these metals in the Amazon. The Amazon river accounts for 76 and $85 \%$ of total $\mathrm{H}_{2} \mathrm{O}$ fluxes used in these previous studies, but represents only $\sim 17 \%$ the global river $\mathrm{H}_{2} \mathrm{O}$ flux. This Amazon bias is also seen in the world river averages calculated according to different methods in this study (Table 2.8). The Amazon represents $\sim 46 \%$ of the $\sim 37 \%$ of the global river $\mathrm{H}_{2} \mathrm{O}$ flux accounted for by the rivers in Table 2.2. Because Mo and Re concentrations are low in the Amazon, averages calculated by simply scaling to the global $\mathrm{H}_{2} \mathrm{O}$ flux are lower than those obtained by first scaling to continental region or Graham region in which the proportional water flux of the Amazon is kept closer to its global value of $\sim 17 \%$.

Using the present-day Mo and Re average river concentrations of $8.0 \mathrm{nmol} \mathrm{kg}^{-1}$ and $16.5 \mathrm{pmol} \mathrm{kg}^{-1}$ along with a global river $\mathrm{H}_{2} \mathrm{O}$ flux of $3.86 \times 10^{16} \mathrm{~kg} \mathrm{yr}^{-1}(\mathrm{FEKETE}$ ET AL., 2002), modern fluxes of these metals to seawater are $3.09 \times 10^{8} \mathrm{molyr}^{-1}$ $(\mathrm{Mo})$ and $6.37 \times 10^{5} \mathrm{molyr}^{-1}(\mathrm{Re})$. It must be noted, however, that these modern fluxes have not been apportioned into anthropogenic and natural fluxes.

\subsubsection{Anthropogenic contributions and the pre-anthropogenic world river average of $\mathrm{Re}$}

While the relatively low crustal values of Mo and Re (MCLEnnAn, 2001) enhance their usefulness as redox indicators, the industrial production and use of these heavy metals introduces an anthropogenic component to their modern surface cycles. The recalculated world river average Mo and Re concentrations presented thus far represent significant increases relative to previous assessments (1.8-fold for Mo, BERTINE And Turekian, 1973; 7.9-fold for Re, Colodner et AL., 1993a). Given that anthropogenic activities are known to influence the surface cycling of other heavy metals (e.g. $\mathrm{Pb}$ ), it is possible that these higher world river average concentrations represent an anthropogenic enhancement. The potential for pollutive contributions of Mo was recognized by BERTINE AND TUREKIAN (1973) and accounts for their decision to determine a world river average Mo concentration based on three rivers from the 
southern hemisphere. Likewise, Evans ET AL. (1978) used analyses of pristine Arctic rivers to estimate that modern Mo fluxes had approximately doubled due to anthropogenic contributions. Similarly, Colodner ET AL. (1995) invoked anthropogenic contamination to explain Re concentrations for rivers draining into the Black Sea that were significantly higher than the world average.

An anthropogenic enhancement of Mo and Re delivery to modern oceans might be expected on the basis of the high concentrations of these metals in fossil fuels (Bertine and Goldberg, 1971; Poplavko et Al., 1974; Duyck et Al., 2002; Selby et AL., 2005, 2007a). Both Mo (Moyse, 2000) and Re (Chang, 1998) are also found in fuel processing catalysts, and the use of Re in brake liners is thought to be the source for high concentrations of Re (up to $10 \mathrm{ng} \mathrm{g}^{-1}$ ) in road dusts (MEISEL AND Stotter, 2007).

Examination of major world rivers (Table 2.2) suggests that human contributions are of Mo and Re are certainly possible. Some rivers that are known to be anthropogenically impacted contain high metal concentrations (Mississippi, Rhine, Yangtze, Danube). However, many rivers considered to be pristine or to show low degrees of anthropogenic influence also exhibit high Mo and (especially) Re (Ob, Mackenzie, St. Lawrence, Fly, Yukon, Copper, Tinguiririca, Maipo).

Table 2.4 contains data for precipitation samples from Falmouth, MA, USA. Many of these samples contain non-negligible metal concentrations, even after correction for seawater (Falmouth is bordered by the ocean on three sides and precipitation samples all exhibited a $\mathrm{Mg} / \mathrm{Cl}$ ratio consistent with seawater). For Mo, these concentrations are consistent though generally lower than the concentrations reported in Japanese rain samples (Sugawara And OkABe, 1961). Though these are the first data for Re in precipitation, the unexpectedly high concentrations of some samples (e.g., 5.9

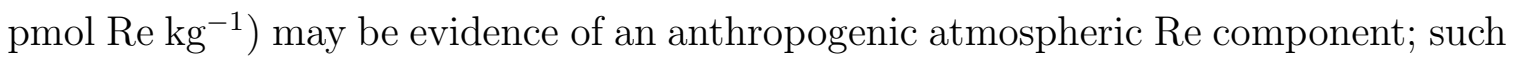
a source has been suggested by CHAPPAZ ET AL. (2008).

Some of the very high Re concentrations for natural waters seen in this study are 
likely the result of anthropogenic contamination. The highest river concentration 1,240 pmol Re $\mathrm{kg}^{-1}$, South Platte River (Table 2.3) — is likely due to evaporative concentration of Re in groundwater used for irrigation; groundwaters can have high concentrations of Re (Hodge et AL., 1996; Leybourne And Cameron, 2008, Fig. 2-7). In addition to precipitation samples, Table 2.4 also contains data for water samples from mining areas such as the Berkeley Pit, MT, USA (a flooded openpit $\mathrm{Cu}$ mine), and the Kupferschiefer near Mansfeld, Germany (a black shale-hosted $\mathrm{Ni}, \mathrm{Cu}, \mathrm{Ag}, \mathrm{Au}, \mathrm{Ag}$, and $\mathrm{Pb}$ mine, KuCHA, 1990). Concentrations of Re in these samples $\left(11,900-37,000 \mathrm{pmol} \mathrm{kg}^{-1}\right)$ are the highest observed for "natural" water samples with enrichment factors of $700-2,200$ times the average river concentration determined in this study. These samples have concentrations equivalent to or higher than estimates of Re in the crust (Esser AND TurEKIAN, 1993; HAURI AND HART, 1997; Sun ET AL., 2003a). Concentrations of Mo in these samples are do not appear to display the same level of enrichment. While the Mansfeld samples show concentrations ranging from 190 - $250 \mathrm{nmol} \mathrm{Mo} \mathrm{kg}^{-1}$ (23 - 32 times world river average), Berkeley Pit samples have Mo concentrations of less than $1 \mathrm{nmol} \mathrm{kg}^{-1}(\sim 0.08$ times the world river average). The extremely high Re enrichments but only low to moderately high Mo enrichments in waters associated with mining suggests that Re may be a particularly sensitive tracer of anthropogenic heavy metal contamination.

Given the compelling evidence for anthropogenic Re contamination (in particular), it must be quantified in order to determine the pre-anthropogenic river average and $\tau_{\text {Re. }}$ The linear relationship observed between Re and $\mathrm{SO}_{4}^{2-}$ (Section 2.5.4, Fig. 2-7) allows us to do this. The anthropogenic $\mathrm{SO}_{4}^{2-}$ component of world rivers is estimated at 28\% (BERNER, 1971; MEYBECK, 1979), 32\% (using $\mathrm{SO}_{4}^{2-}$ concentrations for pristine waters from MEYBECK, 1988) and 43\% (BERnER AND BERnER, 1987). New world river average calculations depend on the choice of average $\mathrm{SO}_{4}^{2-}$ concentration and the choice of anthropogenic $\mathrm{SO}_{4}^{2-}$ proportion. Using anthropogenic $\mathrm{SO}_{4}^{2-}$ estimates from the literature (BERNER, 1971; BERNER AND BERNER, 1987) as well as 
estimates calculated from the values in Table 2.8, and assuming pristine river $\mathrm{SO}_{4}^{2-}$ concentrations (MEYBeCK AND Helmer, 1989, $81.5 \mu \mathrm{mol} \mathrm{SO} \mathrm{SO}^{2-} \mathrm{kg}^{-1}$ ), we obtain pre-anthropogenic world river Re concentrations ranging from $6.5-11.9 \mathrm{pmol} \mathrm{kg}^{-1}$.

Our calculated best-estimate of anthropogenically-corrected river Re concentration is $11.2 \mathrm{pmol} \mathrm{kg}^{-1}$ and $1.2 \times 10^{5} \mathrm{yr}$; this estimate assumes modern $\mathrm{SO}_{4}^{2-}$ and $\mathrm{Re}$ world river average concentrations of $120 \mu \mathrm{mol} \mathrm{kg}^{-1}$ and $16.5 \mathrm{pmol} \mathrm{kg}^{-1}$ MEYBECK, 1979, Section 2.5.2, as well as a a preindustrial $\mathrm{SO}_{4}^{2-}$ estimate of $81.5 \mu \mathrm{mol} \mathrm{kg}{ }^{-1}$, Meybeck and Helmer, 1989. Assuming a global $\mathrm{H}_{2} \mathrm{O}$ flux of $3.86 \times 10^{16} \mathrm{Lyr}^{-1}$ (FeKETE ET AL., 2002) this translates to a natural (pre-anthropogenic) Re river flux of $4.32 \times 10^{5} \mathrm{molyr}^{-1}$.

Without a relationship such as that seen for $\mathrm{Re}$ and $\mathrm{SO}_{4}^{2-}$, correcting for the anthropogenic contribution of Mo is considerably more difficult. While our reassessment of average river Mo $\left(8.0 \mathrm{nmol} \mathrm{kg}{ }^{-1}\right)$ is considerably higher than the previous

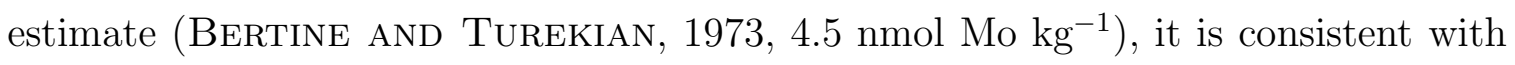
the value of $9.1 \mathrm{pmol} \mathrm{kg}^{-1}$ calculated by Evans ET AL. (1978). Evans ET AL. (1978) estimated a pollution-corrected average of 5 pmol $\mathrm{Mo} \mathrm{kg}^{-1}$ on the assumption that Soviet rivers represented the pre-industrial world river average. If our recalculated Mo river concentration data incorporate a significant ( $50 \%)$ anthropogenic component, the pollution-corrected world river Re average is still significantly larger than previously estimated.

\subsubsection{Continental sources of Mo and Re}

Upper continental crustal concentrations for Mo and Re are $16 \mathrm{nmolg}^{-1}$ and $2 \mathrm{pmol}$ $\mathrm{g}^{-1}$ respectively (McLennan, 2001). In particular, Re is present at the lowest levels of all stable elements save Os and Ir. This low concentration and the rarity with which it forms its own minerals resulted in Re being the last chemical element "discovered" (NodDACK ET AL., 1925; NodDACK AND NodDACK, 1931). A general association between Re and Mo has been recognized ever since this discovery, as Re was first 
isolated from molybdenite $\left(\mathrm{MoS}_{2} ;\right.$ NodDACK ET AL., 1925). A theoretical basis for the association of Mo and Re was later provided by similar ionic radii in their geochemically common oxidation states (Table 2.9),

\begin{tabular}{lccc}
\hline Element & $\begin{array}{c}\text { Oxidation } \\
\text { state }\end{array}$ & $\begin{array}{c}\text { Co-ordination } \\
\text { number }\end{array}$ & $\begin{array}{c}\text { Ionic radius, } \\
\AA\end{array}$ \\
\hline Mo & VI & 5 & 0.58 \\
Re & VII & 6 & 0.65 \\
Mo & IV & 4 & 0.73 \\
Re & IV & 4 & 0.71 \\
\hline
\end{tabular}

Table 2.9: Ionic radii of the most common valences and coordination numbers of Mo and Re; data are from Whittaker And Muntus (1970)

and has been recently confirmed by synchrotron studies of Re and ${ }^{187} \mathrm{Os}$ in $\mathrm{MoS}_{2}$ (TAKAHASHI ET AL., 2007).

Molybdenum commonly occurs as the sulfide ore mineral $\mathrm{MoS}_{2}$ though it also forms various oxomolybdate species $\left(\mathrm{PbMoO}_{4}, \mathrm{CaMoO}_{4}\right.$; Evans ET AL., 1978), and exhibits high concentrations in common accessory minerals such as magnetite and sphene. In contrast, with the exception of one documented oxide occurrence $\left(\mathrm{Re}_{2} \mathrm{O}_{7}\right.$, Peterson ET AL., 1959), Re is associated exclusively with sulphide minerals, either as an accessory or trace element $\left(\mathrm{FeS}_{2}, \mathrm{CuFeS}_{2}, \mathrm{AsFeS}_{2}, \mathrm{MoS}_{2}\right.$; e.g. Fleischer, 1959; Stein ET AL., 1998; Mathur et AL., 2005; Morelli ET AL., 2005) or, very rarely, as an actual Re mineral $\left(\mathrm{ReS}_{2}, \mathrm{ReS}_{3}, \sim \mathrm{CuReS}_{4}, \sim \mathrm{Re}_{4} \mathrm{Mo}_{2} \mathrm{CuFeS}_{11} ;\right.$ CAPITANT ET AL., 1963; Fleischer, 1963; Morris And Short, 1966; Volborth ET AL., 1986; Mitchell et Al., 1989; Korzhinski et Al., 1994; Power et AL., 2004). Aside from sulfides, Mo and Re are known to be enriched in modern and ancient reducing sediments such as black shales (e.g. KoIDE ET AL., 1986; RAVIZZA ET AL., 1991; Crusius et Al., 1996; Morford And Emerson, 1999; Algeo And Lyons, 2006). Within these lithologies, Mo is typically enriched in the diagenetic pyrite 
relative to the host sediment (RAISWELL AND PLANT, 1980), whereas Re is present at higher concentrations in the sediments than associated pyrite (COHEN ET AL., 1999). The solvent-extractability of these metals from organic-rich lithologies indicates that a significant proportion of Re (but not Mo) is organically bound (MILLER, 2004).

The association of Re, Mo, and S in the crust and the high solubilities of their oxidized species $\mathrm{MoO}_{4}^{2-}, \mathrm{ReO}_{4}^{-}$, and $\mathrm{SO}_{4}^{2-}$ implies the effective mobilization of these elements by oxidative weathering (seen for Re in PEUCKER-EHRENBRINK AND HANNIGAn, 2000; JAfFe ET AL., 2002; Pierson-WiCkMAnn ET AL., 2003), and suggests an association in the dissolved phase. While a relationship between dissolved Re and $\mathrm{SO}_{4}^{2-}$ has been observed (COLODNER ET AL., 1993a; DALAi ET AL., 2002), no similar relationship with Mo has been reported. Figures 2-6 and 2-7 show the relationship between dissolved $\mathrm{Mo}, \mathrm{Re}$, and $\mathrm{SO}_{4}^{2-}$ for the waters analysed in this study. The association between Re and Mo, previously observed at the level of a drainage basin (Colodner et AL., 1993a; Dalai ET AL., 2002) can now be extended globally $\left(\mathrm{r}^{2}\right.$ $=0.72)$. No equivalent relationship is seen between $\mathrm{Mo}$ and $\mathrm{SO}_{4}^{2-}\left(\mathrm{r}^{2}=0.11\right)$.

The correlation of $\mathrm{Re}$ and $\mathrm{SO}_{4}^{2-}$ in river and precipitation samples analyzed in this study extends across four orders of magnitude (Fig. 2-7). The observed relationship is consistent with Re and S concentrations for the upper continental crust (UCC), reported groundwater data, and the hypothesized sulphide and black shale sources of Re. Given the high solubilities of Mo, Re, and S as oxic species and the predominance of Mo as a sulphide in the crust, the lack of an equivalent relationship between $\mathrm{Mo}$ and $\mathrm{SO}_{4}^{2-}$ is perhaps somewhat unexpected. However, Mo is known to adsorb to Mn-oxides, whereas Re does not (Bertine And Turekian, 1973; Evans ET AL., 1978; KoIde ET AL., 1986; Siebert ET AL., 2003, see also Chap. 4). In addition, altered molybdenites show decreased Re concentrations (NEWBERRY, 1979; MCCANDLESS ET AL., 1993) while oxic weathering of $\mathrm{MoS}_{2}$ preferentially releases Re (Morachevskit and Nechaeva, 1960), indicating that $\mathrm{MoS}_{2}$ weathering is not perfectly congruent. In light of these observations, and the greater variability of Mo- 


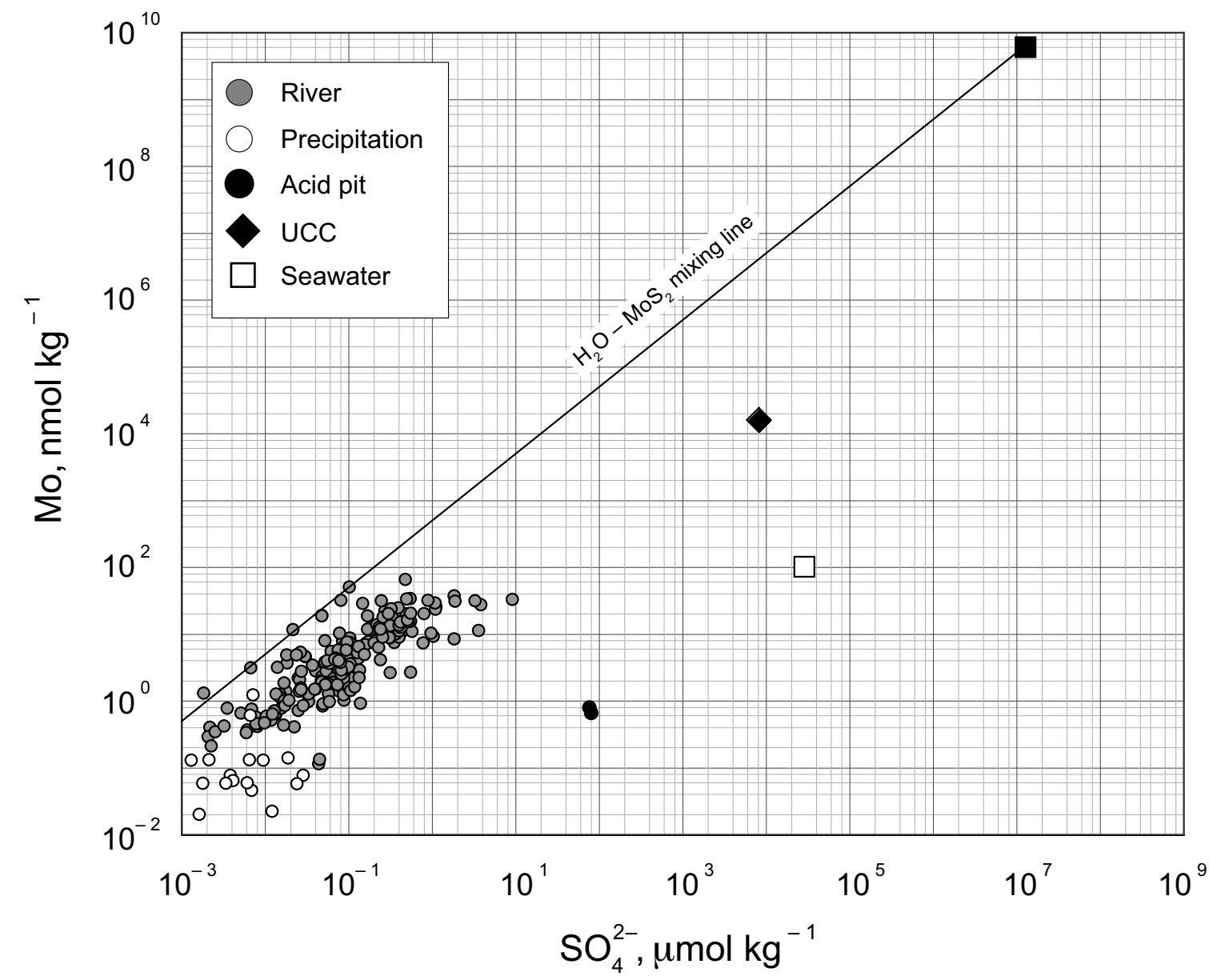

Figure 2-6: Molybdenum and $\mathrm{SO}_{4}^{2-}$ in surface waters, crustal rocks, $\mathrm{MoS}_{2}$, and seawater.

Also plotted is a mixing line between $\mathrm{MoS}_{2}$ and a hypothetical "pure" water (zero- $\mathrm{SO}_{4}^{2-}$, zero-Mo).

$\mathrm{UCC}=$ upper continental crust, Precipitation $=$ rain and snow collected in Falmouth, MA, USA between 2004/09/14 and 2005/09/29, Acid pit = samples of Berkeley Pit open pit mine waters, Butte, MN, USA.

Data for UCC from Mason And Moore (1982); MCLennan (2001). 


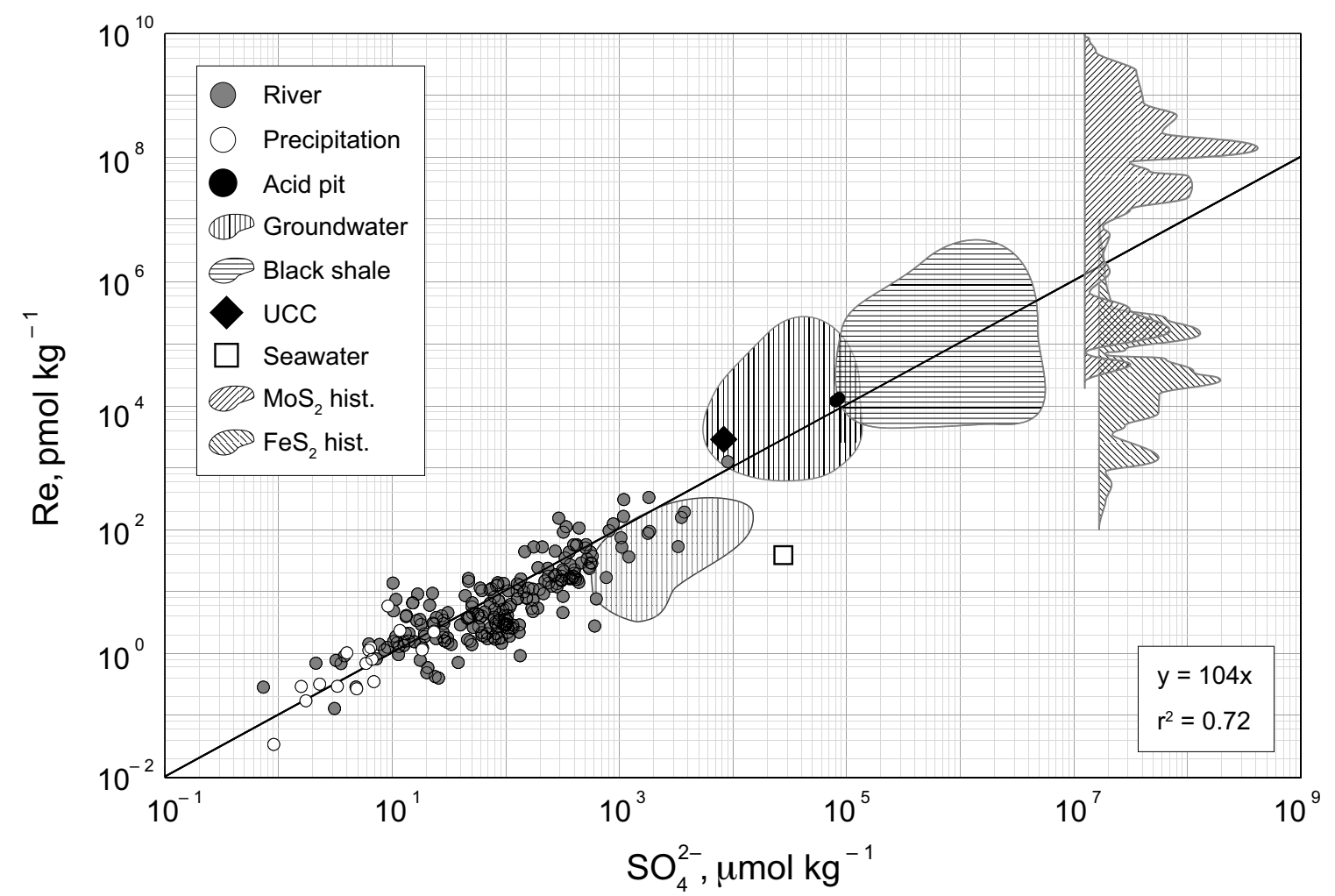

Figure 2-7: Rhenium and $\mathrm{SO}_{4}^{2-}$ in surface waters, crustal rocks, and sulfides.

Also plotted is a regression line fit to river data and forced through the origin.

$\log _{10}$-scaled histograms of Re concentrations in $\mathrm{MoS}_{2}(\mathrm{n}=320)$ and $\mathrm{FeS}_{2}+\mathrm{CuFeS}_{2}+\mathrm{AsFeS}_{2}$ $(\mathrm{n}=175)$ are also shown.

$\mathrm{UCC}=$ upper continental crust, Precipitation $=$ rain and snow collected in Falmouth, MA, USA between 2004/09/14 and 2005/09/29, Acid pit = samples of Berkeley Pit open pit mine waters, Butte, MN, USA.

Data for groundwaters, black shales, molybdenites and pyrites are taken from NoDDACK and Noddack (1931); Fleischer (1959, 1960); Morachevskil and Nechaeva (1960); Terada et Al. (1971); Ravizza and Turekian (1989); McCandless et Al. (1993); Hodge et Al. (1996); Freydier et Al. (1997); Brügmann et Al. (1998); Markey Et AL. (1998); Stein Et AL. (1998); Cohen Et AL. (1999); Mathur et AL. (1999, 2000); Peucker-Ehrenbrink and Hannigan (2000); Stein et Al. (2000); Kirk et Al. (2001); Selby and Creaser (2001); Arne et al. (2001); Stein et al. (2001); Creaser et al. (2002); Kirk et Al. (2002); Barra et AL. (2003); MaO et Al. (2003); Sun et al. (2003b); Hannah et Al. (2004); Berzina et Al. (2005); Mathur et Al. (2005); Morelli et Al. (2005); Zhang et Al. (2005); Brumsack (2006); Mao et al. (2006); Morelli Et Al. (2007); Selby et Al. (2007b); Leybourne and Cameron (2008); Liu et al. (2008). 
rich minerals, it is not surprising that $\mathrm{Re}$ and $\mathrm{SO}_{4}^{2-}$ exhibit a better correlation than Mo and $\mathrm{SO}_{4}^{2-}$.

\subsubsection{Estuarine behavior of Mo and Re}

The oxidized and dissolved $\mathrm{Mo}$ and Re species, $\mathrm{MoO}_{4}^{2-}$ and $\mathrm{ReO}_{4}^{-}$, are soluble and stable under oxic conditions (LEvinson, 1974). This stability is also seen in the ratios $\frac{[\text { metal }]_{\text {rivers }}}{[\text { metal }]_{\text {crust }}}$ and $\frac{[\text { metal }]_{S W}}{[\text { metal }]_{\text {crust }}}$ (after VISTELIUS AND SARMANOV, 1967). The value of $\frac{[\text { metal }]_{\text {rivers }}}{[\text { metal }]_{\text {crust }}}$ for Re is the highest of all metals while that of Mo is only exceeded by $\mathrm{Li}, \mathrm{B}, \mathrm{Na}, \mathrm{Mg}, \mathrm{K}, \mathrm{Ca}$, and Ag (calculated using data from this study and MASON AND MoORE, 1982; MCLEnNAN, 2001); values of $\frac{[\text { metal }]_{S W}}{[\text { metal }]_{\text {crust }}}$ for Mo and Re are only exceeded by those of $\mathrm{Li}, \mathrm{B}, \mathrm{Na}, \mathrm{Mg}, \mathrm{K}, \mathrm{Ca}$, and $\mathrm{Sr}$ (calculated using data from Mason And Moore, 1982; Taylor And Mclennan, 1985). On the basis of this high stability, as well as their conservative behavior in seawater (MoRRIS, 1975; Collier, 1985; Anbar et Al., 1992; Colodner et Al., 1993a), we might expect them to behave conservatively in the estuarine environment as well.

Previous studies of Mo in the Goldstream, Southampton Water, and Gironde estuaries indicate conservative mixing between freshwater and seawater end members (Head and Burton, 1970; Berrang And Grill, 1974; Audry et Al., 2007); however, a survey of the Chao Phraya estuary DALAI ET AL. (2005) showed a nonconservative Mo addition at higher $(>5 \%$ ) salinities due to mobilization of Mo from reduction of Mn-oxides in the underlying sediments. In the Amazon estuary, Re exhibited broadly conservative mixing, but a possible non-conservative addition at the low-salinity $(<5 \%$ ) end was also observed. It appears that the estuarine behavior of Mo and Re must be evaluated on a case-by-case basis.

Figure 2-8 describes the conservative behavior of Mo and Re in the Mississippi River estuary sampled on November 9, 10, and 11, 2002. Both elements exhibit highly linear mixing $\left(\mathrm{Mo}, \mathrm{r}^{2}=0.994 ; \mathrm{Re}, \mathrm{r}^{2}=0.996\right)$ between freshwater and seawater end members. Note that the respective Mississippi River end member (0\% salinity) Mo 
and Re concentrations of $27 \mathrm{nmol} \mathrm{kg}^{-1}$ and $90 \mathrm{pmol} \mathrm{kg}^{-1}$ are different from those obtained from a summation of the major tributary time-series data $\left(21 \mathrm{nmol} \mathrm{kg}^{-1}\right.$ and $57 \mathrm{pmol} \mathrm{kg}^{-1}$ respectively). This is not unexpected as the modeled yearly concentrations of Mo and Re are expected to vary from $15-30 \mathrm{nmol} \mathrm{kg}{ }^{-1}$ and $35-85 \mathrm{pmol} \mathrm{kg}^{-1}$, respectively (Section 2.5.1).

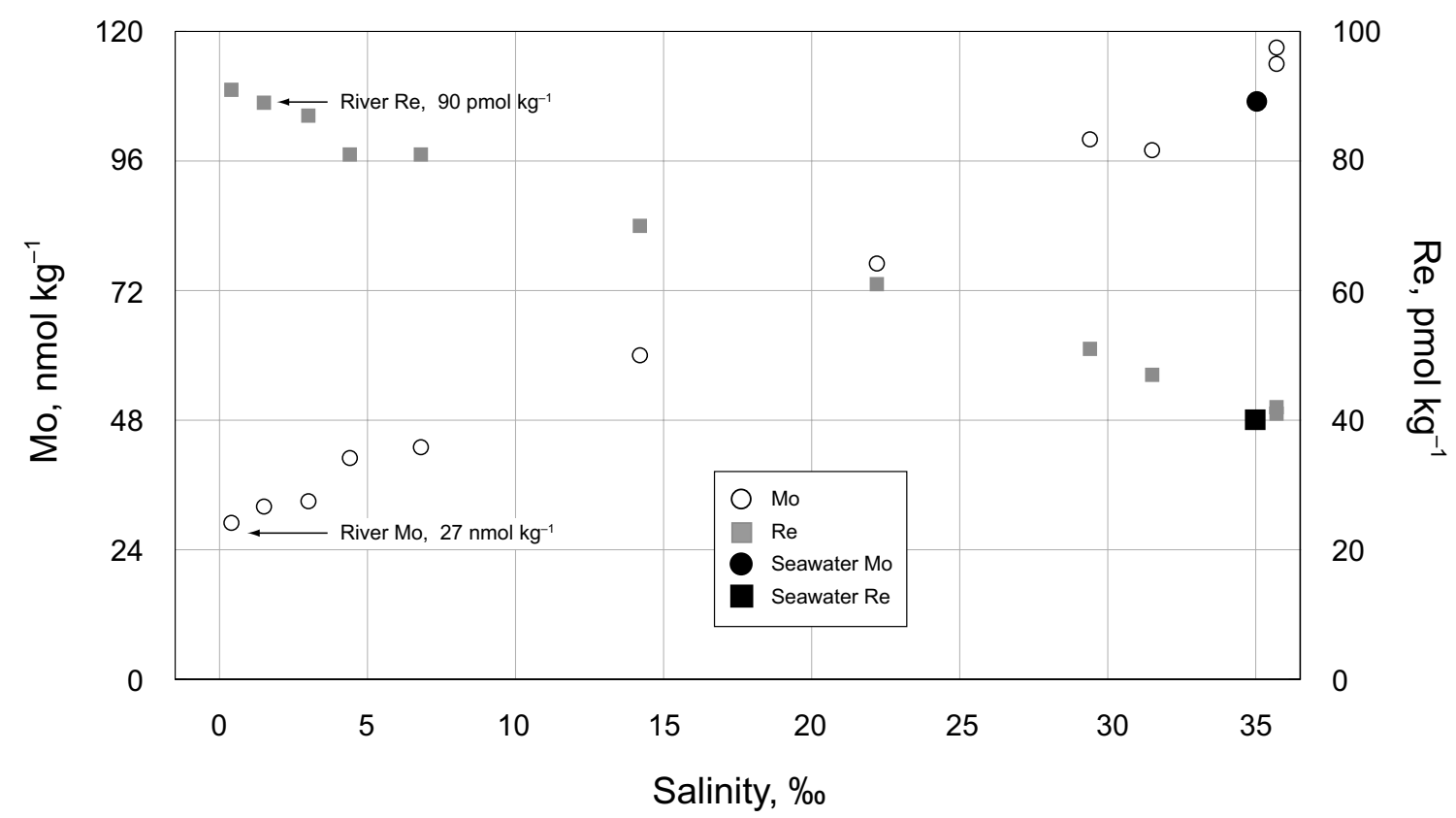

Figure 2-8: Molybdenum and Re concentrations vs. salinity, Mississippi River estuary, LA, USA.

Figure 2-9 shows the behavior of Mo and Re with salinity for four profiles of the Hudson River estuary taken on different sampling dates (see below). The Hudson river estuary begins at Battery Park at the southern end of Manhattan and extends 153 miles north to the Federal Dam at Troy, NY. While Mo consistently shows conservative behavior, Re exhibits a non-conservative addition at lower salinities. Though Profile 1 exhibits this feature across a wide salinity range (0-25\%), for Profile 3 it is strictly a freshwater $(<1 \%$ ) feature. Profile 4 may sample part of it as it does show an increase in Re concentration at the freshwater end.

When the metal concentration data are plotted with changing latitude for all available profiles (yr/m/d; Profile 1, 2004/07/07; Profile 2, 2006/06/06; Profile 3, 

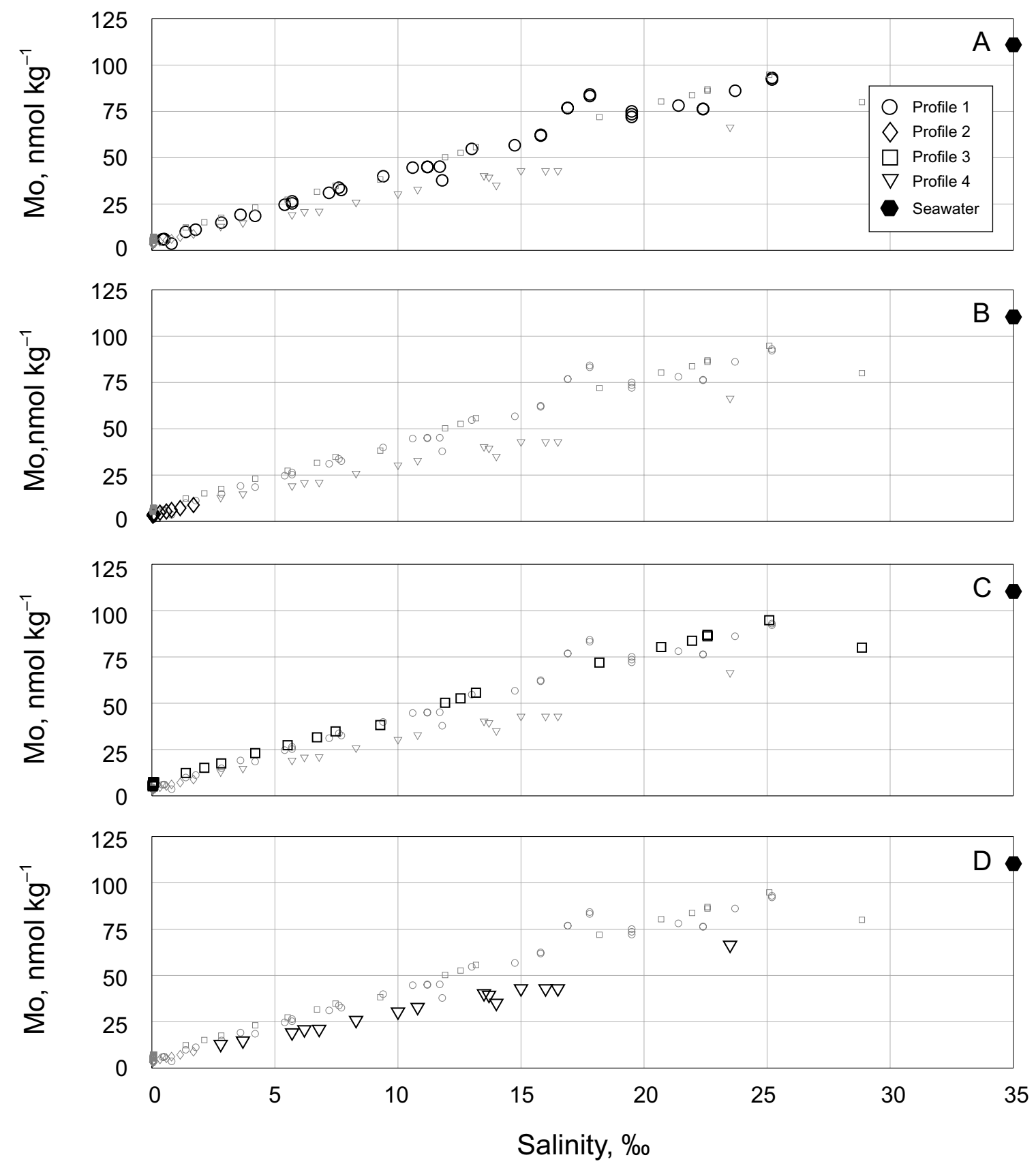

Figure 2-9: A-D: Molybdenum concentration vs. salinity, Hudson River estuary, NY, USA. Note that while all data are shown, each panel highlights data from a particular cruise.

A: Profile 1, taken 2006/07/07 (yr/m/d) aboard the $R / V$ Tioga (WHOI).

B: Profile 1, taken 2006/06/06 (yr/m/d) aboard the R. Ian Fletcher (Riverkeeper).

C: Profile 2, taken 2006/10/12 (yr/m/d) "Hudson River Snapshot Day," 2006 (organized by LDEO). Samples were taken from shore.

D: Profile 3, taken 2008/11/05 (yr/m/d) aboard the $R / V$ Tioga (WHOI). 

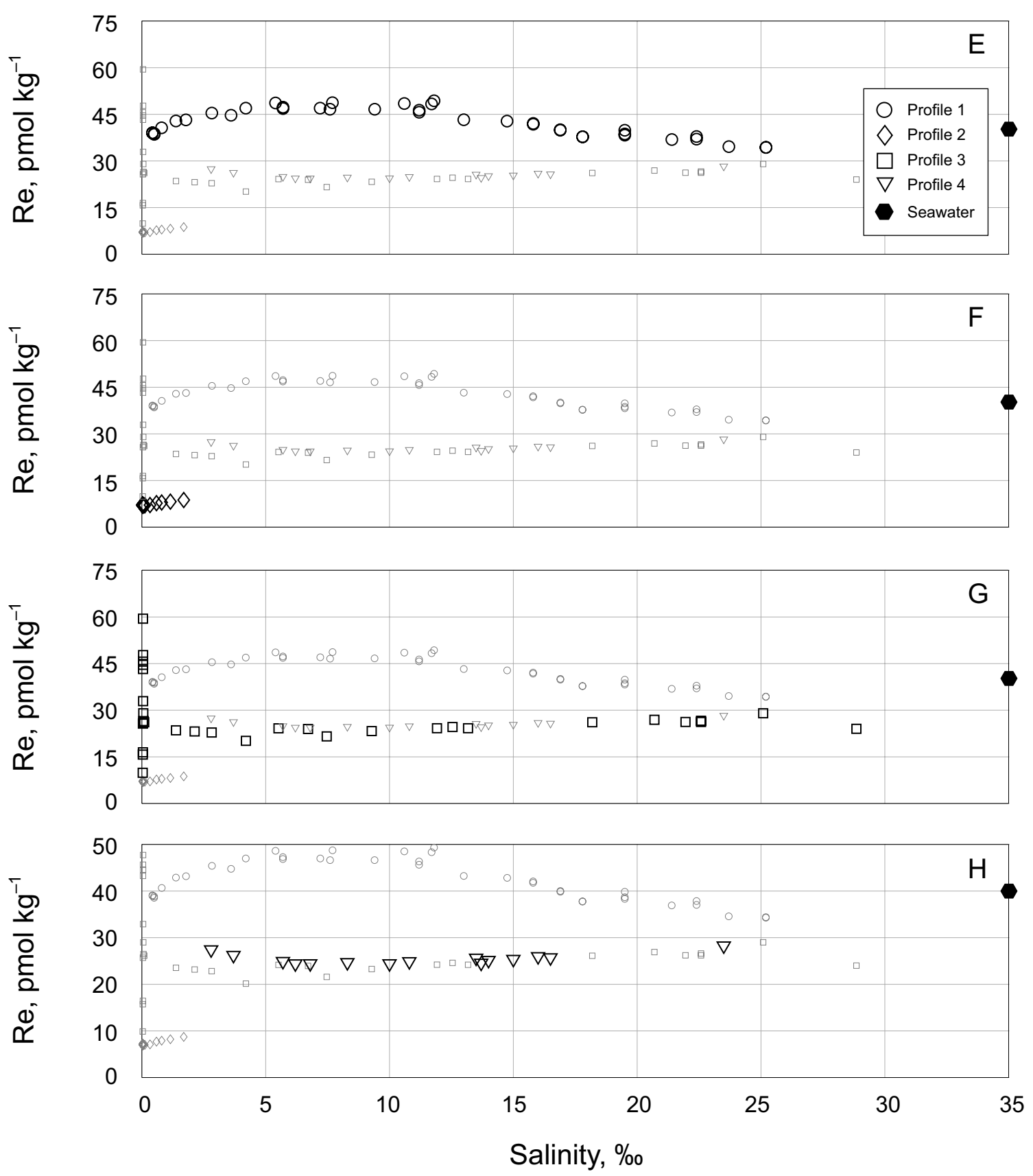

Figure 2-9: E-H: Rhenium concentration vs. salinity, Hudson River estuary, NY, USA. Note that while all data are shown, each panel highlights data from a particular cruise.

E: Profile 1, taken 2006/07/07 (yr/m/d) aboard the $R / V$ Tioga (WHOI).

F: Profile 1, taken 2006/06/06 (yr/m/d) aboard the R. Ian Fletcher (Riverkeeper).

G: Profile 2, taken 2006/10/12 (yr/m/d) "Hudson River Snapshot Day," 2006 (organized by LDEO). Samples were taken from shore.

H: Profile 3, taken 2008/11/05 (yr/m/d) aboard the $R / V$ Tioga (WHOI). 
2006/10/12; Profile 4, 2006/11/05; Profile 5, 2007/10/02; Profile 6, 2008/07/20; Fig. 2-10), we observe that the Re enrichment feature exhibits more consistent behavior on the basis of location rather than salinity. Profiles 1 and 3 capture essentially the entire enrichment feature, while Profiles 5 and 6 capture only part of it; Profile 2 does not appear to sample the enrichment feature at all (the increasing Re concentration in Profile 2 is accompanied by a salinity increase consistent with addition of seawater Re).

Examination of these various profiles allows us to discern several characteristics of the Re enrichment feature. (1) It is significant. Of the four profiles that unequivocally capture some part of the enrichment, the smallest of the Re concentration maxima is $\sim 50 \mathrm{nmol} \mathrm{kg}{ }^{-1}$; this is 7 times the Hudson River "background" concentration (based on Re concentrations of major tributaries, and the lowest observed Hudson Re concentrations of $\sim 7 \mathrm{pmol} \mathrm{kg}^{-1}$, Profile 2), and significantly higher than the seawater value of $40 \mathrm{pmol} \mathrm{Re} \mathrm{kg}^{-1}$. (2) It is not rare. Four of six (perhaps five of six) profiles capture some part of the enrichment feature; the two profiles in which the enrichment feature is not conclusively observed do not encompass the entire estuary, so we are not truly able to say that the feature is absent. (3) Though not rare, the feature can be transient; Profiles 1 and 3 show essentially the entire profile with decreasing Re concentration upstream and downstream of some maximum, indicating that the source of Re is not constant. (4) The Re enrichment feature is variable; rhenium

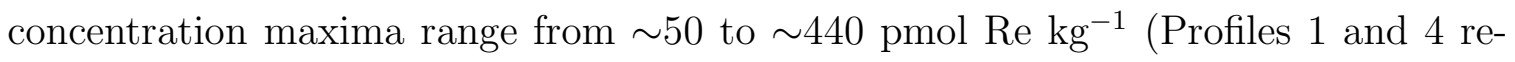
spectively). Locations of the Re enrichment maxima also vary; profiles showing the entire feature show Re maxima separated by two degrees of latitude. (5) The source of the enrichment feature appears to be located at or upstream of the Federal Dam in the city of Troy, NY. Though Profiles 4 and 5 fail to capture the entire profile, they both show their Re concentration maxima at Troy, NY. In addition, these profiles show Re concentrations that decrease with increasing distance from Troy in both the upstream and downstream directions. Profiles 4 and 5 also show the highest Re 

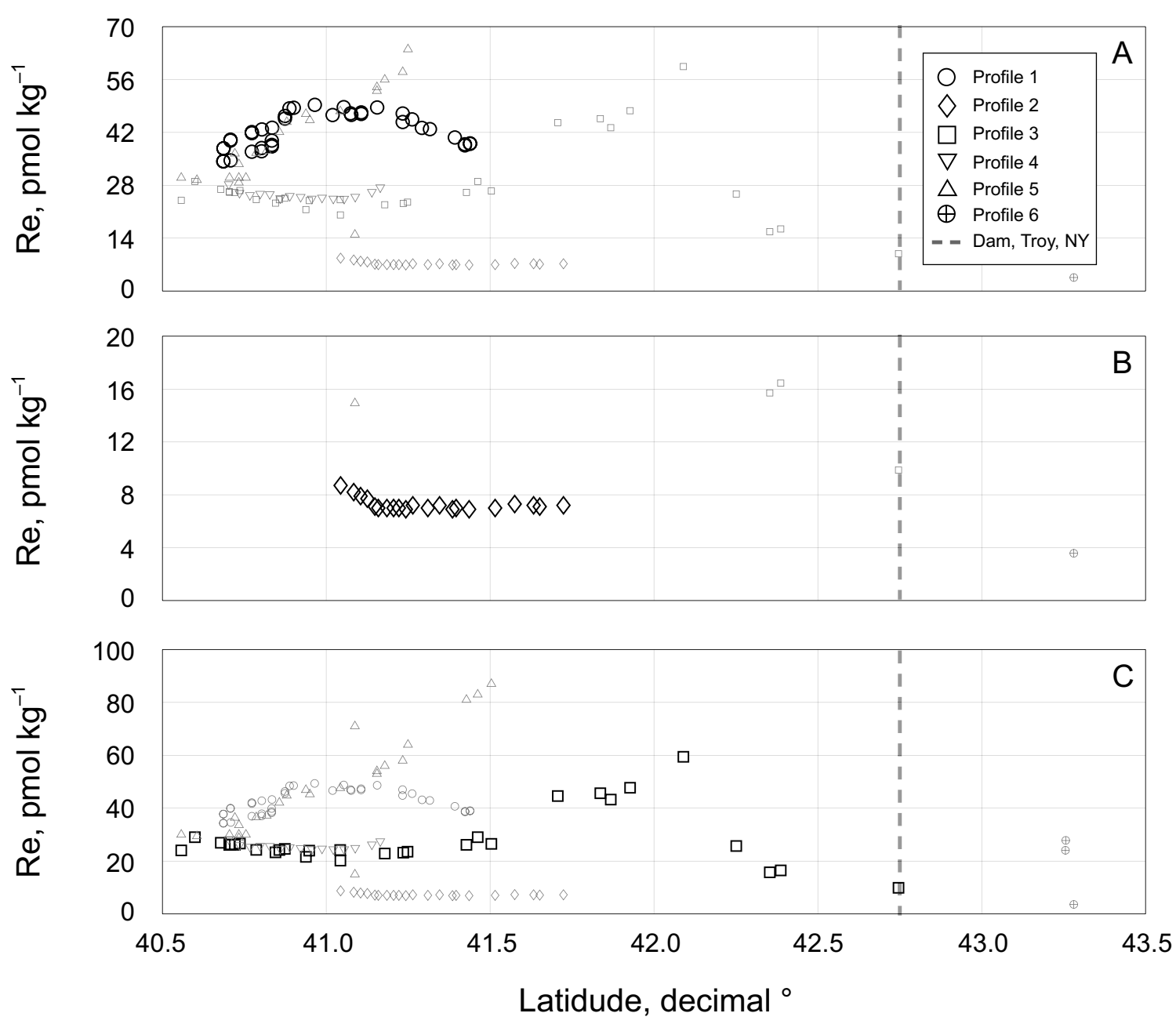

Figure 2-10: A-C: Rhenium concentration vs latitude, Hudson River, NY, USA. Note that while all data are shown, each panel highlights data from a particular cruise.

A: Profile 1, taken 2004/07/07 (yr/m/d) aboard the $R / V$ Tioga (WHOI).

B: Profile 2, taken 2006/06/06 (yr/m/d) aboard the R. Ian Fletcher (Riverkeeper).

C: Profile 3, taken 2006/10/12 (yr/m/d) "Hudson River Snapshot Day," 2006 (organized by LDEO). Samples were taken from shore. 

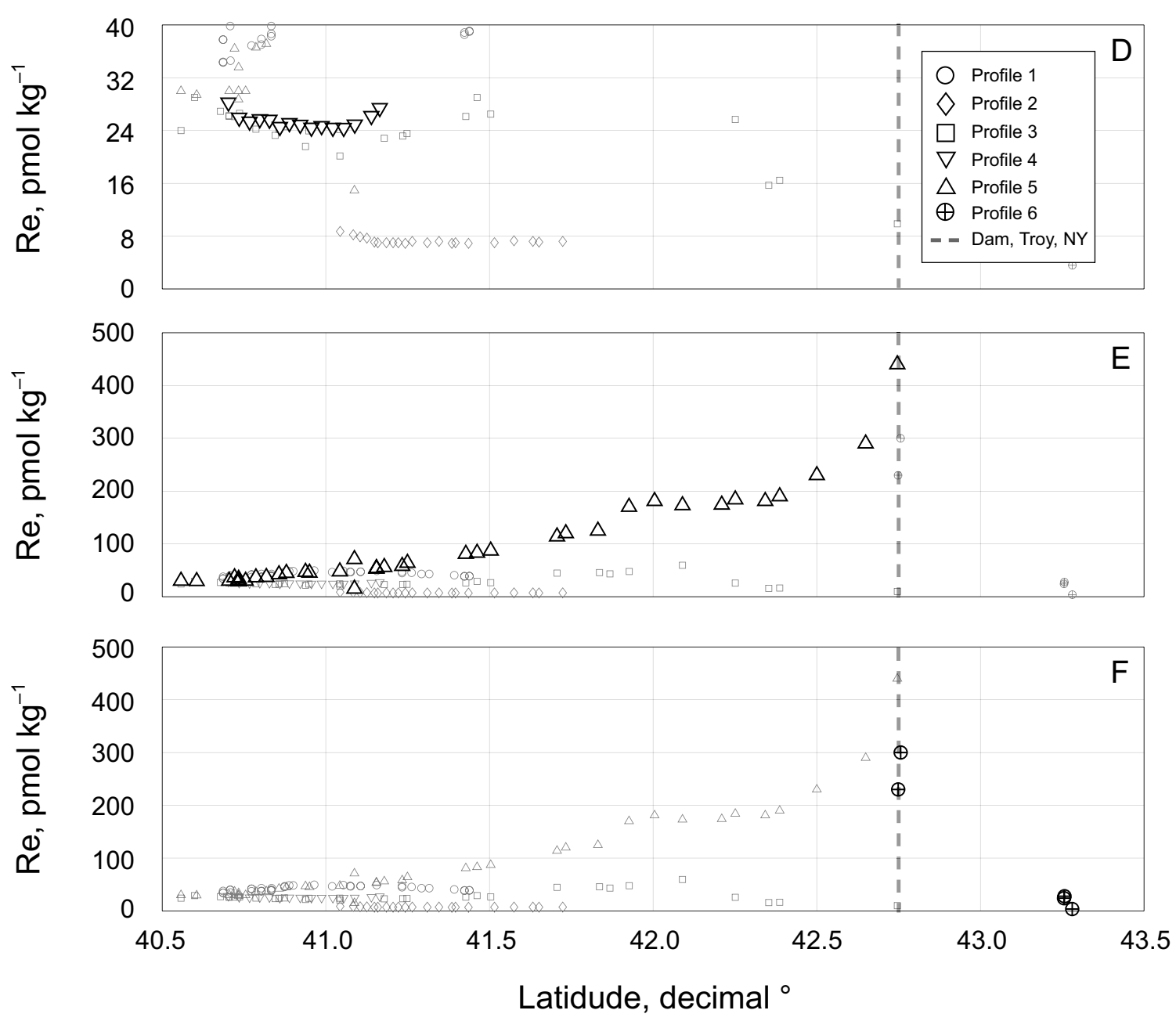

Figure 2-10: D-F: Rhenium concentration with latitude, Hudson River, NY, USA. Note that while all data are shown, each panel highlights data from a particular cruise.

D: Profile 1, taken 2006/11/05 (yr/m/d) aboard the $R / V$ Tioga (WHOI).

E: Profile 2, taken 2007/10/02 (yr/m/d) "Hudson River Snapshot Day," 2007 (organized by LDEO). Samples were taken from shore.

F: Profile 3, taken 2008/07/20 (yr/m/d) by the author (Miller). Samples were taken from shore. 
concentrations observed in the Hudson River (300 and $440 \mathrm{pmolkg}^{-1}$ ). (6) The enrichment feature is not related to freshwater-saltwater mixing. The presence of the feature upstream of Troy Federal Dam is significant as the dam marks the northern end of the Hudson River estuary (DE VRIES AND WEISS, 2001); saline waters do not travel past the dam.

The various profiles indicate that significant amounts of Re are added to the Hudson River above the Federal Dam at Troy, NY, on an intermittent basis (note the concentration of $10 \mathrm{pmol} \mathrm{Re} \mathrm{kg}^{-1}$ at the dam, Snapshot Day 2006, Fig. 2-10 C). Though concentrations for the Re-enrichment feature appear to reach their maxima at Troy, Profile 6 (Fig. 2-10 F) shows that Re concentrations can increase significantly above the background Hudson River level $\left(\sim 7 \mathrm{pmol} \mathrm{Re} \mathrm{kg}^{-1}\right)$ at considerable distances upstream of the Federal Dam; it also shows that the source of the enrichment may not be a point-source. The precise location(s) and nature of the Re source remain unclear.

The Mohawk river, a major tributary that joins the Hudson main stem at Troy, has Re concentrations (7.8 pmol $\mathrm{Re} \mathrm{kg}^{-1}$, Table 2.3) insufficient to account for the enrichment feature; likewise, an examination of other Hudson tributaries show even lower Re concentrations (Table 2.3). COLODNER ET AL. (1993a) observed a similar, though considerably smaller, low salinity Re-enrichment enrichment feature for the Amazon River estuary and suggested it may have been the result of Re desorption from suspended sediments or the release of Re sequestered in reducing estuarine sediments. Saline or brackish waters are not prerequisite for the presence of the Hudson Re enrichment feature (Profiles C, E, F), and its occurrence in waters upstream of the Federal Dam indicate that it is not the result of an estuarine mixing process. Release of Re from the re-suspension and oxidation of reducing sediments should result in a coincident release of Mo and S, but no Mo enrichment feature has been observed and the data of Profile 3 show a $30 \mathrm{pmol} \mathrm{kg}^{-1}$ addition of Re without any observed increase increase in $\mathrm{SO}_{4}^{2-}$ (Table 2.5). Though weathering of Re-rich lithologies such as 
black shales are known to result in high concentrations of dissolved Re (COLODNER et Al., 1993a; Dalai et Al., 2002; Pierson-Wickmann et Al., 2003) and while there are significant black shale exposures in the Hudson drainage basin (including in the city of Troy), weathering of these lithologies typically results in consistently high concentrations of dissolved Re and $\mathrm{SO}_{4}^{2-}$ (COLODNER ET AL., 1993a). As has been mentioned, there is no coincident $\mathrm{SO}_{4}^{2-}$ enrichment, and the Re enrichment feature is not consistently observed.

The intermittent feature seen in the Hudson River represents a 7-60 fold Re enrichment over the "background" river concentration. Given the population density and industrial history of the region, it is possible that the sporadic Re enrichment is anthropogenic. Previous studies document anthropogenic contributions of heavy metals to the sediments of the Hudson (Feng et AL., 1998; Benoit et AL., 1999) as well as other rivers (e.g. LI ET AL., 2000; WoItKe ET AL., 2003). At the moment, not enough is known about the frequency, severity, and longevity of these enrichments to determine the degree to which they enhance the background Hudson Re flux.

\subsubsection{Mo and Re in high temperature hydrothermal fluids from the Manus Basin}

Since the discovery of seafloor hydrothermal vents (CORLISS ET AL., 1979), characterization of hydrothermal fluids has been important to constrain chemical fluxes to and from seawater (EDmOND ET AL., 1979). These fluxes can be significant as some metals are present in hydrothermal fluids at concentrations orders of magnitude greater than in seawater (e.g. FouQuet ET AL., 1991).

Analyses of high-temperature hydrothermal fluids found Mo concentrations significantly lower than seawater (Trefry et AL., 1994; Metz AND Trefry, 2000), while lower-temperature $\left(\sim 25^{\circ} \mathrm{C}\right)$ ridge-flank hydrothermal systems show Mo concentrations several fold higher than seawater (WhEAT ET AL., 2002). Using these concentrations and hydrothermal water flux estimates (ELDERfIELD AND SCHULtz, 
1996), the Mo hydrothermal flux to seawater is estimated as $14 \%$ of the previously estimated riverine flux (WheAt ET AL., 2002).

Prior to this study, there were no published values of the Re concentration of hydrothermal fluids. Lab-based modeling studies of Re in hydrothermal systems (XIONG AND Wood, 1999, 2001, 2002) indicate that while Re concentrations could be high in high-temperature brines due to $\mathrm{Cl}^{-}$-complexation, such waters in equilibrium with sulphide minerals could have very low Re concentrations because of the precipitation of sulphide minerals. Supporting this, high concentrations of Re $\left(10^{0}-10^{2} \mathrm{ngg}^{-1}\right)$ have been observed in hydrothermal sulfides (KOIDE ET AL., 1986; ROY-BARMAN ANd Allègre, 1994; Ravizza et AL., 1996).

Data for four hydrothermal fluid samples and one bottom seawater sample from the Roman Ruins vent site, Papua New Guinea-Australia-Canada-Manus (PACMANUS) hydrothermal field, Manus Basin, are presented in Table 2.6. Fluids were sampled in August and September, 2006 during $R / V$ Melville cruise MGLN06MV using the ROV JasonII. Samples were collected in $755 \mathrm{~mL}$ Ti-syringe samplers and extracted immediately after completion of dive operations. Rapidly-precipitating sulphides ("dregs," see Table 2.6) were collected on a $0.22 \mu \mathrm{m}$ filter using Milli-Q water; slowly-precipitating sulfides ("bottle filtrate," see Table 2.6) were isolated on-shore by $0.22 \mu \mathrm{m}$ filtration. Particulates were removed from filters by rinsing with concentrated Optima Grade $\mathrm{HNO}_{3}$. More details are available in (CRADDOCK, 2008).

The sampling of hydrothermal fluids introduces artifacts not only due to the entrainment of ambient seawater but also from sulfide mineral precipitation caused by temperature and pressure changes experienced by the sample between the time it is collected and the time it is analyzed. Because Mo and Re may precipitate as sulfides, reincorporation of the metal proportions present in these phases with the metal proportions of the dissolved phase is crucial in determining metal concentrations in the original fluid. For this study, mineral precipitates are isolated and redissolved into a solution of known mass. The sampled fluid mass (assumed to be $750 \mathrm{~g}$ ) as well as the 
dregs, and bottle filtrate solution masses are presented in Table 2.6, and are used to reconstruct the original hydrothermal fluid samples. Concentrations of $\mathrm{Mg}$ for these samples are also included (Table 2.6) to allow subtraction of the entrained seawater metal fraction.

Table 2.6 indicates that large proportions of the Mo and Re in these hydrothermal fluids ( $\sim 81 \%$ and $\sim 61 \%$, respectively) are present in the sulphide fractions (dregs+bottle filtrate). The predominance of these metals in the sulphide mineral fraction has already been observed for Mo (TREFRY ET AL., 1994) and is predicted for Re (XIONG AND WoOd, 2001, 2002). Also, the reconstructed metal concentrations for the fluids as they were sampled (i.e., not Mg-corrected) are all lower than the accepted (COLLIER, 1985; ANBAR ET AL., 1992) or locally observed seawater values, indicating that high-temperature hydrothermal circulation acts as a sink for these metals. The Mo/Mg and $\mathrm{Re} / \mathrm{Mg}$ ratios of these reconstituted fluids are all less than the local seawater values of $2.2 \mathrm{nmol} / \mu \mathrm{mol}(\mathrm{Mo} / \mathrm{Mg})$ and $0.61 \mathrm{pmol} / \mu \mathrm{mol}(\mathrm{Re} / \mathrm{Mg})$, meaning that these fluids are depleted in Mo and Re. This deficit cannot be the result of reduction of Mo and Re into sulfides that are then precipitated because it is the reconstituted fluid values that show the deficit. Assuming that the end-member fluid compositions are Mg-free (SEyfried And Mottl, 1982; SEyfried, 1987) even end-member fluids that are metal-free should display Mo and Re concentrations at levels consistent with the $\mathrm{Mg}$ concentration of the sampled fluid. The unexpectedly low $\mathrm{Mo} / \mathrm{Mg}$ and $\mathrm{Re} / \mathrm{Mg}$ ratios suggest that end-member compositions of these sampled fluids contain $\mathrm{Mg}$, and that Mo and Re are more rapidly removed during hydrothermal seafloor alteration than Mg. This may serve to explain the large negative Mg-corrected Mo concentration value observed for sample J2 208 M2. Though three of four hydrothermal fluid samples also exhibit negative Re concentrations, they are within uncertainty of zero in light of the various assumptions made during the reconstruction of the fluid sample concentrations, for example, the assumption that the initial fluid mass was $750 \mathrm{~g}$. The J2 $208 \mathrm{M} 2$ Mo concentration of $-27 \mathrm{nmol} \mathrm{kg}^{-1}$ is far 
more likely to be significant. It should also be noted that negative Mo concentrations have been observed in other studies (Trefry ET AL., 1994; Metz And Trefry, 2000). Essentially, the assumption of zero-Mg in the end-member fluid resulted in a negative Mo concentration for J2 208 M2 because the Mo/Mg ratio of the ambient bottom water was used in the $\mathrm{Mg}$ correction. Should these end-member fluids all contain $\mathrm{Mg}$, the Mo and Re concentrations reported in Table 2.6 are all minimum values.

The Mg-corrected metal concentrations indicate that Re is essentially absent from these fluids, and that Mo is present at levels consistent with those reported from other high-temperature vent sites (Trefry ET AL., 1994; Metz And Trefry, 2000). Assuming that end-member fluids are Mg-free and that Mo and Re concentrations listed in Table 2.6 are representative of high-temperature hydrothermal fluids (Mo $\sim 22 \mathrm{nmolkg} \mathrm{kg}^{-1}$, Re $\sim 1.4 \mathrm{pmol} \mathrm{kg}^{-1}$; all negative values assumed to be 0 ), a high-

temperature hydrothermal water flux of $3 \times 10^{13} \mathrm{~kg} \mathrm{yr}^{-1}$ (ELDERFIELD AND SCHULTz,

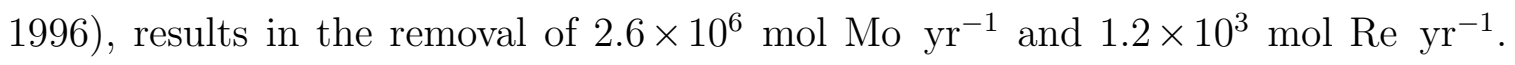
These fluxes correspond to the approximately $0.4 \%$ and $0.1 \%$ of the respective modern Mo and pre-anthropogenic Re river fluxes to seawater presented in Section 2.5.2. High-temperature hydrothermal alteration is obviously not a significant sink for Mo and Re in seawater.

\subsubsection{Response times and modeling of Mo and Re inventories in seawater}

The major sources of Mo to seawater are river water, $3.09 \times 10^{8} \mathrm{molyr}^{-1}$, (Section 2.5.2) and low temperature hydrothermal fluids, $2.62 \times 10^{7} \mathrm{molyr}^{-1}$ (assuming a flux $13 \%$ of the previous riverine flux estimate, METz And TrEFry, 2000, and a global river water discharge of $3.86 \times 10^{16} \mathrm{~kg} \mathrm{yr}^{-1}$, FEKETE ET AL., 2002). The major source of Re to seawater is the dissolved river flux, $4.32 \times 10^{5} \mathrm{molyr}^{-1}$ (preanthropogenic, Section 2.5.3). The low-temperature hydrothermal Re flux cannot be 
evaluated due to a lack of data; we assume these fluids to be negligible sources of seawater Re. Sinks of oceanic Mo and Re are more difficult to identify and quantify. For example, estimates of Mo removal in sediments overlain by anoxic bottom waters done using mass-flux calculations span an order of magnitude $-8.6 \times 10^{7}$, $2.0 \times 10^{7}$, and $9.5 \times 10^{6} \mathrm{molyr}^{-1}$ (respectively EVANS ET AL., 1978; BERTINE AND Turekian, 1973; Algeo, 2004). In addition, Evans et Al. (1978) and Bertine AND TuREkiAn (1973) calculate the anoxic sink flux by difference in an attempt to balance the previous riverine source flux; the new flux calculated in Section 2.5.2 will, therefore, significantly increase these estimates. SiEBERT ET AL. (2003) employ Mo isotopes to quantify the proportions of anoxic and oxic sink fluxes $(70 \%$ and $30 \%$, respectively). As other potential sinks are less understood (e.g. MoRFORD AND Emerson, 1999; Poulson et AL., 2006), seawater response times of Mo and Re are calculated and compared using seawater inventories and metal fluxes to seawater.

The response time $(\tau)$ of a system characterizes its re-adjustment to equilibrium after a perturbation; for a reservoir with first order sink fluxes, it is also called the turnover time and is expressed as the ratio of the magnitude of the reservoir to the magnitude of the flux out $\left(M / f_{\text {out }}\right.$; RoDHE, 1992). We consider the reservoir to be the seawater metal inventories, and fluxes out are assumed to equal the fluxes in. Using seawater concentrations of $104 \mathrm{nmol} \mathrm{kg}^{-1}$ (Mo, MORRIS, 1975; COLLIER, 1985) and 40 pmol kg-1 (Re, Anbar ET AL., 1992; Colodner ET AL., 1993a, 1995) along with an oceanic volume of $1.35 \times 10^{21} \mathrm{~L}(\mathrm{MENARD}$ AND SMITH, 1966), an average seawater density of $1.028 \mathrm{kgL}^{-1}$ (after Montgomery, 1958 and Millero And Poisson, 1981), and a global river water discharge of $3.86 \times 10^{16} \mathrm{~kg} \mathrm{yr}^{-1}$, we obtain Mo and Re response times of $7.9 \times 10^{5} \mathrm{yr}\left(\tau_{\mathrm{Mo}}\right)$, and $6.5 \times 10^{5} \mathrm{yr}\left(\tau_{\mathrm{Re}}\right)$ for previous estimates of the world river averages (Bertine And Turekian, 1973; Colodner ET AL., $1993 \mathrm{a})$, and times of $4.4 \times 10^{5} \mathrm{yr}\left(\tau_{\mathrm{Mo}}\right)$, and $1.3 \times 10^{5} \mathrm{yr}\left(\tau_{\mathrm{Re}}\right.$, pre-anthropogenic flux) for the new world river average estimates obtained in this study (Sections 2.5.2 and 2.5.3). The $\tau_{\mathrm{Re}}$ corresponding to the modern Re flux is $8.2 \times 10^{4} \mathrm{yr}$. 
This study has revised the Mo and Re seawater response times downwards, meaning that the seawater inventories of both metals are more sensitive to changing source or sink fluxes than was previously thought. Previous estimates of $\tau_{\text {Mo }}$ and $\tau_{\text {Re }}$ were not only longer than our revised estimates, they were also quite similar to one another $\left(\tau_{\operatorname{Re}}\right.$ was $82 \%$ of $\left.\tau_{\mathrm{Mo}}\right)$; our new data indicate that pre-anthropogenic $\tau_{\mathrm{Re}}$ is $30 \%$ of $\tau_{\mathrm{Mo}}$, allowing these two metals to be used for tracking processes acting on different timescales.

Other studies have noted the high concentrations of reductively-enriched metals in modern anoxic sediments and have hypothesized that the expansion of depositional environments of this type could conceivably decrease the oceanic inventory of these metals (Emerson And Huested, 1991; Colodner et Al., 1993a; Algeo, 2004). Here we present a model to compare the effects of differing anoxic sink fluxes on the seawater inventories of Mo and Re for new and previous assessments of $\tau_{\mathrm{Mo}}$ and $\tau_{\mathrm{Re}}$.

The mathematical details of the model are outlined in Section 2.A. It is assumed that the modern ocean is at steady-state with respect to Mo and Re, that the source fluxes are constant, that the sink fluxes are first order, and that the flux constant for the anoxic flux varies varies with time, but is constant over 100,000 yr intervals. Because the modern riverine Re flux shows significant anthropogenic enhancement, it is unlikely that modern seawater is at steady-state for Re; as a result, we use the preanthropogenic Re flux. It is also assumed that the oxic and anoxic flux proportions are respectively $70 \%$ and $30 \%$ of the source flux (SIEBERT ET AL., 2003) for both Mo and Re. This may be an oversimplification because anoxic conditions are required for Mo enrichment while Re can be enriched under suboxic conditions (CRUSIUS ET AL., 1996; Morford AND EmERson, 1999).

Model results are presented in Figure 2-11. Figure 2-11 A illustrates the model response of Mo and Re seawater inventories assuming previous estimates of $\tau_{\text {Mo }}$ and $\tau_{\text {Re. }}$ Because the response times were relatively long, Mo and Re seawater inventories show modest $(<5 \%)$ decreases over the 100,000 yr time intervals with an enhanced 
anoxic sink flux. Also, because of the similarity in previous estimates of the response times, Mo and Re inventories follow very similar patterns.

Figure 2-11 C illustrates the model response assuming $\tau_{\mathrm{Mo}}$ and pre-anthropogenic $\tau_{\mathrm{Re}}$ values determined in this study. Seawater inventories of both Mo and especially Re both decrease to larger degrees over the periods of enhanced anoxic sink fluxes due to the shorter response times. The seawater Mo inventory decreases by $\sim 5 \%$ while that of Re decreases by $>10 \%$ over the same 100,000 yr interval. Clearly, Re is much more sensitive to changing sink (or source) fluxes than Mo; studies attempting to identify changing sink fluxes in the geologic past using trace metal concentrations or ratios should evaluate Re as well as Mo.

More importantly, these model results also apply to changing isotopic compositions of seawater Mo, and perhaps Re as well. For example, a recent study (PEARCE ET AL., 2008) observed $2 \%$ shifts in $\delta^{98 / 95}$ Mo corresponding to Jurassic ocean anoxic events (OAEs) across time-spans of $<50,000$ yr. Pearce et AL. (2008) use the model of LING ET AL. (2005) to estimate that the decreases in isotopic ratio correspond to a roughly 10-fold expansions of the anoxic sink. However, LiNG ET AL. (2005) assume that discrete changes in $\delta^{98 / 95}$ Mo correspond to explicit increase in the size of the sink flux; there is no associated time-function to account for the degree of re-equilibration over the interval of interest. The changing isotopic composition of the seawater is seen in the decreasing isotopic values across the anoxic events. Though the isotope systematics of Re in the modern ocean has not been evaluated, Re isotopic composition can vary systematically across a redox gradient (MilLER ET AL., 2008, Chapter 4). Should oxic, anoxic, and suboxic sinks fractionate Re isotopes differently (as is seen for Mo), we can expect the $\delta^{187}$ Re of seawater will re-equilibrate much more quickly than $\delta^{98 / 95} \mathrm{Mo}$. 

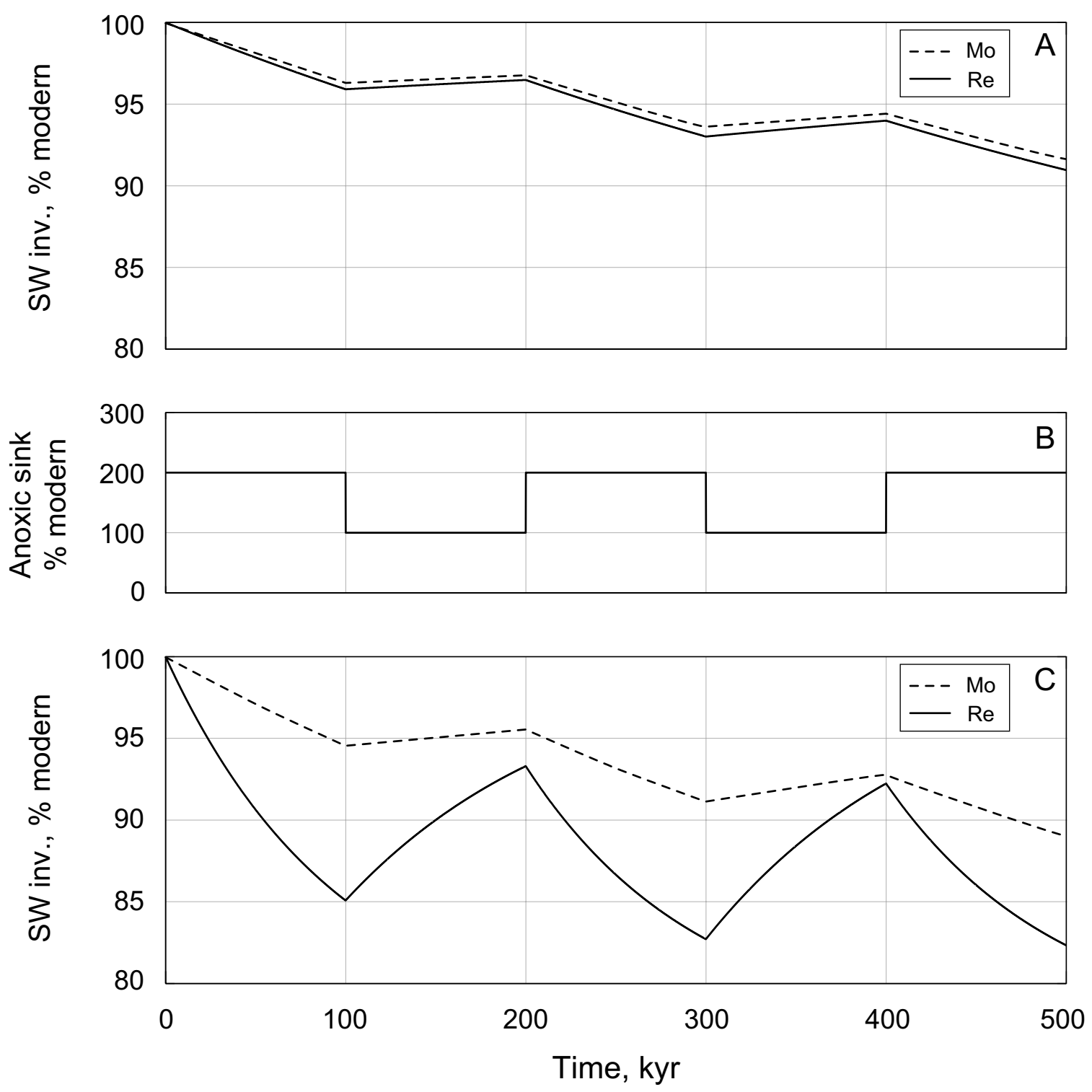

Figure 2-11: Results of modeling Mo and Re seawater inventories.

A: Seawater inventories of Mo and Re assuming previous world river average concentrations and seawater response times (Bertine And Turekian, 1973; Colodner et Al., 1993a).

B: Changing anoxic sink flux relative to modern proportions determined for Mo (SIEBERT ET AL., 2003).

C: Seawater inventory results for Mo and Re assuming new world river average Mo concentration, pre-anthropogenic Re river concentration, and associated seawater response times (this study). 


\subsection{Conclusions}

This study used a set of 38 global rivers encompassing $37 \%$ of total water discharge and $25 \%$ of exorheic continental drainage area to calculate the world river average

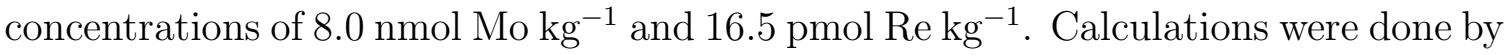
summing flux-weighted metal concentrations for Graham drainage regions (GRAHAM ET AL., 1999). This type of calculation produces world river average cation concentrations consistent with published values calculated using other methods (LIVINGSTONE, 1963; Meybeck, 1979; Meybeck And RaGU, 1995); calculated anion river averages (especially $\mathrm{Cl}^{-}$) for our samples were significantly higher than the published values. Our re-calculated Mo and Re values are significantly higher than the previous estimates of $4.5 \mathrm{nmol} \mathrm{kg}^{-1}$ and $2.1 \mathrm{pmol} \mathrm{kg}^{-1}$ respectively (BERTINE AND TUREKIAN, 1973; Colodner et AL., 1993a).

Some samples indicate an anthropogenic component to these new river averages, particularly for Re. Corresponding Mo enrichments for these samples are nonexistent or only moderate, indicating that Re might be a sensitive indicator of anthropogenic metal contamination. Using the observed correlation between $\mathrm{Re}$ and $\mathrm{SO}_{4}^{2-}$ we estimate that $32 \%$ of riverine Re is anthropogenically sourced. The pre-industrial average Re concentrations for rivers is $11.2 \mathrm{pmol} \mathrm{kg}^{-1}$. The equivalent natural Re riverine flux is $4.32 \times 10^{5} \mathrm{molyr}^{-1}$.

On the basis of a consistent and wide-ranging relationship between $\mathrm{Re}$ and $\mathrm{SO}_{4}^{2-}$ (Colodner ET AL., 1993a; DAlai ET AL., 2002), the source of Re to rivers is believed to be sulphide mineral and black shale weathering. Though these same sources are also invoked as the source of Mo to rivers, no significant relationship between Mo and $\mathrm{SO}_{4}^{2-}$ is observed.

Though both Mo and Re behave conservatively for the Mississippi River estuary, only Mo is conservative in the Hudson estuary. Rhenium exhibits a non-conservative addition centered around or upstream of the Federal Dam at Troy, NY. This Re enrichment is variable but significant (concentration maxima ranging from 50-440 


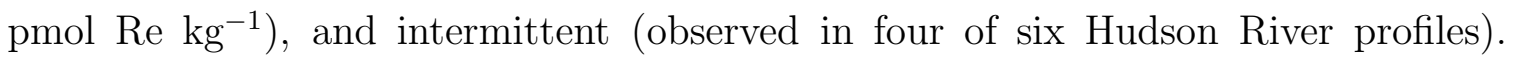
Because the feature is also observed in the fresh water upstream of the Federal Dam, it is not the result of estuarine mixing. The Re enrichment is not caused by the release of sediment-hosted Re via resuspension and oxidation as there are no equivalent Mo or $\mathrm{SO}_{4}^{2-}$ enrichment features. Given the high population density and industrial history of the Hudson River, it is possible that the feature is anthropogenically-sourced.

This study also presents the first data on Re in hydrothermal fluids. High temperature hydrothermal fluids from the Manus Basin show Mo and Re concentrations much lower than those of ambient seawater. In particular, Re was essentially absent in calculated end-member hydrothermal fluids. The data indicate that Mo and Re are removed from seawater more effectively than Mg during high-temperature hydrothermal alteration. High temperature hydrothermal fluids represent a negligible sink in comparison to the river source of these metals.

Using the new world river Mo and pre-anthropogenic river Re concentration averages, we have recalculated modern respective Mo and Re response times $\left(\tau_{\mathrm{Mo}}\right.$, preindustrial $\left.\tau_{\mathrm{Re}}\right)$ as $4.4 \times 10^{5} \mathrm{yr}$ and $1.28 \times 10^{5} \mathrm{yr}$, respectively. These response times indicate that Mo and especially Re will re-equilibrate more quickly to changing metal sources and sinks than was previously thought (COLODNER ET AL., 1993a; MORFORD AND EMERSON, 1999).

\subsection{Acknowledgements}

We would like to acknowledge financial support from NSF-EAR grant 0519387 and from the WHOI Academic Programs Office. We would like to thank Tracy Atwood, current and former members of the WHOI Plasma Mass Spectrometry Facility: Lary Ball, Scot Birdwhistell, Jerzy Blusztajn, and Dave Schneider, as well as the members of the Seewald Lab (WHOI): Eoghan Reeves, Jeff Seewald, and Sean Sylva for their analytical assistance. This work would not have been possible without the generous 
assistance of many friends and collaborators who provided us with individual water samples from all over the world: Bridget Bergquist, Peter Clift, Paul Craddock, Ted Duaime, Ricardo Figueroa, Jérôme Gaillardet, Valier Galy, Rocky Geyer, Liviu Giosan, Jaime Hills, Max Holmes, Matt Jackson, John Lipscomb, Tom Lough, Candace Martin, Daniel Montluçon, Petra Ehrenbrink, Greg Ravizza, Laura Robinson, Olivier Rouxel, Andrew Schroth, Michael Schubert, Malcolm Scully, Jeff Seewald, Ken Sims, Anna Skulasdöttir, Meg Tivey, Rhian Waller, Nadine Zimmer, Yan Zheng. In particular, we would also like to thank Rocky Geyer and Malcolm Scully (both WHOI) for for the collection of Hudson estuarine profiles. We would also like to thank John Lipscomb, Captain of the Riverkeeper patrol boat R. Ian Fletcher, for his assitance in sampling a Hudson estuary profile. Many thanks to Margie Turrin (LDEO) who coordinated the Hudson River "Snapshot days." Thanks also to all the volunteers, teachers, and students participating in Snapshot Day 2006 and 2007 who collected the samples. Samples were also provided to us by the NSF-supported PARTNERS and ARCTIC-GRO programs. We also thank Ed Sholkovitz for his suggestion of syringe-filtration for water processing, which made the above collaborations possible. 


\section{A Modeling seawater inventories of Mo and Re}

We evaluate the implications of our new Mo and Re world river averages by considering a model that makes four assumptions: (1) the modern ocean is at steady-state with respect to Mo and Re, (2) the riverine fluxes of Mo and Re remain constant with time, (3) oxic and anoxic fluxes of these metals out of seawater are first order, and (4) the magnitude of the anoxic flux has varied with time (SIEBERT ET AL., 2003; Arnold et Al., 2004; Pearce et Al., 2008).

Inputs of the model are the modern inventories of Mo and Re in seawater, the fluxes of Mo and Re to seawater (the riverine flux), and the oxic and anoxic fluxes of Mo and Re out of seawater.

The inventories of Mo and Re in modern seawater are,

$$
\begin{aligned}
\text { Mo }_{S W} & =[\mathrm{Mo}]_{S W} * \text { volume }_{S W} & \text { Re }_{S W} & =\left[\text { Re }_{S W} * \text { volume }_{S W}\right. \\
& =107 \frac{\text { nmol }}{\mathrm{L}} * 1.35 \times 10^{21} \mathrm{~L} & & =40 \frac{\mathrm{pmol}^{\mathrm{mol}}}{\mathrm{L}} * 1.35 \times 10^{21} \mathrm{~L} \\
& =1.45 \times 10^{14} \mathrm{~mol} & & =5.40 \times 10^{10} \mathrm{~mol}
\end{aligned}
$$

The riverine ( riv) fluxes of Mo and Re to seawater (in) are (we assume a preanthropogenic Re concentration for world rivers),

$$
\begin{aligned}
f \mathrm{Mo}_{\text {in }} & =[\mathrm{Mo}]_{\text {riv }} * f \mathrm{H}_{2} \mathrm{O}_{\text {riv }} & f R e_{\text {in }} & =[R e]_{\text {riv }} * f \mathrm{H}_{2} \mathrm{O}_{\text {riv }} \\
& =8.0 \frac{\mathrm{nmol}}{\mathrm{kg}} * 3.86 \times 10^{11} \frac{\mathrm{kg}}{\mathrm{yr}} & & 11.2 \frac{\mathrm{pmol}}{\mathrm{kg}} * 3.86 \times 10^{11} \frac{\mathrm{kg}}{\mathrm{yr}} \\
& =3.09 \times 10^{8} \frac{\mathrm{mol}}{\mathrm{yr}} & & =4.32 \times 10^{5} \frac{\mathrm{mol}}{\mathrm{yr}}
\end{aligned}
$$


Oxic (ox) and anoxic (anox) fluxes of Mo and Re from seawater (out) are,

$$
\begin{aligned}
& f M o_{\text {out ox }}=3.09 \times 10^{8} \frac{\mathrm{mol}}{\mathrm{yr}} * 0.7 \\
& =2.16 \times 10^{8} \frac{\mathrm{mol}}{\mathrm{yr}} \\
& f e_{\text {out ox }}=4.32 \times 10^{5} \frac{\mathrm{mol}}{\mathrm{yr}} * 0.7 \\
& =3.03 \times 10^{5} \frac{\mathrm{mol}}{\mathrm{yr}} \\
& \text { and } \\
& \text { and } \\
& f \text { Mout anox }_{\text {on }}=3.09 \times 10^{8} \frac{\mathrm{mol}}{\mathrm{yr}} * 0.3 \\
& =9.26 \times 10^{7} \frac{\mathrm{mol}}{\mathrm{yr}} \\
& f \operatorname{Re}_{\text {out anox }}=4.32 \times 10^{5} \frac{\mathrm{mol}}{\mathrm{yr}} * 0.3 \\
& =1.30 \times 10^{5} \frac{\mathrm{mol}}{\mathrm{yr}}
\end{aligned}
$$

Assuming that fluxes from seawater are first-order (see assumption 3, e.g. $f M o_{\text {out ox }} \propto$ $M o_{S W}$ ), we can solve for the constants of Mo and Re oxic and anoxic flux rates. For example, for $k_{\text {Mo out ox }}$,

$$
\begin{aligned}
\frac{d M o_{S W}}{d t} & \propto M o_{S W} \\
\frac{d M o_{S W}}{d t} & =-k M o_{S W} \\
\frac{d M o_{S W}}{M o_{S W}} & =-k d t \\
\int \frac{d M o_{S W}}{M o_{S W}} & =\int-k d t \\
\log _{e} M o_{S W} & =-k t+C
\end{aligned}
$$


at $t=0, M o_{S W}$,

$$
\begin{aligned}
\log _{e} M o_{S W 0} & =-k(0)+C \\
\log _{e} M o_{S W} 0 & =C \\
\text { so } & \\
\log _{e} M o_{S W} & =-k t+\log _{e} M o_{S W} 0 \\
\log _{e} M o_{S W}-\log _{e} M o_{S W} 0 & =-k t \\
\log _{e}\left(\frac{M o_{S W}}{M o_{S W} 0}\right) & =-k t \\
\frac{M o_{S W}}{M o_{S W 0}} & =e^{-k t} \\
M o_{S W} & =M o_{S W 0} * e^{-k t}
\end{aligned}
$$

The flux of Mo out of the oceans over a given time-period is,

$$
\begin{aligned}
f M o_{\text {out }} & =M o_{S W}-M o_{S W} \\
& =M o_{S W}-M o_{S W} * e^{-k t} \\
& =M o_{S W} 0\left(1-e^{-k t}\right)
\end{aligned}
$$

allowing us to solve for $k$. 


$$
\begin{aligned}
f M o_{\text {out }} & =M o_{S W 0}\left(1-e^{-k t}\right) \\
\frac{f M o_{\text {out }}}{M o_{S W} 0} & =1-e^{-k t} \\
1-\frac{f M o_{\text {out }}}{M o_{S W} 0} & =e^{-k t} \\
\log _{e}\left(1-\frac{f M o_{\text {out }}}{M o_{S W 0}}\right) & =-k t \\
-\log _{e}\left(1-\frac{f M o_{\text {out }}}{M o_{S W 0}}\right) * \frac{1}{t} & =k
\end{aligned}
$$

Therefore, for any annual flux out of seawater (e. g. $f M o_{\text {out ox }}$ ),

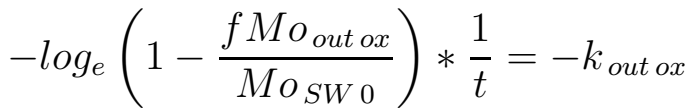

$$
\begin{aligned}
& -\log _{e}\left(1-\frac{2.16 \times 10^{8} \frac{\mathrm{mol}}{\mathrm{yr}}}{1.45 \times 10^{14} \mathrm{~mol}}\right) * \frac{1}{1 y r}=k_{\text {Moout ox }} \\
& 1.490 \times 10^{-6} \mathrm{yr}^{-1}=k_{\text {Mo out ox }}
\end{aligned}
$$

The four flux constants are therefore:

$$
\begin{aligned}
k_{\text {Mo out ox }} & =1.490 \times 10^{-6} \mathrm{yr}^{-1} \\
k_{\text {Moout anox }} & =6.386 \times 10^{-7} \mathrm{yr}^{-1} \\
k_{\text {Re out ox }} & =5.611 \times 10^{-6} \mathrm{yr}^{-1} \\
k_{\text {Re out anox }} & =2.407 \times 10^{-7} \mathrm{yr}^{-1}
\end{aligned}
$$


A box model of changing Mo and Re seawater inventories with with time due to changing anoxic fluxes can now be constructed. The effects of the seawater response times and changing sink fluxes on seawater inventories of Mo and Re are evaluated.

Response times based on previous (Bertine and Turekian, 1973; Colodner ET AL., 1993a) and revised (this study) world river average values will be compared.

Metal source fluxes are kept constant (assumption 2) while sink fluxes alternate between two scenarios (Fig 2-12): one equivalent to the modern Mo sink flux proportions (70\% oxic, 30\% anoxic; SieBerT ET AL., 2003; Re sink flux proportions are assumed to be identical), the other with an anoxic sink flux that is twice the modern value.

For a given year, $t(i)$, the metal inventories in seawater are given by,

$$
\begin{aligned}
& \operatorname{Mo}_{S W}(i)=M o_{S W}(i-1)+f M o_{\text {in }}-f M o_{\text {out }}, \\
& \text { and } \\
& \operatorname{Re}_{S W}(i)=\operatorname{Re}_{S W}(i-1)+f R e_{\text {in }}-f \operatorname{Re}_{\text {out }},
\end{aligned}
$$

where $f M o_{\text {in }}, f R e_{\text {in }}$ and $f M o_{\text {out }}, f R e_{\text {out }}$ are metal fluxes into and out of seawater given by, 


$$
\begin{aligned}
& f M o_{\text {in }}=[M o]_{\text {riv }} *(t(i)-t(i-1)) \\
& f R e_{i n}=[R e]_{r i v} *(t(i)-t(i-1)) \\
& \text { and } \\
& f M o_{\text {out }}=f M o_{\text {out ox }}+f M o_{\text {out anox }} \\
& =\left(\operatorname{Mo}_{S W}(i-1) *\left(1-e^{-k_{\text {Mo out ox }} *(i-(i-1))}\right)\right) \\
& +\left(\operatorname{Mo}_{S W}(i-1) *\left(1-e^{-k_{\text {Mo out anox }} * i-(i-1)}\right)\right) \\
& f R e_{\text {out }}=f R e_{\text {out ox }}+f R e_{\text {out anox }} \\
& =\left(\operatorname{Re}_{S W}(i-1) *\left(1-e^{-k_{\text {Re out ox } * i-(i-1)}}\right)\right) \\
& +\left(\operatorname{Re}_{S W}(i-1) *\left(1-e^{-k_{\text {Re out anox } * i-(i-1)}}\right)\right)
\end{aligned}
$$


Scenario 1
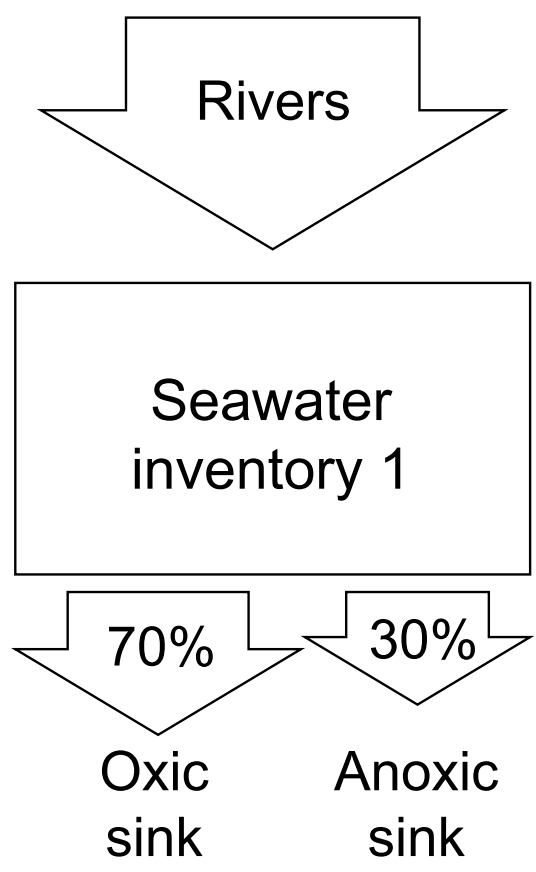

Scenario 2
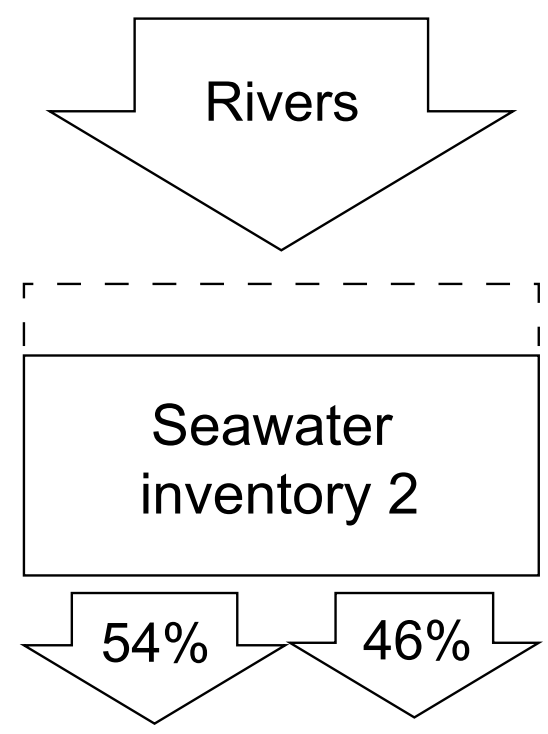

Oxic sink

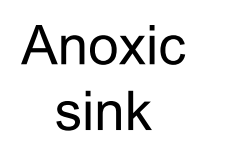

Figure 2-12: Scenarios considered in the modeling of Mo and Re inventories. Scenario 1 is the modern situation in which the oxic and anoxic sinks respectively account for $70 \%$ and $30 \%$ of the total sink flux for Mo (SIEBERT ET AL., 2003). In scenario 2 the flux to the oxic sink is held constant while the flux to the anoxic sink is doubled; the two sinks now account for $54 \%$ and $46 \%$ of the total Mo sink, respectively. Note that this model yields oxic and anoxic sink proportions of $30 \%$ and $70 \%$, equivalent to those determined for the Mid-Proterozoic (ARNOLD ET AL., 2004), at anoxic fluxes 5.45 times greater than the observed modern value. 


\section{Bibliography}

Algeo, T. J. (2004), Can marine anoxic events draw down the trace element inventory of seawater?, Geology, 32: 1057-1060. [15, 91, 92, 175, 199]

Algeo, T. J. and Lyons, T. W. (2006), Mo-total organic carbon in modern anoxic marine environments: Implications for analysis of paleoredox and paleohydrographic conditions, Paleoceanography, 21: 23 pp. [16, 22, 23, 75, 176]

Anbar, A. D., Creaser, R. A., Papanastassiou, D. A., and Wasserburg, G. J. (1992), Rhenium in seawater: confirmation of generally conservative behavior, Geochimica et Cosmochimica Acta, 56: 4099-4103. [22, 23, 26, 79, 89, 91]

Anbar, A. D. and Knoll, A. H. (2002), Proterozoic ocean chemistry and evolution: a bioinorganic bridge?, Science, 297: 1137-1142. [22]

Arne, D. C., Bierlein, F. P., Morgan, J. W., and Stein, H. J. (2001), Re-Os dating of sulfides associated with gold mineralization in central Victoria, Australia, Economic Geology, 96: 1455-1459. [78]

Arnold, G. L., Anbar, A. D., Barling, J., and Lyons, T. W. (2004), Molybdenum isotope evidence for widespread anoxia in mid-Proterozoic oceans, Science, 304: 87-90. [16, 22, 98, 104, 129, 175, 176, 186, 198, 199]

Audry, S., Blank, G., Schäfer, J., and Robert, S. (2007), Effect of estuarine sediment resuspension on early diagenesis, sulfide oxidation and dissolved molybdenum and uranium distribution in the Gironde estuary, France, Chemical Geology, 238: 149167. [79]

Barra, F., Ruiz, J., Mathur, R., and Titley, S. (2003), A Re-Os study of sulfide minerals from the Bagdad porphyry $\mathrm{Cu}-\mathrm{Mo}$ deposit, northern Arizona, USA, Mineralium Deposita, 38: 585-596. [78, 151] 
Benoit, G., Wang, E. X., Nieder, W. C., Levandowski, M., and Breslin, V. (1999), Sources and history of heavy metal contamination and sediment deposition in Tivoli South Bay, Hudson River, New York, Estuaries, 2A: 167-178. [87]

Berner, R. A. (1971), Worldwide sulfur pollution in rivers, Journal of Geophysical Research, 76: 6597-6600. [73]

Berner, R. A. and Berner, E. K. (1987), The Global Water Cycle, Prentice-Hall, 397 pp. $[60,73]$

Berner, R. A., Lasaga, A. C., and Garrels, R. M. (1983), The carbonate-silicate geochemical cycle and its effects on atmospheric carbon dioxide over the last 100 million years, American Journal of Science, 283: 641-683. [15, 21]

Berrang, P. G. and Grill, E. V. (1974), The effect of manganese oxide scavenging on molybdenum in Saanich Inlet, British Columbia, Marine Chemistry, 2: 125-148. [79]

Bertine, K. K. and Goldberg, E. D. (1971), Fossil fuel combustion and the major sedimentary cycle, Science, 173: 233-235. [72]

Bertine, K. K. and Turekian, K. K. (1973), Molybdenum in marine deposits, Geochimica et Cosmochimica Acta, 37: 1415-1434. [15, 16, 21, 23, 24, 26, 65, 69, 70, 71, $74,76,91,94,95,102,197]$

Berzina, A. N., Sotnikov, V. I., Economou-Eliopoulos, M., and Eliopoulos, D. G. (2005), Distribution of rhenium in molybdenite from porphyry $\mathrm{Cu}-\mathrm{Mo}$ and $\mathrm{Mo}-$ $\mathrm{Cu}$ deposits of Russia (Siberia) and Mongolia, Ore Geology Reviews, 26: 91-113. $[78,151]$

Brookins, D. P. (1986), Rhenium as analog for fissiogenic technetium: Eh-pH diagram $\left(25^{\circ} \mathrm{C}, 1\right.$ bar) constraints, Applied Geochemistry, 1: 513-517. [22] 
Brügmann, G. E., Birck, J. L., Herzig, P. M., and Hofmann, A. W. (1998), Os isotopic composition and Os and Re distributions in the active mound of the TAG hydrothermal system, Mid-Atlantic Ridge, Proceedings of the Ocean Drilling Program, Scientific Results, 158: 91-100. [78]

Brumsack, H.-J. (2006), The trace metal content of recent organic carbon-rich sediments: Implications for Cretaceous black shale formation, Palaeogeography, Palaeoclimatology, Palaeoecology, 232: 344-361. [78]

Calvert, S. E. and Pederson, T. F. (1993), Geochemistry of Recent oxic and anoxic marine sediments: Implications for the geological record, Marine Geology, 113: 67-88. [21]

Capitant, M., Francotte, J., Picot, P., and Troly, G. (1963), Hautes teneurs de rhénium dans une molybdénite de Kipushi, Comptes Rendus de l'Académie des Sciences, 257: 3443-3444. [75, 150]

Chang, T. (1998), Regeneration industry helps refiners control costs and limit liabilities, Oil and Gas Journal, 96: 49-54. [72]

Chappaz, A., Gobeil, C., and Tessier, A. (2008), Sequestration mechanisms and anthropogenic inputs of rhenium in sediments from Eastern Canada lakes, Geochimica et Cosmochimica Acta, 72: 6027-6036. [72]

Cohen, A. S., Coe, A. L., Bartlett, J. M., and Hawkesworth, C. J. (1999), Precise Re-Os ages of organic-rich mudrocks and the Os isotopic composition of Jurassic seawater, Earth and Planetary Science Letters, 167: 159-173. [76, 78, 150, 151]

Collier, R. W. (1985), Molybdenum in the Northeast Pacific Ocean, Limnology and Oceanography, 30: 1351-1354. [22, 23, 26, 79, 89, 91]

Colodner, D., Edmond, J., and Boyle, E. (1995), Rhenium in the Black Sea: com- 
parison with molybdenum and uranium, Earth and Planetary Science Letters, 131: $1-15 .[23,72,91]$

Colodner, D., Sachs, J., Ravizza, G., Turekian, K., Edmond, J., and Boyle, E. (1993a), The geochemical cycle of rhenium: a reconnaissance, Earth and Planetary Science Letters, 117: 205-221. [15, 16, 21, 22, 23, 24, 26, 65, 69, 70, 71, 76, 79, 86, 87, 91, 92, 94, 95, 96, 102, 150, 151, 197, 198]

Colodner, D. C., Boyle, E. A., and Edmond, J. M. (1993b), Determination of rhenium and platinum in natural waters and sediments, and iridium in sediments by flow injection isotope dilution inductively coupled plasma mass spectrometry, Analytical Chemistry, 65: 1419-1425. [27]

Corliss, J. B., Dymond, J., Gordon, L. I., Edmond, J. M., von Herzen, R. P., Ballard, R. D., Green, K., Williams, D., Bainbridge, A., Crane, K., and van Andel, T. H. (1979), Submarine thermal springs on the Galápagos rift, Science, 203: 1073-1083. $[87]$

Craddock, P. R. (2008), Geochemical Tracers of Processes Affecting the Formation of Seafloor Hydrothermal Fluids and Deposits in the Manus Back-Arc Basin, Ph.D. thesis, Massachussetts Institute of Technology / Woods Hole Oceanographic Institution. $[30,59,88]$

Creaser, R. A., Sannigrahi, P., Chacko, T., and Selby, D. (2002), Further evaluation of the Re-Os geochronometer in organic-rich sedimentary rocks: A test of hydrocarbon maturation effects in the Exshaw Formation, Western Canada Sedimentary Basin, Geochimica et Cosmochimica Acta, 66: 3441-3452. [78, 150, 151, 181, 186]

Crusius, J., Calvert, S., Pederson, T., and Sage, D. (1996), Rhenium and molybdenum enrichments in sediments as indicators of oxic, suboxic and sulfidic conditions of deposition, Earth and Planetary Science Letters, 145: 65-78. [15, 17, 21, 22, 75, $92,176,186,198]$ 
Dalai, T. K., Nishimura, K., and Nozaki, Y. (2005), Geochemistry of molybdenum in the Chao Phraya River estuary, Thailand: Role of suboxic diagenesis and porewater transport, Chemical Geology, 218: 189-202. [79]

Dalai, T. K., Singh, S. K., Trivedi, J. R., and Krishnaswami, S. (2002), Dissolved rhenium in the Yamuna River System and the Ganga in the Himalaya: role of black shale weathering on the budgets of $\mathrm{Re}$, Os, and $\mathrm{U}$ in rivers and $\mathrm{CO}_{2}$ in the atmosphere, Geochimica et Cosmochimica Acta, 66: 29-43. [76, 87, 95, 150, 151]

de Vries, M. P. and Weiss, L. A. (2001), Salt-front movement in the Hudson River estary, New York - simulations by one-dimensional flow and solute-transport models, Tech. rep., United States Geological Survey. [86]

Duyck, C., Miekeley, N., Porto da Silveira, C. L., and Szatmari, P. (2002), Trace element determination in crude oil and its fractions by inductively coupled plasma mass spectrometry using ultrasonic nebulization of toluene solutions, Spectrochimica Acta, 57B: 1979-1990. [72]

Edmond, J. M., Measures, C., McDuff, R. E., Chan, L. H., Collier, R., Grant, B., Gordon, L. I., and Corliss, J. B. (1979), Ridge crest hydrothermal activity and the balances of the major and minor elements in the ocean: The Galapagos data, Earth and Planetary Science Letters, 46: 1-18. [87]

Elderfield, H. and Schultz, A. (1996), Mid-ocean ridge hydrothermal fluxes and the chemical composition of the ocean, Annual Review of Earth and Planetary Sciences, 24: 191-224. [23, 87, 90]

Emerson, S. R. and Huested, S. S. (1991), Ocean anoxia and the concentrations of molybdenum and vanadium in seawater, Marine Chemistry, 34: 177-196. [15, 92, 199]

Esser, B. K. and Turekian, K. K. (1993), The osmium isotopic composition of the 
continental crust, Geochimica et Cosmochimica Acta, 57: 3093-3104. [73, 149, 151, $176,184]$

Evans, H. T. J., Manheim, F. T., and Landerren, S. (1978), Molybdenum, in Wedepohl, K. H., Correns, C. W., Shaw, D. M., Turekian, K. K., and Zemann, J. (eds.), Handbook of Geochemistry II-4, Handbook of Geochemistry, vol. II, chap. 42, Springer-Verlag, 42A1-42O2. [72, 74, 75, 76, 91]

Fekete, B. M., Vörösmarty, and Grabs, W. (2002), High-resolution fields of global runoff combining observed river discharge and simulated water balances, Global Biogeochemical Cycles, 16. [23, 24, 60, 65, 71, 74, 90]

Feng, H., Cochran, J. K., Lwiza, H., Brownawell, B. J., and Hirschberg, D. J. (1998), Distribution of heavy metal and PCB contaminants in the sediments of an urban estuary: the Hudson River, Marine Environmental Research, 45: 69-88. [87]

Fleischer, M. (1959), The geochemistry of rhenium, with special reference to its occurrence in molybdenite, Economic Geology, 55: 607-609. [75, 78, 150, 151]

Fleischer, M. (1960), The geochemistry of rhenium-addendum, Economic Geology, 55: $607-609$. [78, 150]

Fleischer, M. (1963), New mineral names, American Mineralogist, 1948: 209-217. [75]

Fouquet, Y., von Stackelberg, U., Charlou, J. L., Donval, J. P., Foucher, J. P., Erzinger, J., Herzig, P., Mühe, R., Wiedicke, M., Soakai, S., and Whitechurch, H. (1991), Hydrothermal activity in the Lau back-arc basin: Sulfides and water chemistry, Geology, 19: 303-306. [87]

Freydier, C., Ruiz, J., Chesley, J., McCandless, T., and Munizaga, F. (1997), Re-Os isotope systematics of sulfides from felsic igneous rocks: application to base metal porphyry mineralizations in Chile, Geology, 25: 775-778. [78] 
Fukai, R. and Meinke, W. W. (1962), Activation analyses of vanadium, arsenic, molybdenum, tungsten, rhenium, and gold in marine organisms, Limnology and Oceanography, 7: 186-200. [22]

Graham, S. T., Famiglietti, J. S., and Maidment, D. R. (1999), Five-minute, 1/2º, and $1^{\circ}$ data sets of continental watersheds and river networks for use in regional and global hydrologica and climate system modelin studies, Water Resources Research, 35: 583-587. [39, 67, 95]

Hannah, J. L., Bekker, A., Stein, H. J., Markey, R. J., and Holland, H. D. (2004), Primitive Os and $2316 \mathrm{Ma}$ age for marine shale: implications for Paleoproterozoic glacial events and the rise of atmospheric oxygen, Earth and Planetary Science Letters, 225: 43-52. [78]

Hauri, E. H. and Hart, S. R. (1997), Rhenium abundances and systematics in oceanic basalts, Chemical Geology, 139: 185-205. [73, 149, 176, 184]

Head, P. C. and Burton, J. D. (1970), Molybdenum in some ocean and estuarine waters, Journal of the Marine Biological Association of the UK, 50: 439-448. [79]

Hodge, V. F., Johannesson, K. H., and Stetzenbach, K. J. (1996), Rhenium, molybdenum, and uranium in groundwater from the southern Great Basin USA: evidence for conservative behavior, Geochimica et Cosmochimica Acta, 60: 3197-3214. [73, $78]$

Jaffe, L. A., Peucker-Ehrenbrink, B., and Petsch, S. T. (2002), Mobility of rhenium, platinum group elements and organic carbon during black shale weathering, Earth and Planetary Science Letters, 198: 339-353. [17, 21, 76, 176, 177, 178, 179, 181, $183,186,198]$

Jones, B. and Manning, D. A. C. (1994), Comparison of geochemical indices used for the interpretation of palaeoredox conditions in ancient mudstones, Chemical Geology, 111: 111-129. [22] 
Kharkar, D. P., Turekian, K. K., and Bertine, K. K. (1968), Stream supply of dissolved silver, molybdenum, antimony, selenium, chromium, cobalt, rubidium and cesium to the oceans, Geochimica et Cosmochimica Acta, 32: 285-298. [26]

Kirk, J., Ruiz, J., Chesley, J., Titley, S., and Walshe, J. (2001), A detrital model for the origin of gold and sulfides in the Witwatersrand basin based on Re-Os isotopes, Geochimica et Cosmochimica Acta, 65: 2149-2159. [78]

Kirk, J., Ruiz, J., Chesley, J., Walshe, J., and England, G. (2002), A major Archean, gold- and crust-forming event in the Kaapvaal Craton, South Africa, Science, 297: 1856-1858. [78]

Klinkhammer, G. P. and Palmer, M. R. (1991), Uranium in the oceans: Where it goes and why, Geochimica et Cosmochimica Acta: 1799-1806. [21]

Koide, M., Hodge, V., Yang, J. S., and Goldberg, E. D. (1987), Determination of rhenium in marine waters and sediments by graphite furnace atomic absorption spectrometry, Analytical Chemistry, 59: 1802-1805. [23]

Koide, M., Hodge, V. F., Yang, J. S., Stallard, M., Goldberg, E. G., Calhoun, J., and Bertine, K. K. (1986), Some comparative marine chemistries of rhenium, gold, silver and molybdenum, Applied Geochemistry, 1: 705-714. [17, 75, 76, 88, 176, $179,198,199]$

Korzhinski, M. A., Tkachenko, S. I., Shmulovich, K. I., Taran, Y. A., and Steinberg, G. S. (1994), Discovery of pure rhenium mineral at Kudriavy volcano, Nature, 369: 51-52. $[75,150,151]$

Kucha, H. (1990), Geochemistry of the Kupferschiefer, Poland, International Journal of Earth Sciences, 79: 387-399. [73]

Łetowski, F., Serkies, J., and Niemec, J. (1966), Application of potential-pH diagrams 
for determination of the occurrence forms of trace elements in some economic mineral deposits, Economic Geology, 61: 1272-1279. [22]

Levinson, A. A. (1974), Introduction to Exploration Geochemistry, Calgary, Applied Publisher, 924 pp. [79]

Leybourne, M. I. and Cameron, E. M. (2008), Source, transport, and fate of rhenium, selenium, molybdenum, arsenic, and copper in groundwater associated with porphyry-Cu deposits, Atacama Desert, Chile, Chemical Geology, 247: 208-228. $[73,78]$

Li, X., Wai, O. W. H., Coles, J., Barry, Ramsey, M. H., and Thornton, I. (2000), Heavy metal distribution in sediment profiles of the Pearl River estuary, south China, Applied Geochemistry, 15: 567-581. [87]

Ling, H.-F., Gao, J. F., Zhao, K.-D., Jiang, S.-Y., and Ma, D. S. (2005), Comment on "Molybdenum isotope evidence for widespread anoxia in Mid-Proterozoic oceans", Science, 309: 1017c. [93]

Liu, G., Chou, C.-L., and Peng, Z. (2008), Abundances and isotopic compositions of rhenium and osmium in pyrite samples from Huaibei coalfield, Anhui, China, International Journal of Earth Sciences, 97: 617-621. [78]

Livingstone, D. A. (1963), Chemical composition of rivers and lakes, in Fleischer, M. (ed.), Data of Geochemistry, 6th edn., chap. G: Chemical Composition of Rivers and Lakes, United States Geological Survey Professional Paper, United States Government, Department of the Interior, G1-G64. [60, 62, 67, 69, 95]

Mao, J., Du, A., Seltmann, R., and Yu, Y. (2003), Re-Os ages for the Shamika porphyry Mo deposit and the Lipovy Log rare metal pegmatite, central Urals, Russia, Mineralium Deposita, 38: 251-257. [78, 151] 
Mao, J., Wang, Y., Lehmann, B., Yu, Y., Du, A., Mei, Y., Li, Y., Zang, W., Stein, H. J., and Zhou, T. (2006), Molybdenite Re - Os and albite ${ }^{40} \mathrm{Ar} /{ }^{39} \mathrm{Ar}$ dating of $\mathrm{Cu}-\mathrm{Au}-\mathrm{Mo}$ and magnetite porphyry systems in the Yangtze River valley and metallogenic implications, Ore Geology Reviews, 29: 307-324. [78, 151]

Markey, R., Stein, H., and Morgan, J. (1998), Highly precise Re-Os dating for molybdenite using alkaline fusion and NTIMS, Talanta, 45: 935-946. [78, 151]

Mason, B. and Moore, C. B. (1982), Principles of Geochemistry, Wiley Eastern, 344 pp. $[77,79]$

Mathur, R., Ruiz, J., and Munizaga, F. (2000), Relationship between copper tonnage of Chilean base-metal porphyry deposits and Os isotope ratios, Geology, 28. [78]

Mathur, R., Ruiz, J., and Tornos, F. (1999), Age and sources of the ore at Tharsis and Rio Tinto, Iberian Pyrite Belt, from Re-Os isotopes, Mineralium Deposita, 34: 790-793. [78]

Mathur, R., Titley, S., Ruiz, J., Gibbins, S., and Friehauf, K. (2005), A Re - Os study of sedimentary rocks and copper - gold ores from Ertsberg District, West Papua, Indonesia, Ore Geology Reviews, 26. [75, 78]

McCandless, T. E., Ruiz, J., and Campbell, A. R. (1993), Rhenium behavior in molybdenite in hypogene and near surface environments: implications for Re-Os geochronomtery, Geochimica et Cosmochimica Acta, 57: 889-905. [76, 78, 151]

McLennan, S. M. (2001), Relationships between the trace element composition of sedimentary rocks and upper continental crust, Geochemistry, Geophysics, Geosystems, 2: doi:10.1029/2000GC000109. [71, 74, 77, 79, 176]

Meisel, T. and Stotter, V. (2007), Identifying the sources of PGE, Re and Sb in road dust and soils along highways, Geochimica et Cosmochimica Acta Supplement, 71: A650. [72] 
Menard, H. W. and Smith, S. M. (1966), Hypsometry of ocean basin provinces, Journal of Geophysical Research, 71: 4305-4325. [23, 91]

Metz, S. and Trefry, J. H. (2000), Chemical and mineralogical influences on concentrations of trace metals in hydrothermal fluids, Geochimica et Cosmochimica Acta, 64: 2267-2279. [23, 87, 90]

Meybeck, M. (1979), Concentration des eaux fluviales en éléments majeurs et apports en solution aux océans, Revue de Géologie Dynamique et de Géographie Physique, 21: 215-246. [60, 67, 69, 73, 74, 95]

Meybeck, M. (1988), Physical and Chemical Weathering in Geochemical Cycles, chap. 12 How to establish and use world budgets of riverine material, Kluwer Academic Publishers, 247-272. [73]

Meybeck, M. and Helmer, R. (1989), The quality of rivers: from pristine stage to global pollution, Palaeogeography, Palaeoclimatology, Palaeoecology, 75: 283-309. $[60,62,74]$

Meybeck, M. and Ragu, A. (1995), River discharges to the oceans: An assessment of suspended solids, major ions and nutirents, Tech. rep., United Nations Environment Programme. [24, 60, 61, 67, 69, 95]

Miller, C. A. (2004), Re-Os dating of algal laminites: reduction enrichment of metals in the sedimentary environment and new geoporphyrins, Master's thesis, University of Saskatchewan. [76, 189]

Miller, C. A., Peucker-Ehrenbrink, B., and Ball, L. (2008), Rhenium isotopes in natural systems, Geochimica et Cosmochimica Acta, 72: A628. [22, 93]

Millero, F. J. and Poisson, A. (1981), International one-atmosphere equation of state for seawater, Deep-Sea Research, 28A: 625-629. [23, 91] 
Mitchell, R. H., Laflamme, J. H. G., and Cabri, L. J. (1989), Rhenium sulphide from the Coldwell complex, northwestern Ontario, Canada, Mineralogical Magazine, 53: 635-637. [75, 150]

Montgomery, R. B. (1958), Water characteristics of Atlantic Ocean and of world ocean, Deep-Sea Research, 5: 134-148. [23, 91]

Morachevskii, D. E. and Nechaeva, A. A. (1960), Characteristics of migration of rhenium from molybdenites, Geochemistry, 6: 648-651. [76, 78, 150, 151]

Morelli, R. M., Creaser, R. A., Selby, D., Kontak, D. J., and Horne, R. J. (2005), Rhenium-osmium geochronology of arsenopyrite in Meguma Group gold deposits, Meguma terrane, Nova Scotia, Canada: evidence for multiple gold-mineralizing events, Economic Geology, 100: 1229-1242. [75, 78]

Morelli, R. M., Creaser, R. A., Seltmann, R., Stuart, F. M., Selby, D., and Graupner, T. (2007), Age and source constraints for the giant Muruntau gold deposit, Uzbekistan, from coupled Re-Os-He isotopes in arsenopyrite, Geology, 35: 795-798. [78]

Morford, J. L. and Emerson, S. (1999), The geochemistry of redox sensitive trace metals in sediments, Geochimica et Cosmochimica Acta, 63: 1735-1750. [15, 16, $17,21,22,23,75,91,92,96,175,198,200]$

Morford, J. L., Martin, W. R., Kalnejais, L. H., François, R., Bothner, M., and Karle, I.-M. (2007), Insights on geochemical cycling of U, Re and Mo from seasonal sampling in Boston Harbor, Massachusetts, USA, Geochimica et Cosmochimica Acta, 71: 895-917. [21]

Morgan, J. W., Golightly, D. W., and Dorrzapf, A. F. (1991), Methods for the separation of rhenium, osmium and molybdenum applicable to isotope geochemistry, Talanta, 38: 259-265. [26] 
Morris, A. W. (1975), Dissolved molybdenum and vanadium in the northeast Atlantic Ocean, Deep-Sea Research, 22: 49-54. [22, 23, 26, 79, 91]

Morris, D. F. C. and Short, E. L. (1966), Minerals of rhenium, Mineralogical Magazine, 35: 871-873. [75, 150]

Moyse, B. M. (2000), Process, catalyst choices key to producing 30-ppm sulfur fuels, Oil and Gas Journal, 98: 72-74. [72]

Nägler, T. F., Siebert, C., Lüschen, H., and Böttcher, M. E. (2005), Sedimentary Mo isotope record across the Holocene fresh-brackish water transition of the Black Sea, Chemical Geology, 219: 283-295. [22]

Neubert, N., Nägler, T. F., and Böttcher, M. E. (2008), Sulfidity controls molybdenum isotope fractionation into euxinic sediments: Evidence from the modern Black Sea, Geology, 36: 775-778. [16, 22, 186]

Newberry, R. J. J. (1979), Polytypism in molybdenite (II): relationships between polytypism, ore deposition/alteration stages and rhenium contents, American Mineralogist, 64: 768-775. [76]

Noddack, I. and Noddack, W. (1931), Die Geochemie des Rheniums, Zeitschrift für Physikalische Chemie, 16: 207-244. [74, 78, 149, 151]

Noddack, V. W., Tacke, I., and Berg, V. O. (1925), Zwei neue Elemente der Mangangruppe, Sitzung der physikalisch-mathematischen Klasse, 11: 400-409. [74, 75, 149]

Pearce, C. R., Cohen, A. S., Coe, A. L., and Burton, K. W. (2008), Molybdenum isotope evidence for global ocean anoxia coupled with perturbations to the carbon cycle during the Early Jurassic, Geology, 36: 231-234. [16, 93, 98, 175, 199] 
Peterson, R. G., Hamilton, J. C., and Myers, A. T. (1959), An occurrence of rhenium associated with uraninite in Coconino County, Arizona, Economic Geology, 54: 254-267. [75]

Peucker-Ehrenbrink, B. and Hannigan, R. E. (2000), Effects of black shale weathering on the mobility of rhenium and platinum group elements, Geology, 28: 475-478. $[17,76,78,150,151,176,179,198]$

Peucker-Ehrenbrink, B. and Miller, M. W. (2007), Quantitative bedrock geology of the continents and large-scale drainage regions, Geochemistry Geophysics Geosystems, 8: doi:10.1029/2006GC001544. [67]

Pierson-Wickmann, A.-C., Reisberg, L., and France-Lanord, C. (2003), Behavior of Re and Os during low-temperature alteration: results from Himalayan soils and altered black shales during low-temperature alteration: results from Himalayan soils and altered black shales, Geochimica et Cosmochimica Acta, 66: 1539-1548. $[17,76,87,176,179,198]$

Poplavko, E. M., Ivanov, V. V., Karasik, T. G., Miller, A. D., Orekhov, V. S., D., T. S., Tarkhov, Y. A., and Fadeyeva, V. A. (1974), On the concentration of rhenium in petroleum, petroleum bitumens, and oil shales, Geochemistry International, 11: 969-972. [72]

Poulson, R. L., Siebert, C., McManus, J., and Berelson, W. M. (2006), Authigenic molybdenum isotope signatures in marine sediments, Geology, 34: 617-620. [16, $22,91,200]$

Power, M. R., Pirrie, D., Jedwab, J., and Stanley, C. J. (2004), Platinum-group element mineralization in an As-rich magmatic sulphide system, Talnotrie, southwest Scotland, Mineralogical Magazine, 68: 395-411. [75, 150]

Quinby-Hunt, M. and Wilde, P. (1996), Chemical deopositional environments of calcic marine black shales, Economic Geology, 91: 4-13. [21] 
Raiswell, R. and Plant, J. (1980), The incorporation of trace elements into pyrite during diagenesis of black shales, Yorkshire, England, Economic Geology, 75: 684699. [76]

Ravizza, G., Martin, C. E., German, C. G., and Tompson, G. (1996), Os isotopes as tracers in seafloor hydrothermal systems: metalliferous deposits from the TAG hydrothermal area, $26^{\circ} \mathrm{N}$ Mid-Alantic Ridge, Earth and Planetary Science Letters, 138: 105-119. $[23,88]$

Ravizza, G. E. and Turekian, K. K. (1989), Application of the ${ }^{187}$ Re- ${ }^{187}$ Os system to black shale geochronometry, Geochimica et Cosmochimica Acta, 53: 3257-3262. $[15,78,150,151]$

Ravizza, G. E., Turekian, K. K., and Hay, B. J. (1991), The geochemistry of rhenium and osmium in recent sediments from the Black Sea, Geochimica et Cosmochimica Acta, 55: 3741-3752. [15, 75, 150, 151, 198]

Rodhe, H. (1992), Modeling biogeochemical cycles, in Butcher, S. S., Charlson, R. J., Orians, G. H., and Wolfe, G. V. (eds.), Global Biogeochemical Cycles, chap. 4, Academic Press, 55-72. [91]

Rosman, K. J. R. and Taylor, P. D. P. (1998), Isotopic compositions of the elements 1997, Pure and Applied Chemistry, 70: 217-235. [27, 126, 135]

Roy-Barman, M. and Allègre, C. J. (1994), ${ }^{187} \mathrm{Os} /{ }^{186} \mathrm{Os}$ ratios of mid-ocean ridge basalts and abyssal peridotites, Geochimica et Cosmochimica Acta, 58: 5043-5054. [88]

Selby, D. and Creaser, R. A. (2001), Re-Os geochronology and systematics in molybdenite from the Endako porphyry molybdenum deposit, British Columbia, Canada, Economic Geology, 96: 197-204. [78, 151] 
Selby, D., Creaser, R. A., and Fowler, M. G. (2005), Direct radiometric dating of hydrocarbon deposits using rhenium-osmium isotopes, Science, 308: 1293-1295. $[72]$

Selby, D., Creaser, R. A., and Fowler, M. G. (2007a), Re-Os elemental and isotopic systematics in crude oils, Geochimica et Cosmochimica Acta, 71: 378-386. [72]

Selby, D., Creaser, R. A., Stein, H. J., Markey, R. J., and Hannah, J. L. (2007b), Assessment of the ${ }^{187}$ Re decay constant by cross calibration of Re-Os molybdenite and $\mathrm{U}-\mathrm{Pb}$ zircon chronometers in magmatic ore systems, Geochimica et Cosmochimica Acta, 71: 1999-2013. [78, 153]

Seyfried, W. E. J. (1987), Experimental and theoretical constraints on hydrothermal alteration processes at mid-ocean ridges, Annual Review of Earth and Planetary Sciences, 15: 317-335. [89]

Seyfried, W. E. J. and Mottl, M. J. (1982), Hydrothermal alteration of basalt by seawater under seawater-dominated conditions, Geochimica et Cosmochimica Acta, 46: $985-1002$. [89]

Siebert, C., Nägler, T. F., von Blanckenburg, F., and Kramers, J. D. (2003), Molybdenum isotope records as a potential new proxy for paleoceanography, Earth and Planetary Science Letters, 211: 159-171. [15, 16, 17, 22, 76, 91, 92, 94, 98, 102, $104,176,179,186,199]$

Stein, H. J., Markey, R. J., Morgan, J. W., Hannah, J. L., and Scherstén, A. (2001), The remarkable Re-Os geochronometer in molybdenite: how and why it works, Terra Nova, 13: 479-486. [78]

Stein, H. J., Morgan, J. W., and Anders, S. (2000), Re-Os dating of low-level highly radiogenic (LLHR) sulfides: the Harnäs gold deposit, southwest Sweden, records continental-scale tectonic events, Economic Geology, 95: 1657-1671. [78] 
Stein, H. J., Sunblad, K., Markey, R. J., Morgan, J. W., and Motuza, G. (1998), Re-Os ages for Archean molybdenite and pyrite, Kuittila-Kivisuo, Finland and Proterozoic molybdenite, Kabeliai, Lithuania: testing the chronometer in a metamorphic and metasomatic setting, Mineralium Deposita, 33: 329-345. [75, 78]

Sugawara, K. and Okabe, S. (1961), Geochemistry of molybdenum in natural waters (II), Journal of Earth Sciences Nagoya University, 9: 114-128. [72]

Sun, W., Bennett, V. C., Eggins, S. M., Kamenetsky, V. S., and Arculus, R. J. (2003a), Enhanced mantle-to-crust rhenium transfer in undegassed arc magmas, Nature, 422: 294-297. [73, 176, 184]

Sun, W., Xie, Z., Chen, J., and Zhang, X. (2003b), Os-Os dating of copper and molybdenum deposits along the middle and lower reaches of the Yangtze River, China, Economic Geology, 98: 175-180. [78]

Takahashi, Y., Uruga, T., Suzuki, K., Tanida, H., Terada, Y., and Hattori, K. H. (2007), An atomic level study of rhenium and radiogenic osmium in molybdenite, Geochimica et Cosmochimica Acta, 71: 5180-5190. [75]

Taylor, S. R. and McLennan, S. M. (1985), The Continental Crust: its Composition and Evolution, Blackwell Scientific Publications, 312 pp. [79]

Terada, K., Osaki, S., Ishihara, S., and Kiba, T. (1971), Distribution of rhenium in molybdenites from Japan, Geochemical Journal, 4: 123-141. [78, 151]

Trefry, J. H., Butterfield, D. B., Metz, S., Massoth, G. J., Trocine, R. P., and Feely, A., Richard (1994), Trace metals in hydrothermal solutions from Cleft segment on the southern Juan de Fuca Ridge, Journal of Geophysical Research, 99: 4925-4935. $[87,89,90]$

Tribovillard, N., Algeo, T. J., Lyons, T., and Riboulleau, A. (2006), Trace metals as paleoredox and paleoproductivity proxies: an update, Chemical Geology. [21, 22] 
Tuit, C. B. (2003), The Marine Biogeochemistry of Molybdenum, Ph.D. thesis, Massachusetts Institute of Technology, Woods Hole Oceanographic Institution. [23]

Urey, H. C. (1952), The Planets, Their Origin and Development, Yale University Press, 245 pp. [15, 21]

Vistelius, A. B. and Sarmanov, O. V. (1967), Doklady Akademii Nauk USSR, 47: 4. [79]

Volborth, A., Tarkian, M., Stumpfl, E. F., and Housley, R. M. (1986), A survey of the Pd-Pt mineralization along the 35-km strike of the J-M Reef, Stillwater Complex, Montana, Canadian Mineralogist, 24: 329-346. [75]

Walker, L. M., Hays, P. B., and Kasting, J. F. (1981), A negative feedback mechanism for long-term stabilization of earth's surface temperature, Journal of Geophysical Research, 86: 9776-9782. [15, 21]

Wheat, C. G., Mottl, M. J., and Rudnicki, M. (2002), Trace element and REE composition of a low-temperature ridge-flank hydrothermal spring, Geochimica et Cosmochimica Acta, 66: 3693-3705. [23, 87, 88]

Whittaker, E. J. W. and Muntus, R. (1970), Ionic radii for use in geochemistry, Geochimica et Cosmochimica Acta, 34: 945-956. [75]

Woitke, P., Wellmitz, J., Helm, D., Kube, P., Lepom, P., and Litheraty, P. (2003), Analysis and assessment of heavy metal pollution in suspended solids and sediments of the river Danube, Chemosphere, 51: 633-642. [87]

Xiong, Y. and Wood, S. A. (1999), Experimental determination of the solubility of $\mathrm{ReO}_{2}$ and the dominant oxidation state of rhenium in hydrothermal solutions, Chemical Geology, 158: 245-256. [88] 
Xiong, Y. and Wood, S. A. (2001), Hydrothermal transport and deposition of rhenium under subcritical conditions (up to $200^{\circ} \mathrm{C}$ ) in light of experimental studies, Economic Geology, 96: 1429-1444. [88, 89]

Xiong, Y. and Wood, S. A. (2002), Experimental determination of the hydrothermal solubility of $\mathrm{ReS}_{2}$ and the $\mathrm{Re}-\mathrm{ReO}_{2}$ buffer assemblage and transport of rhenium under supercritical conditions, Geochemical Transactions, 3: 1-10. [88, 89]

Yang, J. S. (1991), High rhenium enrichment in brown algae: a biological sink of rhenium in the sea?, Hydrobiologia, 165: 165-170. [22]

Zhang, L., Xiao, W., Qin, K., Qu, W., and Du, A. (2005), Re-Os isotopic dating of molybdenite and pyrite in the Baishan Mo-Re deposit, eastern, Tianshan, NW China, and its geological significance, Mineralium Deposita, 39: 960-969. [78] 


\title{
Chapter 3
}

\section{Precise determination of rhenium}

\section{isotopic composition by}

\author{
multi-collector inductively-coupled
}

\section{plasma mass spectrometry}

\begin{abstract}
We present rhenium (Re) isotope data for commercially available Re standard solutions SRM 989, SRM 3143, and Alfa Aesar Specpure ${ }^{\circledR}$ Re, as well as two Re solutions made in-house from perrhenic acid and zone-refined flattened-wire ribbon from H. Cross Company. Data were generated by MC-ICPMS (Thermo Fisher Scientific NEPTUNE) using standard-bracketing combined with a tungsten (W) external correction. Standard reproducibility is $\pm 0.05 \%$ (2 s.d.) for analyte solution concen-

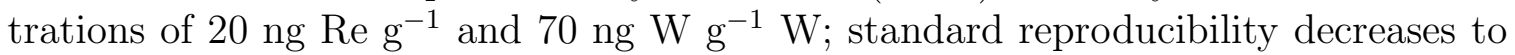
$\pm 0.1 \%$ (2 s.d.) when respective analyte concentrations are halved to $10 \mathrm{ng} \mathrm{Re} \mathrm{g}^{-1}$ and $35 \mathrm{ng} \mathrm{W} \mathrm{g}{ }^{-1}$. The five Re solutions exhibit an isotopic range of $0.29 \%$, providing the first evidence for stable isotope fractionation of Re. Data are also presented for the Devonian Ohio Shale standard USGS SDO-1; reproducibility of this natural material $( \pm 0.13 \%$, 2 s.d. $)$ is comparable to solution standard precisions at equivalent

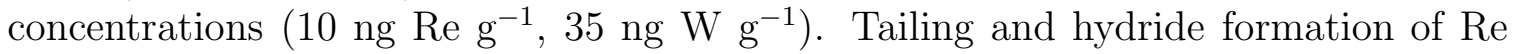
and $\mathrm{W}$ have negligible effects on measured Re isotopic composition. Analysis of geochemical materials introduces additional concerns. The low concentration of Re in most materials limits these analyses to Re-rich substrates. Isotopic fractionation oc-
\end{abstract}


curring during chromatographic purification is nullified by quantitative recovery, and the black shale sample matrix does not introduce Re isotope fractionation artifacts. Isobaric interference from radiogenic ${ }^{187}$ Os can be effectively removed by sparging sample solutions prior to analysis.

\subsection{Introduction and previous work}

Natural rhenium (Re, atomic number 75 ) is composed of two isotopes, ${ }^{185}$ Re and ${ }^{187} \mathrm{Re}$ with respective atomic abundances of $0.3740(2)$ and $0.6260(2)$, and masses of $184.9529557(30)$ amu and 186.9557508 (30) amu, giving an "average" atomic mass of 186.2067(3) amu and a ${ }^{187} \operatorname{Re} /{ }^{185} \operatorname{Re}$ of $1.6740(11)$ (Gramlich ET AL., 1973; AUdi And WAPstra, 1995; Rosman AND TAYlor, 1998). Isotope data presented herein are expressed as ${ }^{187} \mathrm{Re} /{ }^{185} \mathrm{Re}$, or in \%o using $\delta$-notation,

$$
\delta^{187} R e=\left[\left(\frac{\frac{187}{185} \operatorname{Re} \text { smpl }}{187 \text { Re } S R M 989_{\text {true }}}\right)-1\right] \times 1,000
$$

where NIST SRM 989 serves as a reference standard with ${ }^{187} \mathrm{Re} /{ }^{185} \mathrm{Re}$ of 1.6740 (Gramlich et AL., 1973).

Characterization of SRM 989 was the first absolute (i.e. gravimetric) isotope abundance determination for Re (GRAMLICH ET AL., 1973); it was done using positive thermal ionization mass spectrometry (PTIMS) by the National Bureau of Standards. The ${ }^{187} \operatorname{Re} /{ }^{185} \operatorname{Re}$ of $1.6740 \pm 0.0011$ was obtained from 66 individual analyses calibrated against 63 analyses of seven gravimetrically prepared calibration standards. PTIMS ionizes a sample though direct heating on a metal filament; it is therefore limited in materials that can be effectively ionized to those with relatively low first ionization potential. The first ionization potential of $\mathrm{Re}-7.86404(10) \mathrm{eV}$ (CAMPBELLMiller AND Simard, 1996) — is high relative to elements routinely analyzed by PTIMS. Though beam intensity data needed to calculate the ionization efficiency were not provided in the 1973 study, the relative difficulty in ionizing Re may be 
inferred from Figure 3-1, and from the total amount of Re loaded onto the filaments

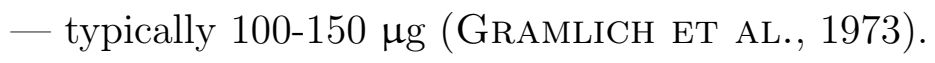

Rhenium ionization efficiency was significantly improved by the development of negative thermal ionization mass spectrometry (NTIMS), in which Re is ionized as an anionic (principally $\mathrm{ReO}_{4}^{-}$) as opposed to cationic $\left(\mathrm{Re}^{+}\right.$) species (CREASER ET AL., 1991). This improvement allows for routine analysis of a single ng of Re -5 orders of magnitude less analyte than used in the PTIMS study (CREASER ET AL., 1991). Initial within-run uncertainties on ${ }^{187} \operatorname{Re} /{ }^{185}$ Re measured by NTIMS ( 330 ppm), are essentially equivalent to the mass spectrometric uncertainty cited by GRAMLICH ET AL. (1973, 270 ppm), though uncertainties in later studies of the $\mathrm{W}$ system were significantly lower (e.g. HARPER AND JACOBSEN, 1996, 10 ppm). Within-run uncertainties for modern analyses of Re are rarely reported. Using NTIMS, a second absolute Re isotope abundance determination was done by total evaporation yielding a ${ }^{187} \operatorname{Re} /{ }^{185} \operatorname{Re}$ of $1.6755 \pm 0.0014$ (SUzUKI ET AL., 2004). This is within uncertainty of the original SRM 989 value of $1.6740 \pm 0.0011$ (GRAMLICH ET AL., 1973), though it must be noted that the NTIMS study did not use SRM 989 for its abundance determination.

While conventional thermal ionization is ultimately limited by the melting temperature of the filament material, a radio frequency (rf) induced Ar plasma source can reach up to $7500 \mathrm{~K}$, ionizing even those elements with very high first ionization potentials (Houk ET AL., 1980; Houk, 1986). Coupling of the plasma source with a double-focusing magnetic sector mass analyzer and a movable multi-collector array led to the development of multi-collector inductively-coupled plasma mass spectrometry, or MC-ICPMS (Walder ANd Freedman, 1992; Walder et AL., 1993; HalliDAY ET AL., 1995). The mass analyzer produces the "flat-topped peaks" required for precise analyses while the multi-collector array allows simultaneous acquisition of multiple ion beams, accounting for the rapid variations in signal strength inherent to a plasma source (WALDER AND FreEdman, 1992; HALliday ET AL., 1995). 

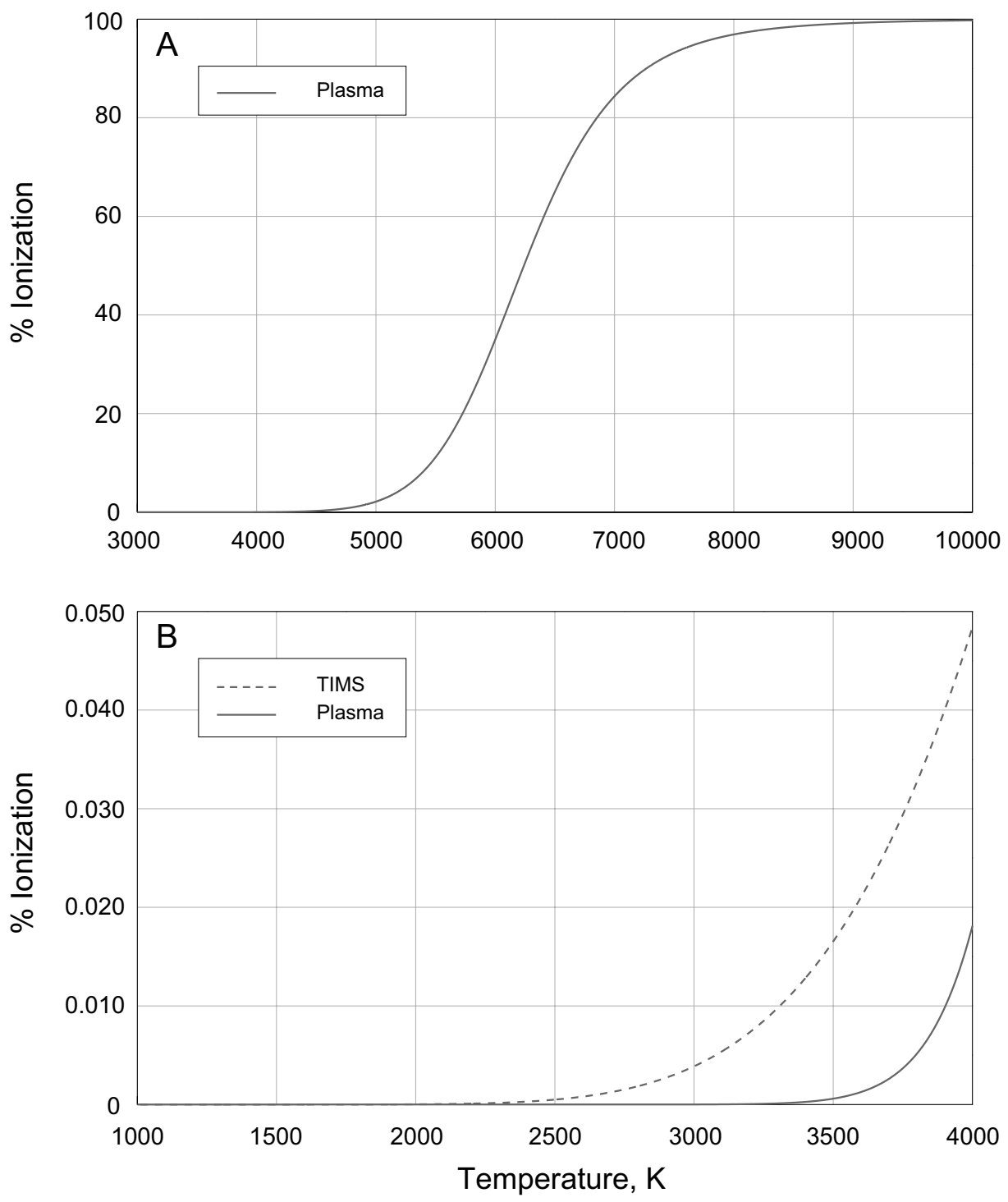

Figure 3-1: Re ionization efficiency vs. temperature for PTIMS and plasma ion sources. Thermal (PTIMS) ionization is estimated with the Saha-Langmuir equation (Dresser, 1968) and a Re work function of $5.15 \mathrm{eV}$ (KAWANo, 2006; DĄBEK And Stanislaw, 2007). Plasma ionization is estimated with the Saha equation Boumans (1968) and an electron density of $10^{15} \mathrm{~cm}^{-3}$ (Houk, 1986; Miller, 1992). Both use electronic partition function estimates from a fifth-order polynomial (DE GALAN ET AL., 1968).

A: Estimated Re plasma ionization from 3000-10000K. Examination of PTIMS ionization is not appropriate for these temperatures.

B: Magnified view of 1000-4000K. A very low degree of ionization $(\sim 0.015 \%)$ is achieved at the Re melting temperature of $3450 \mathrm{~K}$; the ionization estimate corresponding to the analytical temperature of $2100^{\circ} \mathrm{C}$ (GRAMLICH ET AL., 1973) is $0.00023 \%$. 
Thermal ionization mass spectrometry (PTIMS, NTIMS) can exhibit significant within-run isotopic variation due to the successive sampling (ionization) of a closed (finite) sample load (Russel ET AL., 1978; HART AND ZINDleR, 1989); that is, sample analyses show time-dependent fractionation of the entire sample reservoir. In contrast, MC-ICPMS ionizes a sample solution that is continuously aspirated from a homogenous source meaning the sample reservoir remains unfractionated during the course of an analysis.

The main advantage of MC-ICPMS is that it provides isotope ratio measurements of elements with high ionization potential at sufficient precision to resolve differences at the sub-\%o level. Because the ion source remains "on," and samples are often introduced to the source as solutions, MC-ICPMS lends itself to automation and increased sample throughput; it has resulted in the recent exploration of so-called "non-traditional" stable isotope systems; Cr, Fe, Cu, Zn, Mo, Cd, W, Hg, and Tl are examples (LeE AND Halliday, 1995a,b; BeArd et Al., 1999; MarÉchal ET AL., 1999; Ellis et AL., 2002; Arnold Et AL., 2004; Nielsen ET AL., 2004; BergQuist And Blum, 2007; Ripperger AND REHKäMPER, 2007). This study is the first investigation of stable isotope fractionation of Re.

\subsection{Analytical methods}

\subsubsection{Materials}

Data are presented for commercially available and in-house standards, as well as for one natural reference material. Commercial standards include the isotopically certified SRM 989 (GRAMLICH ET AL., 1973), as well as certified Re concentration standards NIST SRM 3143 and Alfa Aesar Specpure ${ }^{\circledR}$ (Lot \#235760A). In-house reference materials were created from perrhenic acid (HReO4) and a zone-refined Re ribbon from H. Cross Co. The natural reference material is the Devonian Ohio Shale standard USGS SDO-1 (KANE ET AL., 1990). 

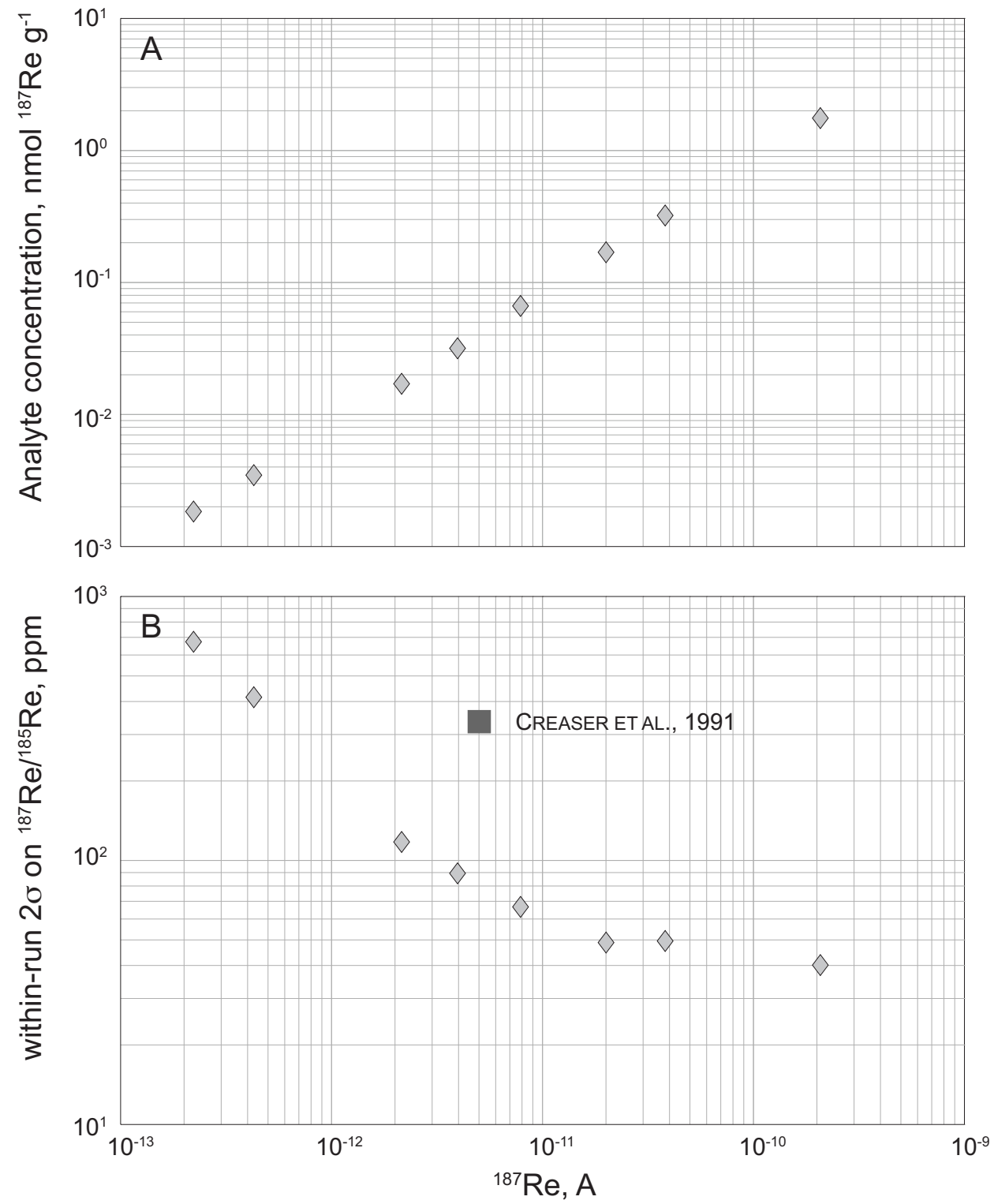

Figure 3-2: A: Plot of the linear relationship between ion beam intensity (in amps, A) and ${ }^{187}$ Re concentration.

B: Plot of the within-run uncertainty on ${ }^{187} \mathrm{Re} /{ }^{185} \mathrm{Re}$ for the same solutions. Published NTIMS data are also plotted. (CREASER ET AL., 1991). For equivalent ion beam intensities, within-run uncertainties for MC-ICPMS analyses are markedly lower than those CREASER ET AL. (1991). 


\subsubsection{Sample processing}

Several grams of well-mixed USGS SDO-1 (Devonian Ohio Shale) sample powder were ashed overnight at $550^{\circ} \mathrm{C}$ before being subjected to a series of strong acid leaches (concentrated $\mathrm{HCl}$, aqua regia, concentrated $\mathrm{HNO}_{3}$ ). Though SDO-1 is not certified for Re concentration, in-house tests show that this leaching liberates $>85 \%$ of total Re. Leached Re was purified by column chromatography using AG1X8 anion resin (100-200 mesh). Sample material in $0.5 \mathrm{~mol} \mathrm{~L}^{-1} \mathrm{HNO}_{3}$ was loaded onto $1 \mathrm{~mL}$ of resin, rinsed with $15 \mathrm{~mL}$ of $0.5 \mathrm{molL}^{-1} \mathrm{HNO}_{3}$, before elution with $12 \mathrm{~mL}$ of $4 \mathrm{~mol}$ $\mathrm{L}^{-1} \mathrm{HNO}_{3}$. Chromatographic purification of Re yields a sample matrix with some elements present in much higher abundances than Re. The effects of chromatographic separation and sample matrix on isotope ratio determinations are discussed later (see Sections 3.5.2 and 3.5.3).

\subsubsection{Mass spectrometry}

All isotope analyses are done with a Thermo Fisher Scientific NEPTUNE. The Faraday cup configuration ${ }^{180} \mathrm{~W}$ (L3), ${ }^{182} \mathrm{~W}$ (L2), ${ }^{183} \mathrm{~W}$ (L1), ${ }^{184} \mathrm{~W}$ (Axial), ${ }^{185} \mathrm{Re}$ (H1), ${ }^{186} \mathrm{~W}(\mathrm{H} 2),{ }^{187} \mathrm{Re}(\mathrm{H} 3)$, and ${ }^{189} \mathrm{Os}(\mathrm{H} 4)$ is used to measure ion beams in static mode with $10^{11} \Omega$ resistors. Because of the potential for Os interferences on ${ }^{184} \mathrm{~W},{ }^{186} \mathrm{~W}$, and especially ${ }^{187} \mathrm{Re}$ due to naturally variable ${ }^{187} \mathrm{Os}$, the axial secondary electron multiplier (SEM) is also used to monitor ${ }^{189} \mathrm{Os}$. Samples dissolved in $1 \mathrm{~mL}$ of 0.5 mol L ${ }^{-1} \mathrm{HNO}_{3}$ are introduced using a PFA MicroFlow nebulizer (Elemental Scientific Incorporated), a quartz spray chamber, and X-cones. Samples are ionized using an radio frequency generator power of $1170 \mathrm{~W}$. Ion beams are integrated for 25 cycles of $16.77 \mathrm{~s}$ for a total acquisition time of $\sim 420 \mathrm{~s}$. Approximately $500 \mu \mathrm{L}^{-1}$ are consumed. Analyte concentrations are either $20 \mathrm{ng} \operatorname{Re~g}^{-1}$ and $70 \mathrm{ng} \mathrm{W} \mathrm{g}{ }^{-1}$, or $10 \mathrm{ng} \mathrm{Re} \mathrm{g}^{-1}$ and $35 \mathrm{ng} \mathrm{W} \mathrm{g}^{-1}$. Atomic transmission is about $1 \times 10^{-5}$. The inlet system is washed with $0.5 \mathrm{molL}^{-1} \mathrm{HNO}_{3}$ for 3 minutes between samples. 


\subsection{Correction of instrumental mass fractionation}

Use of an MC-ICPMS (or any mass spectrometer) for isotope analysis introduces an instrumental mass fractionation (also called the mass bias or instrumental mass bias). In MC-ICPMS, instrumental fractionation from true isotopic values is caused by preferential transmission of more massive isotopes into the mass spectrometer; this is due to space-charge effects in the plasma and gaseous expansion between the sample and skimmer cones (WALDER AND FrEEDMAN, 1992; WALDER ET AL., 1993). Because the introduced fractionation can be several percent, it is crucial that it be properly characterized and corrected for in order to characterize the permillevel natural isotopic fractionation typically seen in transition metal isotope systems. All forms of instrumental mass bias correction involve the application of some factor applied to the measured isotopic ratio, in this case:

$$
\frac{{ }^{187} R e}{{ }^{185} \operatorname{Re}} \operatorname{corr}=\frac{{ }^{187} \text { Re }}{{ }^{185} \text { Re }} \text { meas } \times \text { Factor }
$$

There are several ways of determining the correcting factor; samples can be doublespiked, standard-bracketed, or externally-corrected. Double-spiking is the addition of two isotopically enriched spikes and is particularly advantageous because it allows simultaneous determination of analyte concentration, and explicit instrumental mass bias correction for the element of interest (Russel ET AL., 1978; GALER, 1999). However, it requires a minimum of four isotopes. As Re has only two naturally

occurring isotopes and no "artificial" or "synthetic" nuclides that are sufficiently long-lived to serve as isotopic spikes, we are left with a choice or some combination of standard-bracketing correction and external correction.

\subsubsection{Standard-bracketing correction}

Standard-bracketing correction is done by analyzing standards before and after every sample. For each standard, the instrumental mass bias is determined by comparing 
measured and accepted ${ }^{187} \mathrm{Re} /{ }^{185} \mathrm{Re}$ values. These factors are then averaged and applied to the intervening sample. Averaging the standard factors is effectively a linear interpolation of the changing instrumental mass bias. Mathematically, the factor can be expressed as:

$$
\text { Factor }=\frac{\frac{187 R e}{185 R e} S R M 989_{\text {meas }, 1}+\frac{187 R e}{185} \text { Re } R M 989_{\text {meas }, 2}}{2 \times \frac{187 R e}{185 R e} S M M 89_{\text {true }}}
$$

The advantages provided by this method result from its simplicity. Not only is standard-bracketing conceptually and mathematically simple, but by incorporating the accepted value of the Re standard, the correction forces the measured standard ${ }^{187} \mathrm{Re} /{ }^{185} \mathrm{Re}$ to the true value ensuring that data will be expressed relative to $0 \% 0$ regardless of any differences between corrected standard values and the accepted values (see also section 3.3.2). However, because the correction factors are generated from data acquired before and after the sample data, standard-bracketing does not

represent "real time" correction. Because plasma sources undergo rapid changes in ionization, standard-bracketing is temporally coarse; transient changes in ionization of the bracketing standard(s) may result in a correction factor inappropriate to the sample, while such changes occurring during sample analysis may not be accounted for by the correction factor.

\subsubsection{External correction}

In contrast, external or inter-element correction provides a correction factor representative of the real-time instrumental mass bias. This is achieved by simultaneous data acquisition for both the element of interest, in this case Re, and some artificially added ("doped") element of known isotopic composition. The instrumental mass fractionation is assumed to be identical for both elements. Common examples are the use of $\mathrm{Cu}$ to correct $\mathrm{Zn}$ and vice-versa as well as the use of $\mathrm{Tl}$ to correct Pb (Longerich et AL., 1987; Walder et Al., 1993; MarÉchal Et Al., 1999; 
White et Al., 2000; Collerson et Al., 2002).

\section{Choice of doping element}

Both Re and the doped element must be measured simultaneously. The ThermoFinnigan Neptune mass dispersion range of $\sim 17 \%$ allows a choice of doping elements in the mass range of $156\left({ }^{187} \mathrm{Re}\right.$ in cup H4) to $216\left({ }^{185} \mathrm{Re}\right.$ in cup L4). Eliminating monoisotopic elements (e.g. Au) and those composed of nuclides with very short half-lives (e.g. Po) we are left with Gd, Dy, Er, Yb, Lu, Hf, Ta, W, Ir, Pt, Hg, Tl, Pb. Osmium is not included as ${ }^{187} \mathrm{Os}$ is an isobar of ${ }^{187} \mathrm{Re}$. Because instrumental mass fractionation is mass dependent (WALder and Freedman, 1992; Walder et AL., 1993; HiRATA, 1996), elements with average masses close to that of Re are more desirable. On this basis, we have chosen $\mathrm{W}$ whose average mass of $\sim 184 \mathrm{amu}$ is very close to the average Re mass of $\sim 186 \mathrm{amu}$; a converse study, in which Re is used to correct W, has already been done (IRISAWA AND Hirata, 2006). In addition, W and Re have similar first ionization potentials, $7.86404(10) \mathrm{eV}$ and $7.83352(11) \mathrm{eV}$ respectively (CAmpbell-Miller and Simard, 1996), suggesting that they should ionize to a similar degree in the plasma source (Houk, 1986).

This study uses a commercially available W concentration standard, NIST SRM 3163, to correct Re data. Though numerous studies have employed SRM 3163 as an isotopic standard (Lee and Halliday, 1995a; Irisawa And Hirata, 2006; MARKOWSKI ET AL., 2006), it has never been isotopically characterized in an absolute sense (gravimetrically); all studies reporting data for SRM 3163 do so accepting values originally reported in the relative isotopic study of a Merck $\mathrm{Na}_{2} \mathrm{WO}_{4}$ standard (VÖLKENING ET AL., 1991a).

\section{Choice of correcting ratio}

Tungsten is composed of five isotopes with the following atomic abundances $-{ }^{180} \mathrm{~W}$ at $0.0012(1),{ }^{182} \mathrm{~W}$ at $0.2650(16),{ }^{183} \mathrm{~W}$ at $0.1431(4),{ }^{184} \mathrm{~W}$ at $0.3064(2)$, and ${ }^{186} \mathrm{~W}$ 
at 0.2843(19) (VÖlkening ET Al., 1991a; Rosman And TAYlor, 1998), providing us with a variety of ratios $-{ }^{186} \mathrm{~W} /{ }^{184} \mathrm{~W},{ }^{186} \mathrm{~W} /{ }^{183} \mathrm{~W},{ }^{186} \mathrm{~W} /{ }^{182} \mathrm{~W},{ }^{184} \mathrm{~W} /{ }^{183} \mathrm{~W}$, ${ }^{184} \mathrm{~W} /{ }^{182} \mathrm{~W}$, and ${ }^{183} \mathrm{~W} /{ }^{182} \mathrm{~W}$ - with which to correct our measured ${ }^{187} \mathrm{Re} /{ }^{185} \mathrm{Re}$. Ratios incorporating ${ }^{180} \mathrm{~W}$ are not considered due to the lesser degree of precision at which they can be measured due to the hundredth-fold abundance of ${ }^{180} \mathrm{~W}$ relative to other isotopes of $\mathrm{W}$.

A previous study of the instrumental mass fractionation of $\mathrm{Nd}$ noted greater accuracy (relative to an accepted PTIMS value) with greater similarity in the "ratio mass" (the average of the numerator and denominator masses) of the correcting and corrected ratios (VANCE AND THIRLWALL, 2002). In essence, this is an intra-elemental extension of the mass dependence of instrumental mass fractionation previously noted at the inter-elemental level (WALDER AND FREEDMAN, 1992; WALDER ET AL., 1993; HirATA, 1996). On this basis, this study uses ${ }^{186} \mathrm{~W} /{ }^{184} \mathrm{~W}$ (ratio mass of 184.95265 $\mathrm{amu}$ ) to correct ${ }^{187} \mathrm{Re} /{ }^{185} \mathrm{Re}$ (ratio mass of $185.95435 \mathrm{amu}$ ). Correcting ratios with ratio masses that are even closer to that of ${ }^{187} \mathrm{Re} /{ }^{185} \mathrm{Re}$ exist only in the Os system (e.g. ${ }^{188} \mathrm{Os} /{ }^{184} \mathrm{Os}$, ratio mass 185.95416$)$, but are not considered due to the presence of isobaric ${ }^{187} \mathrm{Os}$.

Furthermore, analysis of standard data over the course of this study indicates that ${ }^{186} \mathrm{~W} /{ }^{184} \mathrm{~W}$ is measured with the highest within-run precision of all $\mathrm{W}$ isotope ratios.

\section{Choice of fractionation law}

Though Rayleigh diffusion and Langmuir ionization equations provide a theoretical framework for mass fractionation in a TIMS instrument (HABFAST, 1998), MCICPMS uses one of several empirically-defined fractionation "laws" to describe instrumental mass bias. Calculation of the correction factor depends on the law used; most common are the linear, power, exponential, and Rayleigh (RAYLEIGH, 1902) laws. The correction factors for these laws, adapted for correction of ${ }^{187} \operatorname{Re} /{ }^{185} \mathrm{Re}$ with ${ }^{186} \mathrm{~W} /{ }^{184} \mathrm{~W}$ are expressed as follows (after HART AND ZindLER, 1989). 
Linear law:

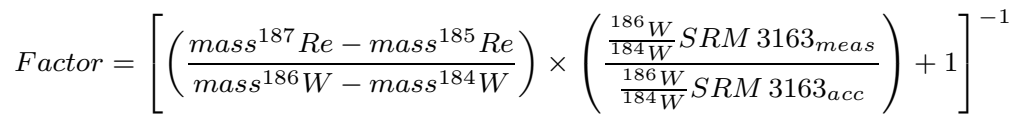

Power law:

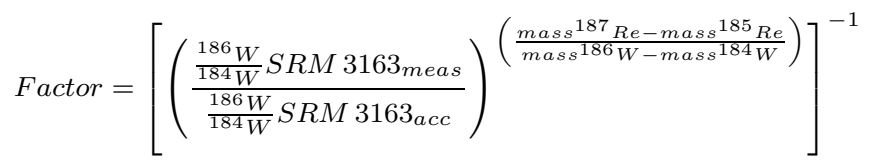

Exponential law:

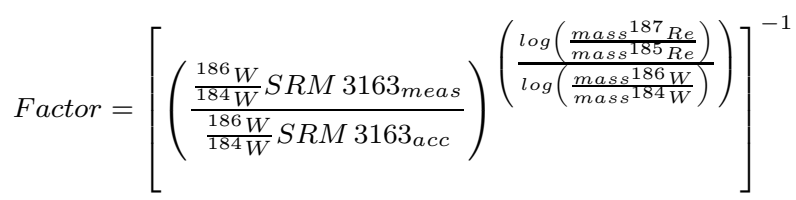

Rayleigh law:

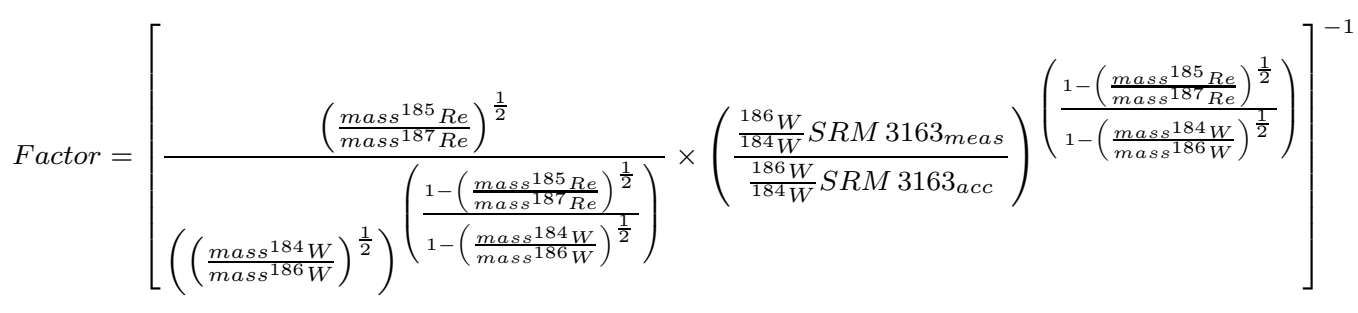

Figure 3-3 is a conceptual illustration of the effects of instrumental mass bias on a mixed solution of SRM 989 and SRM 3163 for ${ }^{187} \mathrm{Re} /{ }^{185} \mathrm{Re}$ and ${ }^{186} \mathrm{~W} /{ }^{184} \mathrm{~W}$ in $\log _{e}$ space.

Fractionation trends corresponding to linear, power, Rayleigh, and exponential laws are plotted. Also shown is a magnification of the region corresponding to $\sim 0.75 \%$ $\mathrm{amu}^{-1}$, which is representative of the degree of mass fractionation experienced by real samples (see Figure 3-4). Choice of the linear or power laws yield essentially identical results for the corrected ratio; the same is true of the Rayleigh or exponential laws. However, choice between these two sets of laws can affect the values of corrected ratios by $\sim 80 \mathrm{ppm}$. 

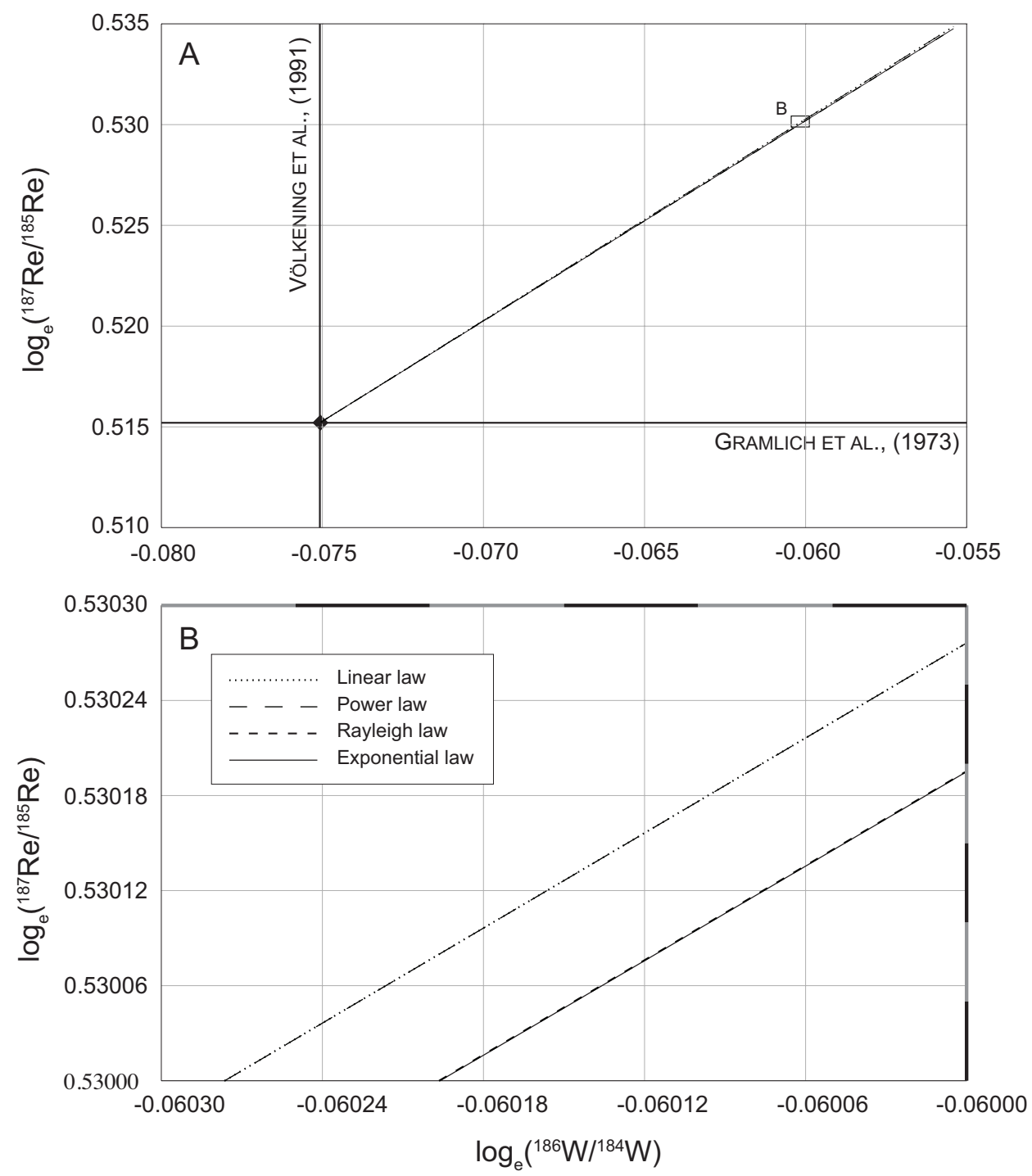

Figure 3-3: Theoretical plot of instrumental mass fractionation of a mixed SRM 989 Re and SRM 3163W solution in $\log _{e}$ space.

A: ${ }^{187} \mathrm{Re} /{ }^{185}$ Re vs. ${ }^{186} \mathrm{~W} /{ }^{184} \mathrm{~W}$. Standard values are indicated (GRAMLICH ET AL., 1973; VÖLKEning ET AL., 1991a). Trends of linear, power, exponential, and Rayleigh fractionation from assumed standard values are plotted.

B: Magnification around area corresponding to $\sim 0.75 \% \mathrm{amu}^{-1}$ mass fractionation. Scale bars at top and right are $50 \mathrm{ppm}$ of measured values. Note that this figure also graphically represents W-correction. Measured values of $\log _{e}\left({ }^{187} \mathrm{Re} /{ }^{185} \mathrm{Re}\right)$ are projected back to the accepted value of $\log _{e}\left({ }^{186} \mathrm{~W} /{ }^{184} \mathrm{~W}\right)$ according to some fractionation law (slope); the corrected value of $\log _{e}\left({ }^{187} \mathrm{Re} /{ }^{185} \mathrm{Re}\right)$ is then exponentiated to give the corrected ${ }^{187} \mathrm{Re} /{ }^{185} \mathrm{Re}$. 
The extent to which the the choice of fractionation law affects the value of corrected ratios is related to the choice of correcting ratio first discussed in section 3.3.2. Likewise the choice of fractionation law has the smallest effect in situations where the ratio masses of corrected and correcting ratios are the most similar. For example, while the choice of fractionation law can result in a corrected value spread of up to $\sim 80 \mathrm{ppm}$ when correcting ${ }^{187} \mathrm{Re} /{ }^{185} \mathrm{Re}$ with ${ }^{186} \mathrm{~W} /{ }^{184} \mathrm{~W}$ (a ratio mass difference of $1.00171 \mathrm{amu}$ ), use of ${ }^{186} \mathrm{~W} /{ }^{183} \mathrm{~W}$ to correct ${ }^{187} \mathrm{Re} /{ }^{185} \mathrm{Re}$ (a ratio mass difference of $1.50216 \mathrm{amu}$ ) results in a spread of $\sim 250 \mathrm{ppm}$.

PTIMS-based studies of instrumental mass bias indicate that the exponential law is the most accurate way to correct instrumental mass bias (RUSSEL ET AL., 1978; HART AND ZINDLER, 1989). MC-ICPMS studies indicate that either the power law (Taylor ET Al., 1995; Hirata, 1996; VAnce And Thirlwall, 2002), the exponential law (TAYlor ET AL., 1995; LuAis Et AL., 1997; MARÉCHAL ET AL., 1999; White ET AL., 2000), or modified versions thereof (HiRATA, 1996; MARÉCHAL ET AL., 1999; White ET AL., 2000; IrIsawa AND Hirata, 2006) provide the best fit to observed data.

Figure 3-4 is a plot of 281 analyses of solution mixtures of SRM 989 and SRM 3163 in $\log _{e}$ space. The data define an array of slope 1.0065 (40). Note also that the data do not trend through the intersection of accepted values of SRM 989 and SRM 3163 (GRAMlich ET AL., 1973; VÖLKENING ET AL., 1991b); see also section 3.3.2. Trends of raw ${ }^{187} \mathrm{Re} /{ }^{185} \mathrm{Re}$ and various correcting ratios of $\mathrm{W}$ are listed in Table 3.1; also included are trends calculated using the linear, power, Rayleigh, and exponential empirical fractionation laws. Though the observed fractionation trend (slope) is consistent with those of the linear and power laws for our chosen correcting ratio, ${ }^{186} \mathrm{~W} /{ }^{184} \mathrm{~W}$, this consistency is not seen across all choices of correcting ratio. Likewise, none of the empirical laws can be chosen on the basis of the similarity between observed and predicted fractionation trends. Other studies observing this have ascribed it to differences in fractionation between corrected and correcting elements 

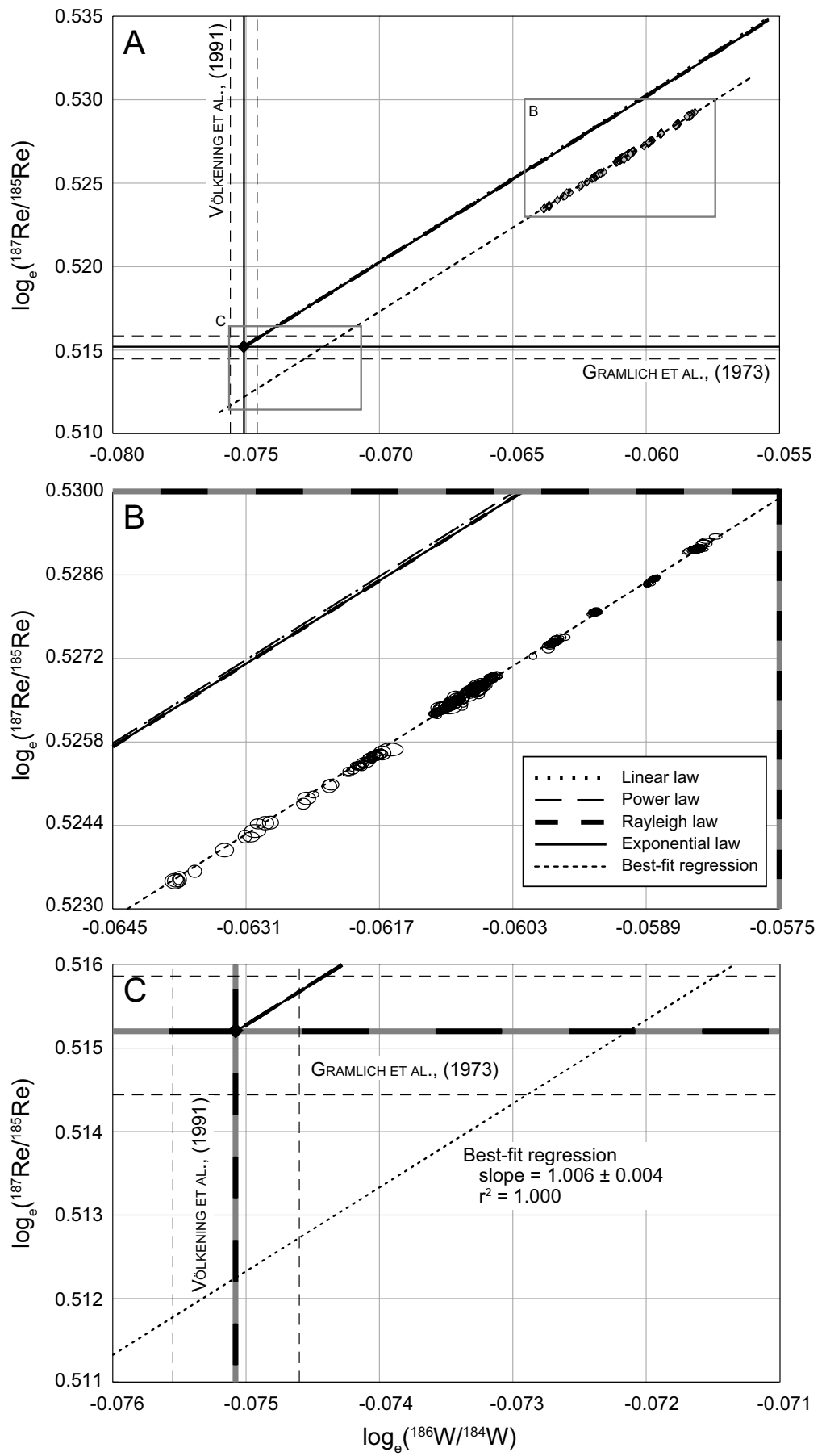

Figure 3-4: Full caption on page 140. 
Figure 3-4: ${ }^{187} \mathrm{Re} /{ }^{185} \mathrm{Re}$ and ${ }^{186} \mathrm{~W} /{ }^{184} \mathrm{~W}$ for 283 analyses mixed SRM 989 and SRM 3163 solutions in $\log _{e}$ space. Also plotted are the accepted values for SRM 989 and SRM 3163, fractionation trends for the four empirical fractionation laws, and the observed fractionation trend - obtained by type II linear regression.

A: ${ }^{187} \operatorname{Re} /{ }^{185} \mathrm{Re}$ and ${ }^{186} \mathrm{~W} /{ }^{184} \mathrm{~W}$ in the context of the accepted isotopic values of SRM 989 and SRM 3163. Also shown are uncertainty envelopes (2 s.d.) for accepted values of SRM 989 and SRM 3163 (Gramlich ET AL., 1973; VÖLKEning ET AL., 1991a), as well as linear law, power law, exponential law and Rayleigh law fractionation trends, along with the best-fit type II regression line.

B: A magnification of the area corresponding to an instrumental mass bias of $\sim 0.75 \%$ $\mathrm{amu}^{-1}$. Scale bars at top and right are $0.5 \%$. Data from individual analytical sessions show up as clusters. Data from individual sessions also show offsets to best-fit regression line; in many cases these offsets are produced by differing radio frequency power (rf) and can result in $0.1 \%$-level differences in $\mathrm{W}$-corrected $\delta^{187} \mathrm{Re}$ (see also Table 3.2).

C: A magnification of the area of the accepted isotopic values of SRM 989 and SRM 3163 (Gramlich et Al., 1973; Völkening et AL., 1991a). Scale bars are 0.5\%o. The intersection of the observed fractionation trend and the accepted values for SRM 989 and SRM 3163 gives corrected values well outside the quoted uncertainties; see text for further details.

(MarÉchal et Al., 1999; Hirata, 1996). Also, when observing figure 3-4, raw data from different analytical sessions appear somewhat offset from one another relative to the best-fit regression; this might also result from small differences in elemental ionization or instrumental mass bias between sessions. In some cases, these differences result from differing rf power for individual analytical sessions occurring on the same day, Table 3.2).

Deviations from expected fractionation based on elemental differences should disappear when examining a single element system. Figure 3-9 is a series of W threeisotope plots in $\log _{e}$ space, and Table 3.4 shows fractionation trends (type II regression slopes) for all possible $\mathrm{W}$ isotope ratios (that have the more massive nuclide as the numerator and excepting those incorporating ${ }^{180} \mathrm{~W}$ ) as well as fractionation trends predicted by the empirical fractionation laws. As with $\log _{e}{ }^{187} \operatorname{Re} /{ }^{185} \operatorname{Re} \mathrm{vs} \log e$ ${ }^{186} \mathrm{~W} /{ }^{184} \mathrm{~W}$, individual regressions might be consistent with one or more fractionation laws, but this does not extend across the range of correcting and corrected ratio choices. 


\begin{tabular}{rcccccc}
\hline Correcting ratio & ${ }^{186} \mathrm{~W} /{ }^{184} \mathrm{~W}$ & ${ }^{186} \mathrm{~W} /{ }^{183} \mathrm{~W}$ & ${ }^{186} \mathrm{~W} /{ }^{182} \mathrm{~W}$ & ${ }^{184} \mathrm{~W} /{ }^{183} \mathrm{~W}$ & ${ }^{184} \mathrm{~W} /{ }^{182} \mathrm{~W}$ & ${ }^{183} \mathrm{~W} /{ }^{182} \mathrm{~W}$ \\
\hline Observed slope & $1.0065(40)$ & $0.6702(39)$ & $0.4992(24)$ & $2.0244(216)$ & $0.9947(57)$ & $1.9514(119)$ \\
& & & & & & \\
Linear law slope & $\mathbf{0 . 9 9 9 7}$ & $\mathbf{0 . 6 7 1 6}$ & 0.5074 & 1.9865 & $\mathbf{1 . 0 0 0 0}$ & 1.9838 \\
Power law slope & $\mathbf{0 . 9 9 9 7}$ & $\mathbf{0 . 6 6 6 7}$ & $\mathbf{0 . 4 9 9 9}$ & 2.0014 & $\mathbf{1 . 0 0 0 0}$ & 1.9988 \\
Rayleigh law slope & 0.9943 & 0.6622 & 0.4959 & 1.9718 & 0.9839 & $\mathbf{1 . 9 5 8 5}$ \\
Exponential law slope & 0.9943 & 0.6613 & 0.4945 & 1.9744 & 0.9839 & $\mathbf{1 . 9 6 1 1}$ \\
\hline
\end{tabular}

Table 3.1: List of observed (regression) and theoretically calculated (linear, power, Rayleigh, and exponential, law) factors (slopes) describing instrumental mass bias relationship between ${ }^{187} \mathrm{Re} /{ }^{185} \mathrm{Re}$ and various correcting ratios of $\mathrm{W}$ in $\log _{e}$ space. Included with the best-fit regression slopes are 2 s.d. uncertainties. Theoretical values of calculated power, Rayleigh, and exponential slopes are equivalent to the exponential term in the denominator of the factors corresponding to these laws; their uncertainties are governed by the uncertainties in nuclide masses which are sub ppm and can therefore be ignored. Though a linear fractionation trend is not a straight line in $\log _{e}$ space, its degree of curvature is very small; the "slope" of the linear trend has been approximated by taking the ratio of the change in $\log _{e}{ }^{187} \mathrm{Re} /{ }^{185} \mathrm{Re}$ (calculated) to the change in $\log _{e}{ }^{18 X} \mathrm{~W} /{ }^{18 Y} \mathrm{~W}$ (observed). Factors shown in bold-face type are within the uncertainty of the best-fit linear regression. 
Consideration of actual data can, therefore, not be used to select the "best" fractionation law for either the correction of ${ }^{187} \mathrm{Re} /{ }^{185} \mathrm{Re}$ with $\mathrm{W}$, or the correction of $\mathrm{W}$ with $\mathrm{W}$. The failure of the empirical fractionation laws to account for intra-elemental isotope fractionation has been implicitly and explicitly noted for $\mathrm{Zn}, \mathrm{Nd}$, and $\mathrm{Pb}$ in previous studies (HIRATA, 1996; MARÉCHAl ET AL., 1999; VANCE AND ThIRLWALl, 2002). Specifics of $W$ and Re fractionation presented in this study — both the average and especially the range of mass bias - are smaller than those seen for $\mathrm{Cu}, \mathrm{Zn}$, and Nd (Maréchal et Al., 1999; Vance And Thirlwall, 2002). This is expected due to the greater mass of the Re system, but still noteworthy because the smaller range in mass bias results in larger uncertainty for the regressed slopes (fractionation factors), and the overlap with expected empirical fractionation factors is more likely for Re than for any other isotopic system considered thus far.

As previously noted, TIMS exhibits a much larger range of isotopic fractionation over the course of a long sample run, and TIMS studies indicate that the exponential law best (though not fully) describes instrumental mass fractionation (RUSSEL ET AL., 1978; HART AND Zindler, 1989). As a result, this study will employ the exponential law for the correction of ${ }^{187} \mathrm{Re} /{ }^{185} \mathrm{Re}$ with ${ }^{186} \mathrm{~W} /{ }^{184} \mathrm{~W}$. Examination of the intersection of the best-fit fractionation factor with accepted values for ${ }^{186} \mathrm{~W} /{ }^{184} \mathrm{~W}$, ${ }^{183} \mathrm{~W} /{ }^{184} \mathrm{~W}$, and ${ }^{182} \mathrm{~W} /{ }^{184} \mathrm{~W}$ in Figure 3-9 indicates that the correction of $\mathrm{W}$ isotopic values gives values identical within uncertainty to those determined using NTIMS; therefore application of the empirical fractionation laws do not yield significantly different results based on ion source. 


\section{Accuracy and precision of $\mathrm{W}$-corrected ${ }^{187} \mathrm{Re} /{ }^{185} \mathrm{Re}$ : towards a combined standard-bracketing-W-correction}

Rhenium and $\mathrm{W}$ isotope fractionation during MC-ICPMS analysis are highly correlated, and the use of different $\mathrm{W}$ ratios or fractionation laws affects the corrected $\delta^{187} \mathrm{Re}$ and $\delta^{186} \mathrm{~W} /{ }^{184} \mathrm{~W}$ only to very minor degrees. Despite this, ${ }^{186} \mathrm{~W} /{ }^{184} \mathrm{~W}$ correction of ${ }^{187} \operatorname{Re} /{ }^{185} \operatorname{Re}$ for SRM yields $\delta^{187} \operatorname{Re}=-2.8 \%$, a value well outside the quoted 2 s.d. uncertainty of $0.66 \%$ (GRAMLICH ET AL., 1973); likewise, Re-correction of ${ }^{186} \mathrm{~W} /{ }^{184} \mathrm{~W}$ yields a ${ }^{186} \mathrm{~W} /{ }^{184} \mathrm{~W}$ value of $+2.8 \%$, a value also outside the quoted 2 s.d. uncertainty of $0.25 \%$ (VÖLKEnING ET AL., 1991a); see Figure 3-4. This discrepancy can be explained by an incorrect value of ${ }^{187} \mathrm{Re} /{ }^{185} \mathrm{Re}$ for $\mathrm{SRM} 989$, an incorrect value of ${ }^{186} \mathrm{~W} /{ }^{184} \mathrm{~W}$ for SRM 989, or some combination thereof. Given that ${ }^{187} \mathrm{Re} /{ }^{185} \mathrm{Re}$ was absolutely determined (gravimetrically, GRAMLICH ET AL., 1973), while ${ }^{186} \mathrm{~W} /{ }^{184} \mathrm{~W}$ was only relatively determined (internally normalized, VöLKENING ET AL., 1991a), it is likely that that the principal fault lies with the accepted value of ${ }^{186} \mathrm{~W} /{ }^{184} \mathrm{~W}$ used in the $\mathrm{W}$-correction factor. We are, therefore, faced with a dilemma; while increasing the value of ${ }^{186} \mathrm{~W} /{ }^{184} \mathrm{~W}$ used in our correction factor by $+2.8 \%$ o would bring our corrected $\delta^{187} \mathrm{Re}$ in line with the absolutely-determined ${ }^{187} \mathrm{Re} /{ }^{185} \mathrm{Re}$, many other studies have published data using the relative isotopic composition of $\mathrm{W}$ (e.g. Lee And Halliday, 1995a; Irisawa And Hirata, 2006; Markowski et AL., $2006)$; conversely, use of established though relative published values of ${ }^{186} \mathrm{~W} /{ }^{184} \mathrm{~W}$ in the $\mathrm{W}$-correction factor results in a several $\%$ deviation of $\delta^{187}$ Re from its absolutely determined value.

Values and uncertainties of $\delta^{187}$ Re for 161 analyses of mixed solutions of SRM 989 and SRM 3163 across eight analytical sessions are listed in Table 3.2. Within an analytical session, $\mathrm{W}$-correction is, in general, as precise or more precise than standard-sample bracketing; this is especially true for ${ }^{186} \mathrm{~W} /{ }^{184} \mathrm{~W},{ }^{186} \mathrm{~W} /{ }^{183} \mathrm{~W}$, and ${ }^{186} \mathrm{~W} /{ }^{182} \mathrm{~W}$ correcting ratios. However, in addition to the substantial perturbation of $\mathrm{W}$-corrected data already noted, there also appears to be significant variability 


\begin{tabular}{|c|c|c|c|c|c|c|c|c|c|c|c|c|c|c|c|c|c|c|}
\hline & \multicolumn{2}{|c|}{$\begin{array}{l}7 / 20 / 2007 \\
1 \mathrm{st}, n=47\end{array}$} & \multicolumn{2}{|c|}{$\begin{array}{c}7 / 20 / 2007 \\
2 \text { nd, } n=47\end{array}$} & \multicolumn{2}{|c|}{$\begin{array}{c}7 / 20 / 2007 \\
3 \mathrm{rd}, n=47\end{array}$} & \multicolumn{2}{|c|}{$\begin{array}{c}9 / 08 / 2007 \\
1045 \mathrm{~W}, n=24\end{array}$} & \multicolumn{2}{|c|}{$\begin{array}{c}9 / 08 / 2007 \\
1070 \mathrm{~W}, n=24\end{array}$} & \multicolumn{2}{|c|}{$\begin{array}{c}9 / 08 / 2007 \\
1150 \mathrm{~W}, n=24\end{array}$} & \multicolumn{2}{|c|}{$\begin{array}{c}9 / 08 / 2007 \\
1175 \mathrm{~W}, n=24\end{array}$} & \multicolumn{2}{|c|}{$\begin{array}{c}9 / 08 / 2007 \\
1200 \mathrm{~W}, n=24\end{array}$} & \multicolumn{2}{|c|}{ All Sessions } \\
\hline Correction & $\delta^{187} \operatorname{Re}$ & \pm & $\delta^{187} \operatorname{Re}$ & \pm & $\delta^{187} \operatorname{Re}$ & \pm & $\delta^{187} \mathrm{Re}$ & \pm & $\delta^{187} \operatorname{Re}$ & \pm & $\delta^{187} \operatorname{Re}$ & \pm & $\delta^{187} \operatorname{Re}$ & \pm & $\delta^{187} \operatorname{Re}$ & \pm & $\delta^{187} \operatorname{Re}$ & \pm \\
\hline $\mathrm{S}-\mathrm{B}^{a}$ & 0.00 & 0.11 & 0.00 & 0.10 & 0.00 & 0.09 & 0.00 & 0.04 & 0.00 & 0.04 & 0.00 & 0.04 & 0.00 & 0.09 & 0.00 & 0.07 & 0.00 & 0.08 \\
\hline${ }^{186} \mathrm{~W} /{ }^{184} \mathrm{~W}^{b}$ & -2.81 & 0.04 & -2.81 & 0.04 & -2.81 & 0.03 & -2.77 & 0.06 & -2.77 & 0.03 & -2.84 & 0.03 & -2.85 & 0.03 & -2.89 & 0.03 & -2.82 & 0.07 \\
\hline${ }^{186} \mathrm{~W} / /^{183} \mathrm{~W}^{b}$ & -2.80 & 0.04 & -2.79 & 0.04 & -2.79 & 0.03 & -2.72 & 0.05 & -2.74 & 0.04 & -2.84 & 0.03 & -2.87 & 0.03 & -2.91 & 0.03 & -2.81 & 0.10 \\
\hline${ }^{186} \mathrm{~W} /{ }^{182} \mathrm{~W}^{b}$ & -2.76 & 0.03 & -2.75 & 0.03 & -2.75 & 0.03 & -2.71 & 0.03 & -2.72 & 0.03 & -2.79 & 0.03 & -2.81 & 0.03 & -2.87 & 0.03 & -2.77 & 0.08 \\
\hline${ }^{184} \mathrm{~W} /{ }^{183} \mathrm{~W}^{b}$ & -2.78 & 0.07 & -2.77 & 0.07 & -2.76 & 0.07 & -2.64 & 0.13 & -2.67 & 0.09 & -2.84 & 0.06 & -2.90 & 0.05 & -2.94 & 0.05 & -2.80 & 0.16 \\
\hline${ }^{184} \mathrm{~W} / /^{182} \mathrm{~W}^{b}$ & -2.71 & 0.04 & -2.70 & 0.04 & -2.70 & 0.03 & -2.66 & 0.07 & -2.67 & 0.03 & -2.74 & 0.04 & -2.78 & 0.03 & -2.84 & 0.03 & -2.73 & 0.10 \\
\hline${ }^{183} \mathrm{~W} /{ }^{182} \mathrm{w}^{b}$ & -2.64 & 0.08 & -2.64 & 0.06 & -2.64 & 0.07 & -2.68 & 0.17 & -2.67 & 0.08 & -2.65 & 0.07 & -2.67 & 0.06 & -2.74 & 0.07 & -2.66 & 0.09 \\
\hline Full $^{c}$ & 0.00 & 0.04 & 0.00 & 0.04 & 0.00 & 0.05 & 0.00 & 0.08 & 0.00 & 0.04 & 0.00 & 0.04 & 0.00 & 0.04 & 0.00 & 0.04 & 0.00 & 0.04 \\
\hline
\end{tabular}

Table 3.2: Values and uncertainties (2 s.d.) for 261 analyses of SRM 989 and SRM 3163 mixed standards. Solution concentrations

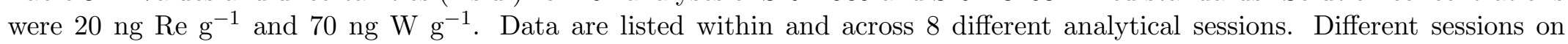
09/08/2007 feature a plasma source induced by different radio frequency (rf) generator power (1045-1200 W). For comparison, results for 8 different correction methods are listed, including standard-bracketing, W-correction according to an exponential law using all possible ratios of $\mathrm{W}$ (excepting those incorporating ${ }^{180} \mathrm{~W}$ ) with the more massive nuclide as the numerator, and a correction combining both standard-bracketing and W-correction. The full correction method appears to combine the accuracy of standard-bracketing with the precision of W-correction. See section 3.3.2 for further details. 
in the mean value of $\delta^{187}$ Re between sessions (e.g., a range of $0.12 \%$, from $-2.77 \%$ to $-2.89 \%$, for ${ }^{186} \mathrm{~W} /{ }^{184} \mathrm{~W}$-corrected $\delta^{187} \mathrm{Re}$ ). This results from the offsets between data from different analytical sessions seen in Figure 3-4. Despite the better reproducibility of W-corrected data within a given analytical session, the long-term reproducibility across analytical sessions is merely equivalent to that of standardbracketing. Because these offsets persist when examining the $\mathrm{W}$ isotopic system exclusively (Figure 3-9), they do not result from elemental differences between Re and $\mathrm{W}$, and are more likely caused by changes in instrumental mass bias from session to session. Standard-bracketing does not suffer from this marked decrease in precision across analytical sessions because ${ }^{187} \mathrm{Re} /{ }^{185} \mathrm{Re}$ is corrected with ${ }^{187} \mathrm{Re} /{ }^{185} \mathrm{Re}$ so there is no difference between correcting and corrected ratios to introduce bias. The incorporation of the accepted value for SRM 989 also has the advantage of correcting explicitly to this accepted value (i.e., 0\%o). In contrast, W-correction uses ${ }^{186} \mathrm{~W} /{ }^{184} \mathrm{~W}$ to correct ${ }^{187} \mathrm{Re} /{ }^{185} \mathrm{Re}$; because different masses are analyzed, differences in mass fractionation from session to session can introduce differences in corrected values. Also, because the measured ${ }^{187} \mathrm{Re} /{ }^{185} \mathrm{Re}$ is corrected using an assumed value for ${ }^{186} \mathrm{~W} /{ }^{184} \mathrm{~W}$ that is inappropriate, the corrected data deviate significantly from the accepted value for SRM 989.

Ideally we would want a correction method that yields accurate results relative to the accepted standard value, and is consistent across analytical sessions (like standardbracketing), while at the same time providing real-time correction for greater precision (like W-correction). We therefore use a correction method that incorporates both standard-bracketing and W-correction. Tungsten-doped samples are bracketed by $\mathrm{W}$-doped standards; correction of instrumental mass fractionation is done first by $\mathrm{W}$ correcting the measured ${ }^{187} \mathrm{Re} /{ }^{185} \mathrm{Re}$ for both the sample and bracketing standards, then by standard-bracketing the $\mathrm{W}$-corrected sample with the $\mathrm{W}$-corrected bracketing standards. Mathematically, the correction factor is: 


$$
\text { Factor }=W \text { Factor }_{\text {smpl }}\left(\frac{\left(\frac{187 R e}{185 R e} 989_{\text {std } 1} \times W \text { Factor }_{\text {std } 1}\right)+\left(\frac{187 R e}{185} \operatorname{Re} 989_{\text {std } 2} \times W \text { Factor }_{\text {std } 2}\right)}{2 \times \frac{187 R e}{185 R e} 989_{\text {true }}}\right)
$$

The results of this combined correction can be seen in Table 3.2. Corrected analyses of SRM 989 are now $0.00 \%$ and display a long-term reproducibility of $0.04 \%$.

\section{Abundance sensitivity and hydride formation}

Simultaneous analysis of Re and W may introduce analytical artifacts due to Re and $\mathrm{W}$ hydride formation and tailing. Some elements can exhibit significant hydride formation in a wet plasma. For example, CRADDOCK ET AL. (2008) calculate 1 $\times 10^{-3}$ hydride production efficiency for sulphur; $\mathrm{Re}-\mathrm{H}^{+}$or $\mathrm{W}-\mathrm{H}^{+}$contributions of this degree would be significant as isotopic variability is expected to be in the \%o range for elements of this mass (IRISAWA AND Hirata, 2006). Production efficiencies for Reand $\mathrm{W}-\mathrm{H}^{+}$have not been published, though some $\mathrm{W}$ isotopic studies specifically use a desolvating nebulizer to reduce hydride formation (e.g. HALLIDAY ET AL., 1995). This study's use of a quartz spray chamber instead of a desolvating nebulizer requires that the degree of Re and W hydride formation be characterized.

Figure 3-5 is a series of mass scans of pure SRM 989 (Fig. 3-5 A-C) and SRM 3163 (Fig 3-5 D, E) solutions obtained using the axial SEM. Solutions were evaluated

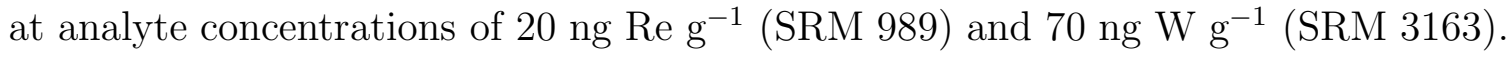

Scans over masses 184 and 186 for the SRM 989 Re standard (Fig. 3-5 A, B) show that any Re-hydride formation is swamped by an inherent SRM $989 \mathrm{~W}$ blank. The $186 / 184$ mass ratio of $\sim 1.0$ is more similar to that of ${ }^{186} \mathrm{~W} /{ }^{184} \mathrm{~W}(\sim 0.93$; VöLKENING ET AL., 1991a) than ${ }^{187} \mathrm{ReH}^{+} /{ }^{185} \mathrm{ReH}^{+}(\sim 1.67$; GRAMLICH ET AL., 1973). The extent of Re hydride formation is illustrated by the ${ }^{187} \mathrm{ReH}^{+}$contribution to mass 188 seen in Figure 3-5 C. Scans across masses 189, 190, and 192 (not shown) confirm that this signal is not ${ }^{188}$ Os. Hydride formation for Re is $\sim 1 \times 10^{-5}$ the adjacent nuclide signal, and the proportion of the mass 186 signal in Figure $3-5 \mathrm{~B}$ that is ${ }^{185} \mathrm{ReH}^{+}$is $\sim 7 \%$. 

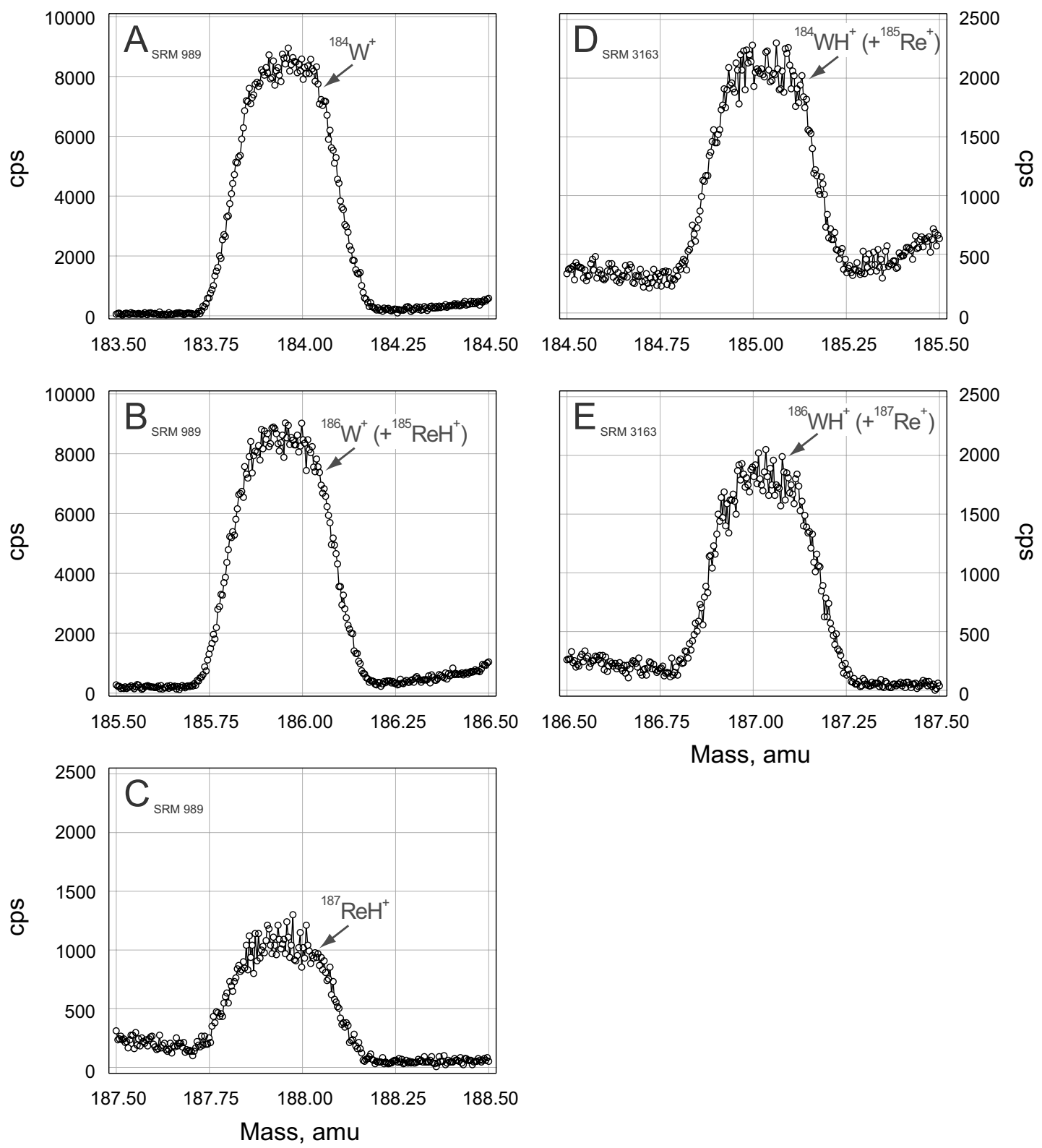

Figure 3-5: Axial SEM scans of relevant $\mathrm{W}$ and Re masses for separate (not mixed) solutions of SRM 989 (A-C; $20 \mathrm{ng} \mathrm{Re}^{-1}$ ) and SRM 3163 (D, E; $70 \mathrm{ng} \mathrm{W} \mathrm{g}{ }^{-1}$ ). See Section 3.3.2 for discussion.

A: Scan across mass 184 for SRM 989 showing the SRM 989 W blank.

B: Scan across mass 186 for SRM 989 showing the SRM 989 W blank.

C: Scan across mass 188 for SRM 989 illustrating the degree of Re-hydride formation.

D: Scan across mass 185 for SRM 3163 illustrating the degree of W-hydride formation.

E: Scan across mass 187 for SRM 3163 illustrating the degree of W-hydride formation. 
The isotope standard SRM 989 has a W blank with a Re/W ratio of $\sim 4300$. At these levels, the $\left({ }^{186} \mathrm{~W}+{ }^{185} \mathrm{ReH}^{+}\right) /{ }^{184} \mathrm{~W}$ ratio would have to differ from the accepted ${ }^{186} \mathrm{~W} /{ }^{184} \mathrm{~W}$ value (VÖLKENING ET AL., 1991a) by more than $\pm 83 \%$ o to perturb the corrected value of SRM 989 by more than $\pm 0.01 \%$.

Formation of W-hydrides is seen in Figures 3-5 D and E. The corresponding $187 / 185$ mass ratio of $\sim 1.0$ is more similar to that of ${ }^{186} \mathrm{WH}^{+} /{ }^{184} \mathrm{WH}^{+}$than ${ }^{187} \mathrm{Re} /{ }^{185} \mathrm{Re}$. Hydride formation of $\mathrm{W}$ is $\sim 2 \times 10^{-5}$ the adjacent nuclide signal. At the analyte concentrations used, the contributions of ${ }^{184} \mathrm{WH}^{+}$and ${ }^{186} \mathrm{WH}^{+}$to the ${ }^{185} \mathrm{Re}$ and ${ }^{187} \mathrm{Re}$ peaks are well within the signal noise $(>0.1 \%, 1$ s.d.). The production efficiencies of Re and W hydride formation of $1-2 \times 10^{-5}$ observed in this study are comparable to those of observed for uranium (VAIS ET AL., 2004) and do not affect corrected $\delta^{187}$ Re values at significant levels.

Figure 3-5 also shows evidence for tailing of both $\mathrm{W}$ and Re (e.g. Fig. 3-5 shows up-mass tailing of ${ }^{184} \mathrm{~W}$ and down-mass tailing of ${ }^{186} \mathrm{~W}$ into mass 185 ) though at levels much lower than hydride formation or blank concentration. The down-mass abundance sensitivity of $\mathrm{W}$ and $\mathrm{Re}$ is $\sim 2-4 \mathrm{ppmamu}^{-1}$; up mass, the abundance sensitivity is $1 \mathrm{ppmamu}^{-1}$.

\subsection{Rhenium isotopic variability in commercially available materials}

We evaluate the isotopic composition of a series of commercially available Re materials: SRM 989, SRM 3143, an Alfa Aesar Specpure ${ }^{\circledR}$ Re standard, a zone-refined Re ribbon from H. Cross Co., $\mathrm{HReO}_{4}$, and the Devonian Ohio Shale standard USGS SDO-1 39 (Table 3.4). A total range in Re isotopic composition of $\sim 0.3 \%$ is observed. The Ohio Shale standard USGS SDO-1 has a $\delta^{187}$ Re value of $0.21 \%$. The presence of isotopic variability in the materials analyzed is encouraging to future research of Re isotopic composition of natural materials. 


\begin{tabular}{|c|c|c|c|c|c|}
\hline Standard ID & $\operatorname{ng} \operatorname{Reg}^{-1}$ & $\mathrm{ng} \mathrm{Wg}^{-1}$ & $\mathrm{n}$ & $\delta^{187} \operatorname{Re}$ & \pm \\
\hline SRM 989 & 20 & 70 & 281 & 0.00 & 0.04 \\
\hline SRM 989 & 10 & 35 & 22 & 0.02 & 0.07 \\
\hline SRM 3143 & 10 & 35 & 22 & 0.29 & 0.07 \\
\hline $\mathrm{HReO}_{4}$ & 10 & 35 & 22 & 0.22 & 0.09 \\
\hline Alfa Aesar* & 10 & 35 & 20 & 0.01 & 0.10 \\
\hline H. Cross ribbon & 10 & 35 & 22 & 0.28 & 0.12 \\
\hline USGS SDO-1 & 10 & 35 & 5 & 0.21 & 0.13 \\
\hline
\end{tabular}

* Refers to Alfa Aesar Specpure ${ }^{\circledR}$ Lot \#235760A

Table 3.3: Summary of values and uncertainties (2 s.d.) for various standard materials and in-house standards. Uncertainties at analyte concentrations of $10 \mathrm{ng} \mathrm{Re} \mathrm{g}^{-1}$ and $35 \mathrm{ng} \mathrm{W}$ $\mathrm{g}^{-1}$ is $\sim 0.10 \%$. Doubling the concentrations to $20 \mathrm{ng} \mathrm{Re}^{-1}$ and $70 \mathrm{ng} \mathrm{W} \mathrm{g}{ }^{-1}$ halves the uncertainty to $\sim 0.05 \%$. Data for SDO-1 represent five analyses of three separate sample treatments.

\subsection{Concerns regarding natural samples}

To this point we have examined Re isotopes mainly in clean standard solutions. For the Re system to be of real value, analyses must be extended to natural samples such as SDO-1. The analysis of geochemical materials introduces a host of additional concerns such as analyte requirements, the potential for fractionation during sample processing, matrix effects, and requisite purification of Re from Os.

\subsubsection{Analyte requirements}

The desired minimum $\delta^{187}$ Re precision $\pm 0.10 \%$ requires $1 \mathrm{~g}$ of solution at $10 \mathrm{ng}$

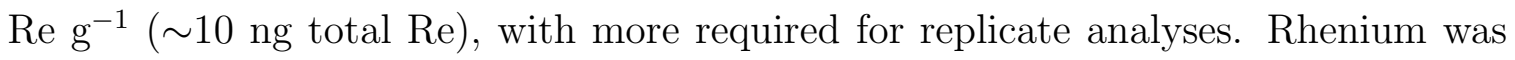
the last stable chemical element discovered (NODDACK ET AL., 1925; NoDDACK AND NoDDACK, 1931) mainly because of its scarcity in crustal materials (NoDDACK Et Al., 1925; Noddack And Noddack, 1931; Esser And Turekian, 1993; MC- 
Donough And Sun, 1995; Hauri And Hart, 1997) and the rarity with which it forms its own minerals (CAPITANT ET AL., 1963; Morris AND Short, 1966; Mitchell et AL., 1989; TARkian et AL., 1991; Korzhinski Et Al., 1994; Power ET AL., 2004). Figure 3-6 details the amount of sample required to yield $30 \mathrm{ng}$ total $\mathrm{Re}$, enough for triplicate analyses at a Re solution concentration of $10 \mathrm{ng} \operatorname{Re} \mathrm{g}^{-1}$. Many sample types would require processing of inconveniently large quantities of

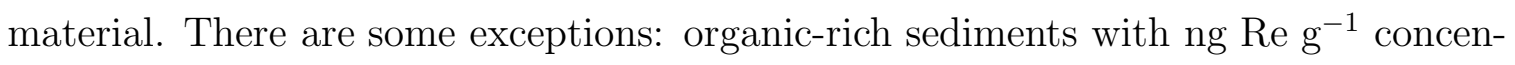
trations (Ravizza and Turekian, 1989; Ravizza et al., 1991; Cohen et al., 1999; Peucker-Ehrenbrink and Hannigan, 2000; Creaser et al., 2002) and molybdenites $\left(\mathrm{MoS}_{2}\right)$ with concentrations from the ppm to \% range (FLEISCHER, 1959, 1960; Morachevski and Nechaeva, 1960; Bernard et al., 1990). Despite generally low Re concentrations (Colodner et AL., 1993; DAlai Et AL., 2002, Chapter 2) some natural waters can be analyzed despite the large substrate mass required (e.g., $4.2 \mathrm{~kg}$ of seawater), as Re is already in solution.

\subsubsection{Chromatographic fractionation}

It is widely known that chromatographic methods can fractionate isotopes (RUSSEL ET AL., 1978; Anbar et AL., 2000; Roe ET Al., 2003), even for elements in the Re mass range (IRISAWA AND HiRATA, 2006). Figure 3-7 provides details of chromatographic recovery and isotopic fractionation of Re. Recovery of Re is complete within

uncertainty, and though fractionation does occur, the $\delta^{187} \operatorname{Re}$ of the cumulative eluate converges with the standard value after elution of $80 \%$ of the total Re, or after $70 \%$ of the total eluate. Initial eluate is enriched in ${ }^{187} \mathrm{Re}$, with $\delta^{187} \mathrm{Re}$ of at least $+1.2 \%$, before becoming progressively depleted. Several other studies note enrichment of the heavier isotope in the initial eluate (Russel Et AL., 1978; AnBAR Et AL., 2000; Roe et Al., 2003; Irisawa And Hirata, 2006). After reaching the bulk value, later eluate does not appear to be comparably depleted as has been seen in other studies (ANBAR ET AL., 2000; Roe ET AL., 2003) 


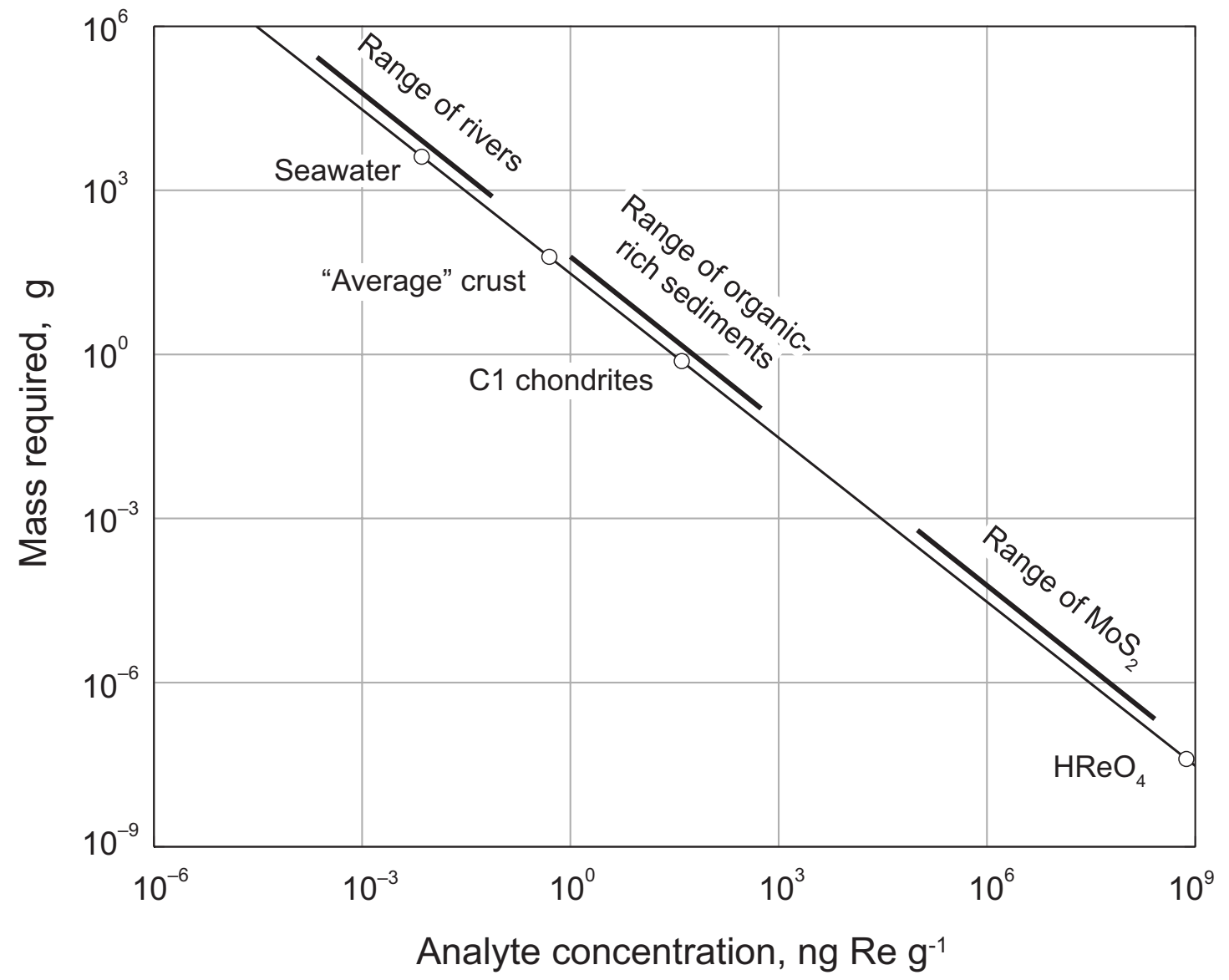

Figure 3-6: Plot of the relationship between substrate Re concentration and the amount of substrate required for analysis. The $30 \mathrm{ng}$ contour is plotted along with data regarding specific sample types.

Data are from NodDack ANd NodDack (1931); Fleischer (1959); Morachevskil AND Nechaeva (1960); Terada et Al. (1971); Anders and Grevesse (1989); Ravizza and Turekian (1989); Bernard et Al. (1990); Ravizza et Al. (1991); Colodner Et AL. (1993); Esser and Turekian (1993); MCCAndless et Al. (1993); Korzhinski et Al. (1994); Markey et Al. (1998); Cohen et Al. (1999); Peucker-Ehrenbrink and Hannigan (2000); Selby and Creaser (2001); Creaser et al. (2002); Dalai Et Al. (2002); Barra et Al. (2003); MaO et Al. (2003); Berzina et Al. (2005); MaO ET AL. (2006). 

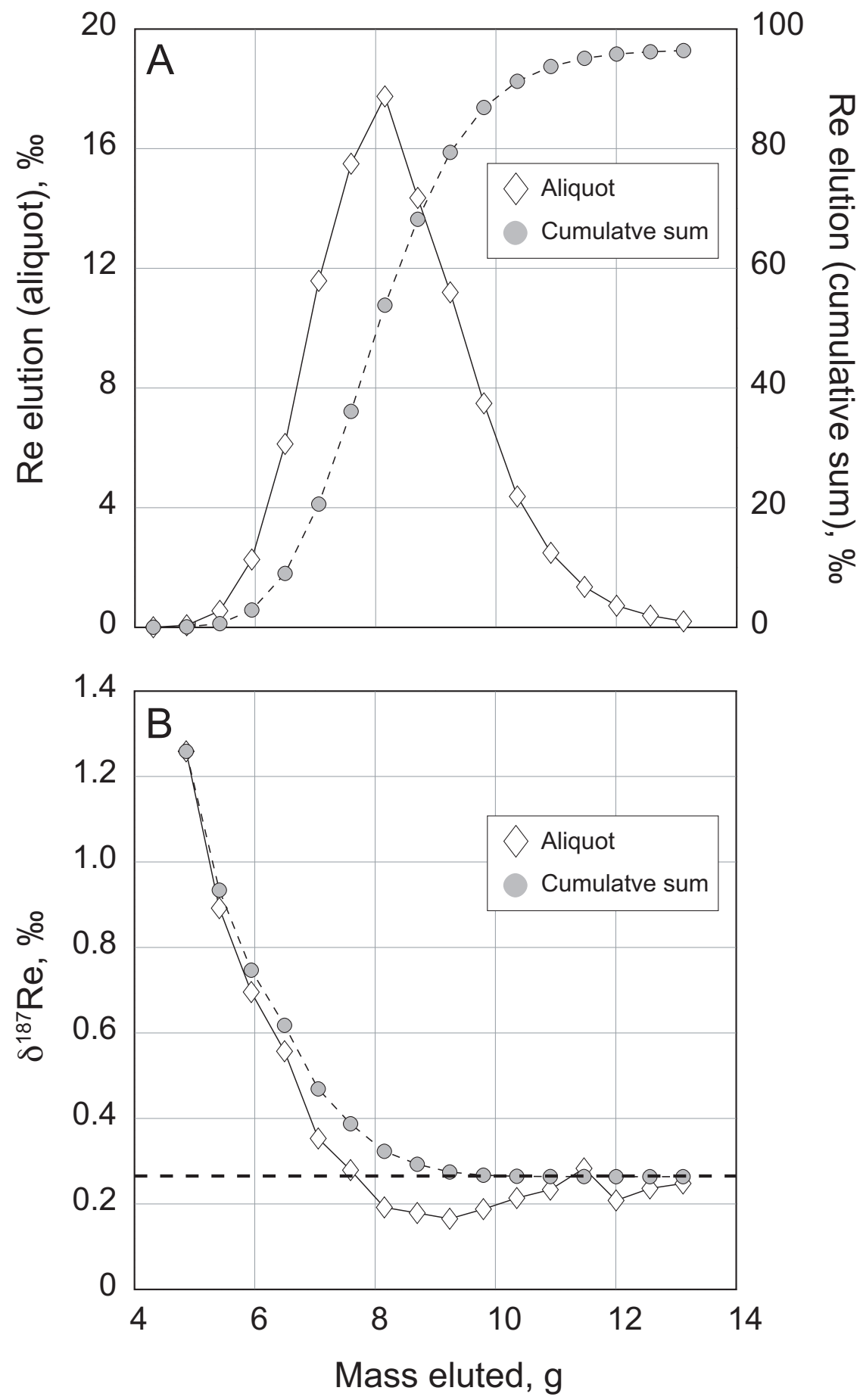

Figure 3-7: Effects of column chromatographic purification for standard Re.

A: Aliquot and cumulative proportions of recovered Re with increasing mass of eluent eluted. B: Aliquot and cumulative $\delta^{187}$ Re with increasing eluted eluent mass. Cumulative $\delta^{187} \operatorname{Re}$ converges with the standard value (dashed line) after elution of $\sim 80 \%$ of total Re. 


\subsubsection{Matrix effects}

Though chromatographic recovery of Re is quantitative, it does not result in complete separation from other elements. These other elements constitute the sample matrix (i.e., what is introduced to the ion source) and are frequently present in much higher abundance than Re. These elements can affect the data as interference on our nuclides of interest, or by affecting the mass bias through space-charge effects.

To test matrix effects, we obtained mass scans from 7 to 230 amu for a 100fold dilution of black shale matrix; see Figure 3-8. In addition to Re, the matrix contains significant Fe, Zn, Zr, Ba, and Hf, as well as many other elements at much lower abundance. Using the known concentration of Re in this solution and assuming ionization equivalent to that of Re, rough concentrations for these elements were determined, and an artificial solution was created incorporating all identifiable matrix elements except Re. Standard Re of known isotopic composition was then added to the artificial matrix prior to analysis. No difference between clean and artificial matrix standards could be discerned. The black shale matrix does not compromise the accuracy and precision of the Re isotope data.

\subsubsection{Decay to Os and sparging}

${ }^{187}$ Rhenium is unstable and $\beta^{-}$-decays with $\lambda=1.667(3) \times 10^{-11} \mathrm{yr}^{-1}$ to its isobar, ${ }^{187}$ Os (SELBY ET AL., 2007) requiring the removal of all ${ }^{187}$ Os before analysis. Fortunately, Os forms a volatile compound, $\mathrm{OsO}_{4}^{0}$, that can be exploited for separation from Re.

Sample chemistry and purification involves dissolution/leaching in highly oxidizing media such as aqua regia and concentrated $\mathrm{HNO}_{3}$, as well as repeated dry-down steps all of which serve to promote oxidation to $\mathrm{OsO}_{4}^{0}$. A clean isotope of Os is nonetheless monitored to identify samples that may need further purification. ${ }^{189}$ Osmium is monitored as ${ }^{184} \mathrm{Os},{ }^{186} \mathrm{Os},{ }^{187} \mathrm{Os}$ have elemental $\mathrm{W}$ and Re interferences, acquisition of ${ }^{188} \mathrm{Os}$ is not permitted by the architecture of the collector array, and ${ }^{190} \mathrm{Os}$ 

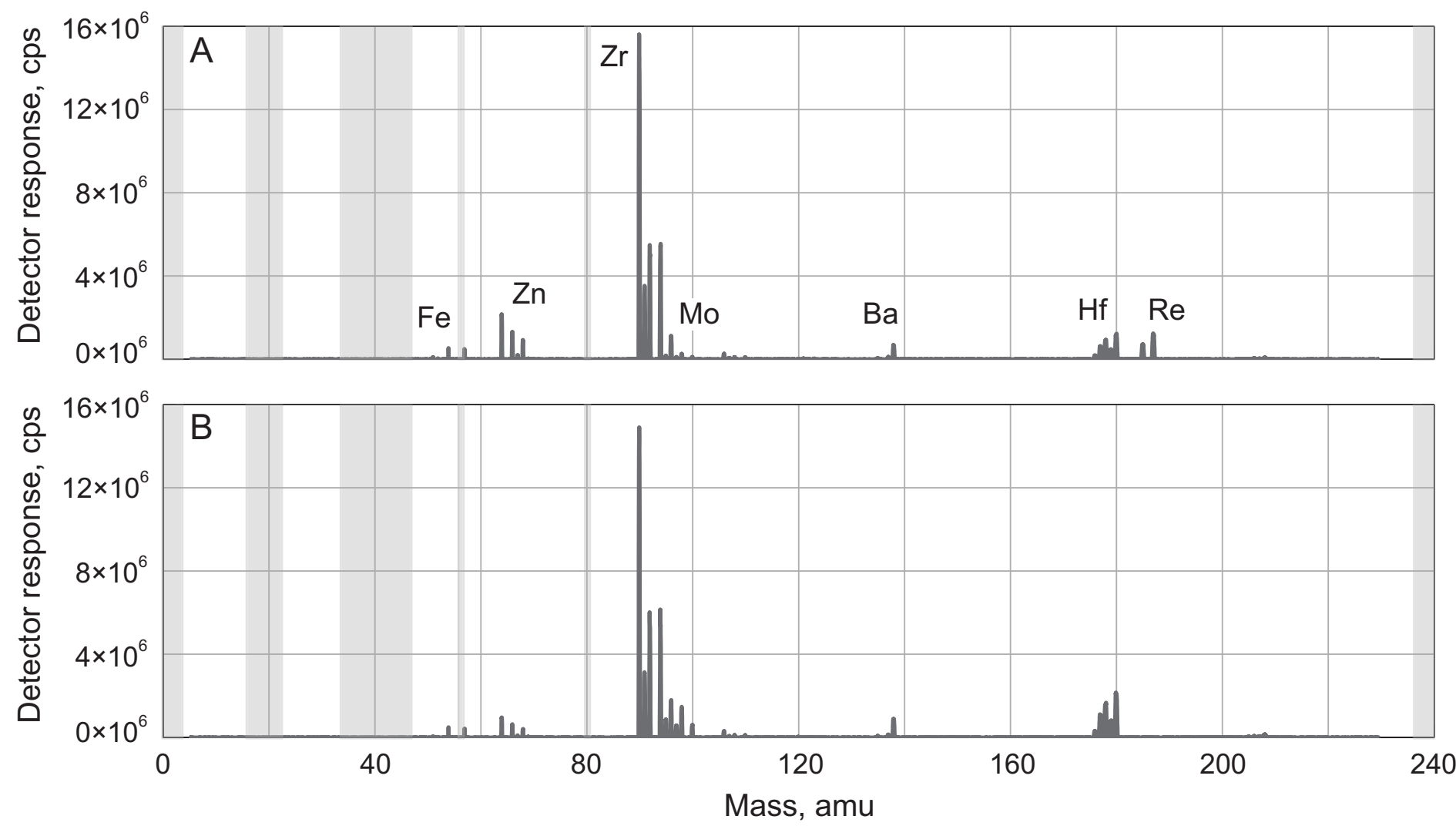

Figure 3-8: Mass scan from 7-230 amu for actual (A) and artificial (B) black shale matrices (single-collector ICPMS, Thermo Fisher Scientific ELEMENT 2). Areas in grey are blocked out of the scanning protocol to protect the SEM.

A: Mass scan of a 100-fold dilution of an actual black shale sample matrix showing a variety of matrix elements at concentrations comparable to or exceeding that of Re. Magnifications of the 179-187 amu mass range (not shown) indicates that W is present in negligible quantities.

B: An identical scan of a 100-fold dilution of an artificial solution lacking Re, but otherwise deliberately synthesized to resemble the natural black shale matrix. 
and ${ }^{192} \mathrm{Os}$ frequently have respective molecular ${ }^{174} \mathrm{Hf}^{16} \mathrm{O}^{+}$and ${ }^{176} \mathrm{Hf}^{16} \mathrm{O}^{+}$interferences (Section 3.5.3). If the Os isotopic composition of the sample is known, ${ }^{189}$ Os data can also be used to correct measured ${ }^{184} \mathrm{~W},{ }^{186} \mathrm{~W}$, and ${ }^{187} \mathrm{Re}$, allowing a mass bias correction despite the presence of Os. However, this is not ideal as Re and W data collection is interspersed with ${ }^{189}$ Os collection by peak-hopping over the course of a sample analysis. In addition, some substrates, such as commercially available standards and some sulfide minerals, are highly enriched in Re but not Os; if samples are sufficiently old, this can result in significant ${ }^{187}$ Os without the presence of ${ }^{189} \mathrm{Os}$ to indicate contamination. Such samples require a method designed to purify Re whether we know if Os is present or not.

Development of "sparging," an analytical method in which Os is stripped from an oxidising solution with Ar gas and introduced directly into an ICPMS source (RUSS ET AL., 1987; Gregoire, 1990; Hassler ET AL., 2000) provides both a method for sample purification and the means of evaluating it. Figure 3-8 shows data for a sparging experiment. A mixed SRM 989 and SRM 3163 standard in 4 mol L-1 $\mathrm{HNO}_{3}$ was doped with Os with a ${ }^{187} \mathrm{Os} /{ }^{188}$ Os of 0.1069 and heated for several hours in a closed Teflon ${ }^{\circledR}$ vial at $100^{\circ} \mathrm{C}$ to promote the oxidation of Os to $\mathrm{OsO}_{4}$. The solution was sparged for over six hours during which data were taken continuously at eight second intervals (2800 total measurements). The exponential decrease in the Os signal shows that sparging is an effective means of removing Os from solution. The initial decrease in Os (Fig. 3-8 A) indicates a "half-life" of $~ 18$ minutes within the sparging solution. At approximately 2.5 hours the Os loss rate decreases, and the Os signal intensity approaches some constant value. Another heating-sparging cycle produced very little additional Os, indicating that the non-zero final Os signal reflects an increased background due to the processing of $\sim 85 \mathrm{ng}$ of Os through the sample inlet system. Removal of Os is also seen in the exponential decrease of measured ${ }^{187} \mathrm{Re} /{ }^{185} \mathrm{Re}$ and ${ }^{186} \mathrm{~W} /{ }^{183} \mathrm{~W}$ ratios, and in the increased variability of ${ }^{192} \mathrm{Os} /{ }^{190} \mathrm{Os}$ seen later in the experiment. 

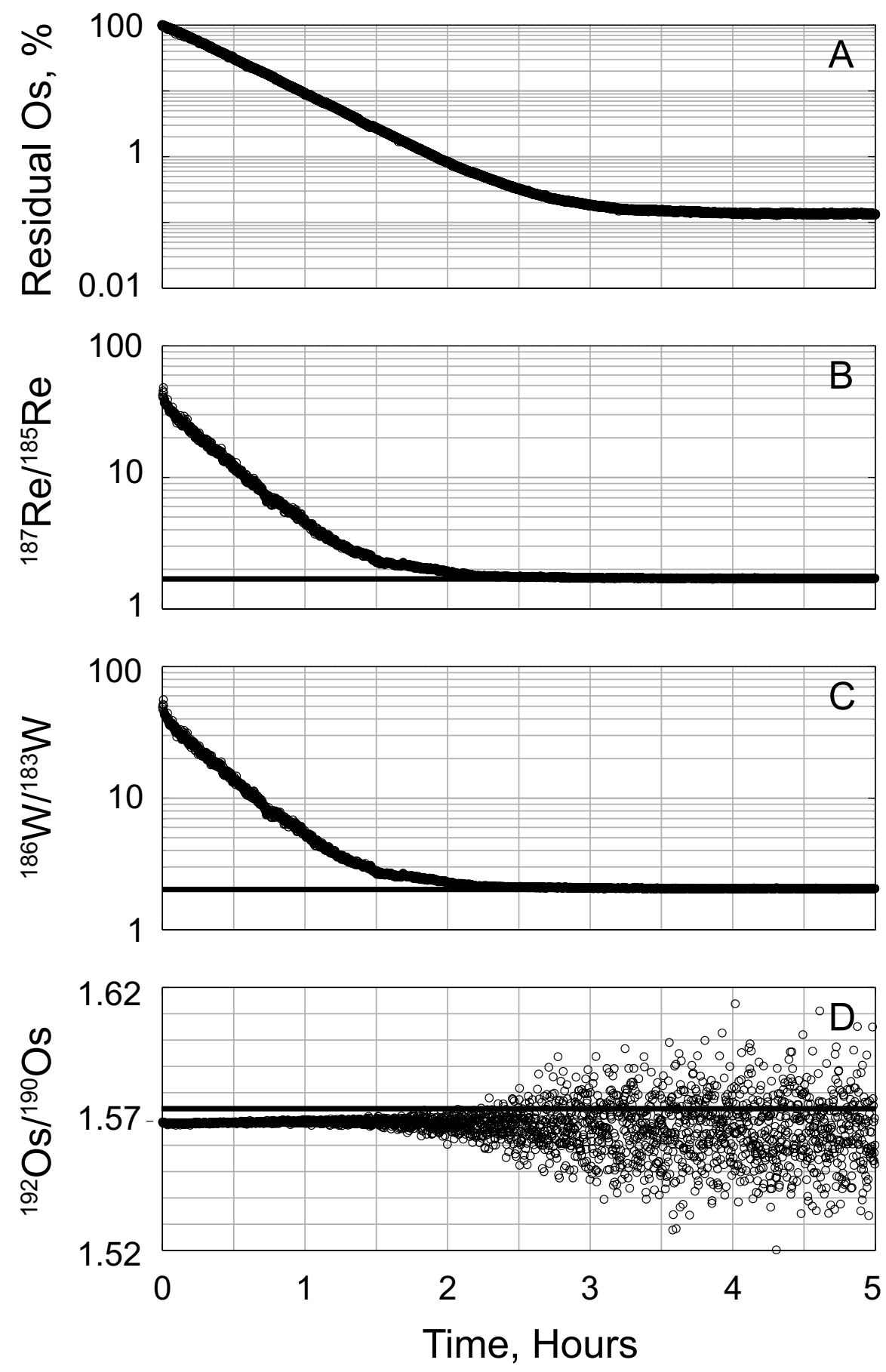

Figure 3-8: Full caption on page 157. 
Figure 3-8: Residual Os, ${ }^{187} \mathrm{Re} /{ }^{185} \mathrm{Re},{ }^{186} \mathrm{~W} /{ }^{184} \mathrm{~W}$, and ${ }^{192} \mathrm{Os} /{ }^{190} \mathrm{Os}$ data from a $5 \mathrm{hr}$ sparg-

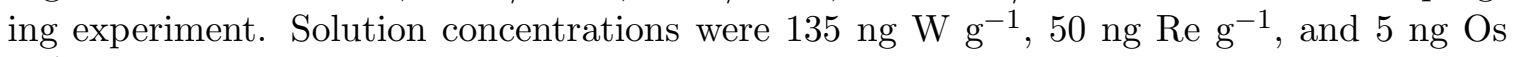
$\mathrm{g}^{-1}$. These concentrations were chosen to allow collection of all three elements using the Faraday cups in static mode. The total solution mass sparged was $\sim 17 \mathrm{~g}$.

A: A plot of the proportion of Os remaining, expressed as a percentage of the initially ion beam intensity.

B: A plot of measured ${ }^{187} \mathrm{Re} /{ }^{185} \mathrm{Re}$ which also shows an exponential decrease (due to the removal of ${ }^{187} \mathrm{Os}$ ) before converging with its natural value (solid line).

C: A plot of measured ${ }^{186} \mathrm{~W} /{ }^{183} \mathrm{~W}$ exhibiting similar behavior to that of ${ }^{187} \mathrm{Re} /{ }^{185} \mathrm{Re}$; ${ }^{186} \mathrm{~W} /{ }^{184} \mathrm{~W}$ was not monitored as the architecture of the detector array prevented the acquisition of ${ }^{184} \mathrm{~W}$.

D: A plot of ${ }^{192} \mathrm{Os} /{ }^{190} \mathrm{Os}$; note that the vertical axis is linear. Also note that the dark line indicates the expected values of ${ }^{192} \mathrm{Os} /{ }^{190} \mathrm{Os}$ - that is, the accepted ratio value after a plausible degree of mass fractionation has been artificially imposed. ${ }^{192} \mathrm{Os} /{ }^{190}$ Os data are initially precise and consistent with the expected value, but start to vary significantly as Os intensity decreases late in the experiment.

Interference from Os is not a concern for standard data presented herein as all standards were sparged without showing any change in isotope ratio.

\subsection{Conclusions}

This study investigates the application of MC-ICPMS to the Re isotopic system. The most precise ( $\pm 0.04 \%$ o 2 s.d.) and accurate data are obtained using a combination of standard-bracketing with external correction using ${ }^{186} \mathrm{~W} /{ }^{184} \mathrm{~W}$ and an exponential fractionation law to correct for instrumental mass fractionation. We observe $0.3 \%$ variability in Re isotope composition across three commercially available Re standards, two in-house Re standards, and the Devonian Ohio shale standard USGS SDO-1.

Application of this isotope system to natural samples is complicated by the low abundance of Re in most crustal materials, which limits the substrates that can be conveniently analyzed. However, there are still suitable sample substrates available; organic-rich sediments and sulfides are examples. Chromatographic purification can 
induce more than $1 \%$ of isotopic fractionation in the absence of quantitative recovery. Despite high abundances of other elements in the sample matrix, the Re system does not appear to exhibit matrix-related isotopic variability. Complete separation of Re from Os is required by the isobaric $\beta^{-}$-decay of ${ }^{187} \mathrm{Re}$ to ${ }^{187} \mathrm{Os}$. This is achieved in the oxidizing environment present throughout the sample preparation process, and through sparging if necessary.

\subsection{Acknowledgments}

We would like to acknowledge financial support from NSF-EAR grant 0519387 and from the WHOI Academic Programs Office, as well as support for the WHOI Plasma Mass Spectrometry Facility from NSF-EAR/IF grants 0318137 and 0651366. We would also like to thank the members of the WHOI Plasma Mass Spectrometry Facility: Scot Birdwhistell, Jerzy Blusztajn, and Dave Schneider. Brad Esser of LLNL and Bob Vocke of NIST provided standard materials. This manuscript was greatly improved through discussion with our WHOI colleagues Dave Glover and Olivier Rouxel, as well as with Travis Poole, University of Edinburgh. 


\section{A Mass fractionation in the $\mathrm{W}$ system}

Studies employing external correction have noted that differences between predicted and observed behavior are due to chemical differences (HIRATA, 1996; MARÉchaL ET AL., 1999). The use of $\mathrm{W}$ to correct Re for instrumental mass bias provides an opportunity to evaluate fractionation systematics within a single elemental system, that is, it allows us to determine whether deviations are due to mass differences as opposed chemical differences between Re and W.

Figures 3-4 and 3-9 allow us to compare fractionation for Re vs. W and within the W system, respectively. Firstly, all instrumentally-fractionated ratio pairs are highly correlated. Secondly, slopes obtained from the type II linear regressions do not overlap consistently with those obtained from any particular empirical fractionation law; see also Tables 3.1 and 3.4 (also note that slopes in Table 3.4 are positive as the heavier nuclide is always in the numerator while equivalent slopes in Figure 3-9 are negative as all nuclides, regardless of mass, are normalized to ${ }^{184} \mathrm{~W}$ in order to obtain a three-isotope plot). Finally, in most cases, data from different analytical sessions are clearly distinguishable from one another, particularly for those obtained using variable rf generator power.

The correlation and scatter about the best-fit regressions are no worse for Re-W than for $\mathrm{W}-\mathrm{W}$. The essentially equivalent degrees of correlation and scatter are surprising given that the $\mathrm{W}-\mathrm{W}$ data are three-isotope plots for a single elemental system, while the Re-W data is a four-isotope plot incorporating two elemental systems; this suggests that there is little difference between the fractionation behaviors of Re and W.

A lack of consistency between observed (type II regression slopes) and expected (fractionation law slopes) fractionation occurs not only for ${ }^{187} \mathrm{Re} /{ }^{185} \mathrm{Re}$ vs. ${ }^{186} \mathrm{~W} /{ }^{184} \mathrm{~W}$, but also for ${ }^{183} \mathrm{~W} /{ }^{184} \mathrm{~W}$ vs. ${ }^{186} \mathrm{~W} /{ }^{184} \mathrm{~W}$ and ${ }^{182} \mathrm{~W} /{ }^{184} \mathrm{~W}$ vs. ${ }^{186} \mathrm{~W} /{ }^{184} \mathrm{~W}$. As a result, deviations from behavior predicted by the empirical fractionation laws cannot be the result of chemical differences between Re and W. This is unsurprising given the 

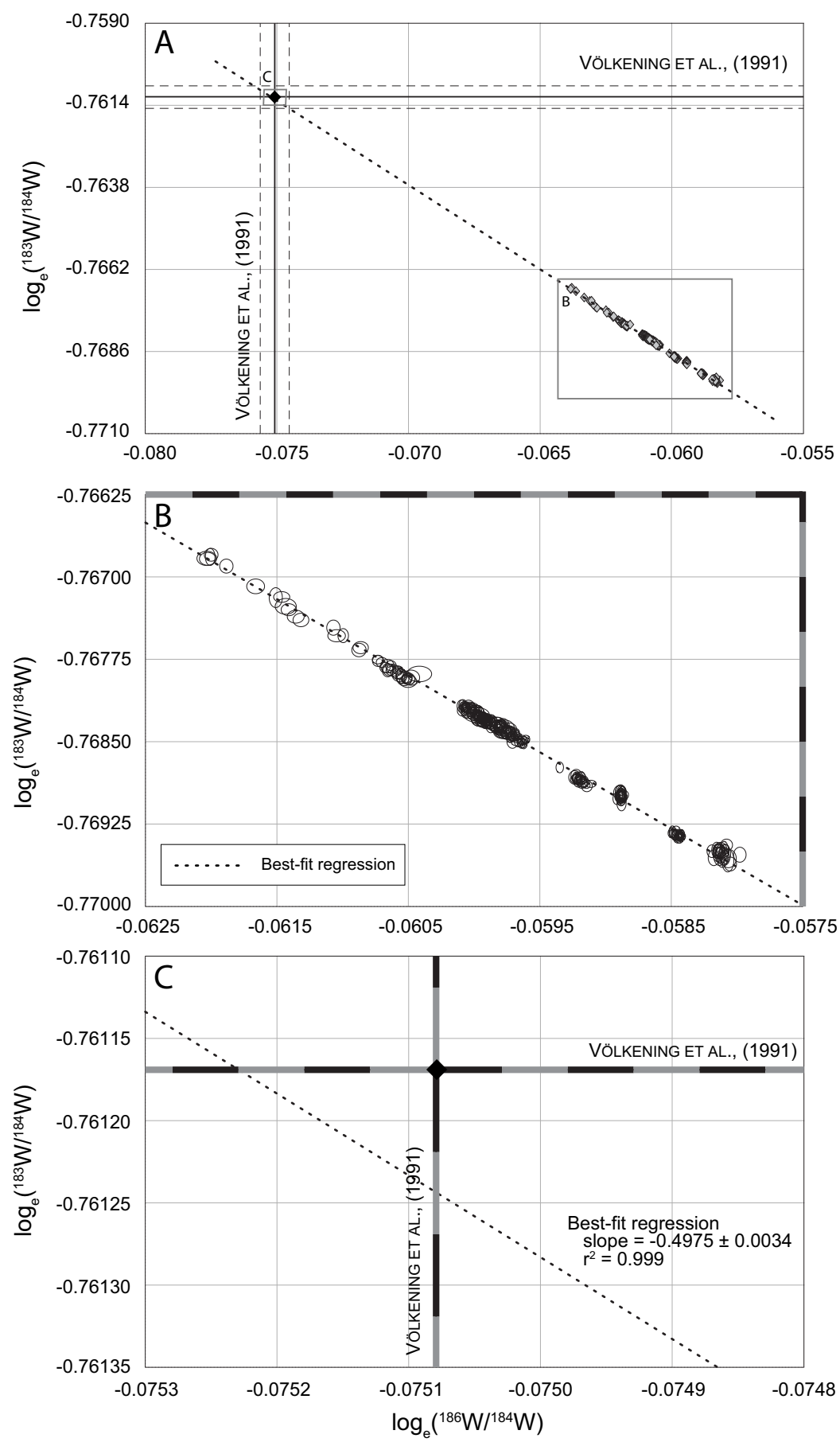

Figure 3-9: Full caption on page 162. 

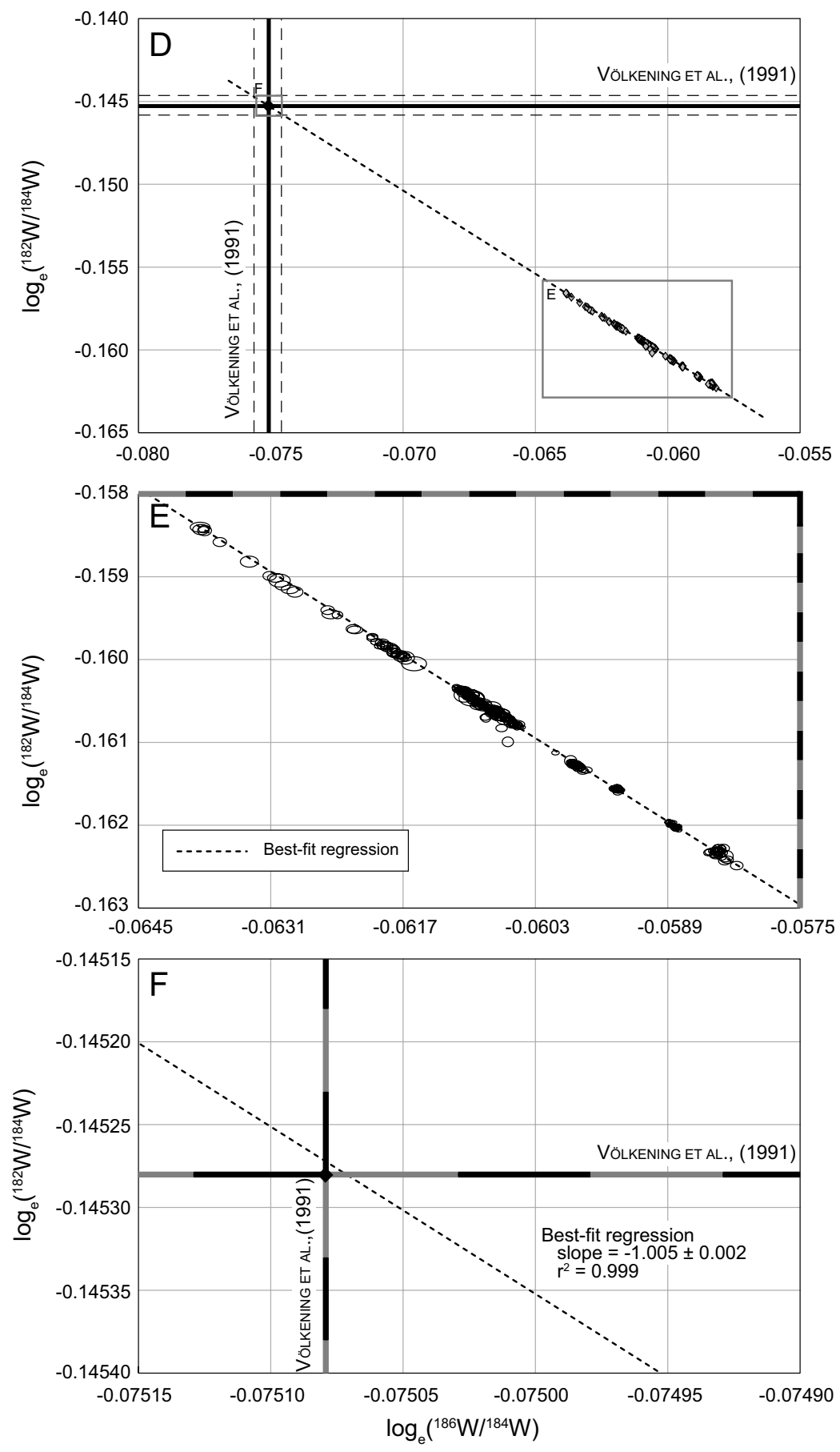

Figure 3-9: Full caption on page 162. 
Figure 3-9: Three-isotope diagrams of ${ }^{183} \mathrm{~W} /{ }^{184} \mathrm{~W}$ vs. ${ }^{186} \mathrm{~W} /{ }^{184} \mathrm{~W}$ (page 160 ) and ${ }^{182} \mathrm{~W} /{ }^{184} \mathrm{~W}$ vs. ${ }^{186} \mathrm{~W} /{ }^{184} \mathrm{~W}$ (page 161) in $\log _{e}$ space. Diagrams incorporating ${ }^{180} \mathrm{~W}$ are not included due to the much lower precision of ratios incorporating this nuclide.

A: ${ }^{183} \mathrm{~W} /{ }^{184} \mathrm{~W}$ vs. ${ }^{186} \mathrm{~W} /{ }^{184} \mathrm{~W}$ data as well as accepted values with 2 s.d. uncertainties (VÖLKENING ET AL., 1991a) and observed fractionation trends - obtained by a best-fit type II linear regression. Linear, power, Rayleigh, and exponential fractionation trends are not shown, though they do overlap very well with the data and observed fractionation trends.

B: A magnification of the area corresponding to an instrumental mass bias of $+0.75 \%$ $\mathrm{amu}^{-1}$. Scale bars at top and right are $0.5 \%$. ${ }^{183} \mathrm{~W} /{ }^{184} \mathrm{~W}$ vs. ${ }^{186} \mathrm{~W} /{ }^{184} \mathrm{~W}$ data from individual analytical sessions appear as data clusters. Data from individual sessions also show offsets relative to the best-fit regression line; in many cases these offsets are produced by differing radio frequency power (rf) and can result in $0.1 \%$-level differences in corrected $\delta^{183} \mathrm{~W} /{ }^{184} \mathrm{~W}$ values.

C: A magnification of the area of accepted isotopic ratio values for SRM 3163. Scale bars are $0.05 \%$ ( $50 \mathrm{ppm})$. The intersections of the observed fractionation trend and accepted values for SRM 3163 give corrected values within the quoted uncertainties. Note however, that accepted ratio uncertainties lie beyond the figure boundary.

D: Same as (A), but for ${ }^{182} \mathrm{~W} /{ }^{184} \mathrm{~W}$ vs. ${ }^{186} \mathrm{~W} /{ }^{184} \mathrm{~W}$.

E: Same as (B), but for ${ }^{182} \mathrm{~W} /{ }^{184} \mathrm{~W}$ vs. ${ }^{186} \mathrm{~W} /{ }^{184} \mathrm{~W}$.

F: Same as (C), but for ${ }^{182} \mathrm{~W} /{ }^{184} \mathrm{~W}$ vs. ${ }^{186} \mathrm{~W} /{ }^{184} \mathrm{~W}$. 
very similar first ionization potentials of these elements (CAMPBELL-Miller AND Simard, 1996). Such effects cannot be ruled out a priori, however, as other combinations of correcting-corrected elements may have first ionization potentials that differ to a larger degree, e.g., Zn-Cu (MarÉchal et AL., 1999). Hirata (1996), ascribe deviations in mass fractionation to differences in the masses of correcting and corrected ratios, but consideration of our data does not confirm that this applies to analyses of Re+W. Careful observation of Table 3.4 does not show an increased overlap between observed and expected fractionation with increasing similarity in mass between correcting and corrected ratios (this would be seen as a clustering of bold fractionation trends about the diagonal). Quite simply, none of the empirical fractionation laws fully describe mass fractionation and choice of correcting law must be made on a case by case basis.

Major differences are not seen by comparing Re-W to W-W data, but rather by comparing data from different analytical sessions. Differences are particularly obvious for six different analytical sessions from a single day that differ only in the rf generator power used to induce the plasma. These data suggest that plasma conditions should be standardized as they noticeably affect the instrumental mass fractionation in MCICPMS. 
Correcting Ratios

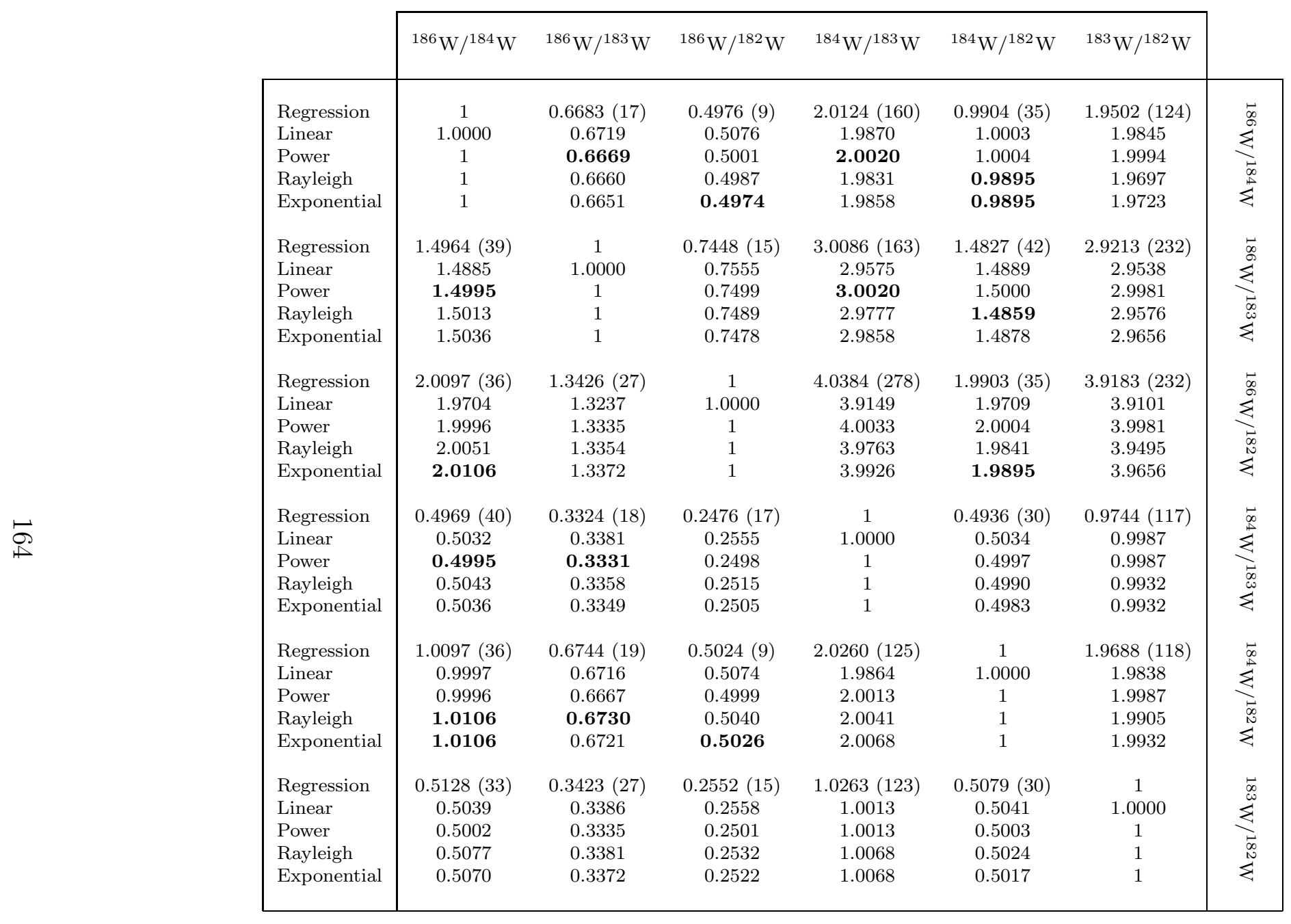

Table 3.4: Observed and expected fractionation trends for $\mathrm{W}$ isotopic fractionation in $\log _{e}$ space. Expected fractionation trends calculated as described in Table 3.1. Uncertainties are included with the best-fit regressions (2 s.d.). Factors shown in bold-face type are within the uncertainty of the best-fit regression. Also note that the information on either side of the diagonal is redundant (e.g., the Rayleigh fractionation factor for the correction of ${ }^{183} \mathrm{~W} /{ }^{182} \mathrm{~W}$ with ${ }^{186} \mathrm{~W} /{ }^{184} \mathrm{~W}$ is simply the inverse of the Rayleigh fractionation factor for the correction of ${ }^{186} \mathrm{~W} /{ }^{184} \mathrm{~W}$ with ${ }^{183} \mathrm{~W} /{ }^{182} \mathrm{~W}$. 


\section{Bibliography}

Anbar, A. D., Roe, J. E., and Nealson, K. H. (2000), Nonbiological fractionation of iron isotopes, Science, 288: 126-128. [150]

Anders, E. and Grevesse, N. (1989), Abundances of the elements: Meteoritic and solar, Geochimica et Cosmochimica Acta, 54: 197-214. [151]

Arnold, G. L., Anbar, A. D., Barling, J., and Lyons, T. W. (2004), Molybdenum isotope evidence for widespread anoxia in mid-Proterozoic oceans, Science, 304: 87-90. [16, 22, 98, 104, 129, 175, 176, 186, 198, 199]

Audi, G. and Wapstra, A. H. (1995), The 1995 update to the atomic mass evaluation, Nuclear Physics A, 595: 409-480. [126]

Barra, F., Ruiz, J., Mathur, R., and Titley, S. (2003), A Re-Os study of sulfide minerals from the Bagdad porphyry $\mathrm{Cu}-\mathrm{Mo}$ deposit, northern Arizona, USA, Mineralium Deposita, 38: 585-596. [78, 151]

Beard, B. L., Johnson, C. M., Cox, L., Sun, H., Nealson, K. H., and Aguilar, C. (1999), Iron isotope biosignatures, Science, 285: 1889-1892. [129]

Bergquist, B. A. and Blum, J. D. (2007), Mass-dependent and -independent fractionation of $\mathrm{Hg}$ isotopes by photoreduction in aquatic systems, Science, 318: 417-420. [129]

Bernard, A., Symonds, R. B., and Rose, W. I. J. (1990), Volatile transport of Mo, W, and Re in high temperature magmatic fluids, Applied Geochemistry, 5: 317-326. $[150,151]$

Berzina, A. N., Sotnikov, V. I., Economou-Eliopoulos, M., and Eliopoulos, D. G. (2005), Distribution of rhenium in molybdenite from porphyry $\mathrm{Cu}-\mathrm{Mo}$ and $\mathrm{Mo}-$ $\mathrm{Cu}$ deposits of Russia (Siberia) and Mongolia, Ore Geology Reviews, 26: 91-113. $[78,151]$ 
Boumans, P. W. J. M. (1968), Atomic partition functions in spectrochemical analysis, Spectrochimica Acta, 23B: 559-566. [128]

Campbell-Miller, M. D. and Simard, B. (1996), First ionization potentials of tungsten and rhenium by mass-selected double-resonance ionization spectroscopy, Journal of the Optical Society of America B, 10B: 2115-2120. [126, 134, 163]

Capitant, M., Francotte, J., Picot, P., and Troly, G. (1963), Hautes teneurs de rhénium dans une molybdénite de Kipushi, Comptes Rendus de l'Académie des Sciences, 257: 3443-3444. [75, 150]

Cohen, A. S., Coe, A. L., Bartlett, J. M., and Hawkesworth, C. J. (1999), Precise Re-Os ages of organic-rich mudrocks and the Os isotopic composition of Jurassic seawater, Earth and Planetary Science Letters, 167: 159-173. [76, 78, 150, 151]

Collerson, K. D., Kamber, B. Z., and Schoenberg, R. (2002), Applications of accurate, high-precision $\mathrm{Pb}$ isotope ratio measurement by multi-collector ICP-MS, Chemical Geology, 188: 65-83. [133]

Colodner, D., Sachs, J., Ravizza, G., Turekian, K., Edmond, J., and Boyle, E. (1993), The geochemical cycle of rhenium: a reconnaissance, Earth and Planetary Science Letters, 117: 205-221. [15, 16, 21, 22, 23, 24, 26, 65, 69, 70, 71, 76, 79, 86, 87, 91, $92,94,95,96,102,150,151,197,198]$

Craddock, P. R., Rouxel, O. J., Ball, L. A., and Bach, W. (2008), Sulfur isotope measurements of sulfate and sulfide by high-resolution MC-ICP-MS, Chemical Geology: 102-113. [146]

Creaser, R. A., Papanastassiou, D. A., and Wasserburg, G. J. (1991), Negative thermal ion mass spectrometry of osmium, rhenium, and iridium, Geochimica et Cosmochimica Acta, 55: 397-401. [127, 130] 
Creaser, R. A., Sannigrahi, P., Chacko, T., and Selby, D. (2002), Further evaluation of the Re-Os geochronometer in organic-rich sedimentary rocks: A test of hydrocarbon maturation effects in the Exshaw Formation, Western Canada Sedimentary Basin, Geochimica et Cosmochimica Acta, 66: 3441-3452. [78, 150, 151, 181, 186]

Dalai, T. K., Singh, S. K., Trivedi, J. R., and Krishnaswami, S. (2002), Dissolved rhenium in the Yamuna River System and the Ganga in the Himalaya: role of black shale weathering on the budgets of Re, Os, and $\mathrm{U}$ in rivers and $\mathrm{CO}_{2}$ in the atmosphere, Geochimica et Cosmochimica Acta, 66: 29-43. [76, 87, 95, 150, 151]

de Galan, L., Smith, R., and Winefordner, J. D. (1968), The electronic partition functions of atoms and ions between $1500 \mathrm{~K}$ and $7000 \mathrm{~K}$, Spectrochimica Acta, 23B: 521-525. [128]

Dąbek, J. and Stanislaw, H. (2007), Physical foundations of rhenium-osmium method - a review, Chronometria, 27: 23-26. [128]

Dresser, M. J. (1968), The Saha-Langmuir equation and its application, Journal of Applied Physics, 39: 338-339. [128]

Ellis, A. S., Johnson, T. M., and Bullen, T. D. (2002), Chromium isotopes and the fate of hexavalent chromium in the environment, Science, 295: 2060-2062. [129]

Esser, B. K. and Turekian, K. K. (1993), The osmium isotopic composition of the continental crust, Geochimica et Cosmochimica Acta, 57: 3093-3104. [73, 149, 151, $176,184]$

Fleischer, M. (1959), The geochemistry of rhenium, with special reference to its occurrence in molybdenite, Economic Geology, 55: 607-609. [75, 78, 150, 151]

Fleischer, M. (1960), The geochemistry of rhenium-addendum, Economic Geology, 55: 607-609. [78, 150] 
Galer, S. J. G. (1999), Optimal double and triple spiking for high precision lead isotopic measurement, Chemical Geology, 157: 255-274. [132]

Gramlich, J. W., Murphy, T. J., Garner, E. L., and Shields, W. R. (1973), Absolute isotopic abundance ratio and atomic weight of a reference sample of rhenium, Journal of Research of the National Bureau of Standards - A. Physics and Chemistry, 77A: 691-698. [126, 127, 128, 129, 137, 138, 140, 143, 146, 178]

Gregoire, D. C. (1990), Sample introduction techniques for the determination of osmium isotope ratios by inductively coupled plasma mass spectrometry, Analytical Chemistry, 62: 141-146. [155]

Habfast, K. (1998), Fractionation correction and multiple collectors in thermal ionization isotope ratio mass spectrometry, International Journal of Mass Spectrometry, 176: 133-148. [135]

Halliday, A. N., Lee, D.-C., Christensen, J. N., Walder, A. J., Freedman, P. A., Jones, C. E., Hall, C. M., Yi, W., and Teagle, D. (1995), Recent developments in inductively coupled plasma magnetic sector multiple collector mass spectrometry, International Journal of Mass Spectrometry and Ion Processes, 146/147: 21-33. $[127,146]$

Harper, C. L. J. and Jacobsen, S. B. (1996), Evidence for ${ }^{182} \mathrm{Hf}$ in the early solar system and constraints on the timescale of terrestrial and core formation, Geochimica et Cosmochimica Acta, 60: 1131-1153. [127]

Hart, S. R. and Zindler, A. (1989), Isotopic fractionation laws: A test using calcium, International Journal of Mass Spectrometry and Ion Processes, 89: 287-301. [129, $135,138,142]$

Hassler, D. R., Peucker-Ehrenbrink, B., and Ravizza, G. E. (2000), Rapid determination of Os isotopic composition by sparging $\mathrm{OsO}_{4}$ into a magnetic-sector ICP-MS, Chemical Geology, 166: 1-14. [155] 
Hauri, E. H. and Hart, S. R. (1997), Rhenium abundances and systematics in oceanic basalts, Chemical Geology, 139: 185-205. [73, 149, 176, 184]

Hirata, T. (1996), Lead isotopic analyses of NIST standard reference materials using multiple collector inductively coupled plasma mass spectrometry coupled with a modified external correction method for mass discrimination effect, Analyst, 121: 1407-1411. [134, 135, 138, 140, 142, 159, 163]

Houk, R. S. (1986), Mass spectrometry of inductively coupled plasmas, Analytical Chemistry, 58: 97A-105A. [127, 128, 134]

Houk, R. S., Fassel, V. A., Flesch, G. D., Svec, H. J., Gray, A. L., and Taylor, C. E. (1980), Inductively coupled argon plasma for mass spectrometric determination of trace elements, Analytical Chemistry, 52: 2283-2289. [127]

Irisawa, K. and Hirata, T. (2006), Tungsten isotopic analysis on six geochemical reference materials using multiple collector-icp-mass spectrometry coupled with a rhenium external-correction technique, Journal of Analytical Atomic Spectrometry, 21: 1387-1395. [134, 138, 143, 146, 150]

Kane, J. S., Arbogast, B., and Leventhal, J. (1990), Characterization of Devonian Ohio Shale SDO-1 as a USGS geochemical reference sample, Geostandards Newsletter, 14: 169-196. [129]

Kawano, H. (2006), Mean work functions effective for negative-ionic, electronic and positive ionic emissions from polycrystalline surfaces, Applied Surface Science, 252: 5233-5242. [128]

Korzhinski, M. A., Tkachenko, S. I., Shmulovich, K. I., Taran, Y. A., and Steinberg, G. S. (1994), Discovery of pure rhenium mineral at Kudriavy volcano, Nature, 369: 51-52. [75, 150, 151] 
Lee, D.-C. and Halliday, A. N. (1995a), Hafnium-tungsten chronometry and the timing of terrestrial core formation, Nature, 378: 771-774. [129, 134, 143]

Lee, D.-C. and Halliday, A. N. (1995b), Precise determinations of the isotopic compositions and atomic weights of molybdenum, tellurium, tin and tungsten using ICP magnetic sector multiple collector mass spectrometry, International Journal of Mass Spectrometry and Ion Processes, 146/147: 35-46. [129]

Longerich, H. P., Fryer, B. J., and Strong, D. F. (1987), Determination of lead isotope ratios by inductively coupled plasma-mass spectrometry (ICP-MS), Spectrochimica Acta, 42B: 39-48. [133]

Luais, B., Telouk, P., and Albarède, F. (1997), Precise and accurate neodymium isotopic measurements by plasma-source mass spectrometry, Geochimica et Cosmochimica Acta, 61: 4847-4854. [138]

Mao, J., Du, A., Seltmann, R., and Yu, Y. (2003), Re-Os ages for the Shamika porphyry Mo deposit and the Lipovy Log rare metal pegmatite, central Urals, Russia, Mineralium Deposita, 38: 251-257. [78, 151]

Mao, J., Wang, Y., Lehmann, B., Yu, Y., Du, A., Mei, Y., Li, Y., Zang, W., Stein, H. J., and Zhou, T. (2006), Molybdenite Re - Os and albite ${ }^{40} \mathrm{Ar} /{ }^{39} \mathrm{Ar}$ dating of $\mathrm{Cu}-\mathrm{Au}-\mathrm{Mo}$ and magnetite porphyry systems in the Yangtze River valley and metallogenic implications, Ore Geology Reviews, 29: 307-324. [78, 151]

Maréchal, C. N., Télouk, P., and Albarède, F. (1999), Precise analysis of copper and zinc isotopic compositions by plasma-source mass spectrometry, Chemical Geology, 156: 251-273. [129, 133, 138, 140, 142, 159, 163]

Markey, R., Stein, H., and Morgan, J. (1998), Highly precise Re-Os dating for molybdenite using alkaline fusion and NTIMS, Talanta, 45: 935-946. [78, 151] 
Markowski, A., Quitté, G., Halliday, A. N., and Kleine, T. (2006), Tungsten isotopic compositions of iron meteorites: chronological constraints vs. cosmogenic effects., Earth and Planetary Science Letters, 242: 1-15. [134, 143]

McCandless, T. E., Ruiz, J., and Campbell, A. R. (1993), Rhenium behavior in molybdenite in hypogene and near surface environments: implications for Re-Os geochronomtery, Geochimica et Cosmochimica Acta, 57: 889-905. [76, 78, 151]

McDonough, W. F. and Sun, S. (1995), The composition of the Earth, Chemical Geology, 120: 223-253. [149]

Miller, M. (1992), Basic concepts in atomic emission spectroscopy, chap. 2, VCH Publishers Inc., 55-108. [128]

Mitchell, R. H., Laflamme, J. H. G., and Cabri, L. J. (1989), Rhenium sulphide from the Coldwell complex, northwestern Ontario, Canada, Mineralogical Magazine, 53: 635-637. [75, 150]

Morachevskii, D. E. and Nechaeva, A. A. (1960), Characteristics of migration of rhenium from molybdenites, Geochemistry, 6: 648-651. [76, 78, 150, 151]

Morris, D. F. C. and Short, E. L. (1966), Minerals of rhenium, Mineralogical Magazine, 35: 871-873. [75, 150]

Nielsen, S. G., Rehkämper, M., Baker, J., and Halliday, A. N. (2004), The precise and accurate determination of thallium isotope compositions and concentrations for water samples by MC-ICPMS, Chemical Geology, 204: 109-124. [129]

Noddack, I. and Noddack, W. (1931), Die Geochemie des Rheniums, Zeitschrift für Physikalische Chemie, 16: 207-244. [74, 78, 149, 151]

Noddack, V. W., Tacke, I., and Berg, V. O. (1925), Zwei neue Elemente der Mangangruppe, Sitzung der physikalisch-mathematischen Klasse, 11: 400-409. [74, 75, 149] 
Peucker-Ehrenbrink, B. and Hannigan, R. E. (2000), Effects of black shale weathering on the mobility of rhenium and platinum group elements, Geology, 28: 475-478. $[17,76,78,150,151,176,179,198]$

Power, M. R., Pirrie, D., Jedwab, J., and Stanley, C. J. (2004), Platinum-group element mineralization in an As-rich magmatic sulphide system, Talnotrie, southwest Scotland, Mineralogical Magazine, 68: 395-411. [75, 150]

Ravizza, G. E. and Turekian, K. K. (1989), Application of the ${ }^{187}$ Re- ${ }^{187}$ Os system to black shale geochronometry, Geochimica et Cosmochimica Acta, 53: 3257-3262. $[15,78,150,151]$

Ravizza, G. E., Turekian, K. K., and Hay, B. J. (1991), The geochemistry of rhenium and osmium in recent sediments from the Black Sea, Geochimica et Cosmochimica Acta, 55: 3741-3752. [15, 75, 150, 151, 198]

Rayleigh, J. W. S. (1902), On the distillation of binary mixtures, Philosophical Magazine, 4: 521-537. [135, 187]

Ripperger, S. and Rehkämper, M. (2007), Precise determination of cadmium isotope fractionation in seawater by double spike MC-ICP-MS, Geochimica et Cosmochimica Acta, 71: 631-642. [129]

Roe, J. E., Anbar, A. D., and Barling, J. (2003), Nonbiological fractionation of Fe isotopes: evidence of an equilibrium isotope effect, Chemical Geology, 195: 69-85. $[150]$

Rosman, K. J. R. and Taylor, P. D. P. (1998), Isotopic compositions of the elements 1997, Pure and Applied Chemistry, 70: 217-235. [27, 126, 135]

Russ, G. P., Bazan, J. M., and Date, A. R. (1987), Osmium isotopic ratio measurements by inductively coupled plasma mass spectrometry, Analytical Chemistry, 59: 984-989. [155] 
Russel, W. A., Papanastassiou, D. A., and Tombrello, T. A. (1978), Ca isotope fractionation on the Earth and other solar system materials, Geochimica et Cosmochimica Acta, 42: 1075-1090. [129, 132, 138, 142, 150]

Selby, D. and Creaser, R. A. (2001), Re-Os geochronology and systematics in molybdenite from the Endako porphyry molybdenum deposit, British Columbia, Canada, Economic Geology, 96: 197-204. [78, 151]

Selby, D., Creaser, R. A., Stein, H. J., Markey, R. J., and Hannah, J. L. (2007), Assessment of the ${ }^{187}$ Re decay constant by cross calibration of Re-Os molybdenite and $\mathrm{U}-\mathrm{Pb}$ zircon chronometers in magmatic ore systems, Geochimica et Cosmochimica Acta, 71: 1999-2013. [78, 153]

Suzuki, K., Miyata, Y., and Kanazawa, N. (2004), Precise Re isotope ratio measurements by negative thermal ionization mass spectrometry (NTI-MS) using total evaporation technique, International Journal of Mass Spectrometry, 235: 97-101. $[127]$

Tarkian, M., Housley, R. M., Volborth, A., Greis, O., and Moh, G. (1991), Unnamed Re-Mo-Cu sulfide from the Stillwater complex, and crystal chemistry of its synthetic equivalent spinel type $(\mathrm{Cu}, \mathrm{Fe})(\mathrm{Re}, \mathrm{Mo})_{4} \mathrm{~S}_{8}$, European Journal of Mineralogy, 3 : 977-982. [150]

Taylor, P. D. P., de Bièvre, P., Walder, A. J., and Entwistle, A. (1995), Validation of the analytical linearity and mass discrimination correction model exhibited by a multiple collector inductively coupled plasma mass spectrometer by means of a set of synthetic uranium mixtures, Journal of Analytical Atomic Spectrometry, 10: 395-398. [138]

Terada, K., Osaki, S., Ishihara, S., and Kiba, T. (1971), Distribution of rhenium in molybdenites from Japan, Geochemical Journal, 4: 123-141. [78, 151] 
Vais, V., Li, C., and Cornett, J. (2004), Preventing uranium hydride formation in standard uranium samples for determination of ${ }^{239} \mathrm{Pu}$ by ICP-MS, Journal of Analytic Atomic Spectrometry, 19: 1281-1283. [148]

Vance, D. and Thirlwall, M. (2002), An assessment of mass discrimination in MCICPMS using Nd isotopes, Chemical Geology, 185: 227-240. [135, 138, 142]

Völkening, J., Köppe, M., and Heumann, K. G. (1991a), Tungsten isotope ratio determinations by negative thermal ionization mass spectrometry, International Journal of Mass Spectrometry and Ion Processes, 107: 361-368. [134, 135, 137, $140,143,146,148,162]$

Völkening, J., Walczyk, T., and Heumann, K. G. (1991b), Osmium isotope ratio determinations by negative thermal ionization mass spectrometry, International Journal of Mass Spectrometry and Ion Processes, 105: 147-159. [138]

Walder, A. J. and Freedman, P. A. (1992), Isotopic ratio measurement using a double focusing magnetic sector mass analyzer with an inductively coupled plasma as an ion source, Journal of Analytic Atomic Spectrometry, 7: 571-575. [127, 132, 134, $135]$

Walder, A. J., Platzner, I., and Freedman, P. A. (1993), Isotope ratio measurement of lead, neodymium and neodymium-samarium mixtures, hafnium and hafniumlutetium mixtures with a double focusing multiple collector inductively coupled plasma mass spectrometer, Journal of Analytical Atomic Spectrometry, 8: 19-23. $[127,132,133,134,135]$

White, W. M., Albarède, F., and Télouk, P. (2000), High-precision analysis of Pb isotope ratios by multi-collector ICP-MS, Chemical Geology, 167: 257-270. [133, $138]$ 


\section{Chapter 4}

\section{Rhenium isotopic variation across a black shale weathering profile}

\subsection{Introduction}

The sedimentary organic carbon $\left(\mathrm{C}_{\text {org }}\right)$ or kerogen reservoir $\left(\sim 1.5 \times 10^{19} \mathrm{~kg}, 1.25\right.$ $\left.\times 10^{18} \mathrm{~mol}\right)$ is, next to sedimentary inorganic carbon $\left(\mathrm{C}_{\text {carb }}\right)$, the largest near-surface $\mathrm{C}$ reservoir on Earth $\left(\mathrm{C}_{\text {carb }}: \mathrm{C}_{\text {org }} \sim 1\right.$ 1:4, Hedges And KeIL, 1995). Along with the weathering of $\mathrm{Ca}$ - and $\mathrm{Mg}$-silicates, the burial of $\mathrm{C}_{\text {org }}$ removes $\mathrm{CO}_{2}$ from the atmosphere on long time-scales. Black shales represent an organic-rich kerogen end member as they contain weight percent quantities of $\mathrm{C}_{\mathrm{org}}$. Though the marine environments thought to result in the deposition of black shale lithologies are currently rare (accounting for only $\sim 0.3 \%$ of modern ocean area; MORFORD AND EMERSON, 1999; Algeo, 2004), their widespread occurrence at specific intervals over the Precambrian and Phanerozoic are thought to denote oxygen deficiencies in the geologic past (e.g. Arnold Et AL., 2004; PeArCe ET AL., 2008), and suggest that black shales may have been quantitatively important $\mathrm{C}_{\text {org }}$ sinks.

Black shales also show significant enrichments of elements other than $\mathrm{C}_{\text {org }}$ that make them important in the cycling of trace metals such as Mo, Re, and U. Studies 
of such metals in black shales have been used to reconstruct local and global redox conditions (Crusius et Al., 1996; Arnold et Al., 2004; Algeo and Lyons, 2006).

In particular, Re is reductively-enriched in black shales to a higher degree than any other metal (CRUSiUs ET AL., 1996), and when re-exposed to oxidizing conditions, Re is very effectively mobilized (Colodner et AL., 1992; Crusius AND Thomson, 2000; Peucker-Ehrenbrink and Hannigan, 2000; Jaffe et al., 2002; Pierson-Wickmann et AL., 2003) as it does not sorb to Fe- or Mn-oxides (KoIDE ET AL., 1986). The severity of its response to such redox transitions, and its extremely low abundance in most crustal rocks (EsSER AND TUREKIAN, 1993; Hauri and Hart, 1997; McLennan, 2001; Sun et al., 2003), makes Re an ideal recorder of past redox changes.

The combination of observed $\delta^{98 / 95} \mathrm{Mo}$ and $\delta^{238} \mathrm{U}$ fractionation between modern oxidized and reduced depositional environments (SIEBERT ET AL., 2003; WeYer ET AL., 2008) with observed natural isotopic fractionation for Re (Chapter 3) suggests that $\delta^{187}$ Re analyses might be successfully applied to studies of ancient and modern redox processes. To evaluate this possibility, this study examines Re isotopic fractionation in redox variable environments using a modern black shale weathering profile.

\subsection{Materials and methods}

\subsubsection{Sampling}

Samples were obtained from an outcrop of Devonian New Albany Shale (NAS) near Clay City, KY (Fig 4-1). Road construction in 1960 exposed a buff-coloured weathering rind around a darker, less-weathered core. Strata are nearly horizontal allowing lithologic control while sampling across the weathering profile. Due to these features, weathering studies of $\mathrm{C}_{\text {org }}$ and trace metals have been conducted at this site (PETSCH 
ET AL., 2000, 2001a,b; JAFFe ET AL., 2002; TutTle ET AL., 2003).

The weathering profile was sampled horizontally across a single stratigraphic horizon (Fig 4-2 A), with the degree of weathering decreasing with increasing depth from the edge of the profile. The deepest samples $(>10 \mathrm{~m})$ exhibit large pyrite crystals attesting to the lack of $\mathrm{O}_{2}$ penetration.

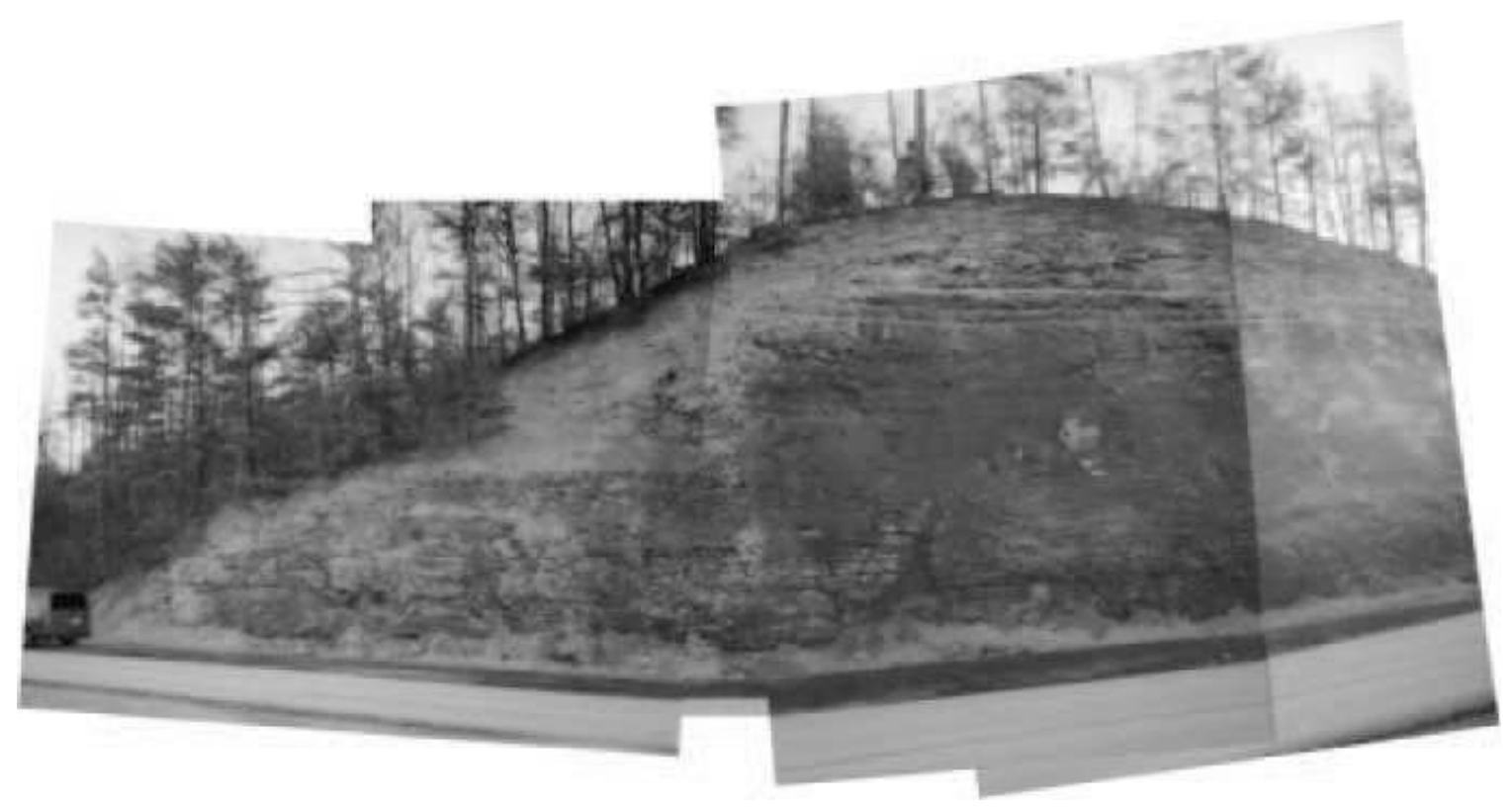

Figure 4-1: A photo mosaic of the New Albany Shale weathering profile near Clay City, Kentucky, USA.

\subsubsection{Elemental analyses}

Concentrations of $\mathrm{C}_{\text {org }}$ and Re were previously published in JAFFE ET AL. (2002). Concentrations of $\mathrm{C}_{\text {org }}$ were determined using a PE 2400 CHN Elemental Analyzer (WHOI), while Re analyses were done by isotope-dilution ICPMS in the WHOI Plasma Mass Spectrometry Facility. Concentrations of Fe, Mo, and U were obtained from the Service d'Analyse des Roches et des Minéraux (SARM) de la Centre Nationale de la Recherche Scientifique (CNRS) in Vandoeuvre-lès-Nancy, France, using ICP-AES (Fe) and ICP-MS (Mo, U). Uncertainties of Fe, Mo, and U are all $<5 \%$. 


\subsection{3 $\quad \delta^{187} \operatorname{Re}$ analyses}

The development of methods for the stable isotope analysis of Re is described in detail in Chapter 3, so only a brief summary is provided here. Sample powders are ashed for $12 \mathrm{hr}$ at $450^{\circ} \mathrm{C}$ to destroy any organics, and then subjected to a series of strong acid leaches (concentrated $\mathrm{HCl}$, inverse aqua regia, concentrated $\mathrm{HNO}_{3}$ ) that liberate more than $80 \%$ of Re. Rhenium is purified by column chromatography, and taken up in $5 \%$ $\mathrm{HNO}_{3}$, doped with tungsten (W) and analyzed by mass spectrometry, using a quartz spray chamber and X-cones. Ion beam intensities of $\mathrm{W}$ and Re are measured using Faraday cups, and ${ }^{189} \mathrm{Os}$ is monitored using the axial secondary electron multiplier (SEM) to provide a correction for isobaric ${ }^{187}$ Os. Instrumental mass fractionation of measured ${ }^{187} \mathrm{Re} /{ }^{185} \mathrm{Re}$ is corrected with ${ }^{186} \mathrm{~W} /{ }^{184} \mathrm{~W}$ according to an exponential law in combination with standard-bracketing. Reproducibility of standard solutions at the analyte levels used for this study (35 ng $\mathrm{W} \mathrm{g}^{-1}, 10 \mathrm{ng} \mathrm{Re}^{-1}$ ) are $\pm 0.1 \%$. Data are expressed in $\%$ using $\delta$ notation,

$$
\delta^{187} R e=\left[\left(\frac{\frac{187}{185} \operatorname{Re} s m p l}{\frac{187 R e}{185} \text { Re } M 989_{\text {true }}}\right)-1\right] \times 1,000
$$

where NIST SRM 989 serves as a reference standard with ${ }^{187} \mathrm{Re} /{ }^{185} \mathrm{Re}$ of 1.6740 (Gramlich ET AL., 1973). The Os isotopic composition $\left({ }^{187} \mathrm{Os} /{ }^{188} \mathrm{Os}\right)$ of these samples has been previously published (JAFFE ET AL., 2002), allowing comparison of Os-corrected and Os-uncorrected $\delta^{187}$ Re values. The largest change imposed by ${ }^{187} \mathrm{Os}$ correction was $0.04 \%$, indicating that further Re purification via sparging is unnecessary (See Chapter 3). 


\begin{tabular}{lccccccc}
\hline Sample & $\begin{array}{c}\text { Distance to } \\
\text { soil horizon, } \mathrm{m}\end{array}$ & $\begin{array}{c}\mathrm{C}_{\text {org, }}{ }^{*} \\
\text { wt. } \%\end{array}$ & $\begin{array}{c}\mathrm{Fe}, \\
\text { wt. \% }\end{array}$ & $\begin{array}{c}\mathrm{Re}^{*} \\
\mathrm{ngg}^{-1}\end{array}$ & $\begin{array}{c}\mathrm{Mo}, \\
\mu \mathrm{gg}^{-1}\end{array}$ & $\begin{array}{c}\mathrm{U}, \\
\mu \mathrm{gg}^{-1}\end{array}$ & $\begin{array}{c}\delta^{187} \mathrm{Re}, \\
\% 0\end{array}$ \\
\hline NAS0 & 0 & 1.8 & 4.9 & 0.89 & 92 & 24 & -0.51 \\
NAS3 & 0.91 & 1.5 & 5.7 & 0.85 & 126 & 30 & -0.59 \\
NAS8 & 2.44 & 4.3 & 3.0 & 18 & 69.4 & 21 & -0.32 \\
NAS14 & 4.27 & 7.3 & 2.7 & 89 & 82.1 & 25 & -0.25 \\
NAS20 & 6.10 & 6.0 & 3.0 & 116 & 73.2 & 17 & -0.24 \\
NAS26 & 7.92 & 7.1 & 3.4 & 75 & 83.7 & 20 & -0.32 \\
NAS36 & 10.97 & 7.8 & 3.3 & 81 & 82.6 & 19 & -0.34 \\
NAS46 & 14.02 & 6.8 & 3.2 & 80. & 74.2 & 39 & -0.22 \\
\hline
\end{tabular}

* Concentrations of Corg and Re are from JAFFE ET AL. (2002).

Table 4.1: Element concentration and $\delta^{187}$ Re data from the NAS weathering profile, near Clay City, KY.

\subsection{Results and Discussion}

\subsubsection{Elemental variability}

The development of a redox gradient, visible as a weathering rind over an unweathered core (Fig 4-1), is represented geochemically by significant decreases in $\mathrm{C}_{\text {org }}$ (Fig 4-2 B), N, and pyritic sulphur (JAFFE ET AL., 2002). The transition to an oxic weathering environment is seen in a $75 \%$ decrease in $\mathrm{C}_{\text {org }}$ coincident with elevated iron concentrations at the edge of the outcrop.

Also shown in in Figure 4-2 are concentration profiles for Re, Mo, and U. These metals, considered to have somewhat similar redox behaviors, show significantly different patterns across the weathering profile. The Mo and U profiles are similar to that of $\mathrm{Fe}$, in that they show enrichment in the more oxidising portion of the weathering horizon. This is likely a secondary enrichment from sorption onto Fe/Mn oxyhydroxides (Koide Et AL., 1986; Siebert ET AL., 2003). In contrast, Re exhibits near total loss in the oxic part of the profile. Rhenium mobility and a lack of oxyhydroxide sorption have been noted for weathering shales before (PEUCKER-EHRENBRINK And Hannigan, 2000; Pierson-Wickmann et AL., 2003). 

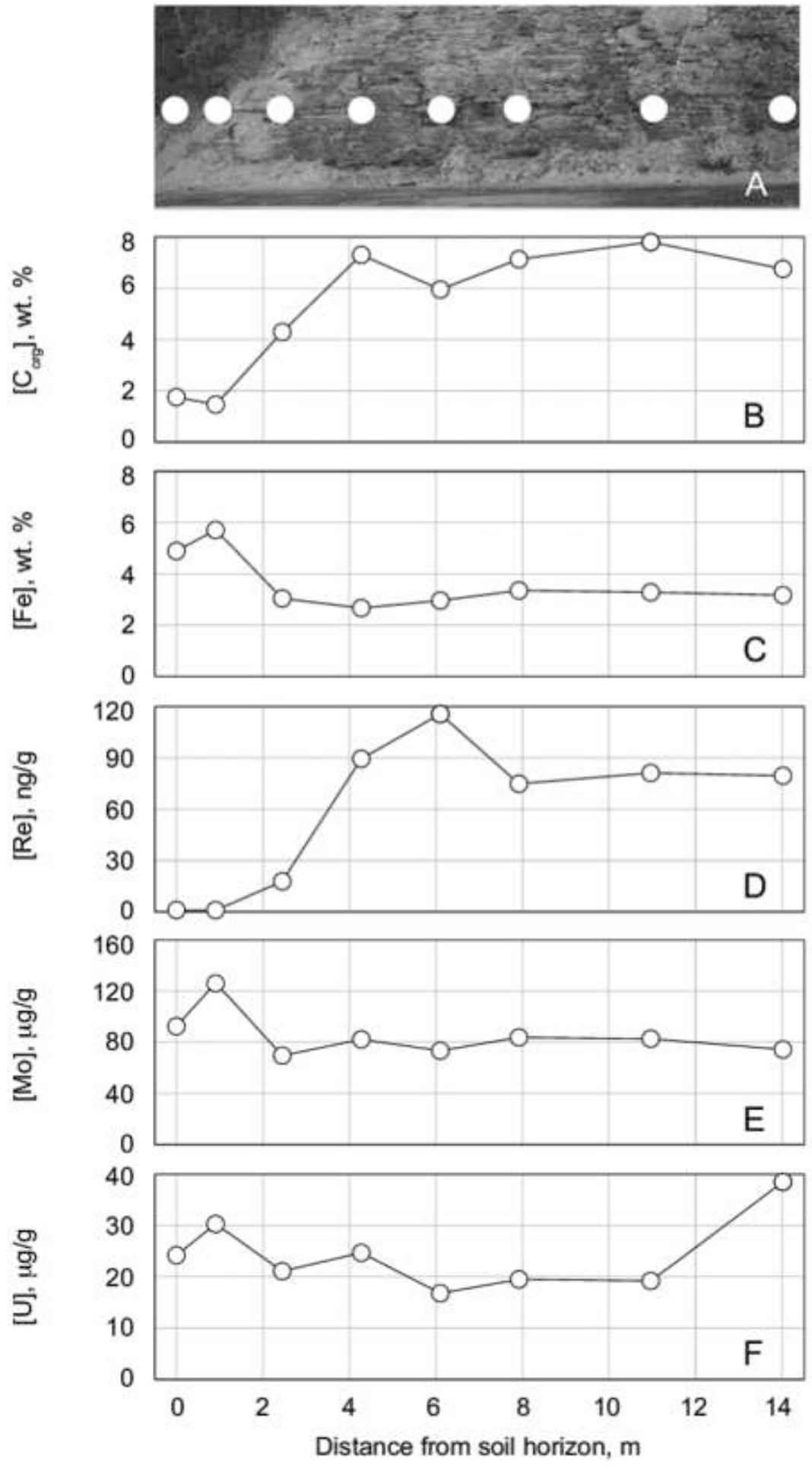

Figure 4-2: Full caption on page 181. 
Figure 4-2: Elemental variation across the New Albany Shale weathering profile.

A: Close-up of sample locations for the NAS weathering profile.

$\mathrm{B}$ : Variation in $\mathrm{C}_{\text {org }}$ : note significant loss in heavily weathered portion.

C: Variation in Fe: note apparent enrichment in heavily weathered portion.

D: Variation in Re: note almost complete loss in heavily weathered portion.

E: Variation in Mo: note apparent enrichment in heavily weathered portion.

F: Variation in U: note apparent enrichment in heavily weathered portion.

\subsubsection{Re isotopic variability}

Rhenium isotopic variation across the New Albany Shale weathering profile near Clay City, Kentucky is shown in Fig 4-3A. The $\delta^{187}$ Re data follow a pattern of fractionation that appears to mimic that of Re concentration, with isotopically heavier values in the "deeper" portion of the profile and isotopically lighter Re in the "shallower," more intensely weathered portion. The coincidence of isotopically distinct Re with changing $\mathrm{C}_{\text {org }}$ and $\mathrm{Re}$ concentrations indicates that this isotopic variability is the result of the oxic weathering process.

All Re isotopic data for the weathering profile are less than 0\%. In contrast, data for four commercially available Re standards have $\delta^{187}$ Re values of $0 \%$ or greater. Total Re isotopic variability seen thus far is $\sim 1 \%$.

In attempting to account for Re isotope variability in an environment showing significant Re mobility, we evaluate two possible Re fractionation models: twocomponent mixing, and Rayleigh fractionation.

\section{Two-component (binary) mixing}

Rhenium mobility is suggested not only by significant abundance fluctuations but also by consideration of the ${ }^{187} \mathrm{Re}^{-187}$ Os decay systematics. Figure 4-4A shows the ${ }^{187} \mathrm{Re} /{ }^{188} \mathrm{Os}$ and ${ }^{187} \mathrm{Os} /{ }^{188}$ Os data for the profile samples, originally published in

JAFFE ET AL. (2002). Also shown is a reference isochron (359 Ma, ${ }^{187} \mathrm{Os} /{ }^{188} \mathrm{Os}_{\text {initial }}$ $=0.59$ ) for samples of the NAS-correlative Exshaw Formation (CREASER ET AL., 

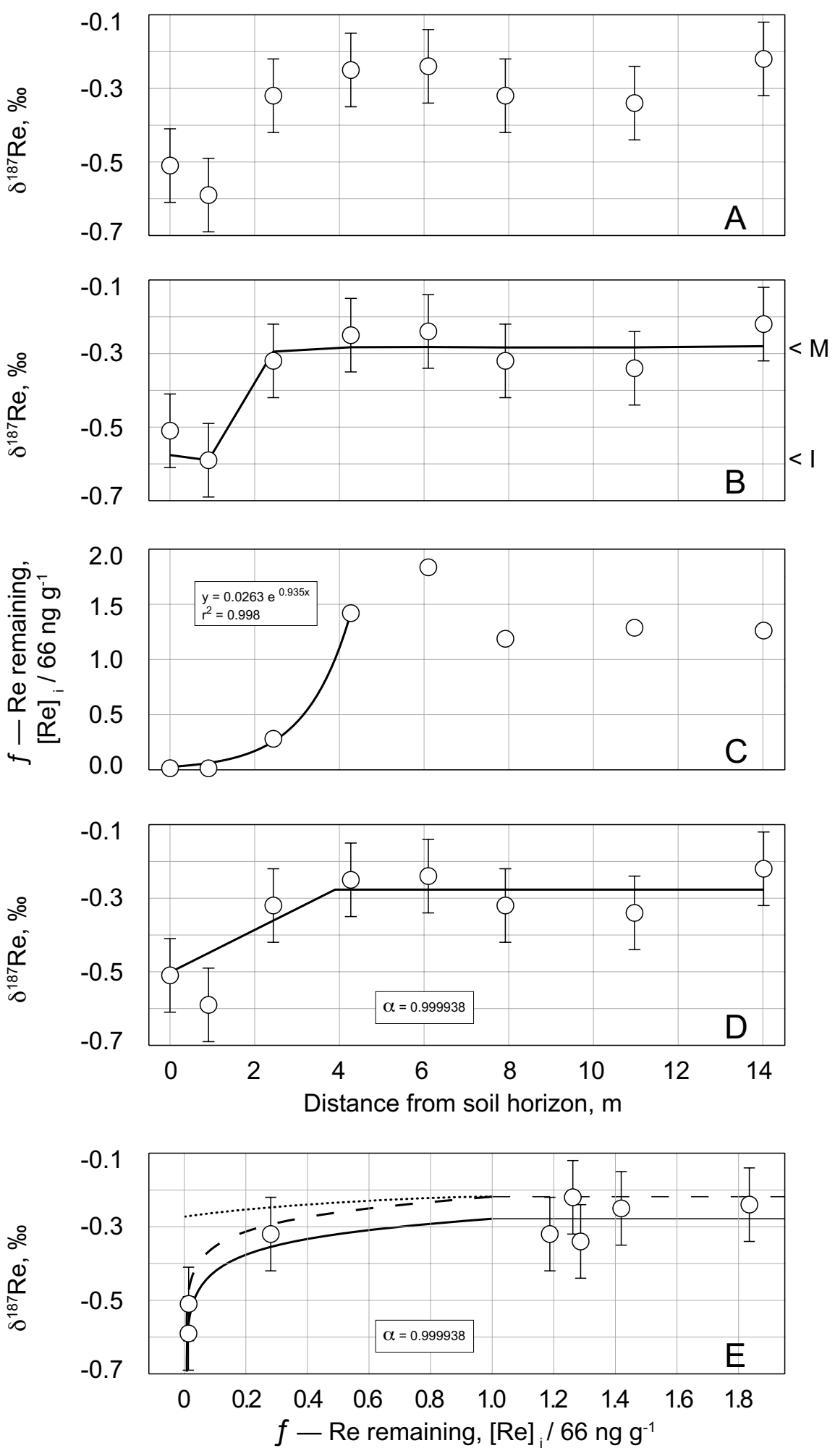

Figure 4-3: See full caption on page 183. 
Figure 4-3: Re isotopic composition and model results for the Clay City, KY, New Albany Shale weathering profile.

A: $\delta^{187}$ Re vs. depth.

B: Isotope data along with the results of a 2-component mixing model-fit; isotopic compositions of the two components - M and I (mobile and immobile) — are indicated at right (see text for further details).

C: Re concentration data re-expressed as $f$ - the fraction of Re remaining relative to some initial amount estimated at $63 \mathrm{ng} \mathrm{g}^{-1}$. Original Re concentration data is from JAFFE ET AL. (2002) . Also shown is a best-fit linear regression according to an exponential model for samples NAS0, NAS3, NAS8, and NAS14 (see text for details). Values of $f$ below 1 are amenable to Rayleigh modeling.

D: Isotope data along with the results of a Rayleigh fractionation model-fit. Values of $f$ were obtained from the $f$ vs. depth relationship illustrated in C.

E: The same model and isotope data as D now plotted vs. $f$ and not depth. Model fits corresponding to residual Re (solid line), instantaneously mobilized Re (dashed line) and pooled mobilized Re (stippled line) are also shown.

$2002)$, and the best-fit $\left(r^{2}=0.42\right)$ linear regression to the data $\left({ }^{187} \mathrm{Re}^{-187}\right.$ Os date of $18 \mathrm{Ma} ;{ }^{187} \mathrm{Os} /{ }^{188} \mathrm{Os}_{\text {initial }}$ of $\sim 6.1$ ). The failure of the profile data to plot on the reference isochron indicates open system behavior; the large spread in ${ }^{187} \mathrm{Re} /{ }^{188} \mathrm{Os}$ relative to ${ }^{187} \mathrm{Os} /{ }^{188} \mathrm{Os}$ along with the more restricted Os mobility (JAFFE ET AL., 2002), indicates that the open-system behavior is dominated by Re mobility.

The occurrence of Re mobility, therefore, suggests that the observed $\delta^{187}$ Re variation could be the result of mixing between 2 or more isotopically distinct Re reservoirs. That black shales are themselves mixtures of inorganic and organic phases suggests the possibility that they might host different Re reservoirs. This is further supported by occurrence of isotopically heavy and light Re in the respectively $\mathrm{C}_{\text {org }}$-rich and -poor portions of the outcrop.

If the $\delta^{187}$ Re profile is the result of 2 -component mixing, and we hypothesize that one Re component is isotopically light, silicate-hosted, immobile, and present at lower abundances, it is likely well-represented by sample NAS3 $(0.91 \mathrm{~m})$. Sample NAS3 has the lowest concentration of $\mathrm{C}_{\text {org }}$ and is therefore the sample most dominated by silicate material. It has the lowest Re concentration observed, $0.85 \mathrm{ng} \mathrm{g}^{-1}$ (JAFFE 
ET AL., 2002), consistent with crustal Re estimates ranging from 0.2 to $2 \mathrm{ngg}^{-1}$ (Esser and Turekian, 1993; Hauri and Hart, 1997; Peucker-Ehrenbrink And Jahn, 2001; Sun ET AL., 2003). Finally, NAS3 also has the lowest value of $\delta^{187}$ Re. Therefore, the concentration and $\delta^{187}$ Re features of NAS3 shall represent the proposed immobile, silicate-hosted, isotopically light Re component.

Unfortunately, the choice of NAS3 to represent the immobile end-member cannot be duplicated in determining the mobile end-member as other profile samples must represent mixtures of mobile and immobile Re components. Fortunately, the original Re concentrations of the samples can be reconstructed assuming open system behavior that is dominated by Re mobility and by using the deviations of the samples from the reference isochron in ${ }^{187} \mathrm{Re} /{ }^{188} \mathrm{Os}$ (Fig 4-4A). Samples to the left of the reference isochron have lost Re, those to the right have gained Re from weathering of overlying horizons, with the magnitude of the deviation is used to determine the amount of Re lost or gained. If our assumptions are correct, the "primary" Re concentrations, recalculated on an individual basis, should all be consistent. Calculated "primary" Re concentrations are shown in Fig 4-4B (black circles), With a single exception (NAS0) they cluster about a mean of $66 \mathrm{ngg}^{-1}$ with a standard deviation (1 s.d.) of $4.6 \mathrm{ng}$ $\mathrm{g}^{-1}(7 \%)$.

If we assume that NAS3 represents the immobile, silicate-hosted baseline to which varying amounts of the mobile component are added, and that any Re added from overlying strata (see Fig 4-1) has a similar $\delta^{187}$ Re, we can subtract the NAS3 isotopic signature from the other samples to determine the $\delta^{187}$ Re of the mobilized fraction. Again, if our assumptions are valid, the resulting isotopic compositions obtained for the mobile fraction should all be consistent. With one exception (NAS0), the data cluster around a mean of $-0.28 \%$ with a standard deviation of $0.10 \%$ ( 2 s.d.).

Neither the initial Re concentration of the samples nor the $\delta^{187}$ Re of the mobile fraction were determined using data from sample NAS0. The results from this sample are anomalous in both respects, exhibiting a recalculated Re concentration of $33 \mathrm{ng}$ 

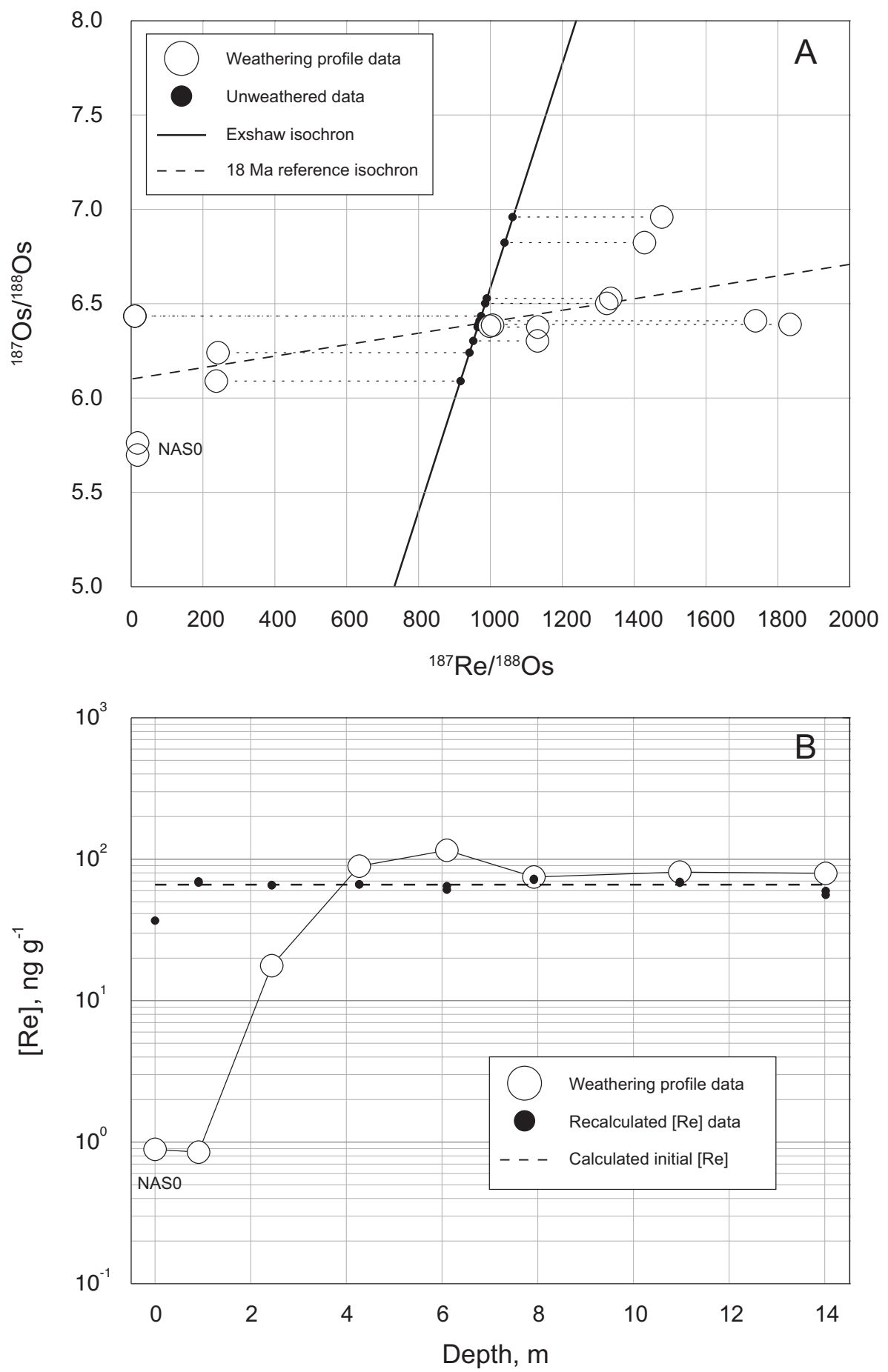

Figure 4-4: See full caption on page 186. 
Figure 4-4: ${ }^{187} \mathrm{Re}-{ }^{187}$ Os weathering profile systematics.

A: ${ }^{187}$ Os $/{ }^{188}$ Os vs. ${ }^{187} \mathrm{Re} /{ }^{188}$ Os for Clay City New Albany Shale weathering outcrop samples after JAFFE ET AL. (2002). Reference isochron data was taken from CREASER ET AL. (2002).

B: Re concentration vs. depth as both actual data and data recalculated with ${ }^{187}{ }^{R e}{ }^{-187}$ Os modeling. The best-estimate initial [Re] of $66 \mathrm{ngg}^{-1}$ (dashed line) was determined by averaging recalculated initial Re concentrations for all samples except NAS0; NAS0 was excluded due to the Os mobility it displays (JAFFE ET AL., 2002).

$\mathrm{g}^{-1}$ and $\delta^{187} \operatorname{Re}$ of $1.27 \%$ for the mobile Re fraction. That these results do not represent the weathering profile as a whole is supported by JAFFE ET AL. (2002) who determined that this sample also displays loss of radiogenic Os.

The results of mixing between consistent amounts of an immobile, isotopically light, silicate-hosted Re component and variable amounts of a mobile, isotopically heavy Re component is shown in Fig 4-3B (solid line). A two-component mixing model accounts quite well for the observed $\delta^{187}$ Re variations.

If two-component mixing appropriately describes the observed $\delta^{187}$ Re variation, then the NAS weathering profile records two isotopically distinct, contemporaneous Re sources. The first is a detrital source of low Re abundance with a $\delta^{187} \operatorname{Re}$ of $\sim$ $-0.59 \%$ (represented by NAS3). The second source dominates the Re inventory, is likely hydrogenous, and is isotopically heavier at $\sim-0.28 \%$. Rhenium is enriched in reducing sedimentary environments on a time-scale of weeks or months and to a greater extent than any other element (CRUsius ET AL., 1996). Given that modern anoxic environments with free $\mathrm{H}_{2} \mathrm{~S}$ are known to record the Mo isotopic composition of modern seawater (Siebert ET AL., 2003; Arnold Et Al., 2004; NeuberT ET AL., 2008), it is plausible that ancient black shales record the metal isotopic composition of contemporaneous seawater. If this is the case, and if seawater Re isotopic composition varies with time, then the much shorter seawater response times $\left(\tau_{\operatorname{Re}}\right)$ reported in Chapter 2 would enable black shales to record $\delta^{187}$ Re variations that are far less pronounced or even invisible to $\delta^{98 / 95} \mathrm{Mo}$. 


\section{Rayleigh fractionation}

Alternatively, the $\delta^{187}$ Re variability across the weathering profile could be the result of Rayleigh fractionation (RAYLEIGH, 1902) — in which the removal process consistently and continually fractionates the isotopically changing Re pool. For the case of Re dissolution from a solid pool, it is described by:

$$
\frac{{ }^{187} R e}{{ }^{185} R e}=\frac{{ }^{187} R e}{{ }^{185} R e} \text { initial } \times f\left(\frac{1}{\alpha}-1\right)
$$

where ${ }^{187} \operatorname{Re} /{ }^{185} \operatorname{Re}$ is the isotopic composition of a given sample, ${ }^{187} \operatorname{Re} /{ }^{185} \mathrm{Re}_{\text {initial }}$ is the initial Re isotopic composition of the samples (calculated at $-0.28 \%$, essentially identical to calculated $\delta^{187}$ Re of the mobile component for the mixing model), $f$ is the fraction of the original Re remaining the Re concentration normalized to the "primary" Re concentration of $66 \mathrm{ng} \mathrm{g}^{-1}$ ) and $\alpha$ is the fractionation factor.

A model for the exponential decrease of $f$ across the first four samples $(4.27 \mathrm{~m})$ of the weathering profile is presented in Fig 4-3C. Though the interval shows progressive Re loss across the first 5 samples $(6.10 \mathrm{~m})$, the samples at $4.27 \mathrm{~m}$ and $6.10 \mathrm{~m}$ have Re concentrations higher than the reconstructed "primary" concentration of $66 \mathrm{ngg}^{-1}$; the drop in concentration from 6.10 to $4.27 \mathrm{~m}$ might not represent actual loss of Re but merely a lesser amount of Re gain.

The model of Re loss presented in Fig 4-3 C predicts a decrease in $f$ below the calculated "primary" value at distances from the soil horizon shorter than $\sim 3.8 \mathrm{~m}$. A Rayleigh fractionation model is fit to the first 4 points of the profile (assuming an $f$ value of 1 for NAS14 at $4.27 \mathrm{~m}$ ) to obtain a best-fit fractionation factor $(\alpha)$ of 0.999938. This fractionation factor and the modeled $f$ values are then used to the calculate expected values of $\delta^{187}$ Re for the heavily-weathered portion of the profile presented in Fig 4-3D (solid line). Samples from deeper in the outcrop all show evidence of Re gain and so have their $\delta^{187}$ Re values held at $-0.28 \%$. The same model and isotopic data, plotted against $f$ (note different scaling) are presented in Fig 4- 
$3 \mathrm{E} ; \delta^{187}$ Re values for co-evolving instantaneous (dashed line) and pooled (stippled line) dissolved Re are also shown. Like two-component mixing, a Rayleigh model of isotopic fractionation during oxic Re weathering also describes the isotopic variability seen across the weathering profile fairly well.

The Rayleigh model, with its $\alpha$ value of 0.99938 , predicts that instantaneously dissolved Re will be $0.62 \%$ heavier than the solid Re from which it is sourced. Rayleigh fractionation of Fe isotopes for regional basaltic weathering across the Maui Climate Gradient (Thompson ET AL., 2007) shows progressively lighter remaining Fe due to increasing reductive loss. Our outcrop-scale study shows increasingly lighter Re with progressive oxidative loss. The hypothesis of instantaneously mobilized Re that is isotopically heavier than total pool is also supported by the evaluation of chromatographic Re fractionation (Chapter 3), in which initially eluded Re fractions were isotopically heavy.

Figure 4-3E displays the $\delta^{187}$ Re of pooled dissolved Re with progressive Rayleigh fractionation (stippled line). In this weathering horizon, it appears as though loss of labile Re is complete, and dissolved Re has an isotopic composition identical to that of unweathered shale. If Re loss is incomplete at the outcrop or regional scale, however, the $\delta^{187}$ Re of the dissolved portion will represent the extent of Re loss and, due to their similar appearance in the weathering profile (Fig 4-2B, 4-2D), the extent of $\mathrm{C}_{\text {org }}$ loss as well. Metal isotope ratios might therefore be useful in distinguishing between ancient and merely old ( $>35,000$ a) remineralized $\mathrm{C}_{\text {org }}$ pools, something to which radiocarbon is insensitive.

\subsubsection{Testing models of Re fractionation}

Because both binary-mixing and Rayleigh fractionation describe the observed $\delta^{187} \operatorname{Re}$ variability quite well, further work is required to distinguish the appropriate fractionation model. Two-component mixing may be evaluated by the isolation and characterization of an organically-hosted Re fraction. Previous research indicates that 
large proportions of Re are solvent-extractable from organic-rich sediments (MILLER, 2004). Figure 4-5 demonstrates the solvent-extraction behavior for several metals from a sample of the Exshaw shale. The metals V, Ni, and Re all extract in wt. $\%$ quantities. Vanadium and $\mathrm{Ni}$ in black shales are often found as organometallic species called porphyrins (QUIRKE, 1987). The similar extraction behavior of Re, V, and $\mathrm{Ni}$ suggests that Re too may form organometallic compounds. In contrast, other reductively-enriched metals such as Mo and $\mathrm{U}$ are not solvent extractable and so are unlikely to bond directly with the organic matter. The presence of organically-hosted Re supports the idea of distinct metal pools within a black shale, and its extractability provides the means of isolating it for $\delta^{187} \operatorname{Re}$ characterization.

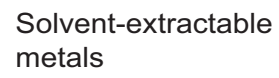

$\mathrm{V}: 4 \%$

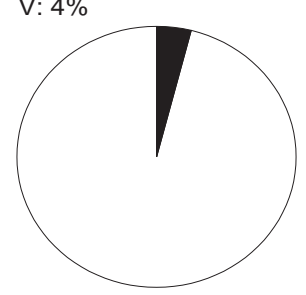

Ni: $7 \%$

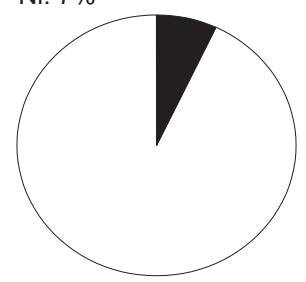

Re: $24 \%$

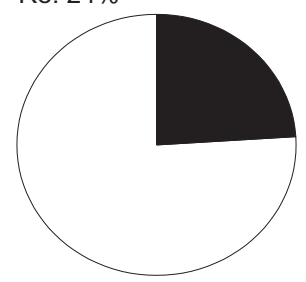

Non extractable

metals

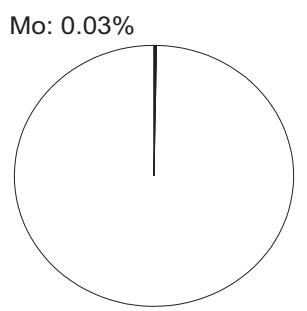

$\mathrm{U}: \sim 0 \%$ (not detectable)

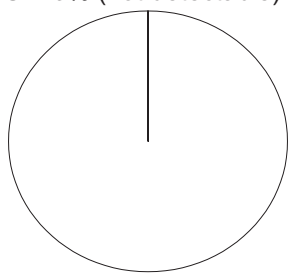

Figure 4-5: Degrees of metal extraction from a sample of the NAS-correlative Exshaw shale, Western Canada Sedimentary Basin. 
Evaluation of Rayleigh fractionation could in-turn be tested with a progressive weathering experiment. FALK ET AL. (2006) have shown that a laboratory-based black shale weathering experiment is able to mobilize sufficient quantities of metals from a few hundred grams of material on a time-scale of weeks. In particular, FALK ET AL. (2006) saw significant mobility of Cd, Ni, and Zn. An examination of the weathering profile concentrations of these metals (not shown) does not show the degree of loss seen for Re, so a similar experiment using New Albany Shale suggests that sufficient Re for $\delta^{187}$ Re analysis would be mobilized on the order of a few months. These progressive weathering products could then be used to test the Rayleigh fractionation model under more controlled conditions.

\subsection{Conclusions}

This study reports the first observations of Re isotope fractionation in a natural setting. Similar patterns of $\mathrm{C}_{\text {org }}$ and Re loss associated with a decrease in $\delta^{187} \mathrm{Re}$ indicate that the isotopic composition of Re responds to changes in the local redox environment. The $\delta^{187}$ Re profile can be interpreted as either two-component mixing, or Rayleigh fractionation during Re weathering. Though sufficient data do not currently exist to allow us to discriminate between these models, both are testable and in either case provide useful information about potential applications of this new isotope system. Two-component mixing suggests that black shales may record contemporaneous seawater $\delta^{187}$ Re while Rayleigh fractionation may allow for the identification of ancient $\mathrm{C}_{\text {org }}$ weathering at regional scales.

\subsection{Acknowledgements}

We would like to acknowledge financial support from NSF-EAR grant 0519387 and from the WHOI Academic Programs Office as well as support for the WHOI Plasma 
Mass Spectrometry Facility from NSF-EAR/IF grants 0318137 and 0651366 . We would also like to thank the members of the WHOI Plasma Mass Spectrometry Facility: Scot Birdwhistell, Jerzy Blusztajn, and Dave Schneider. Samples were collected by Steve Petsch (UMass, Amherst) and processed for analysis by Lillie Jaffe. 


\section{Bibliography}

Algeo, T. J. (2004), Can marine anoxic events draw down the trace element inventory of seawater?, Geology, 32: 1057-1060. [15, 91, 92, 175, 199]

Algeo, T. J. and Lyons, T. W. (2006), Mo-total organic carbon in modern anoxic marine environments: Implications for analysis of paleoredox and paleohydrographic conditions, Paleoceanography, 21: 23 pp. [16, 22, 23, 75, 176]

Arnold, G. L., Anbar, A. D., Barling, J., and Lyons, T. W. (2004), Molybdenum isotope evidence for widespread anoxia in mid-Proterozoic oceans, Science, 304: 87-90. [16, 22, 98, 104, 129, 175, 176, 186, 198, 199]

Colodner, D. C., Boyle, E. A., Edmond, J. M., and Thomson, J. (1992), Postdepositional mobiity of platinum, iridium and rhenium in marine sediments, Nature, 358: 402-404. [176]

Creaser, R. A., Sannigrahi, P., Chacko, T., and Selby, D. (2002), Further evaluation of the Re-Os geochronometer in organic-rich sedimentary rocks: A test of hydrocarbon maturation effects in the Exshaw Formation, Western Canada Sedimentary Basin, Geochimica et Cosmochimica Acta, 66: 3441-3452. [78, 150, 151, 181, 186]

Crusius, J., Calvert, S., Pederson, T., and Sage, D. (1996), Rhenium and molybdenum enrichments in sediments as indicators of oxic, suboxic and sulfidic conditions of deposition, Earth and Planetary Science Letters, 145: 65-78. [15, 17, 21, 22, 75, $92,176,186,198]$

Crusius, J. and Thomson, J. (2000), Comparative behavior of authigenic Re, U, and Mo during reoxidation and subsequent long-term burial in marine sediments, Geochimica et Cosmochimica Acta, 64: 2233-2242. [176, 198]

Esser, B. K. and Turekian, K. K. (1993), The osmium isotopic composition of the 
continental crust, Geochimica et Cosmochimica Acta, 57: 3093-3104. [73, 149, 151, $176,184]$

Falk, H., Lavergren, U., and Bergbäck, B. (2006), Metal mobility in Alum Shale from Öland, Sweden, Journal of Geochemical Exploration, 90: 157-165. [190]

Gramlich, J. W., Murphy, T. J., Garner, E. L., and Shields, W. R. (1973), Absolute isotopic abundance ratio and atomic weight of a reference sample of rhenium, Journal of Research of the National Bureau of Standards - A. Physics and Chemistry, 77A: 691-698. [126, 127, 128, 129, 137, 138, 140, 143, 146, 178]

Hauri, E. H. and Hart, S. R. (1997), Rhenium abundances and systematics in oceanic basalts, Chemical Geology, 139: 185-205. [73, 149, 176, 184]

Hedges, J. I. and Keil, R. G. (1995), Sedimentary organic matter preservation: an assessment and speculative synthesis, Marine Chemistry, 49: 81-115. [175]

Jaffe, L. A., Peucker-Ehrenbrink, B., and Petsch, S. T. (2002), Mobility of rhenium, platinum group elements and organic carbon during black shale weathering, Earth and Planetary Science Letters, 198: 339-353. [17, 21, 76, 176, 177, 178, 179, 181, $183,186,198]$

Koide, M., Hodge, V. F., Yang, J. S., Stallard, M., Goldberg, E. G., Calhoun, J., and Bertine, K. K. (1986), Some comparative marine chemistries of rhenium, gold, silver and molybdenum, Applied Geochemistry, 1: 705-714. [17, 75, 76, 88, 176, $179,198,199]$

McLennan, S. M. (2001), Relationships between the trace element composition of sedimentary rocks and upper continental crust, Geochemistry, Geophysics, Geosystems, 2: doi:10.1029/2000GC000109. [71, 74, 77, 79, 176]

Miller, C. A. (2004), Re-Os dating of algal laminites: reduction enrichment of metals 
in the sedimentary environment and new geoporphyrins, Master's thesis, University of Saskatchewan. [76, 189]

Morford, J. L. and Emerson, S. (1999), The geochemistry of redox sensitive trace metals in sediments, Geochimica et Cosmochimica Acta, 63: 1735-1750. [15, 16, $17,21,22,23,75,91,92,96,175,198,200]$

Neubert, N., Nägler, T. F., and Böttcher, M. E. (2008), Sulfidity controls molybdenum isotope fractionation into euxinic sediments: Evidence from the modern Black Sea, Geology, 36: 775-778. [16, 22, 186]

Pearce, C. R., Cohen, A. S., Coe, A. L., and Burton, K. W. (2008), Molybdenum isotope evidence for global ocean anoxia coupled with perturbations to the carbon cycle during the Early Jurassic, Geology, 36: 231-234. [16, 93, 98, 175, 199]

Petsch, S. T., Berner, R. A., and Eglinton, T. I. (2000), A field study of the chemical weathering of ancient sedimentary organic matter, Organic Geochemistry, 31: 475487. [176]

Petsch, S. T., Eglinton, T. I., and Edwards, K. J. (2001a), ${ }^{14}$ C-dead living biomass: evidence for microbial assimilation of ancient organic carbon during black shale weathering., Science, 292: 1127-1131. [176]

Petsch, S. T., Smernik, R. J., Eglinton, T. I., and Oades, J. M. (2001b), A solid state ${ }^{13} \mathrm{C}-\mathrm{NMR}$ study of kerogen degradation during black shale weathering, Geochimica et Cosmochimica Acta, 65: 1867-1872. [176]

Peucker-Ehrenbrink, B. and Hannigan, R. E. (2000), Effects of black shale weathering on the mobility of rhenium and platinum group elements, Geology, 28: 475-478. $[17,76,78,150,151,176,179,198]$

Peucker-Ehrenbrink, B. and Jahn, B.-m. (2001), Rhenium-osmium isotope system- 
atics and platinum group element concentration: Loess and the upper continental crust, Geochmistry Geophysics Geosystems, 2: doi: 10.1029/2001GC000172. [184]

Pierson-Wickmann, A.-C., Reisberg, L., and France-Lanord, C. (2003), Behavior of Re and Os during low-temperature alteration: results from Himalayan soils and altered black shales during low-temperature alteration: results from Himalayan soils and altered black shales, Geochimica et Cosmochimica Acta, 66: 1539-1548. $[17,76,87,176,179,198]$

Quirke, J. M. E. (1987), Metal Complexes in Fossil Fuels: Geochemistry, Characterization, and Processing, chap. Rationalization for the predominance of vanadium and nickel porphyrins in the geosphere, American Chemical Society, 74-83. [189]

Rayleigh, J. W. S. (1902), On the distillation of binary mixtures, Philosophical Magazine, 4: 521-537. [135, 187]

Siebert, C., Nägler, T. F., von Blanckenburg, F., and Kramers, J. D. (2003), Molybdenum isotope records as a potential new proxy for paleoceanography, Earth and Planetary Science Letters, 211: 159-171. [15, 16, 17, 22, 76, 91, 92, 94, 98, 102, $104,176,179,186,199]$

Sun, W., Bennett, V. C., Eggins, S. M., Kamenetsky, V. S., and Arculus, R. J. (2003), Enhanced mantle-to-crust rhenium transfer in undegassed arc magmas, Nature, 422: 294-297. [73, 176, 184]

Thompson, A., Ruiz, J., A., C. O., Titus, M., and Chorover, J. (2007), Rayleigh fractionation of iron isotopes during pedogenesis along a climate sequence of a Hawaiian basalt, Chemical Geology, 238: 72-83. [188]

Tuttle, M. L. W., Breit, G. N., and Goldhaber, M. B. (2003), Geochemical data from New Albany Shale, Kentucky: a study of metal mobility during weathering of black shales, U. S. Geological Survey Open-file Report, 03-207: 1-57. [176] 
Weyer, S., Anbar, A. D., Gerdes, A., Gordon, G. W., Algeo, T. J., and Boyle, E. A. (2008), Natural fractionation of ${ }^{238} \mathrm{U} /{ }^{235} \mathrm{U}$, Geochimica et Cosmochimica Acta, 72 : 345-359. [16, 17, 176, 199] 


\section{Chapter 5}

\section{Conclusions and future avenues of research}

This study reassesses the surface-cycling of Mo and Re, develops a method for the precise measurement of stable isotope analyses of $\operatorname{Re}\left(\delta^{187} \operatorname{Re}\right)$, and applies $\delta^{187} \operatorname{Re}$ to a modern organic-weathering environment. The results significantly enhance the elemental and now isotopic application of Re to the study of modern and ancient redox processes.

New world river averages of Mo and Re are calculated with a survey of 38 global exorheic rivers representing $37 \%$ of total global $\mathrm{H}_{2} \mathrm{O}$ runoff and $25 \%$ of total exorheic drainage area. Comparison of the obtained world river averages of major cations confirms that the river sample subset is representative of the global river

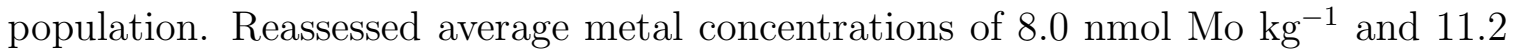
pmol $\mathrm{Re} \mathrm{kg}^{-1}$ (corrected for anthropogenic contribution) are significantly higher than

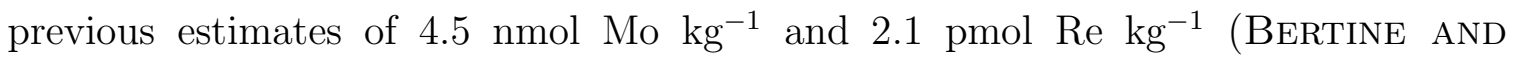
Turekian, 1973; Colodner et AL., 1993). The proportion of the river Re flux that is anthropogenically-sourced can be estimated at $~ 33 \%$ based on the correlation between dissolved $\mathrm{Re}$ and $\mathrm{SO}_{4}^{2-}$ observed across orders of magnitude and a variety of sample substrates. 
Detailed studies of the Hudson River estuary indicate a significant, transient, Re source at, or upstream of, the Federal Dam at Troy, NY. The Re enrichment is not due to estuarine mixing, or sediment resuspension. Given the industrial history of the Hudson River, it is possible that the feature is anthropogenic.

This study reports the first concentration data of Re in hydrothermal fluids. Endmember hydrothermal fluids from the Manus Basin contain essentially no Re and have Mo concentrations significantly lower than ambient seawater. Though hightemperature hydrothermal alteration serves as sink of seawater Mo and Re, the fluxes are negligible compared to the riverine fluxes of these metals seawater.

The 1.8- and 7.9-fold increases in respective Mo and Re (pre-anthropogenic) river concentrations result in corresponding decreases in the oceanic response times of these metals from $8.0 \times 10^{5} \mathrm{yr}\left(\mathrm{Mo}\right.$; MORFORD AND EMERSON, 1999) and $7.5 \times 10^{5}$ yr (Re; Colodner ET AL., 1993) to $4.4 \times 10^{5} \mathrm{yr}(\mathrm{Mo})$ and $1.3 \times 10^{5} \mathrm{yr}$ (Re, preanthropogenic). Molybdenum and especially Re are, therefore, significantly more sensitive to changing source and sink fluxes than was previously thought. These new values of $\tau_{\mathrm{Mo}}$ and $\tau_{\mathrm{Re}}$ must be considered in the interpretation of elemental and isotopic signatures of these metals in the geologic record.

The development of analytical methods for stable Re isotope analyses was precipitated by the existence of established Fe and Mo isotope redox proxies (e.g., Arnold ET AL., 2004; Dauphas ET AL., 2004; AnBar ET AL., 2007) and by the strong response of Re concentrations to changes in redox environment (KOIDE ET AL., 1986; Ravizza et Al., 1991; Crusius et Al., 1996; Crusius and Thomson, 2000; Peucker-Ehrenbrink And Hannigan, 2000; Jaffe et Al., 2002; PiersonWickmann ET AL., 2003). Though hampered by low Re abundances in most materials and the necessity of correcting for isobaric ${ }^{187} \mathrm{Os}, \delta^{187}$ Re values are reproducible at the $0.1 \%$-level (2 s.d.) for analyte concentrations of $10 \mathrm{ng} \mathrm{Re}^{-1}$, and at $0.05 \%$ for concentrations of $20 \mathrm{ng} \mathrm{Re}^{-1}$. Our initial assessment identifies $0.3 \%$ variability in five commercially available Re materials. 
The application of Re isotope analyses to a redox-variable environment observed a $0.5 \%$ range in $\delta^{187}$ Re across a weathering profile of Devonian New Albany Shale, and brings the total documented range of $\delta^{187}$ Re to $0.9 \%$. Highly-weathered (low $\mathrm{C}_{\text {org }}$ ) portions of the outcrop are characterized by low abundances of isotopically light Re, while Re in the unweathered portions is both more abundant and isotopically heavy. A two-component mixing model and a model of Rayleigh fractionation both account for the observed Re fractionation equally well.

Two-component mixing implies the differential, non-fractionating weathering of isotopically distinct Re reservoirs, while a Rayleigh process describes changing $\delta^{187} \operatorname{Re}$ as the progression of a consistently fractionating weathering process on a single metal reservoir. If the observed fractionation is the result of two-component mixing $\delta^{187} \mathrm{Re}$ acts as a tracer of different Re sources; if the Rayleigh model is correct, $\delta^{187}$ Re may serve as an indicator of the extent of $\mathrm{C}_{\text {org }}$ weathering. Though there is insufficient data to rule out either fractionation model, both are amenable to testing.

The combination of the development of Re isotope analysis and observed natural $\delta^{187}$ Re variability with a more dynamic Re seawater reservoir broadens the applicabil-

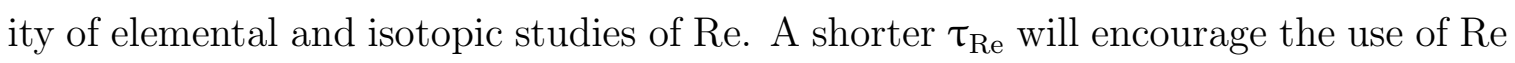
in evaluating changing oceanic metal inventories as has been done for Mo (EMERSON And Huested, 1991; Algeo, 2004), while high Re concentrations in polluted samples may provide a means of monitoring anthropogenic metal contributions to the environment.

Due to their geochemical similarities, Re serves as a natural complement to the well-established Mo and more recently developed U isotope systems (SIEBERT ET AL., 2003; Arnold et Al., 2004; Pearce et Al., 2008; Weyer et Al., 2008). If Re isotopes record the extent of ocean anoxia as Mo isotopes are thought to do, the comparative $\tau_{\mathrm{Re}}$ and $\tau_{\mathrm{Mo}}$ would result in a more dynamic $\delta^{187}$ Re signature relative to $\delta^{98 / 95}$ Mo. Alternatively, Re isotopic composition of $\mathrm{C}_{\text {org }}$-rich sediments may not be sensitive to ocean anoxia as Re is thought to have a very small oxic sink (KOIDE 
ET AL., 1986). Palaeoredox studies using Mo isotope palaeoredox studies have been complicated by the intermediate $\delta^{98 / 95}$ Mo values of suboxic sediments (POULSON ET AL., 2006). While oxic sediments to not appear to sequester Re, suboxic sediments are thought to be a significant sink (MORFORD AND EMERSON, 1999); if suboxic accumulation imparts a measurable $\delta^{187}$ Re fractionation, it might be possible to use $\delta^{187}$ Re to subtract the suboxic component from the $\delta^{98 / 95}$ Mo signature.

Because the Re isotope system is essentially unexplored and the dynamism of Re in seawater is newly recognized, essentially any future studies will yield valuable information. 


\section{Bibliography}

Algeo, T. J. (2004), Can marine anoxic events draw down the trace element inventory of seawater?, Geology, 32: 1057-1060. [15, 91, 92, 175, 199]

Anbar, A. D., Duan, Y., Lyons, T. W., Arnold, G. L., Kendall, B., Creaser, R. A., Kaufman, A. J., Gordon, G. W., Scott, C., Garvin, J., and Buick, R. (2007), A whiff of oxygen before the great oxidation event?, Science, 317: 1903-1906. [198]

Arnold, G. L., Anbar, A. D., Barling, J., and Lyons, T. W. (2004), Molybdenum isotope evidence for widespread anoxia in mid-Proterozoic oceans, Science, 304: 87-90. [16, 22, 98, 104, 129, 175, 176, 186, 198, 199]

Bertine, K. K. and Turekian, K. K. (1973), Molybdenum in marine deposits, Geochimica et Cosmochimica Acta, 37: 1415-1434. [15, 16, 21, 23, 24, 26, 65, 69, 70, 71, $74,76,91,94,95,102,197]$

Colodner, D., Sachs, J., Ravizza, G., Turekian, K., Edmond, J., and Boyle, E. (1993), The geochemical cycle of rhenium: a reconnaissance, Earth and Planetary Science Letters, 117: 205-221. [15, 16, 21, 22, 23, 24, 26, 65, 69, 70, 71, 76, 79, 86, 87, 91, $92,94,95,96,102,150,151,197,198]$

Crusius, J., Calvert, S., Pederson, T., and Sage, D. (1996), Rhenium and molybdenum enrichments in sediments as indicators of oxic, suboxic and sulfidic conditions of deposition, Earth and Planetary Science Letters, 145: 65-78. [15, 17, 21, 22, 75, $92,176,186,198]$

Crusius, J. and Thomson, J. (2000), Comparative behavior of authigenic Re, U, and Mo during reoxidation and subsequent long-term burial in marine sediments, Geochimica et Cosmochimica Acta, 64: 2233-2242. [176, 198]

Dauphas, N., van Zuilen, M., Wadhwa, M., Davis, A. M., Marty, B., and Janney, P. 
(2004), Clues from Fe isotope variations on the origin of early Archean BIFs from Greenland, Science, .06: 2077-2080. [198]

Emerson, S. R. and Huested, S. S. (1991), Ocean anoxia and the concentrations of molybdenum and vanadium in seawater, Marine Chemistry, 34: 177-196. [15, 92, 199]

Jaffe, L. A., Peucker-Ehrenbrink, B., and Petsch, S. T. (2002), Mobility of rhenium, platinum group elements and organic carbon during black shale weathering, Earth and Planetary Science Letters, 198: 339-353. [17, 21, 76, 176, 177, 178, 179, 181, $183,186,198]$

Koide, M., Hodge, V. F., Yang, J. S., Stallard, M., Goldberg, E. G., Calhoun, J., and Bertine, K. K. (1986), Some comparative marine chemistries of rhenium, gold, silver and molybdenum, Applied Geochemistry, 1: 705-714. [17, 75, 76, 88, 176, $179,198,199]$

Morford, J. L. and Emerson, S. (1999), The geochemistry of redox sensitive trace metals in sediments, Geochimica et Cosmochimica Acta, 63: 1735-1750. [15, 16, $17,21,22,23,75,91,92,96,175,198,200]$

Pearce, C. R., Cohen, A. S., Coe, A. L., and Burton, K. W. (2008), Molybdenum isotope evidence for global ocean anoxia coupled with perturbations to the carbon cycle during the Early Jurassic, Geology, 36: 231-234. [16, 93, 98, 175, 199]

Peucker-Ehrenbrink, B. and Hannigan, R. E. (2000), Effects of black shale weathering on the mobility of rhenium and platinum group elements, Geology, 28: 475-478. $[17,76,78,150,151,176,179,198]$

Pierson-Wickmann, A.-C., Reisberg, L., and France-Lanord, C. (2003), Behavior of Re and Os during low-temperature alteration: results from Himalayan soils and altered black shales during low-temperature alteration: results from Himalayan 
soils and altered black shales, Geochimica et Cosmochimica Acta, 66: 1539-1548. $[17,76,87,176,179,198]$

Poulson, R. L., Siebert, C., McManus, J., and Berelson, W. M. (2006), Authigenic molybdenum isotope signatures in marine sediments, Geology, 34: 617-620. [16, $22,91,200]$

Ravizza, G. E., Turekian, K. K., and Hay, B. J. (1991), The geochemistry of rhenium and osmium in recent sediments from the Black Sea, Geochimica et Cosmochimica Acta, 55: 3741-3752. [15, 75, 150, 151, 198]

Siebert, C., Nägler, T. F., von Blanckenburg, F., and Kramers, J. D. (2003), Molybdenum isotope records as a potential new proxy for paleoceanography, Earth and Planetary Science Letters, 211: 159-171. [15, 16, 17, 22, 76, 91, 92, 94, 98, 102, 104, 176, 179, 186, 199]

Weyer, S., Anbar, A. D., Gerdes, A., Gordon, G. W., Algeo, T. J., and Boyle, E. A. (2008), Natural fractionation of ${ }^{238} \mathrm{U} /{ }^{235} \mathrm{U}$, Geochimica et Cosmochimica Acta, 72: 345-359. [16, 17, 176, 199] 